\title{
Aerodynamic drag reduction of a racing motorcycle through vortex generation
}

\author{
Gerald M. Angle II \\ West Virginia University
}

Follow this and additional works at: https://researchrepository.wvu.edu/etd

\section{Recommended Citation}

Angle, Gerald M. II, "Aerodynamic drag reduction of a racing motorcycle through vortex generation" (2002). Graduate Theses, Dissertations, and Problem Reports. 1274.

https://researchrepository.wvu.edu/etd/1274

This Thesis is protected by copyright and/or related rights. It has been brought to you by the The Research Repository @ WVU with permission from the rights-holder(s). You are free to use this Thesis in any way that is permitted by the copyright and related rights legislation that applies to your use. For other uses you must obtain permission from the rights-holder(s) directly, unless additional rights are indicated by a Creative Commons license in the record and/ or on the work itself. This Thesis has been accepted for inclusion in WVU Graduate Theses, Dissertations, and Problem Reports collection by an authorized administrator of The Research Repository @ WVU. For more information, please contact researchrepository@mail.wvu.edu. 


\title{
Aerodynamic Drag Reduction of a Racing Motorcycle through Vortex Generation
}

By

Gerald M. Angle II

\author{
A Thesis Submitted to the \\ College of Engineering and Mineral Resources \\ At West Virginia University \\ In Partial Fulfillment of the Requirements \\ for the Degree of \\ Master of Science
}

Department: Mechanical and Aerospace Engineering Major: Aerospace Engineering

\author{
West Virginia University \\ Morgantown, WV \\ 2002
}

Keywords: Drag Reduction, Motorcycle, Vortex Generators 


\section{ABSTRACT \\ Aerodynamic Drag Reduction of a Racing Motorcycle through Vortex Generation \\ Gerald M Angle II}

Interest has been expressed in reducing the aerodynamic drag of a racing-class motorcycle. The drag can be reduced through either an overall redesign of the exterior shape or through some type of flow control mechanism. Due to the limitations imposed on the length of the motorcycle set by race officials, and due to the constraints of the racing circuit, major changes to the shape of the fairings are not practical. Thus a more practical choice for drag reduction is to use flow control techniques aimed at reducing the size of the wake of the motorcycle.

There are several different types of flow control devices including vortex generation, suction or a blowing jet. The use of either blowing jets or suction is not desirable because of the required power to perform these tasks. On the other hand, using vortex generation required no additional power and, if aligned properly, can noticeably reduce the drag. Multiple vortex generating devices exist, that range from strategically located metal vanes that induce small vortices to a dimple tape that introduces turbulence into the boundary layer.

For this research it was determined that the metal vortex generating vanes would be used as the flow control device. Testing was conducted in multiple phases between the WVU Closed Loop Wind Tunnel (WVUCLT) and the Langley Full Scale Tunnel (LFST). All of the testing was conducted with no tire rotation, on a stationary ground plane with the model elevated out of the boundary layer of the ground.

Despite the effects of tunnel blockage on the vortex generator effectiveness, it was found that vortex generators could effectively reduce the drag. Phase I of testing resulted in a maximum drag reduction of 118 drag counts (10.1\%) from the baseline, or an increase of up to 355 drag counts (30.5\%), depending on the geometric configuration. The results from Phase II showed no significant reduction or increase in the drag coefficient. They produced a reduction of $48 \mathrm{drag}$ counts (7.8\%) and an increase of 217 drag counts $(35.2 \%)$ for Phase II tests. Therefore, it is possible to use vortex generators to reduce the drag on a motorcycle. However, the configuration of these vortex generators that can provide the maximum drag reduction in a real scenario (full scale Reynolds numbers; with tire rotation and moving ground plane) is not known at this time. 


\section{Table of Contents}

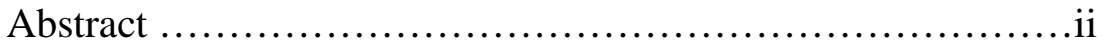

List of Figures......................................

List of Tables ..........................................xii

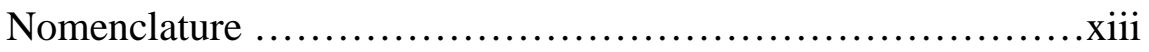

Acknowledgements.........................................

Chapter 1.0 Introduction..................................... 1

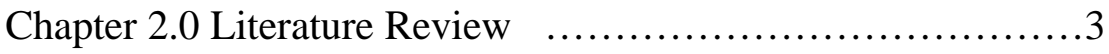

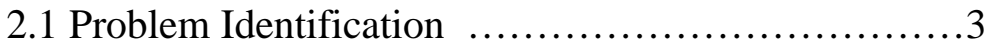

2.2 General Drag Reduction ......................4

2.3 Tire Rotation and Ground Simulation $\ldots . \ldots \ldots \ldots . . .5$

2.4 Wind Tunnel Blockage ..........................13

2.5 Turbulence Control and Velocity Profile .............15

2.6 Vortex Generators ................................ 18

Chapter 3.0 Experimental Apparatus ........................24

3.1 Tul-Aris Model and Vortex Generators...............24

3.2 Testing Apparatus used at West Virginia University...29

3.3 Testing Apparatus used at Old Dominion University...37

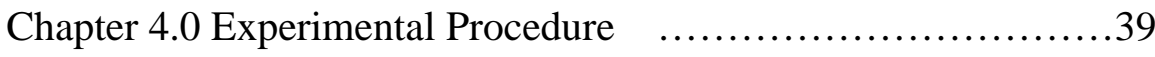

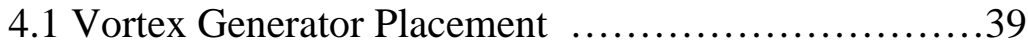

4.2 Preliminary Testing..............................40

4.3 Test Matrix $\ldots \ldots \ldots \ldots \ldots \ldots \ldots \ldots \ldots \ldots \ldots \ldots \ldots \ldots . \ldots, \ldots \ldots \ldots$

4.4 Wind Tunnel Testing Procedure......................46 


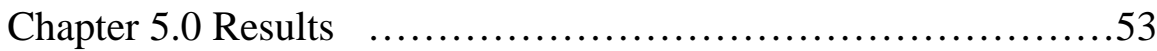

5.1 Phase I Results................................53

5.2 Phase II Results ..............................6 63

5.3 Phase III Results ................................74

5.4 Summary and Conclusions .......................79

Chapter 6.0 Conclusions and Recommendations ................82

6.1 Conclusions...................................82

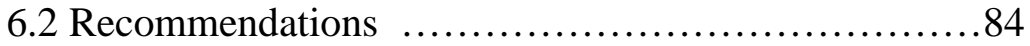

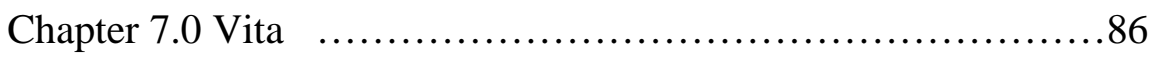

Chapter 8.0 References...................................87

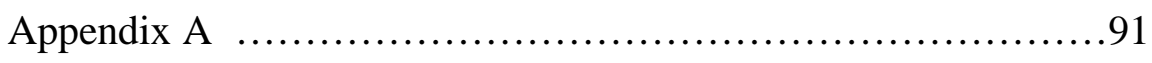

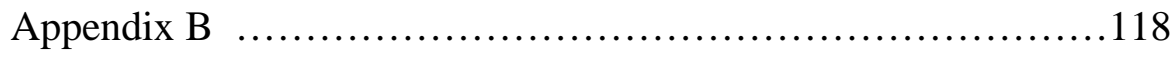

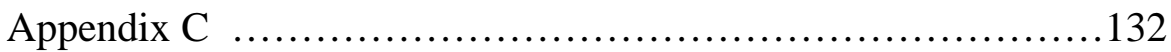




\section{List of Figures}

Figure 2.1: Flow Model for the Vortex Flow and Rolling Wheel. .................6

Figure 2.2: Three Zones of Automotive Underbody Airflow. .....................8

Figure 2.3: Horizontal and Vertical Velocity Profiles Without Damping Screen. . ..17

Figure 2.4: Horizontal and Vertical Velocity Profiles With Damping Screen. ........18

Figure 2.5: Vortex Generator Effects of Airflow over 2-D Airfoil. ..................19

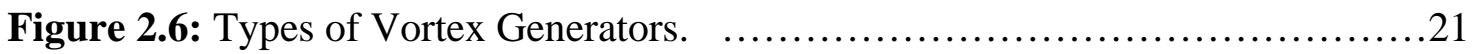

Figure 3.1: Side View of Tul-Aris Model, Without 'Dummy' Rider. ...............25

Figure 3.2: View inside Tul-Aris Model, Without Rider. .........................26

Figure 3.3: Picture of Pressure Tap Locations on the Lower Fairing of the Model. ...27

Figure 3.4: $1 / 2$-inch Vortex Generator provided by Mr. Gary Wheeler. .............27 A. Front View B. Side View

Figure 3.5: Modified 1/4-inch Vortex Generator. 28 A. Front View B. Side View

Figure 3.6: Modified 1/8-inch Vortex Generator 28 A. Top View B. Side View

Figure 3.7: Schematic of WVU Closed Loop Wind Tunnel With Locations of Flow Straightening Screens and Diffuser. ..........................29

Figure 3.8: Comparison of Non-Dimensional Horizontal Velocity Profiles With No Flow Straightening Screen.

Figure 3.9: Comparison of Non-Dimensional Horizontal Velocity Profiles With Flow Straightening Screen. ........................................ 31

Figure 3.10: Frontal View of Tul-Aris Model for Frontal Area Determination. ….33

Figure 3.11: Calibration Curve for the Omegadyne Load Cell. .......................35

Figure 3.12: Sketch of Device used to Calibrate Scani-Valve. ......................35

Figure 3.13: Calibration Curve for the Scani-Valve Pressure Transducer. . ...........36 
Figure 3.14: Photograph of Pitot-Static Tube installed along the Side of the Model near the Location of Maximum Thickness During the Increased Blockage Test. ................................................. 37

Figure 3.15: Photograph of the Tul-Aris Model and Sting Installed in the Langley

Full Scale Tunnel.

Figure 4.1: Initial Shape of the Upper Fairing of the Tul-Aris Motorcycle. .40

Figure 4.2: Final Shape of the Upper Fairing after Preliminary Testing.

Figure 5.1: Comparison of Relative Drag Coefficients for Phase I of Testing Conducted in the West Virginia University Closed Loop Wind Tunnel. .57

Figure 5.2: Effects of Vortex Generator Height on Configuration 1. 57

Figure 5.3: Effects of Vortex Generator Height on Configuration 2. ...............58

Figure 5.4: Effects of Vortex Generator Height on Configuration 3. ..............58

Figure 5.5: Effects of Vortex Generator Height on Configuration 4. ...............59

Figure 5.6: Effects of Vortex Generator Height on Configuration 5. ..............59

Figure 5.7: Phase I Average Pressure Coefficients for Three Baseline 1 Tests. .....60

Figure 5.8: Phase I Average Pressure Coefficients for Three 1/2-inch VG

Configuration 1 Tests.

Figure 5.9: Phase I Average Pressure Coefficients for Three 1/4-inch VG Configuration 2 Tests.

Figure 5.10: Phase I Average Pressure Coefficients for Three $1 / 8$-inch VG

Configuration 3 Tests. 61

Figure 5.11: Phase I Average Pressure Coefficients for 1/8-inch VG Modified

Configuration 1 Test.

Figure 5.12: Comparison of Phase I Average Pressure Coefficients for Pressure Tap \#1.

Figure 5.13: Comparison of Phase I Average Pressure Coefficients for Pressure Tap \#23. 
Figure 5.14: Comparison of Relative Drag Coefficients for Phase II of Testing,

Conducted in the Langley Full Scale Wind Tunnel.

Figure 5.15: Variation in Drag Coefficient with Free stream Velocity for the

Phase II Baseline Configuration.

Figure 5.16: Variation in Drag Coefficient with Free stream Velocity for the Phase II 1/2 inch Vortex Generator Configurations 4 and 5.

Figure 5.17: Variation in Drag Coefficient with Free stream Velocity for the Phase II 1/4 inch Vortex Generator Configurations 4 and 5.

Figure 5.18: Variation in Drag Coefficient with Free stream Velocity for the Phase II 1/8 inch Vortex Generator Configurations 4 and 5.

Figure 5.19: Average Pressure Coefficients for Phase II Run 4.02. .70

Figure 5.20: Average Pressure Coefficients for Phase II Run 5.02. .70

Figure 5.21: Average Pressure Coefficients for Phase II Run 6.03. .71

Figure 5.22: Average Pressure Coefficients for Phase II Run 7.03. .71

Figure 5.23: Average Pressure Coefficients for Phase II Run 8.01. .72

Figure 5.24: Average Pressure Coefficients for Phase II Run 9.01. .72

Figure 5.25: Average Pressure Coefficients for Phase II Run $10.01 \ldots \ldots \ldots \ldots \ldots \ldots . \ldots 73$

Figure 5.26: Comparison of Phase II Average Pressure Coefficients for Pressure Tap \#5. .73

Figure 5.27: Comparison of Phase II Average Pressure Coefficients for Pressure

Tap \#26.

Figure 5.28: Comparison of Relative Drag Coefficients for Phase III of Testing Conducted in the West Virginia University Closed Loop Wind Tunnel....

Figure 5.29: Upper Fairing Parametric Plot of Drag Coefficient as Dependent of Vortex Generator Location from the Leading Edge.

Figure 5.30: Lower Fairing Parametric Plot of Drag Coefficient as Dependent of Vortex Generator Location from the Leading Edge.

Figure 5.31: Effects of Test Section Blockage on the Drag Coefficient. 78 
Figure A.1: Phase I Average Pressure Coefficients for Three Baseline 2 Tests. .....92

Figure A.2: Phase I Average Pressure Coefficients for Three Baseline 3 Tests. .....92

Figure A.3: Phase I Average Pressure Coefficients for Three Baseline 4 Tests. . ..93

Figure A.4: Phase I Average Pressure Coefficients for Modified Baseline Test. ...93

Figure A.5: Phase I Average Pressure Coefficients for Three 1/2-inch VG

Configuration 2 Tests. .....................................94

Figure A.6: Phase I Average Pressure Coefficients for Three 1/2-inch VG

Configuration 3 Tests. ....................................94

Figure A.7: Phase I Average Pressure Coefficients for Three 1/2-inch VG

Configuration 4 Tests. ..........................................95

Figure A.8: Phase I Average Pressure Coefficients for Three 1/2-inch VG

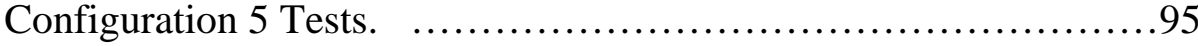

Figure A.9: Phase I Average Pressure Coefficients for Three 1/4-inch VG

Configuration 1 Tests. ....................................96

Figure A.10: Phase I Average Pressure Coefficients for Three 1/4-inch VG

Configuration 3 Tests. ........................................96

Figure A.11: Phase I Average Pressure Coefficients for Three 1/4-inch VG

Configuration 4 Tests. .....................................97

Figure A.12: Phase I Average Pressure Coefficients for Three 1/4-inch VG Configuration 5 Tests.

Figure A.13: Phase I Average Pressure Coefficients for Three 1/8-inch VG

Configuration 1 Tests.

Figure A.14: Phase I Average Pressure Coefficients for Three 1/8-inch VG Configuration 2 Tests.

Figure A.15: Phase I Average Pressure Coefficients for Three 1/8-inch VG Configuration 4 Tests.

Figure A.16: Phase I Average Pressure Coefficients for Three 1/8-inch VG Configuration 5 Tests. 
Figure A.17: Phase I Average Pressure Coefficients for 1/8-inch VG Modified Configuration 2 Test. 100

Figure A.18: Phase I Average Pressure Coefficients for 1/8-inch VG Modified Configuration 3 Test.

Figure A.19: Phase I Average Pressure Coefficients for 1/8-inch VG Modified Configuration 4 Test. 101

Figure A.20: Phase I Average Pressure Coefficients for 1/8-inch VG Modified Configuration 5 Test.

Figure A.21: Comparison of Phase I Average Pressure Coefficients for Pressure Tap \#5. 102

Figure A.22: Comparison of Phase I Average Pressure Coefficients for Pressure Tap \#6. 102

Figure A.23: Comparison of Phase I Average Pressure Coefficients for Pressure Tap \#24. 103

Figure A.24: Comparison of Phase I Average Pressure Coefficients for Pressure Tap \#25. 103

Figure A.25: Comparison of Phase I Average Pressure Coefficients for Pressure Tap \#26. 104

Figure A.26: Comparison of Phase I Average Pressure Coefficients for Pressure Tap \#27. 104

Figure A.27: Comparison of Phase I Average Pressure Coefficients for Pressure Tap \#28. 105

Figure A.28: Comparison of Phase I Average Pressure Coefficients for Pressure Tap \#29. 105

Figure A.29: Comparison of Phase I Average Pressure Coefficients for Pressure Tap \#30. 106

Figure A.30: Average Pressure Coefficients for Phase II Run 4.01. .106

Figure A.31: Average Pressure Coefficients for Phase II Run 4.03. 107

Figure A.32: Average Pressure Coefficients for Phase II Run 5.01. .107

Figure A.33: Average Pressure Coefficients for Phase II Run 5.03. 108 
Figure A.34: Average Pressure Coefficients for Phase II Run 6.01. 108

Figure A.35: Average Pressure Coefficients for Phase II Run 6.02. 109

Figure A.36: Average Pressure Coefficients for Phase II Run 7.01. 109

Figure A.37: Average Pressure Coefficients for Phase II Run 7.02. 110

Figure A.38: Average Pressure Coefficients for Phase II Run 8.02. 110

Figure A.39: Average Pressure Coefficients for Phase II Run 8.03. 111

Figure A.40: Average Pressure Coefficients for Phase II Run 8.04.

Figure A.41: Average Pressure Coefficients for Phase II Run 8.05. 112

Figure A.42: Average Pressure Coefficients for Phase II Run 8.06.

Figure A.43: Comparison of Phase II Average Pressure Coefficients for Pressure Tap \#1. 113

Figure A.44: Comparison of Phase II Average Pressure Coefficients for Pressure Tap \#6.

Figure A.45: Comparison of Phase II Average Pressure Coefficients for Pressure Tap \#23. 114

Figure A.46: Comparison of Phase II Average Pressure Coefficients for Pressure Tap \#24. 114

Figure A.47: Comparison of Phase II Average Pressure Coefficients for Pressure Tap \#25.

Figure A.48: Comparison of Phase II Average Pressure Coefficients for Pressure Tap \#27. 115

Figure A.49: Comparison of Phase II Average Pressure Coefficients for Pressure Tap \#28. 116

Figure A.50: Comparison of Phase II Average Pressure Coefficients for Pressure Tap \#29. 116

Figure A.51: Comparison of Phase II Average Pressure Coefficients for Pressure Tap \#30. 
Figure B.1: LabView Program used for Load Cell Calibration.
A. Step 1 of Program
B. Step 2 of Program

Figure B.2: Step 1 of LabView Program used for Scani-Valve Calibration. 120

Figure B.3: Step 2 of LabView Program used for Scani-Valve Calibration. 121

Figure B.4: Step 1 of LabView Code used for Data Acquisition. 122

Figure B.5: Step 2 of LabView Code used for Data Acquisition. 123

Figure B.6: Step 3 of LabView Code used for Data Acquisition. 124

Figure B.7: Step 4 of LabView Code used for Data Acquisition. 125

Figure B.8: Step 5 of LabView Code used for Data Acquisition. 126

Figure B.9: Step 6 of LabView Code used for Data Acquisition. 127

Figure B.10: Step 7 of LabView Code used for Data Acquisition. 128

Figure B.11: Step 8 of LabView Code used for Data Acquisition. 129

Figure B.12: Step 9 of LabView Code used for Data Acquisition.

Figure B.13: LabView Code used for Data Acquisition Measuring Drag and Temperature. 


\section{List of Tables}

Table 2.1: Summary of Test Results from Beauvais, et $\mathrm{al}^{3}$. $\quad$....................13

Table 2.2: Experimental and CFD Strengths of Vortices as Determined by

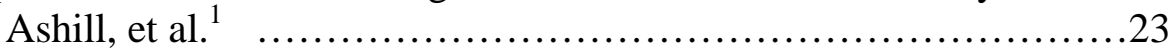

Table 4.1: Test Matrix for Tul-Aris Wind Tunnel Testing at WVU, Phase I. .....43

Table 4.2: Test Matrix for Phase II of Testing in the Langley Full Scale Wind Tunnel. .....................................................44

Table 4.3: Test Matrix for Phase III of Tests, Conducted at WVU. ................45

Table 4.4: Instrumentation Errors for Measuring Devices. .....................52

Table 5.1: Measured Change in Drag Coefficient and the Standard Deviation of the Measurement for Phase I of Testing.........................54

Table 5.2: Drag Coefficients of the $55 \mathrm{ft} / \mathrm{s}$ tests Conducted in the LFST, Phase II.

Table 5.3: Drag Coefficients of the $70 \mathrm{ft} / \mathrm{s}$ tests Conducted in the LFST, Phase II.

Table 5.4: Drag Coefficients of the $120 \mathrm{ft} / \mathrm{s}$ tests Conducted in the LFST, Phase II. 64

Table 5.5: Drag Coefficients for the New Configurations Tested in the LFST. ...66

Table 5.6: Measured Change in Drag Coefficient and the Corresponding Standard Deviation for the Given Number of Tests Conducted During Phase III. ........................................... 76

Table 5.7: Effect of Blockage on Drag Coefficient and the Associated Test

Section Area. ...............................................76

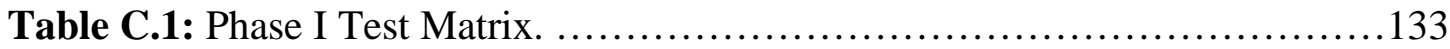

Table C.2: Phase I Test Matrix, Continued. ...................................134

Table C.3: Phase III Test Matrix. ..........................................135

Table C.4: Phase III Test Matrix. ................................................136

Table C.5: Phase III Test Matrix. .................................................137

Table C.6: Phase III Test Matrix. ............................................138 


\section{Nomenclature}
$\mathrm{A}, \mathrm{A}_{\mathrm{fr}}$
Model frontal area $\left(\mathrm{in}^{2}, \mathrm{ft}^{2}\right)$
BR
Blockage ratio
c
Chord length (in, ft)
$\mathrm{C}_{\mathrm{D}}$
Coefficient of Drag
$\mathrm{C}_{\mathrm{L}}$
Coefficient of Lift
$\mathrm{C}_{\mathrm{p}}$
Coefficient of Pressure
$\mathrm{C}_{\mathrm{Q}}$
Suction Parameter
D
Diameter; Drag force (in, $\mathrm{ft}$ ); (lbs)
Damping factor
g
Gravitational constant $\left(\mathrm{ft} / \mathrm{s}^{2}\right)$
$\mathrm{h}$
Span; Device height; Manometer height (in, ft); (in); (in $\mathrm{H}_{2} \mathrm{O}$ )
$\mathrm{h}_{\mathrm{L}}$
Head losses in WVUCLT (in $\mathrm{H}_{2} \mathrm{O}$ )
K
Pressure drop coefficient
$\mathrm{L}$
Length (in, ft)
LFST
Langley Full Scale Tunnel
MVG
Micro-Vortex Generators
$\mathrm{P}$
Pressure (psi, psf)
$\mathrm{R}$
Cross plane distance to center of vortex; Universal gas constant; Result (in, ft); (ft-lb/slug- $\left.{ }^{\circ} \mathrm{R}\right)$
$\mathrm{R}^{\prime} \quad$ Gas constant $\left(\mathrm{ft}-\mathrm{lb} /\right.$ slug- $\left.^{\mathrm{O}} \mathrm{R}\right)$
Re Reynolds number
S
Slot width (in, $\mathrm{ft}$ )
Test section cross-sectional area $\left(\mathrm{in}^{2}, \mathrm{ft}^{2}\right)$

f 


$\begin{array}{ll}\text { SBVG } & \text { Sub-Boundary Layer Vortex Generator } \\ \text { STDEV } & \text { Standard deviation } \\ \mathrm{t} & \text { Time }(\mathrm{sec}) \\ \mathrm{T} & \text { Temperature }\left({ }^{\circ} \mathrm{F},{ }^{\circ} \mathrm{R}\right) \\ \mathrm{U} & \text { Velocity }(\mathrm{ft} / \mathrm{s}, \mathrm{mph}) \\ U_{\infty} & \text { Free stream velocity }(\mathrm{ft} / \mathrm{s}, \mathrm{mph}) \\ \mathrm{V} & \text { Velocity (ft/s, mph) } \\ \mathrm{VG} & \text { Vortex Generator } \\ \mathrm{W} & \text { Width; Uncertainty (in, ft) } \\ \mathrm{WVUCLT} & \text { West Virginia University Closed Loop Wind Tunnel } \\ \mathrm{x} & \text { Distance along Tunnel } ; \text { Independent Variable (in, ft) } \\ \mathrm{X} & \text { Recorded Signal (V, mV) } \\ \bar{X} & \text { Mean Signal (V, mV) }\end{array}$

\section{Symbols}

$\begin{array}{ll}\alpha & \text { Angle of attack (rad, deg) } \\ \delta & \text { Boundary layer height (in, ft) } \\ \delta_{l x=0} & \text { Boundary layer thickness removed by suction (in, ft) } \\ \Delta \mathrm{C}_{\mathrm{D}} & \text { Change in drag coefficient } \\ \varepsilon_{t}, & \text { Blockage correction factor } \\ \gamma & \text { Specific weight } \\ \Gamma & \text { Circulation } \\ v_{\mathrm{s}} & \text { Suction Velocity }\end{array}$




\begin{tabular}{ll}
$\rho$ & Density \\
$\omega$ & Vorticity \\
$\%$ Diff & Percent difference \\
Subscripts & \\
1 & WVUCLT Small Test Section \\
2 & WVUCLT Large Test Section \\
act & actual... \\
air & ...for air \\
atm & Atmospheric condition \\
cable & ...on the cable \\
D & Drag \\
f & ...on the flat plate \\
fr & Model frontal... \\
L & ...based on length \\
liquid & ...for liquid \\
meas & Measured quantity \\
min & Minimum quantity \\
o & Initial \\
sting & ...on the sting \\
\hline & Test section \\
\hline & Free stream conditions \\
\hline &
\end{tabular}




\section{Acknowledgements}

The WV Space Grant Consortium and the WVU Senate sponsored this work. The author would express his appreciation to Professors John Kuhlman and Gary Morris for their guidance and support in completing this project. A special thanks is also given to the Professor Wade Huebsch for directly supervising this project.

The author would like to thank Mr. Gary Wheeler, Dr. Robin Tuluie and Professor Gary Winn for their contributions towards the completion of this research. Additional thanks are also given to Mr. Brian Hall and Dr. Drew Landman for their assistance in testing in the Langley Full Scale Wind Tunnel and Mr. Jon Henry for his assistance in the WVU Closed Loop Wind Tunnel testing.

Lastly, the author would like to express his appreciation for the encouragement and support provided by his family. 


\subsection{Introduction}

Interest has been expressed in reducing the aerodynamic drag of a racing motorcycle. Drag can be reduced through either an overall redesign of the exterior shape or through some type of flow control mechanism. Due to the limitations on the length of the motorcycle and the demands of the racing circuit, major changes to the shape of the fairings are not practical. Thus it is desired to use a flow control technique to reduce the drag by reducing the size of the wake of the motorcycle.

There are several different types of flow control devices including vortex generation, suction or a blowing jet. The use of either blowing jets or suction is not desirable because of the required power to perform these tasks. This power would either be taken straight from the motor, reducing the power provided to the wheel, or from a smaller additional motor adding to the complexity and overall weight of the motorcycle. Both of these options decrease the optimum performance of the motorcycle. On the other hand, using vortex generation requires no direct power from the motor. However, the use of vortex generators adds a small device drag, but they also produce a reduction in wake (pressure) drag resulting in a net reduction in total drag. Multiple vortex generating devices exist ranging from strategically located metal vanes that induce small vortices to a dimple tape that introduces turbulence into the boundary layer.

For this research it was determined that metal vortex-generating vanes would be used as the flow control device. The model was tufted to visualize the airflow over the fairings, which was used to determine placement of the vortex generators (VG's). Testing was conducted in three phases at two different facilities: the WVU Closed Loop Wind Tunnel (WVUCLT) and the Langley Full Scale Tunnel (LFST). All of the testing 
was conducted with no tire rotation and a stationary ground plane with the model elevated out of the boundary layer of the ground.

The relative drag differences from the baseline (no vortex generators applied to model) configuration were used to determine the effects of the device on the airflow. Two blockage corrections were used to compare the LFST tests to the WVUCLT tests, these were correcting the velocity to the reduced area and the Barlow, Rae and Pope relationship for blocked flow. The effects of vortex generator height, test-section blockage, air-speed were studied. The vortex generator location was parametrically studied on the upper and lower fairings of the motorcycle with respect to the distance from the leading edge. 


\subsection{Literature Review}

This chapter provides information on previous research related to this project. This previous work have primarily focused on automobile testing, most of which can be related to motorcycles as well. The effects of ground simulation, tire rotation and wind tunnel blockage are present on any ground vehicle thus should be addressed when testing a ground vehicle.

\subsection{Problem Identification}

Racing Motorcycles are restricted in the size of the engine by race officials, which limit the top speed of the motorcycle. The output of the engines used in motorcycles are nearing their maximum potential. Therefore, to remain competitive, racing teams will need to look at improving other areas of the design such as the aerodynamics of the motorcycle. One way to reduce the aerodynamic drag would be to reduce the size of the wake created by the motorcycle. The use of vortex generators has been a standard practice in separation control on aircraft wings. However, they can also be used to delay separation on ground vehicles as well. By delaying the separation on the ground vehicle the size of the wake created by the vehicle can be reduced, thus reducing the overall drag. Therefore, application of vortex generators in the proper locations can reduce the overall drag on a motorcycle. By reducing the drag, increases in top speed and fuel economy are achievable.

Wind tunnels can be used to measure the aerodynamic drag and the down-force on any road vehicle. However, to measure the forces and moments it is important to reproduce the actual airflow around the vehicle (Hucho, 1998). Thus the effects of the 
wind tunnel walls, floor, and ceiling need to be addressed. Most available literature addresses these issues for cars, and other large motor vehicles, but little information is available on the wind tunnel effects of motorcycle testing. Due to the opportunity of using an existing full-scale model of the Tul-Aris Motorcycle in the WVU Closed Loop Wind Tunnel, the effects of wind tunnel blockage also need to be addressed and accounted for in experimentation. This project will show that strategically located vortex generators can be added to a motorcycle resulting in a reduction in the total drag of the vehicle.

\subsection{General Drag Reduction}

In general, the drag experienced by a ground vehicle is dependent on the exterior shape of the vehicle and the cross sectional area of the vehicle. According to Hucho (1998) car-like basic bodies of very low drag $\left(C_{D}=0.15\right)$ have been developed and used in some concept car designs. But, these low drag research bodies have not been implemented into production cars, which have a drag coefficient of around 0.30. Starting from a free-flying body, low drag bodies have three factors that increase the drag; the effects of camber, thickness and for truncated bodies, an increase in base pressure.

The lower limit of the vehicle-like basic bodies is in the range of 0.07 to 0.09 , according to Hucho. However, when adding the wheels the drag coefficient increases to 0.14 to 0.16 . Therefore to further reduce the drag the vehicle drag the airflow underneath the car and around the wheels needs to be improved. Another method of reducing the drag is using a higher length to height ratio. Increasing the length is not always the best solution for a production vehicle due to desires of the customer. 
Less conventional methods of reducing the drag on a ground vehicle include base bleed, re-energizing the wake of the vehicle and to reduce the effective base area. The base bleed idea channels the cooling air to the rear of the vehicle where it is then let out into the wake region of the vehicle, increasing the base pressure. The cross section of the base of the vehicle can be reduced through the use of the Coanda effect. This effect is generated when high-speed air is ejected at the lower and upper edges of the rear of the vehicle, which allows the flow to remain attached longer, increasing the base pressure. The wake of the ground vehicle can also be reduced through the use of spoilers, which deflect the airflow toward the centerline of the wake region. This effect may also be achieved with properly placed vortices.

\subsection{Tire Rotation and Ground Simulation}

Two questions that need to be addressed in any ground vehicle testing are simulating the ground beneath the vehicle and whether or not the tires need to be rotating. There have been arguments made for different types of ground simulation as well as whether or not to rotate the tires of the ground vehicle. The impact of simulating the ground effects is, in part, dependent on the type of road vehicle being investigated.

Various opinions exist for the proper way to simulate the ground effects on the moving vehicle. For open wheel vehicles ground simulation is of greater importance than for closed wheel vehicles. Thus wind tunnel experiments need to provide proper ground simulation, as well as wheel rotation for open wheeled vehicles (Barlow, Rae and Pope, 1999). A stationary wheel produces a larger wake region, and an increased vorticity, $\Gamma$,

near the ground than the rotating wheel, see Figure 2.1. Carr $(1970,1994)$ felt that the 
moving ground surface does not significantly improve the simulation, if a gap is present between the wheel and the ground plane. Carr believed that an adequate simulation could be achieved with a small gap between a stationary wheel and a stationary ground plane. Using a gap greater than the boundary layer height avoids the momentum deficit of the boundary layer from the wind tunnel floor.

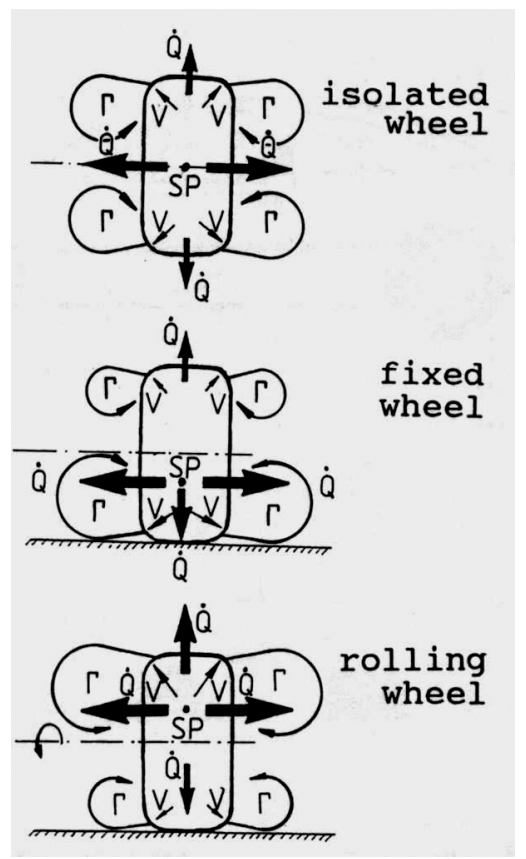

Figure 2.1: Flow Model for the Vortex Flow and Rolling Wheel.

Mercker, et al. (1991), experimentally found that rolling wheels have a large aerodynamic influence on passenger cars. Hackett, et al. (1987) revealed that rolling wheels have a large influence on drag and a limited effect on lift for passenger cars. Passenger cars are generally closed wheel ground vehicles thus the effect on open wheel vehicles is still questionable. Cogotti (1983), testing for the difference between rotating and non-rotating wheels found that a difference of 5 drag counts $\left(\Delta C_{d}=5 / 1000\right)$ was present between the two cases. In a similar experiment Mercker and Knape ${ }^{16}$ found a 
drag coefficient decrease of 27 drag counts when the wheels were rotating as compared to non-rotating wheels. The discrepancy in these two test results is due to differences in the models tested, Cogotti tested an isolated wheel while Mercker and Knape (1989) tested with the wheels attached to a passenger vehicle. It is believed that the motorcycle wheel will appear more like that in Cogotti's testing. However, the difference in drag is expected to be slightly higher than Cogotti's, but a lower drag change than in Mercker and Knape's testing. According to Carr (1994), wheel rotation on an open-wheeled racing-car model generates higher down force on the body and significantly greater body drag.

The simulation of the stationary ground under a moving vehicle can be described in three zones; boundary layer flow, transitional flow, and fully developed flow. Mercker and Wiedemann (1990) describe these zones in detail and these zones are illustrated in Figure 2.2. Each of these three zones of the airflow has different simulation requirements. For Zone 1, boundary layer flow, the bias of a moving ground plane is restricted to the immediate proximity of the wall boundary layer, which is a first-order approximation. But the interaction of the boundary layer and the free stream flow is a second-order effect in the higher order boundary layer theory. Thus both of these approximations need to be addressed in an adequate simulation of the upstream boundary layer flow. In the transitional flow regime, boundary layers are developing from the ground and the underside of the vehicle. In this Zone of the simulation, the viscous effects at any location in the gap between the ground plane and the underside of the vehicle become first-order effects. In Zone 3, the flow will tend to form a velocity profile 
of a Poiseuille flow for a stationary ground plane or a Couette flow, for a moving ground plane.

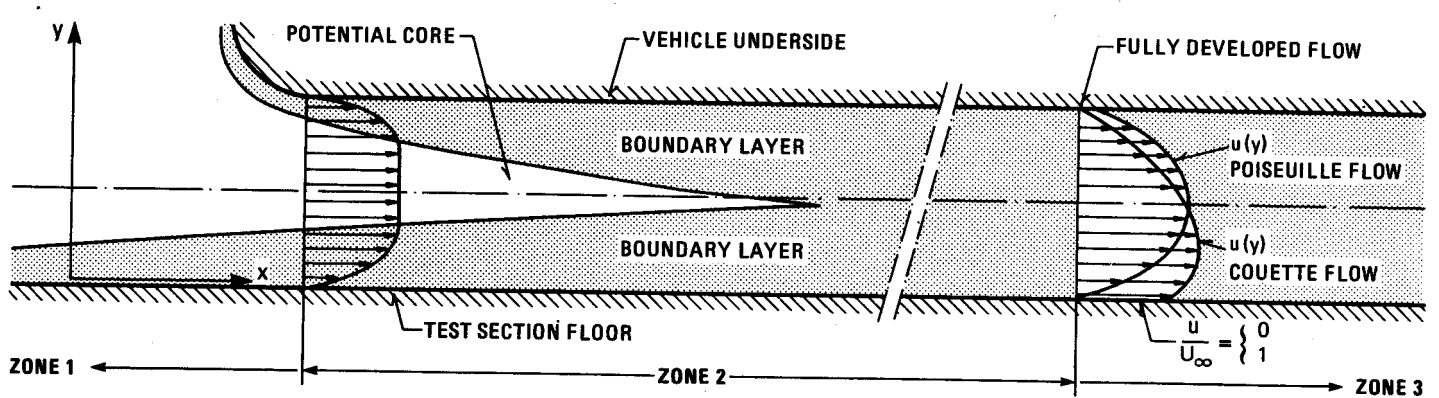

Figure 2.2: Three Zones of Automotive Underbody Airflow.

Mercker and Wiedemann state that the steady turbulent boundary layer in the wind tunnel is considerably thicker than the unsteady boundary layer generated at the road under the moving car. The ground-floor boundary layer restricts and alters the under-body flow of the ground vehicle, which produces flow different than that experienced in the real world. If parts of the vehicle are in the ground plane boundary layer region their aerodynamic coefficients are likely to be altered.

Systems that are currently used for ground simulation include, but are not limited to, basic fixed-floor wind tunnel, symmetric models, elevated ground plane, raised floor with leading edge suction, suction through a perforated floor, tangential blowing, a moving ground plane, rotation wheels with a fixed ground plane, rotating wheels with a moving ground plane.

In the 1930's a moving belt was introduced as a technique to simulate the ground moving under a vehicle. To adequately simulate the effects of the ground it is important to match the speed of the belt to the speed of the free stream air. The moving ground plane continuously feeds momentum into the flow, which compensates for the viscous 
losses of the boundary layer. However, this also increases the volume flux of air flowing in the channel created by the ground and the underbody of the vehicle. This increase in volume flow rate affects the external pressure distribution and the base pressure on the surface of the automobile. According to Hucho (1998) the proper measure is to use a belt that spans the entire width of the test section, with the belt causing tire rotation. To get a more realistic simulation the approaching boundary layer should be removed to avoid boundary layer relaxation effects on the belt (Mercker and Wiedemann, 1990).

However there are also methods in which the linear and rotational motions have been mechanically decoupled. In this technique, a belt approximately the width of the model can be used to simulate the linear motion, while the tires can be either rotated separately or neglected. This method is not as precise of a simulation as using the belt to drive the wheels; it is still a reproduction of the flow. It is important to note that the flow conditions near the wheels have limitations. Rotating the tire has the inherent difficulty of matching the speed of the tire to that of the belt, which is also matched to the air speed in the wind tunnel. Alternatives to using a belt exist due to the large effort required in the installation and maintenance of the moving belt. In addition, the moving belt is a simulation and therefore has some discrepancies with reality. Another reason for finding alternatives to the moving belt is to allow for instrumentation, i.e. an external under floor balance.

Mercker and Wiedemann (1990) suggest that the empty-test-section boundary layer be kept as small as possible to minimize its interference effects. This can be done with the use of a boundary layer scoop at the front of the vehicle, or a basic suction system that applies a slotted upstream suction to the ground plane. By removing part or 
all of the boundary layer, the thickness of the boundary layer is reduced. It re-grows from a smaller thickness, therefore is reduced when compared to the boundary layer thickness if no changes were present. Hucho (1998) provides another option, which is to place the model on a second floor, which is above the tunnel floor boundary layer. The boundary layer of this second floor would be smaller than that of the tunnel floor because the upstream floor length with respect to the model is much smaller.

Mercker and Wiedemann (1990) introduce a suction parameter, $C_{Q}$, as defined in Equation 2.1, where $s$ is the slot width, $v_{s}$ is the suction velocity, $U_{\infty}$ is the free stream velocity and $\delta_{1 x=0}$ is the boundary layer thickness removed by suction.

$$
C_{\dot{Q}}=\frac{\dot{Q}}{\dot{V}}=\frac{v_{s} s}{U_{\infty} \delta_{1 x=0}}
$$

Ground simulation using suction only at the leading edge of the model allows for boundary layer re-growth downstream of the suction slot. To prevent this growth, distributed suction through a perforated ground plane can be used. However, this method evokes some technical difficulties, the first of which is sealing the suction chamber and the force/moment balance to avoid erroneous readings. Another difficulty of distributed suction is whether to use constant suction or an asymptotic suction. Since the viscous layers merge to a fully developed flow, the displacement effects are asymptotically reduced and then the required suction reduces to zero as the distance, $x$, along the test section increases. In the case where a constant suction is applied there is some undesired three-dimensional effects created by continuity as $x$ is increased. In addition to the potential for three-dimensional effects, other drawbacks to boundary layer suction exist. These include a noticeable negative angle of attack (sink angle) induced by the vertical velocity created by the suction, and the volume flow sucked off of the boundary layer is 
not constant due to the transverse pressure gradient from the front of the model, which results in an additional three-dimensional effect along the ground plane.

Hucho (1998) considers the distributed suction controversial because the correct suction rate is difficult to determine. Also, the suction rate is determined to produce a specific displacement thickness in an empty wind tunnel, thus when the model is placed in the wind tunnel the suction is too large which results in changes in the velocity profile of the boundary layer. Another discrepancy in using the distributed suction is that the presence of the model changes the pressure distribution above the ground plane. This in turn causes an unknown change in the suction rate, and creates possible regions of blowing, negative suction. Typically the forward part of the model is where this negative suction will occur. Carr (1994) compared the results from stationary ground plane tests with distributed suction to moving ground plane tests. An increase in down-force was noticed in the suction case. When compared to the real-world data the distributed suction case had smaller differences than the moving ground plane tests. Carr also noticed that the influence of ground plane suction on drag was less than that of the moving ground plane, from which small drag increases were induced.

Another way to simulate the movement of the ground is to add mass and momentum to the gap flow, which can be done with tangential blowing. Air can be injected at the leading edge of the test section through either a series of nozzles or a slot. For every wind speed in the wind tunnel test section there is a specific jet speed required to achieve zero displacement thickness at any given location. To produce a real-world representation of the flow field at the rear of the vehicle it is necessary to "over-blow" at the leading edge, creating unrealistically high drag forces on the front wheels. Another 
drawback to the use of tangential blowing is that the wall jet does not continuously add momentum to the flow, so the proper momentum flux is only met at a single stream-wise position. Hucho (1998), recommends using tangential blowing for vehicles that have either extremely low ground clearance, very low drag coefficent $(<0.25)$ and a lift coefficient approximately equal to zero, or extremely long vehicles. Mercker and Wiedemann (1990) considered tangential blowing to meet the requirements of the different zones of the flow. But the authors mention that there is a costly technical effort required to use tangential blowing in full-scale facilities.

The mirror image technique creates a centered streamline, which simulates the existence of the ground plane. There are two main disadvantages to this technique, the first of which is that vortices created by the model (behind the wheels) induce a random oscillation into this imaginary ground surface. The other important drawback of using the mirror image technique is that two models need to be generated and placed in the wind tunnel, which requires either smaller models or larger test sections. Another method used to simulate the ground would be to raise the model above the floor. Raising the model above the boundary layer displacement thickness would eliminate the viscous effects of the boundary layer. However, this method introduces systematic errors in the incorrect ground clearance, which may affect the airflow, particularly around the wheels. Therefore, the drag and lift measurements may not be accurate.

The easiest way to simulate the ground effects is simply use the floor of the wind tunnel test section as a stationary ground plane. However, the boundary layer does deform the flow around the model, and separates in front of the wheels as well as in any adverse pressure gradient under the vehicle. In some instances, components near the 
ground will appear less effective than in real-world conditions. In testing conducted by Beauvais, Tignor and Turner (1978), comparisons were made between fixed ground plane, moving belt, and an elevated model, and Table 2.1 shows the results of this testing. From these results for automobile testing, it can be argued that acceptable errors can be achieved with the fixed ground plane, especially if the testing is not concerned with the lift force(s).

Even though the majority of the discussion on ground simulation was for automobile testing, similar ideas can also be used when discussing motorcycle testing. But, it is important to note the differences between automobiles and motorcycles. The major difference between the two vehicles is their shape and size. The motorcycle does not have near the concern of airflow under it, due to the smaller underbody surface. However, the tires of the motorcycle represent a greater percentage of the total drag of the motorcycle, and therefore more attention needs to be placed on the wheels, much like an open-wheeled racing automobile.

Table 2.1: Summary of Test Results from Beauvais, et al (1978).

\begin{tabular}{|c|c|c|c|c|}
\hline & $\mathrm{C}_{\mathrm{L}}$ & \% Variation & $\mathrm{C}_{\mathrm{D}}$ & \% Variation \\
\hline Full Scale Vehicle & 0.552 & -- & 0.540 & -- \\
\hline Fixed Ground Plane & 0.596 & 8.0 & 0.548 & 1.5 \\
\hline Moving Belt Ground Plane & 0.417 & -24.5 & 0.510 & -5.6 \\
\hline $\begin{array}{c}\text { Fixed Ground Plane } \\
\text { (Model at }+0.125 \text { in.) }\end{array}$ & 0.570 & 3.3 & 0.553 & 2.4 \\
\hline
\end{tabular}




\subsection{Wind Tunnel Blockage}

In wind tunnel testing, it is important to consider the size of the model to be tested with respect to the size of the test section size. There is a trade off that needs to be made when performing wind tunnel tests; one side of the argument is to reduce operation costs. The other is to increase model size for scale issues, more accessibility for instrumentation, etc. Not only is the physical size of the model important, but so is the size of the wake created by the model. According to Barlow, Rae and Pope (1999) it is also important to consider the momentum effects outside the wake, when separated flow is present. These effects are produced by a lateral-wall constraint on the wake and results in a lower wake pressure, which in turn produces a lower base pressure on the model than would occur in free air. The standard parameter in discussing the size of the model is the blockage ratio, which is defined as the ratio of the model frontal area over the test section cross sectional area. Typical blockage ratios used are $0.05,0.10$, and 0.20 in some cases, however Katz and Walters (1995) suggest avoiding a blockage ratio that is higher than 0.07 .

Some corrections for wind tunnel blockage can be formulated, however they can merely provide some estimation of the wall effects. Additional effects, such as an altered boundary layer transition, turbulence levels, and deforming streamlines may also exist.

One effect of test section blockage is an increase in air speed through the narrowed passage between the model and the walls. The increased speed artificially raises the values of the aerodynamic coefficients. This discrepancy is difficult to measure because of the desire to avoid disturbances, such as pitot-static tubes, in the airflow close to the model. In high blockage ratio testing the interaction between the walls of the test 
section and the model surface result in effects similar to ground effects experienced in aircraft testing. Several mathematical formulas have been developed to account for test section blockage in the measurement of drag.

The following corrections, Equations 2.2 and 2.3, for drag coefficient were formulated by Barlow, Rae and Pope (1999) where $C_{D}$ is the corrected drag coefficient, $C_{\text {Dmeas }}$ is the measured drag coefficient, $A$ is the model frontal area, $S$ is the cross sectional area of the test section, and $C_{P \min }$ is the minimum pressure on the model.

$$
\begin{gathered}
C_{D}=\frac{C_{D_{\text {meas }}}}{\left(1+\frac{A}{4 S}\right)^{2}} \\
C_{D}=\frac{C_{D_{\text {meas }}}}{\left(1-C_{P_{\text {min }}}\right)}
\end{gathered}
$$

Barlow, Rae and Pope (1999) define an approximate blockage correction factor, $\varepsilon_{t}$, where $A$ is the model frontal area and $S$ is the tunnel cross sectional area:

$$
\varepsilon_{t}=\frac{A}{4 S}
$$

\subsection{Turbulence Control and Velocity Profile}

When testing in wind tunnels, it is desirable to have uniform velocity profiles in the test section. Errors are introduced into measurements when the velocity profiles are non-uniform, though small fluctuations are tolerable. A major influence of the uniform nature of the air is the level of turbulence present in the wind tunnel. Objects in the flow, such as the propeller and its mountings, the spinner, turning vanes, etc, are the primary cause of turbulence in the wind tunnel. Minimizing the air speed in areas of the wind 
tunnel other than the test section, and/or the installation of damping screens or honeycomb guide vanes can reduce this turbulence. According to Dryden and Abbott (1949), despite the ability to reduce the turbulence, the noise generated by the motor and other acoustic sources place a lower limit on the turbulence level.

When using damping screens, screens with a high-pressure drop coefficient, $K$ defined in Equation 2.5, generally are not as effective at damping the turbulence and spatial variations than low $K$ screens. In the following relationship $\Delta P$ is the pressure drop over the screen, $\rho$ is the air density and $U$ is the velocity of the air.

$$
\Delta P=\frac{1}{2} \rho U^{2} K
$$

Therefore it is more beneficial to use a series of low-pressure drop screens instead of one high-pressure drop screen. The effectiveness of the damping screens has been described in several different ways, by the formulas shown below. The Prandtl Damping Formula is shown in Equation 2.6 and Equation 2.7 is the Collar Damping Formula (Schubauer, Spangenberg and Klebanoff, 1950). These two damping formulae are approximations of experimental data. In both of these equations $f$ is the damping factor .

$$
\begin{aligned}
& f=\frac{1}{1+K} \\
& f=\frac{2-K}{2+K}
\end{aligned}
$$

The Dryden and Schubauer Damping Formula, Equation 2.8, fits experimental results better than the previous damping formulae, thus it is a more useful relationship (Schubauer, Spangenberg and Klebanoff, 1950).

$$
f=\frac{1}{\sqrt{1+K}}
$$


The damping screens also provide assistance in improving the flow quality across the width and height of the wind tunnel, allowing for a more uniform velocity profile. Figures 2.3 and 2.4 show the comparisons made by Smith, et. al. (1997) of the velocity profiles of the WVU Closed Loop Wind Tunnel. Notice the reduction in maximum air speed as well as the reduction in the velocity fluctuations for the case with the damping screen (Figure 2.4). Another important note about the use of both damping screens and flow straightening honeycomb mesh is that the effectiveness of these devices are greatly altered by minor damage. Therefore great care must be taken in the installation and maintenance of these devices.

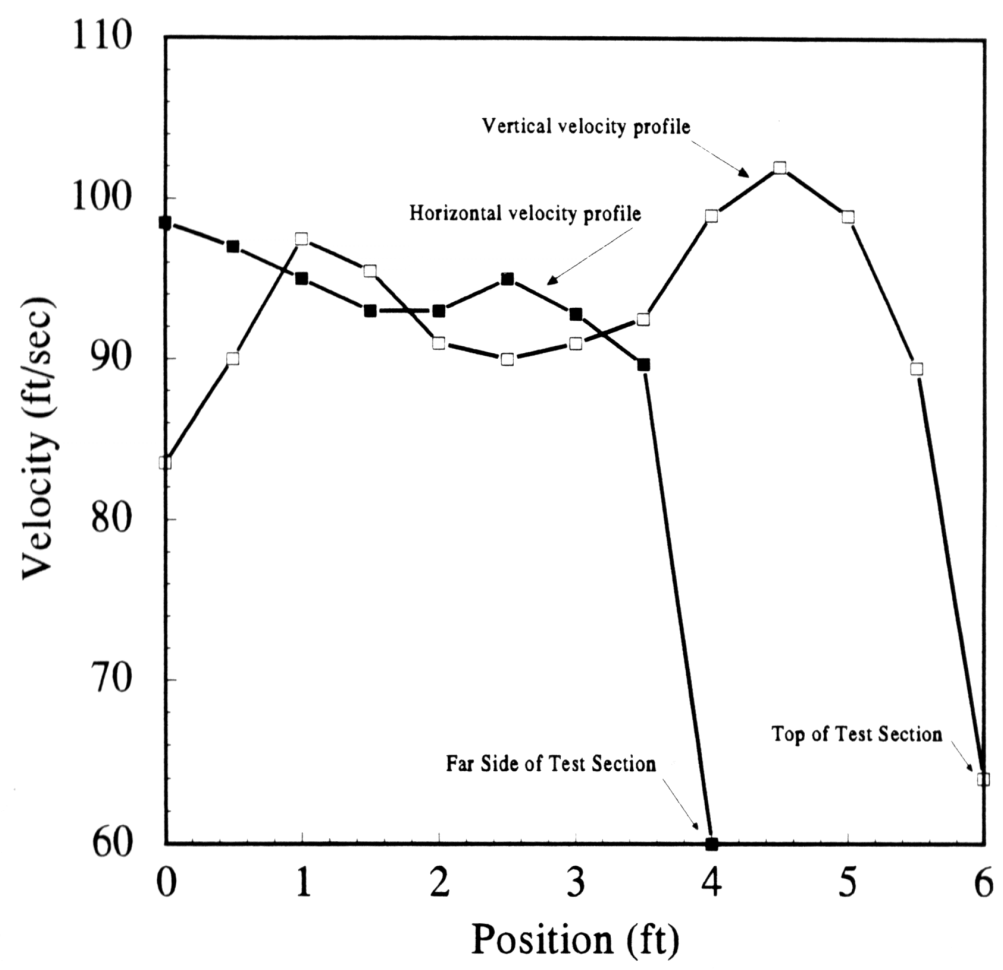

Figure 2.3: Horizontal and Vertical Velocity Profiles Without Damping Screen 


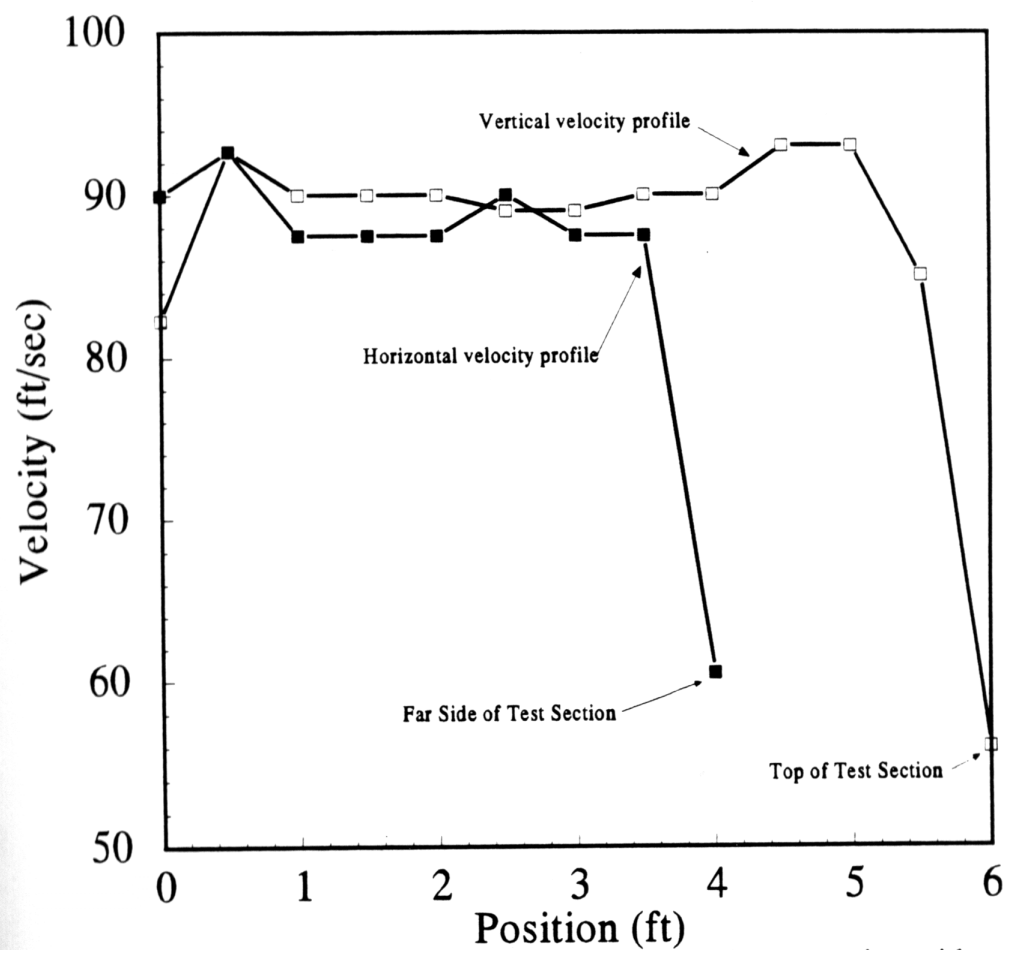

Figure 2.4: Horizontal and Vertical Velocity Profiles With Damping Screen

\subsection{Vortex Generators}

There are two types of aerodynamic drag encountered by road vehicles: friction drag and pressure drag. In many automobiles, the air separates near the rear of the vehicle, increasing the drag due to the separated wake of the fluid and the pressure drag. Several different methods of automobile wake reduction have been suggested, including the use of more aerodynamic shapes, powered suction, and boundary layer reenergization. The power required for suction to generate a noticeable change in drag is far greater than the capacity of the engine of the automobile. Thus, the powered suction technique is impractical.

To avoid long streamlined rear sections of vehicles it is necessary to re-energize the boundary layer. The boundary layer can be re-energized by mixing some of the free- 
stream air with the boundary layer air. This mixing increases the energy of the air in the boundary layer delaying boundary layer separation and the size of the wake produced by the vehicle. Some vehicles employ wing-like airfoils as turning vanes to assist in directing the flow, thus reducing the wake region of the automobile. Similarly, a vortex generator is a device that can be used to re-energize the boundary layer, delaying flow separation, as seen in Figure 2.5. These vortex generators (VGs) create vortices with a diameter of up to five device heights above the installed surface (Wheeler, 1991). These vortices mix the high energy free stream air with the lower momentum boundary layer air. The increase in boundary layer momentum effectively delays the onset of separation and allows for higher angles of attack than the unmixed flow.

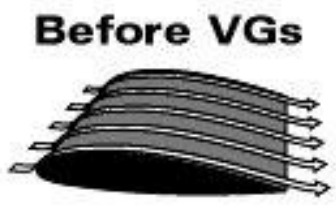

Smooth airflow

After VGs

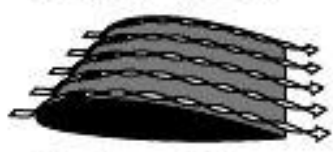

Vortex airflow

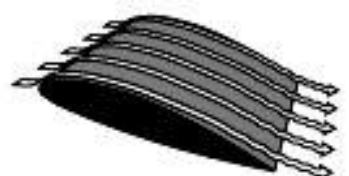

Boundary layer begins to separate

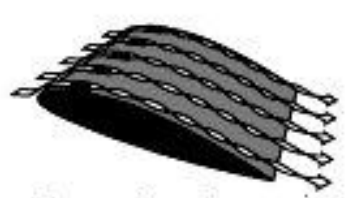

Boundary layer energized by vortices

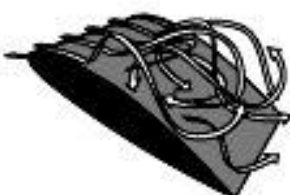

Wing stalls

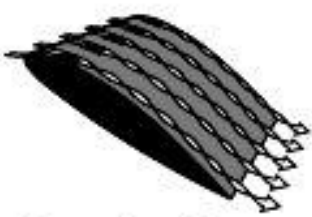

Boundary layer remains attached

Figure 2.5: Vortex Generator Effects of Airflow over 2-D Airfoil.

Vortex generators are essentially a protrusion into the free stream air that sheds a trailing vortex, or vortices, into the boundary layer downstream. There are several different types of vortex generators 1) forwards wedge, 2) backwards wedge, 3) counterrotating vanes, and 4) single rotation vanes, shown in Figure 2.6. The four types of vortex generators all add energy to the flow by creating a vortex, or a pair of counter- 
rotating vortices, which mixes the low energy boundary layer air with the high-energy flow of the free stream, which delays flow separation. However, these vortex generators do have a drag penalty but this penalty is often less than the drag reduction potential they offer. Therefore a net drag reduction can be experienced. Due to this drag penalty, vortex generators are typically not used in applications where the benefits are only realized for a small portion of the operating time, minimizing the total drag.

The vortex generators can be applied in a series arrangement to cover the surface of large shapes. The goal of designing components using vortex generators is to maximize component performance, and minimize the number of devices. This optimization process presents difficulties due to the large number of parameters involved in a general configuration of vortex generators. These include chord length, c, span, h, angle of attack with respect to the free-stream direction, $\alpha$, and other parameters dealing with the vane cross-section and axial profile, as well as the geometry of the array formation and flow conditions (Wendt, 1994).

The height of the vortex generator is generally above the boundary layer thickness. However, some vortex generators that have a height considerably less than the boundary layer thickness, these are Sub-Boundary Layer Vortex Generators (SBVGs), or Micro-Vortex Generators (MVGs). These SBVGs were developed as a way to minimize device drag while maintaining flow control, optimizing device effectiveness. The vortices generated by the SBVGs are weaker than those generated by boundary layer sized VGs, but are typically strong enough to maintain adequate flow control. Ashill, et al. ${ }^{1}$, in a study of SBVGs experimented with forward wedges, joined counter-rotating vanes, and counter-rotating vanes spaced apart by one device height. Ashill noticed that 


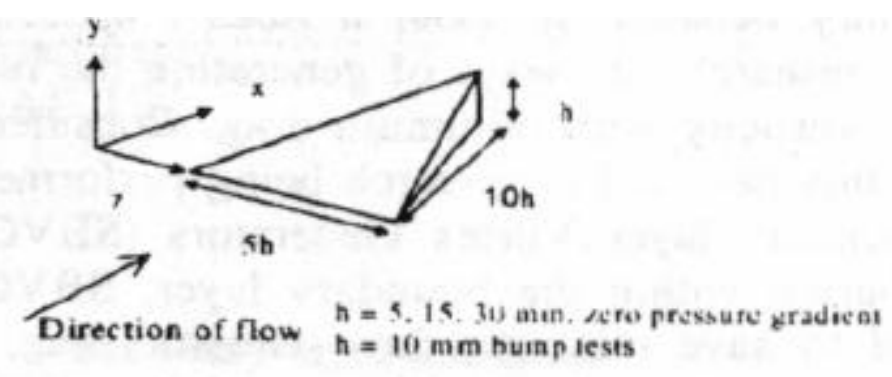

'Forwards wedge'

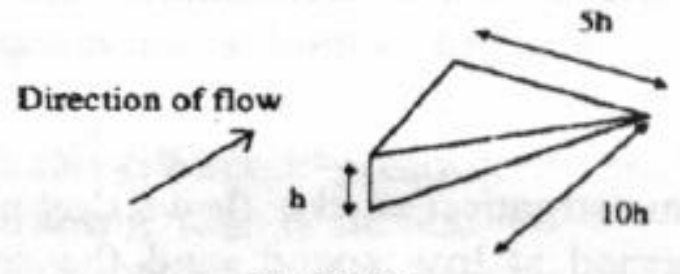

$\mathrm{h}=30 \mathrm{~mm}$

\section{'Backwards wedge'}

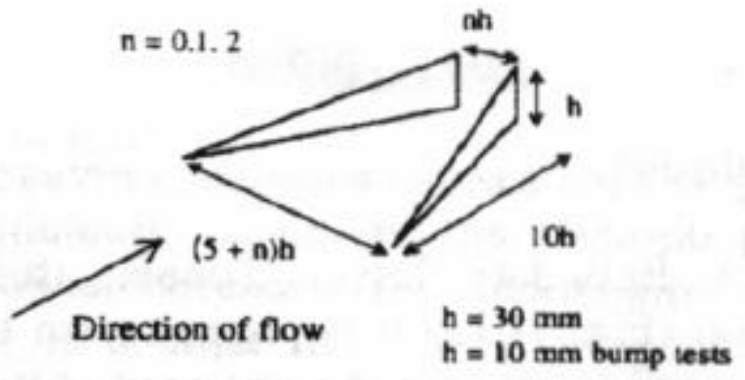

Counter-rotating vanes $(n=0$ joined vanes $)$

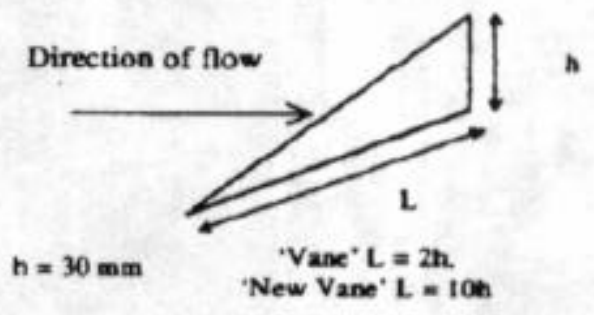

Single rotation vanes

Figure 2.6: Types of Vortex Generators 
the counter-rotating vanes spaced by one device height were the most effective in the reduction of flow separation, which corresponds to the devices that had the lowest vortex decay rate of the three types studied.

Two important mechanisms of the convectiveness of the vortices are the mixing of free-stream air with boundary layer air and the secondary flow control. The vortex mixes high-energy fluid of the free-stream with the slower moving fluid of the boundary layer, thinning the downstream region of the boundary layer. The overall effect of vortex mixing is to promote re-energization of the boundary layer fluid and extend the layer attachment. The rotational orientation of the vortex may also be used to counter boundary layer thickening due to the cumulative convective effects of secondary flows (Wendt, 1994). All types of vortex generators continuously add momentum to the boundary layer, re-energizing the fluid and countering the natural boundary layer growth (Tai, 2002). The optimum placement of an array of VG's is mainly determined through trial and error experiments, but CFD techniques can be used as an alternative to the experimentation.

The counter-rotating vortex generators designed by Mr. Gary Wheeler produce vortices that have stronger flow control than typical VGs. The arched shape of the Wheeler VG generates a larger vortex with minimal increase in the parasitic device drag experienced by other vortex generators. The counter-rotating jets typically produce higher circulation than co-rotating jets (Wheeler, 1984). Equation 2.9 mathematically models the vortex generated, where $\Gamma$ is the measured circulation, $\omega$ is the maximum stream wise vorticity, $R$ is the cross plane distance from the center of the vortex.

$$
\Gamma *\left[1-\exp \left(\frac{-\pi * \omega}{\Gamma}\right) * R^{2}\right]
$$


Results from Ashill's testing show that the joined counter-rotating vanes create the strongest vortices and the forward wedge generates the weakest vortex, in both experimental and CFD code, as listed in Table 2.2.

Table 2.2: Experimental and CFD Strengths of Vortices as Determined by Ashill, et al.

\begin{tabular}{|c|c|c|}
\hline \multicolumn{3}{|c|}{ Non-Dimensional Circulation, $\Gamma / \mathrm{u}_{\tau} * \mathrm{~h}$ (Measured 5 device heights downstream) } \\
\hline Device & Experimental & CFD \\
\hline Forward Wedge & 15.04 & 13.64 \\
\hline Joined Counter-rotating vanes & 35.65 & 27.75 \\
\hline Counter-rotating vanes, 1h spacing & 27.57 & 24.64 \\
\hline Counter-rotating vanes, 2h spacing & 25.34 & 18.86 \\
\hline
\end{tabular}




\subsection{Experimental Apparatus}

This chapter describes the equipment and facilities used for this research at West Virginia University and in Old Dominion University's Langley Full Scale Wind Tunnel. This covers discussion of the model, vortex generators and test instrumentation, including the calibration of instruments.

\subsection{Tul-Aris Model and Vortex Generators}

Dr. Robin Tuluie, designer of the Tul-Aris, provided a full-scale model of the motorcycle for this project. The model consists of a hand built metal frame, which holds the actual racing fairings of the 2001-Version Tul-Aris in their proper place. The fairings are composite structures with a smooth surface; there was a small portion of racing damage on the left side of the lower fairing. This damage was repaired using body putty. It was determined that this minor damage would have little effect on the results since a relative drag difference was the primary concern. Figures 3.1 and 3.2 show the side and top views of the Tul-Aris model used for testing in this research. Pressure taps were applied to the left-hand side of the model: 8 taps on the upper fairing and 30 taps on the lower fairing. Figure 3.3 illustrates the locations of the pressure taps.

It was noticed during Phase I of testing that the lower fairing of the model had a considerable amount of movement while air was flowing over it. After completion of Phase I a second support was added to the lower fairing. This support alleviated most of the vibrations during testing. The stiffened model was then used for Phases II and III.

When deciding what type of vortex generator to use, several types were considered including: sail type, forward and backward wedges and counter-rotating vanes 
(please refer to Figure 2.5 for pictures of the different types of VG's). From testing conducted by Ashill (2001) it was determined that the use of the counter-rotating pair of vanes created the highest circulation and therefore were more desirable to use in this application. The commercially available vortex generators that were used have a device height of 0.5 inches, as shown in Figure 3.4.

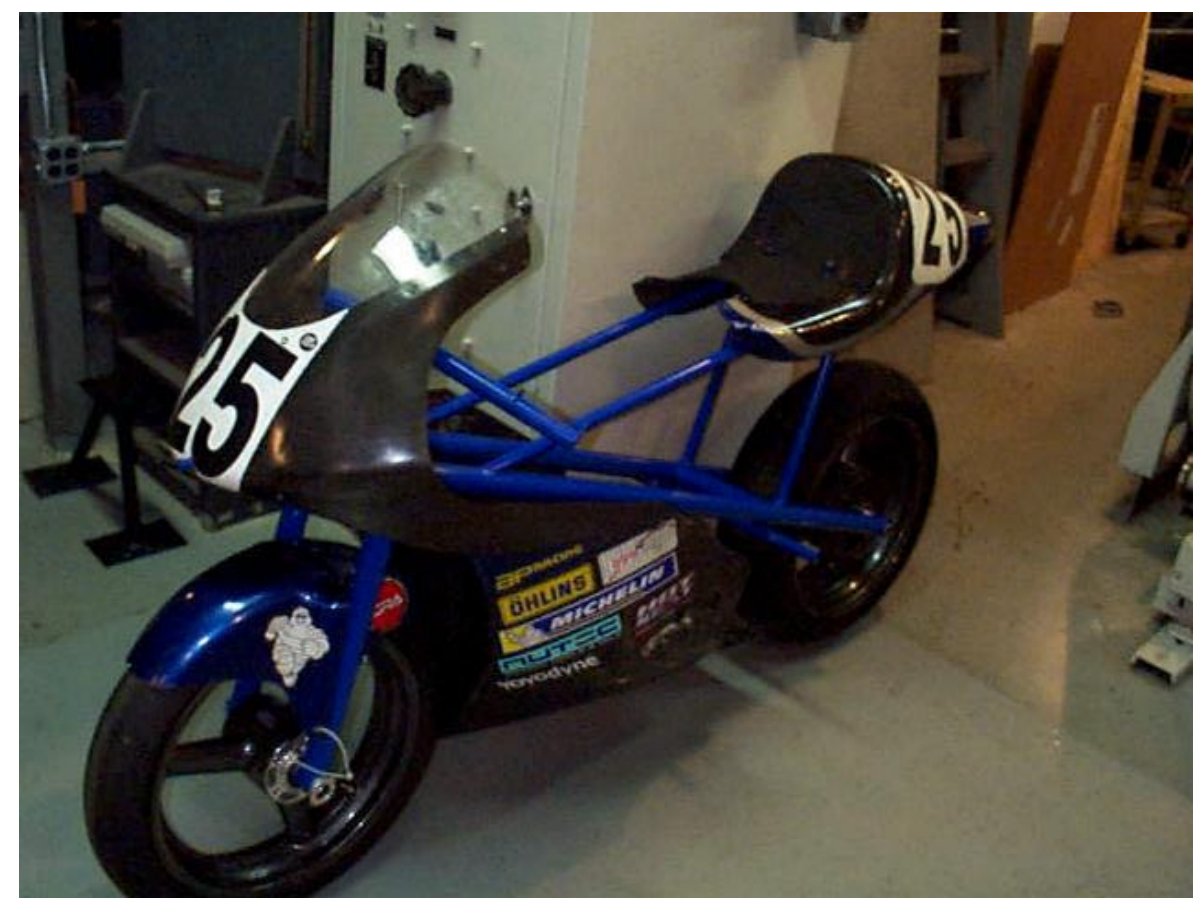

Figure 3.1: Side View of Tul-Aris Model, Without 'Dummy' Rider. 


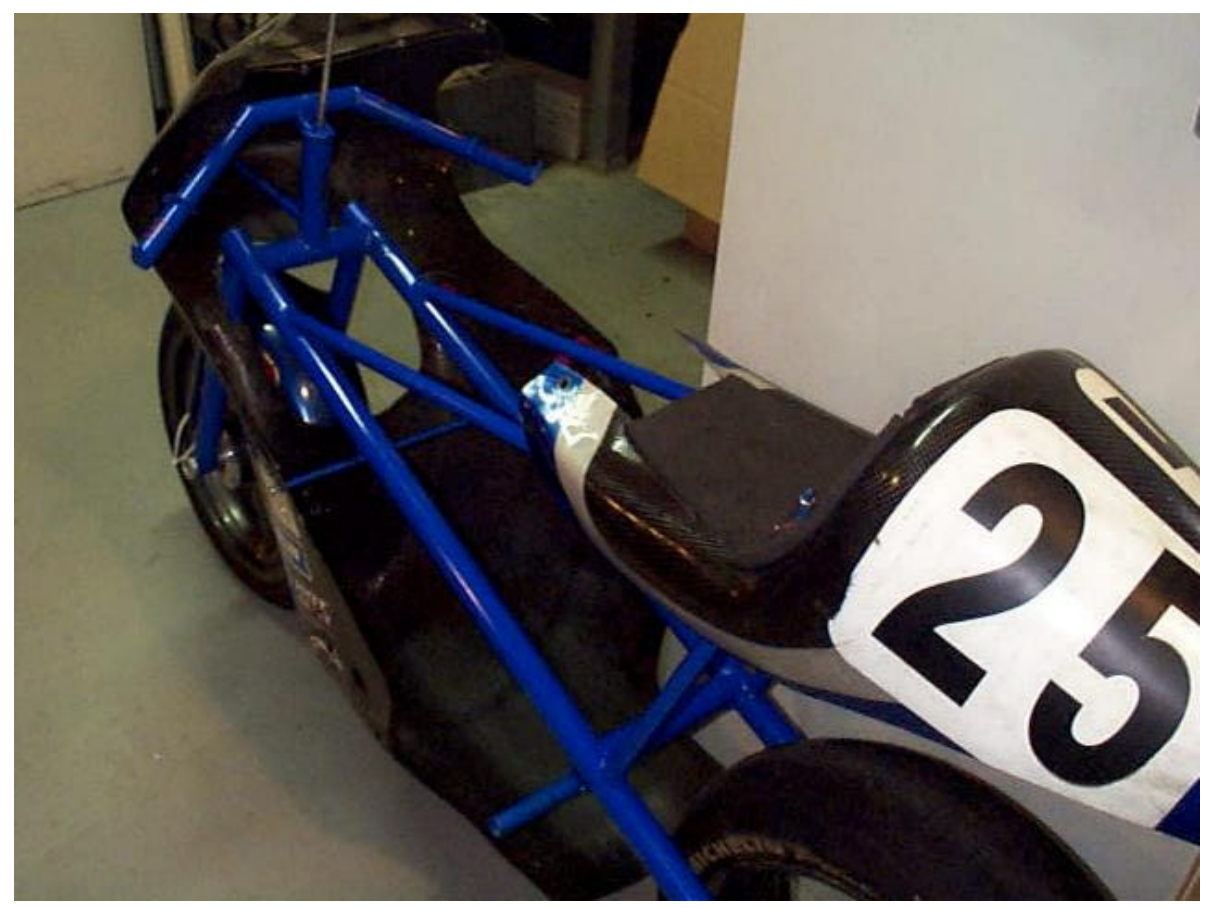

Figure 3.2: View inside Tul-Aris Model, Without Rider.

These vortex generators were also modified, through a simple machining process, to have device heights of 0.25 and 0.125 inches, shown in Figures 3.5 and 3.6, respectively. Discussion on placement of the vortex generators also occurred, which is covered in the Section 4.1: Experimental Procedure of this thesis. 


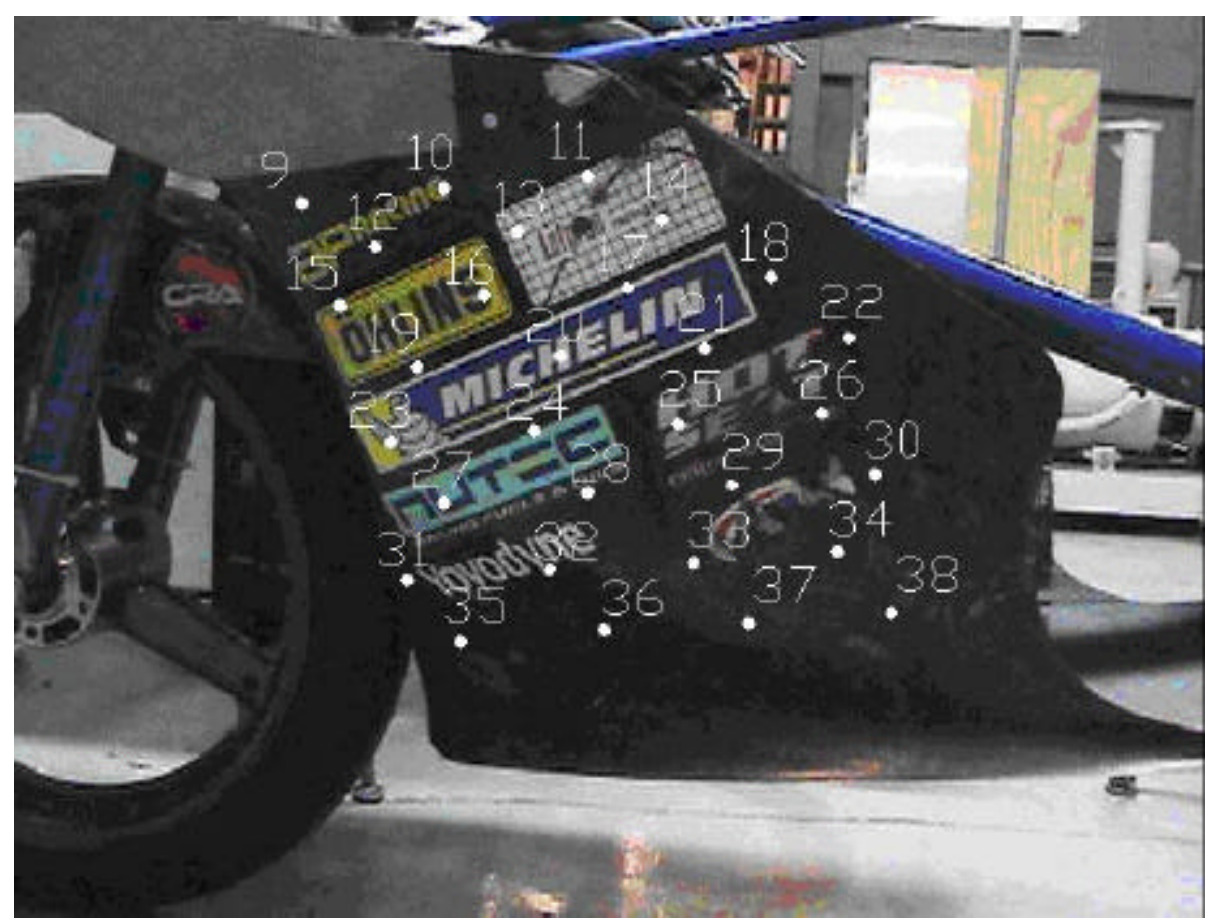

Figure 3.3: Picture of Pressure Tap Locations on the Lower Fairing of the Model.

A.

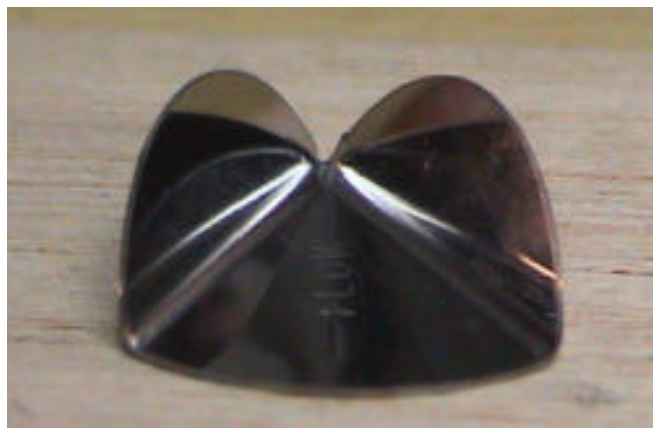

B.

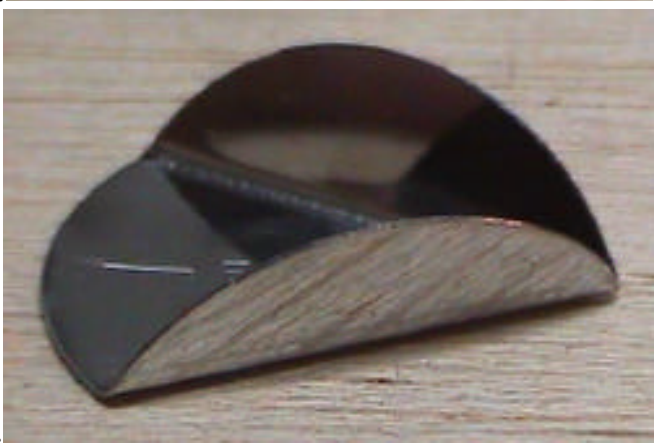

Figure 3.4: 1/2-inch Vortex Generator provided by Mr. Gary Wheeler.

A. Front View B. Side View 


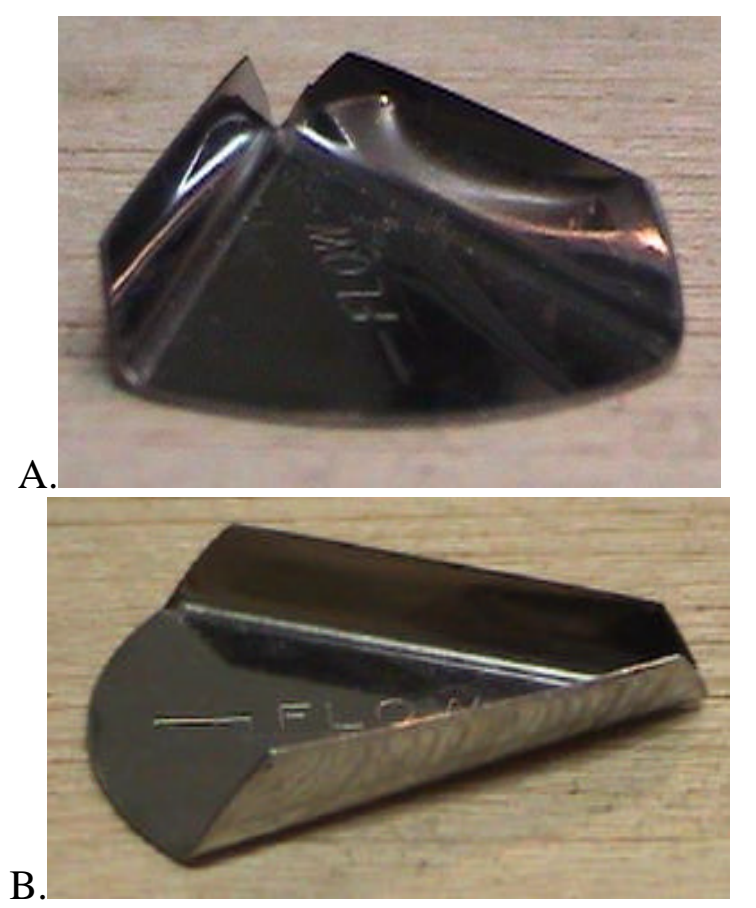

Figure 3.5: Modified 1/4-inch Vortex Generator.

A. Front View B. Side View

A.

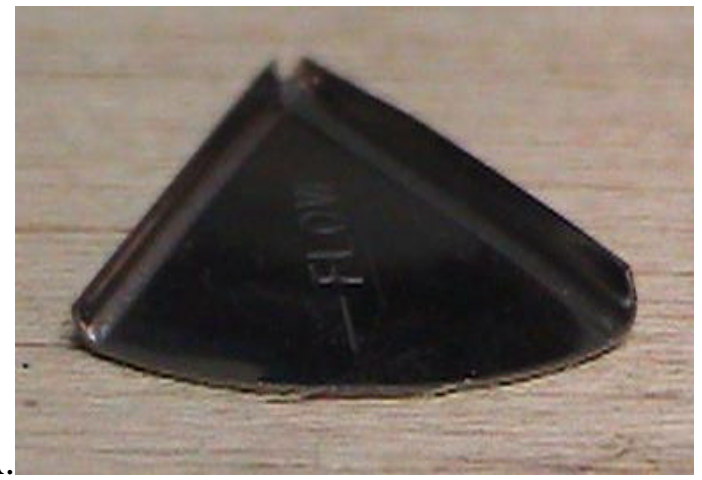

B.

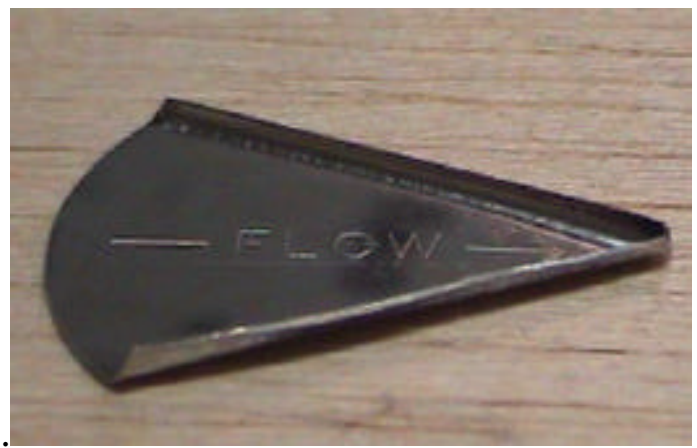

Figure 3.6: Modified 1/8-inch Vortex Generator.

A. Top View B. Side View 


\subsection{Testing Apparatus used at West Virginia University}

The 4' by 6' modified test section in the WVU Closed Loop Wind Tunnel was selected for use for the majority of testing conducted at WVU. To ensure the test section had a uniform horizontal velocity profile a diffuser was added to the wind tunnel downstream of the 4' by 6 ' test section, Figure 3.7. Figure 3.8 shows the comparison between the Smith, et. al. (1997) non-dimensional horizontal velocity profile with the velocity measured after the addition of the diffuser and without the flow straightening screen in place. See Figure 3.7 for placement of this screen. Figure 3.9 shows the same comparison as in Figure 3.8, but the comparison is with the screen in place as shown.

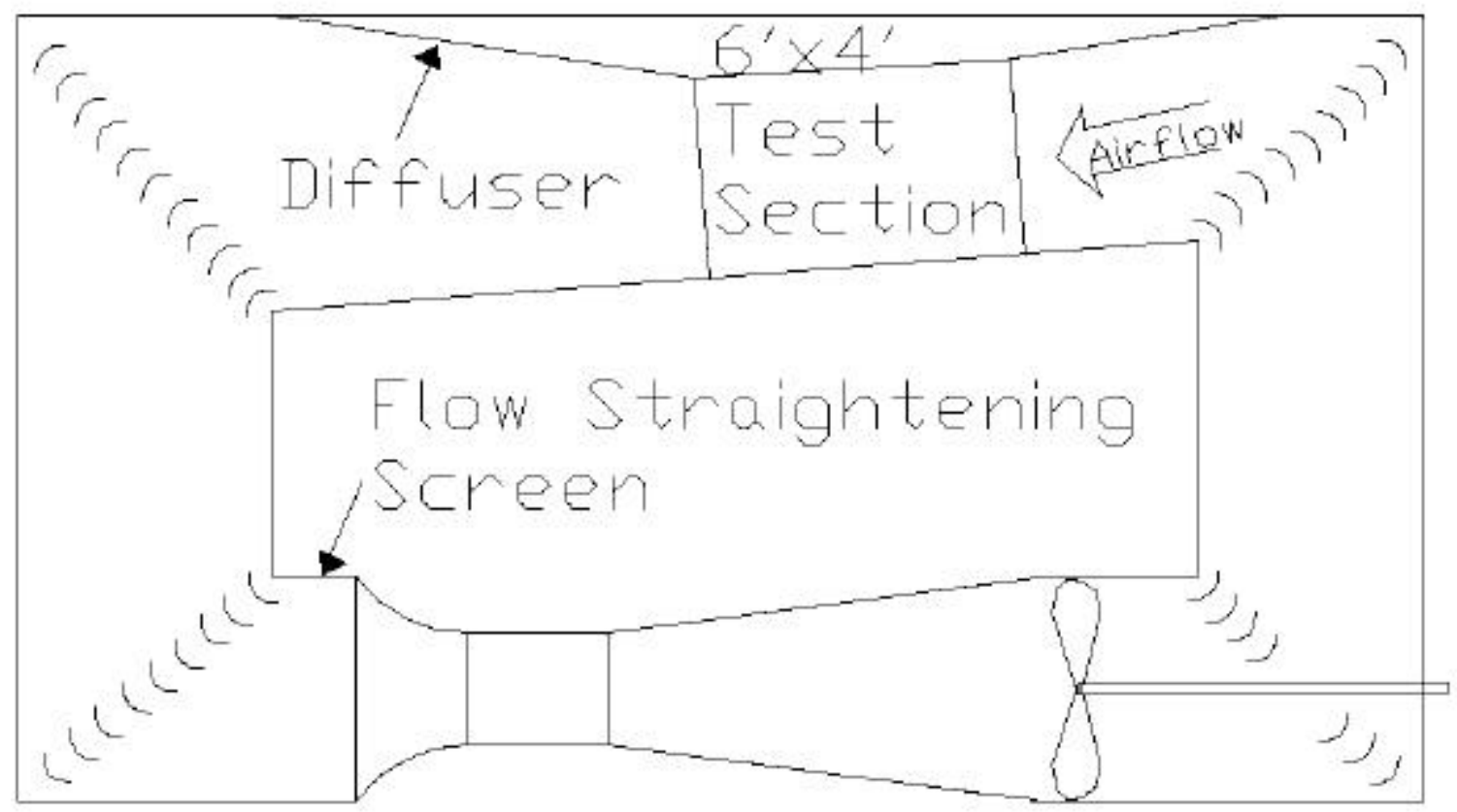

Figure 3.7: Schematic of WVU Closed Loop Wind Tunnel With Locations of Flow Straightening Screens and Diffuser. 
The data was collected for the "with diffuser" tests using a pitot-static tube. Measurements were taken near the leading edge of the large test section at a height of $3 \mathrm{ft}$ from the ground plane. Figure 3.8 shows that adding the diffuser to the wind tunnel allowed for a more uniform velocity profile on the outside of the test section. However, due to the upstream turn the addition of the diffuser to the system increased the momentum effects of the turning airflow. Installing the flow straightening screen to the system decreased the effects of inertia experienced in the test section, slightly improving the velocity profile of the test section.

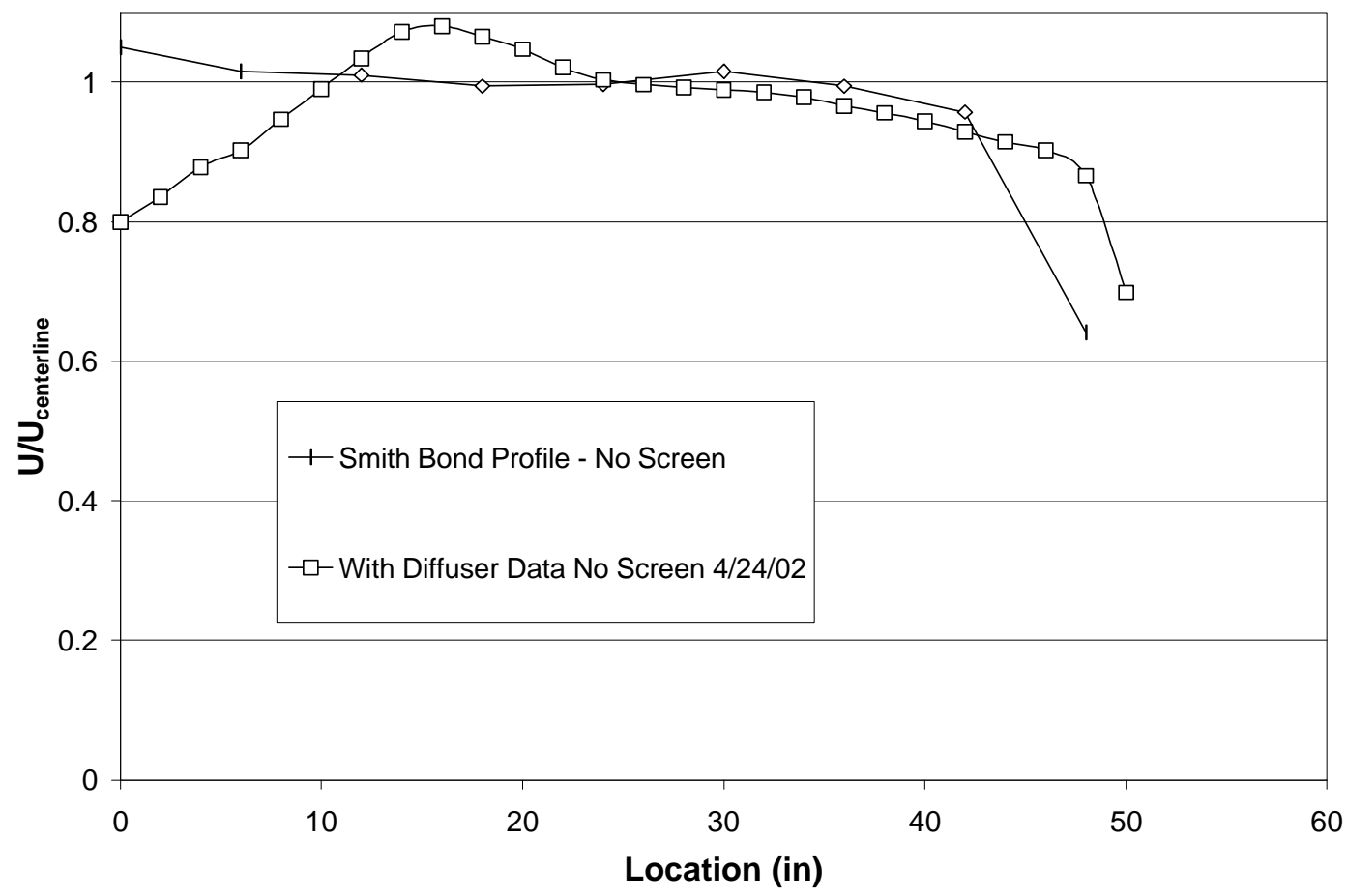

Figure 3.8: Comparison of Non-Dimensional Horizontal Velocity Profiles with No Flow Straightening Screens and Diffuser. 


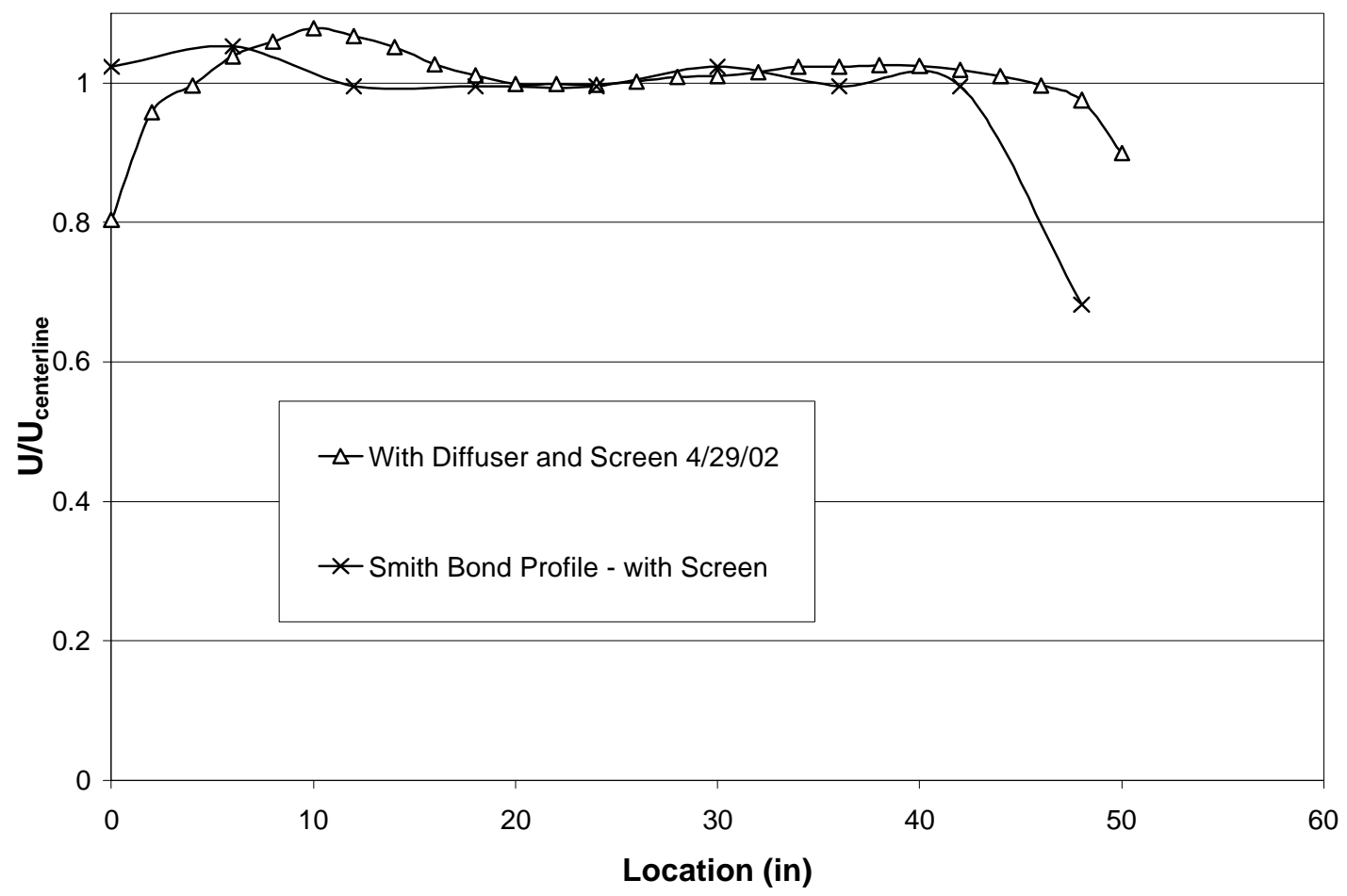

Figure 3.9: Comparison of Non-Dimensional Horizontal Velocity Profiles With Flow Straightening Screen.

A blockage ratio of 0.208 was present during testing in the test section; Figure 3.10 was used to determine the frontal area of the motorcycle. After adjusting the velocity for blockage the coefficient of drag does not adequately agree with the Langley Full Scale Tunnel testing. Therefore the Barlow, Rae and Pope (1999) method of accounting for blockage in wind tunnel testing, Equation 3.1, was used to account for this high blockage ratio. In this relationship $C_{D}$ is the adjusted drag coefficient, $C_{\text {Dmeas }}$ is the measured drag coefficient, $A$ is the model frontal area, and $S$ is the test section cross sectional area.

$$
C_{D}=\frac{C_{D_{\text {meas }}}}{\left(1+\frac{A}{4 S}\right)^{2}}
$$


Similarly, the type of ground simulation technique used in this testing was determined to be the use of the stationary tunnel floor with a slightly elevated model, approximately $1 / 4$ inch from the tunnel floor. This was found to be the best option due to the limited cost and simplicity in installation. According to Beauvais, et. al. (1978), Table 2.1, this would result in a variation of approximately $3 \%$ in the lift and drag components. Again, the ground simulation conditions are constant between tests so the measured change in drag is not a result of the ground simulation technique used during testing. However, effects of the non-rotating wheel on the vortex generators can not be quantified at this time. 


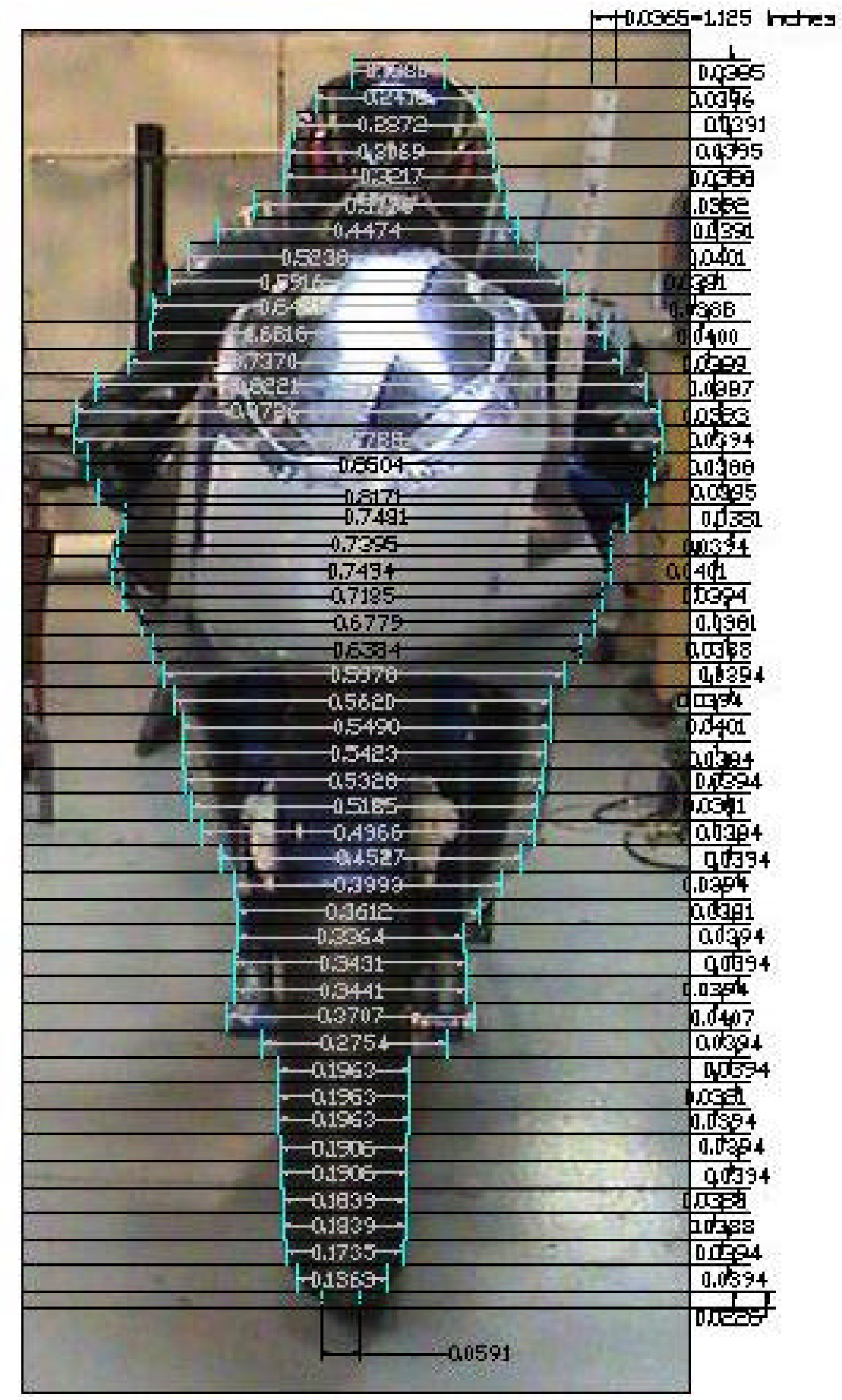

Figure 3.10: Frontal View of Tul-Aris Model for Frontal Area Determination. 
Three types of measurements were taken during the testing at West Virginia University; they are drag force, air temperature and surface pressure at various locations. The primary measurement, drag force, was recorded through a S-Type Load Cell acquired from Omegadyne. This load cell was given an excitation voltage of 12 Volts; the calibration of this load cell was determined from applied weights. Figure 3.11 shows the calibration curve for the Omegadyne load cell, correlating the force to voltage. Due to the tendency of the temperature of the air in the wind tunnel to increase, a Type $\mathrm{J}$ thermocouple, calibrated by the manufacturer, was used to account for this rise in temperature through the density of the air. The model surface pressure measurements were taken using a Scanivalve system that incorporated a single pressure transducer, with a range of 1 psid with an excitation voltage of $12 \mathrm{~V}$, to measure up to 48 pressure taps sequentially. Using a system built at WVU, shown in Figure 3.12, the pressure transducer was calibrated by applying a pressure to both the water manometer and the Scani-Valve using a vacuum pump then sealing the system with a valve. After incrementing the pressure back to atmospheric pressure the calibration was complete. The calibration curve is shown in Figure 3.13 along with the linear fit to the data. 


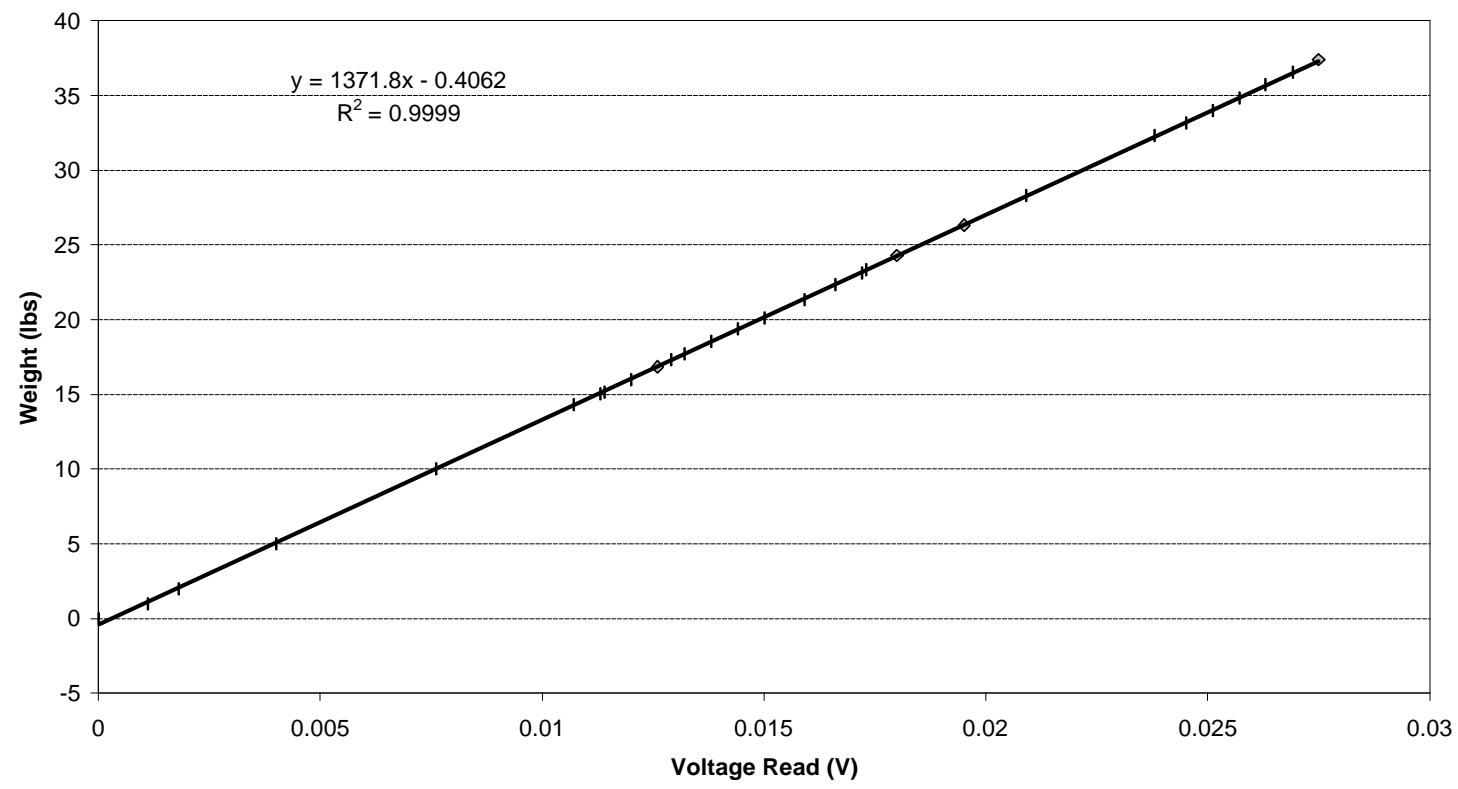

Figure 3.11: Calibration Curve for the Omegadyne Load Cell.

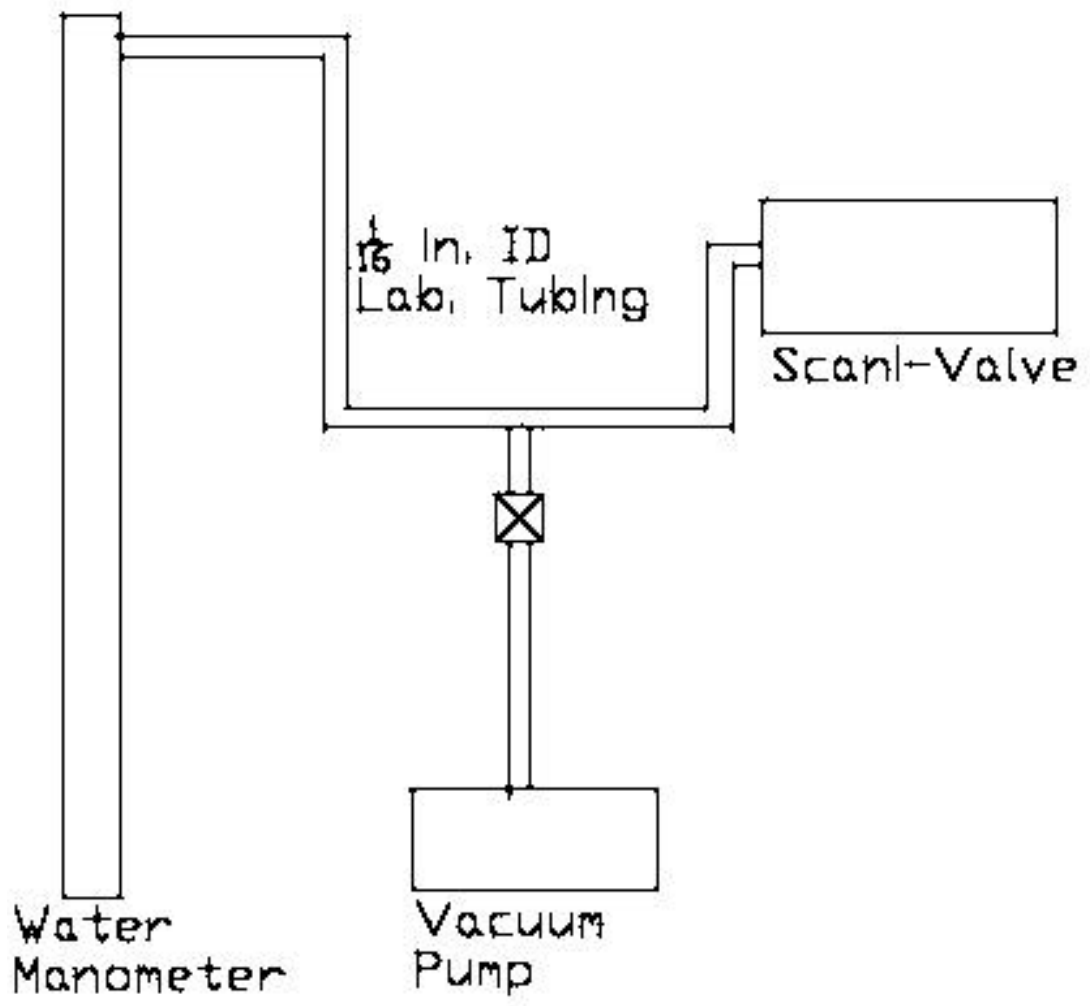

Figure 3.12: Sketch of Device used to Calibrate Scani-Valve. 
These three devices all produce a voltage output, which enabled the use of a computerbased data acquisition system with a 12-bit data acquisition card. The LabVIEW code that was used to control the data acquisition is shown in Appendix A.

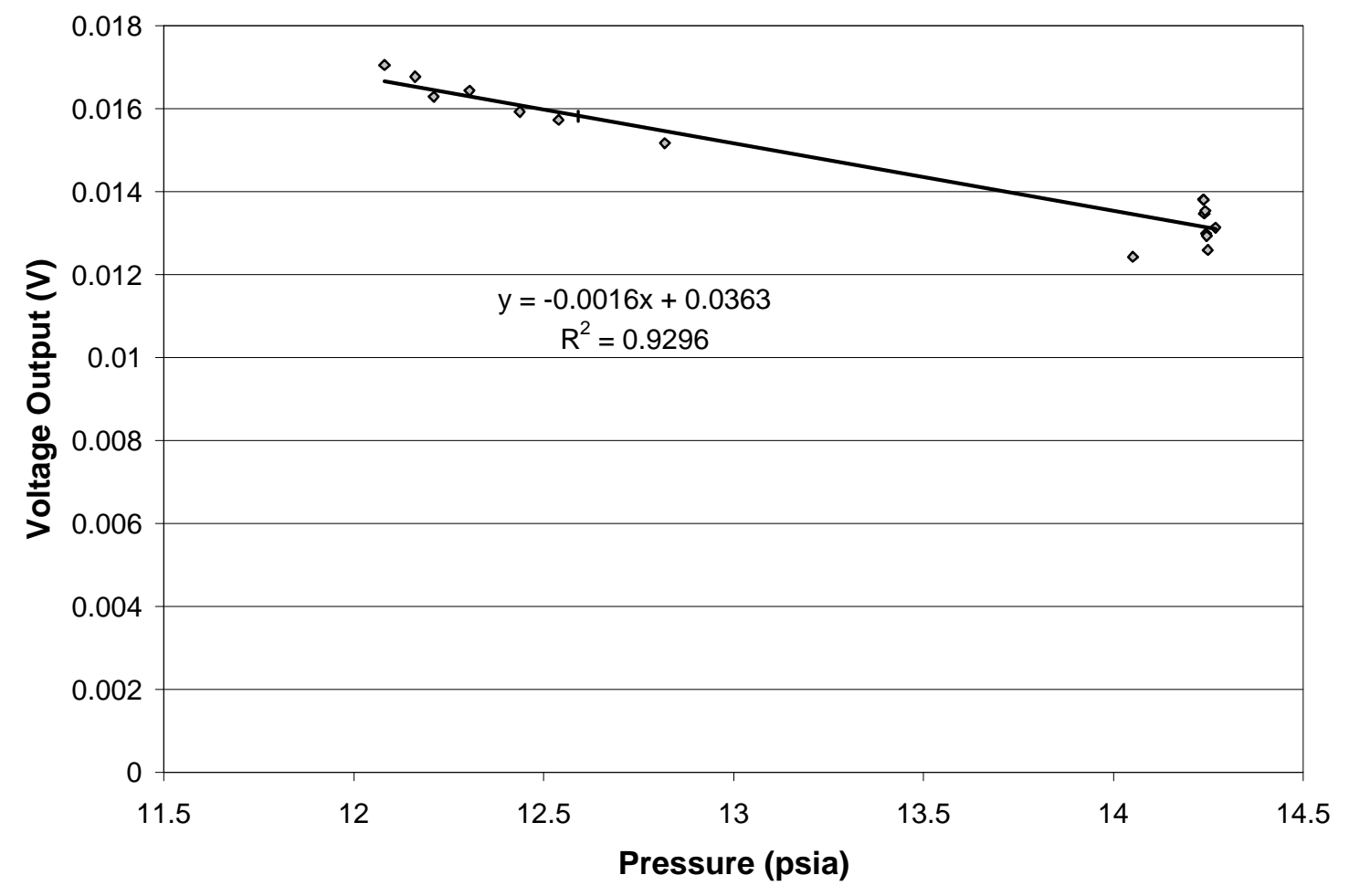

Figure 3.13: Calibration Curve for the Scani-Valve Pressure Transducer.

To better determine the velocity of the airflow along the side of the model a pitotstatic tube was installed. This flow measurement device was placed near the location of maximum thickness of the model and connected to a manometer to determine the velocity of the air. This test was conducted for both the original blockage ratio of $20.8 \%$ and the increased blockage of $21.5 \%$. The blockage ratio was increased by installing 1 inch Styrofoam insulation to the side walls of the test section. Figure 3.14 shows the pitot-static tube installed in the increased blockage test section. 


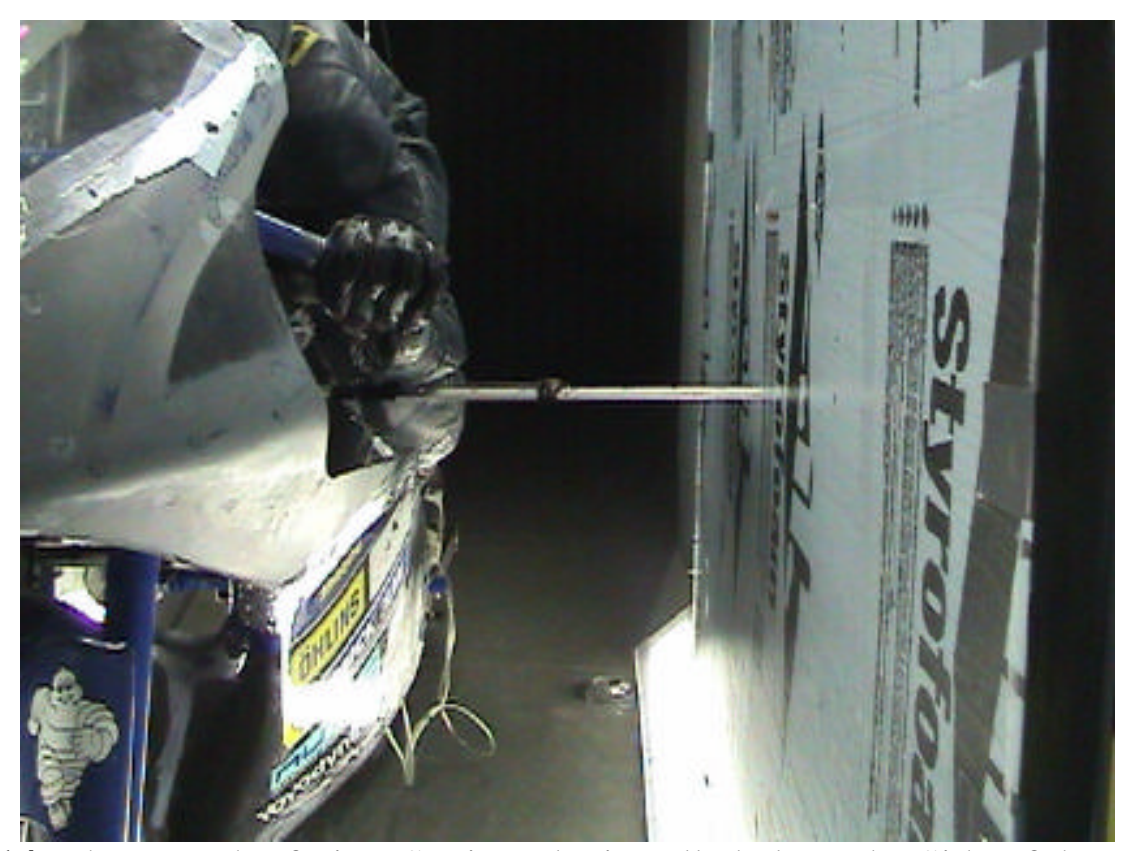

Figure 3.14: Photograph of Pitot-Static Tube installed along the Side of the Model near the Location of Maximum Thickness During the Increased Blockage Test.

\subsection{Testing Apparatus used at Old Dominion University}

With the assistance of Dr. Drew Landman and Masters Degree student Brian Hall from Old Dominion University (ODU), the Tul-Aris model was installed and tested in the Langley Full-Scale Wind Tunnel (LFST). In this test section the blockage ratio was 0.002 , which is well within the accepted standard of a blockage ratio of less than 0.07 according to Barlow, Rae and Pope (1999). The LFST is equipped with an automobile force balance used for the testing of cars. A sting mount was designed and built by Mr. Brian Hall, shown in Figure 3.15, which was installed in the 40ft x $60 \mathrm{ft}$ test section of the LFST. A pressure transducer was also used to measure the surface pressure along the 38 static pressure taps during testing. Existing Labview codes on the LFST control room computers were used for data acquisition. 


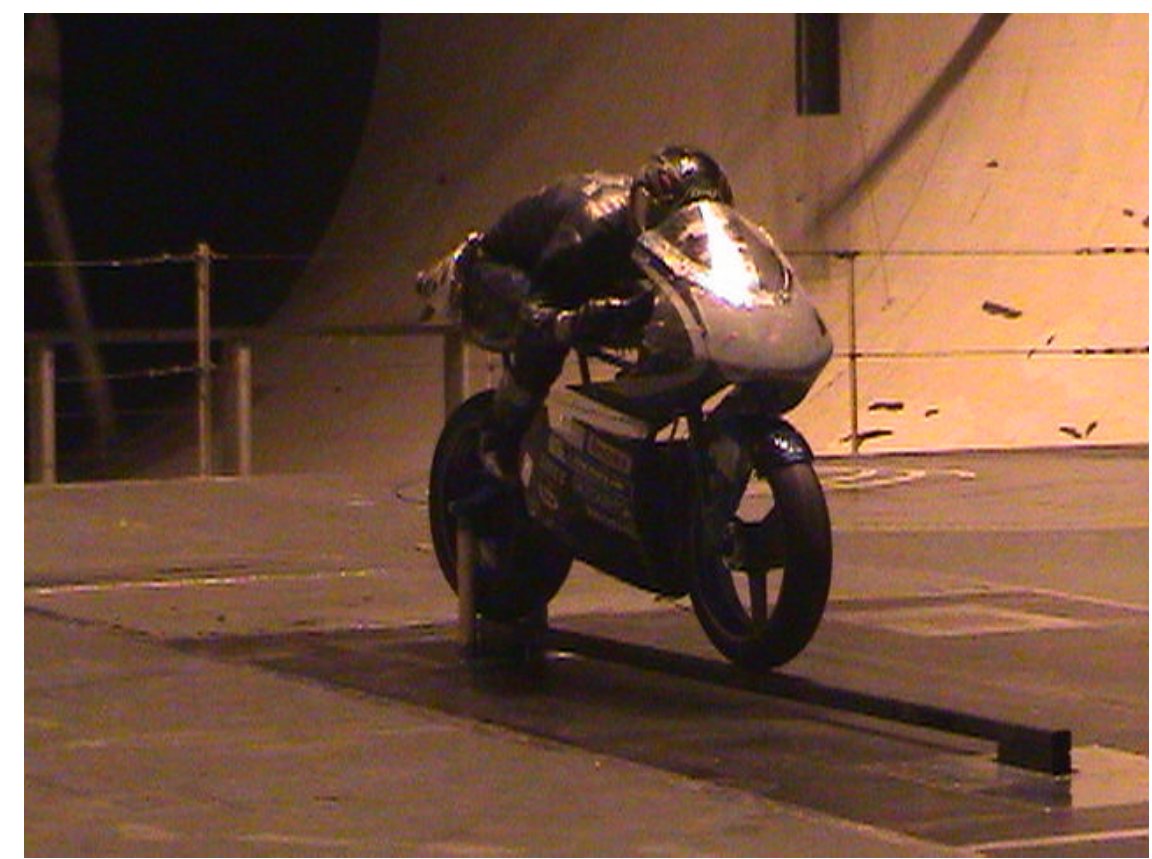

Figure 3.15: Photograph of the Tul-Aris Model and Sting Installed in the Langley Full Scale Tunnel. 


\subsection{Experimental Procedure}

In this chapter the placement of the vortex generators, preliminary testing and the test matrix are described. In addition, wind tunnel operating procedure and data reduction methods are discussed.

\subsection{Vortex Generator Placement}

Due to the lack of experience and empirical knowledge of vortex generators, the placement of these devices is essentially trial and error. To better visualize the location(s) of separation and thus placement of the vortex generator(s), the initial test was a tuft visualization test. Once the locations of flow separation were determined, the vortex generators were gradually added to the model in a symmetrical fashion just upstream of the observed separation point location. The vortex generators were attached to the motorcycle using standard dual temperature hot glue, which can be scraped from the surface.

Knowing the locations in which the flow separated, it was determined that the vortex generator would be placed slightly upstream of the separation. However, this would mean that the configuration depends on the airspeed, since as the airspeed increases the separation location moves forward. It was decided that testing would be done in a gradual process, starting with three VG's, one along the centerline of the bike and a symmetric pair of VG's on the trailing edge of the upper fairing. Additional symmetric pairs of VG's were then added until vortex generators addressed the majority of locations of separated flow. 


\subsection{Preliminary Testing}

Initial testing on the motorcycle was conducted in the WVU Closed Loop Wind Tunnel in a baseline configuration (i.e. vortex generators were not used for this testing). The upper fairing of the Tul-Aris model was modified from its initial shape shown in Figure 4.1 to the shape shown in Figure 4.2. Notice that the changes that were made decrease the angle of incidence of the upper surface of the fairing. The tufts shown in Figure 4.1 were used to determine where the fairing shape should be altered, by identifying areas where flow separation was present. In these locations, the fairing was raised slightly to alleviate this separation region. The trailing edge of the upper surface was extended to increase the air flowing over the rider and to decrease the amount of airflow flowing under the rider. The tufts on the altered fairing experienced smaller fluctuations, thus showing a decrease in the level of turbulence. As a flow separates from a surface the boundary layer height drastically increases, thus resulting in a higher drag force. Any delay in flow separation would effectively decrease the wake and in turn decrease the drag to some degree.

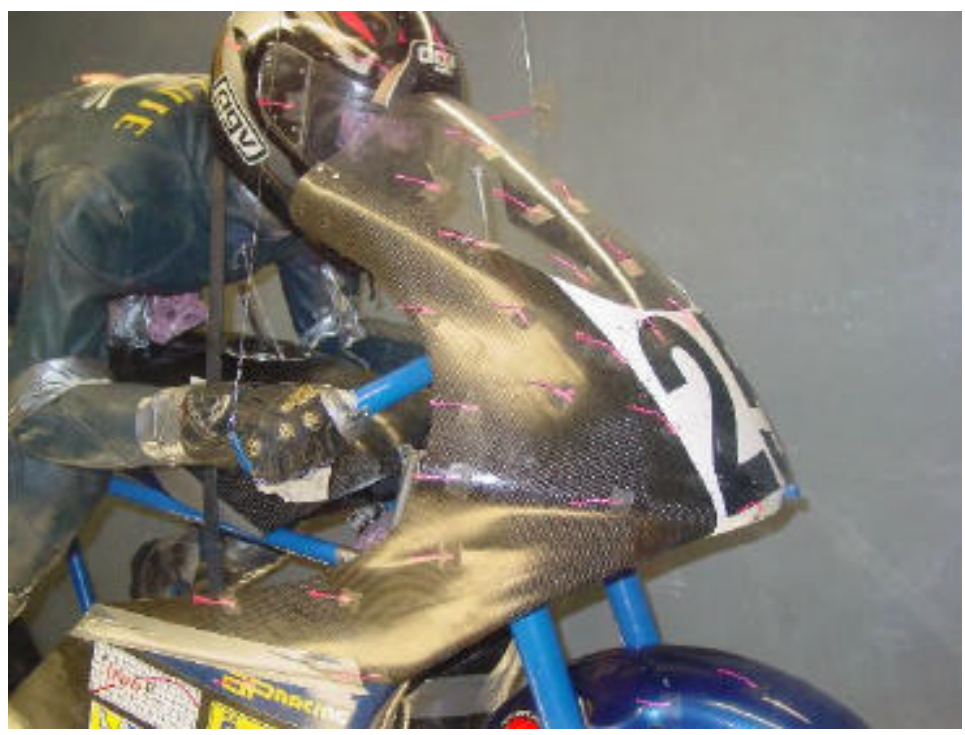

Figure 4.1: Initial Shape of the Upper Fairing of the Tul-Aris Motorcycle. 


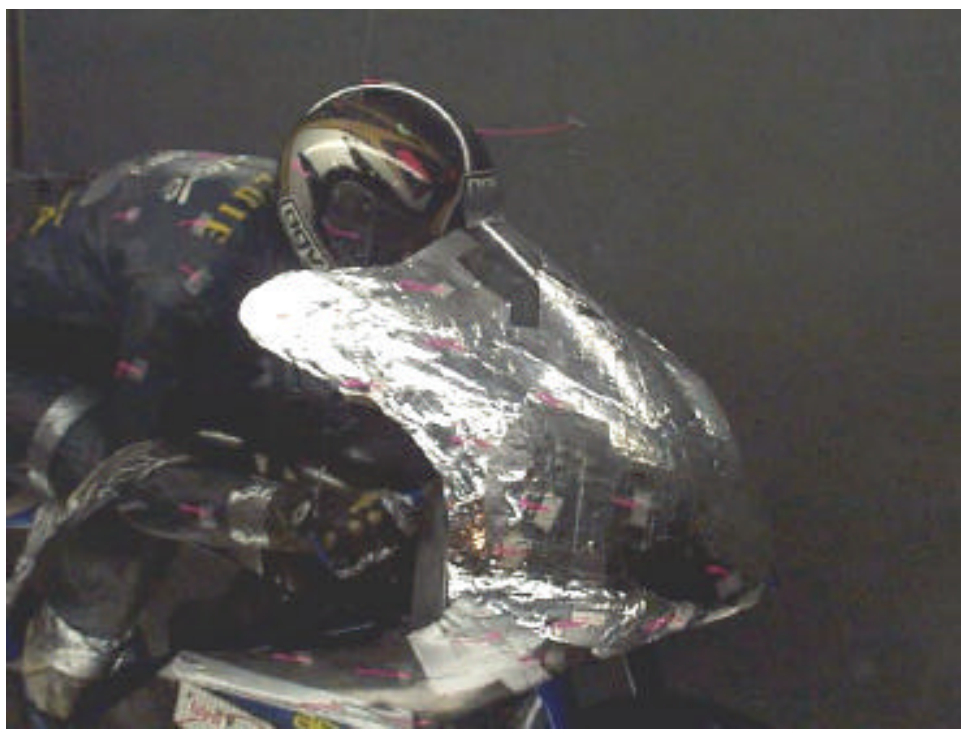

Figure 4.2: Final Shape of the Upper Fairing after Preliminary Testing.

The results of the preliminary testing were implemented in the manufacturing of the racing fairing used in the 2002 race season. From testing at Daytona International Speedway, an increase in the top speed of the motorcycle of $4 \mathrm{mph}$ was noticed over the 2001 version of the Tul-Aris. Since other changes were made to the bike from the 2001 motorcycle, the increase in performance is not completely due to the changes made on the upper fairing in this preliminary testing. Results from the 2002 racing season are two 1st places, five 2 nd places, two 3 rd places, a 4th place, a 7 th place and a lap record at Blackhawk Farms Raceway, Illinois.

\subsection{Test Matrix}

Due to time constraints, it was determined that five different vortex generator configurations were to be tested in Phase I, each of which would add a pair(s) of symmetric VG's to the previous test. Testing was also completed for VG heights of 1/8", $1 / 4$ " and $1 / 2$ ". The progression of tests started with the baseline configuration, with no 
vortex generators on the motorcycle. After the first series of baseline tests, the first vortex generator configuration was added to the helmet of the rider and a pair of VG's on the upper fairing, Configuration 1. The next configuration adds a pair of VG's on the upper fairing to interact with the airflow over the handlebars of the motorcycle, Configuration 2 .

Configurations 3 and 4 both add VG's to Configuration 2. Configuration 3 adds two pairs of VG's at the trailing edge of the lower fairing, while Configuration 4 places these two pairs of vortex generators just upstream of the location of maximum thickness of the lower fairing. Each of these configurations was tested 3 times to produce an average value for comparison with the baseline results. Configuration 5 adds a pair of vortex generators to the lower fairing along the maximum thickness, 3 pairs of VG's to the leading edge of the lower fairing and 2 pairs to the upper fairing along the trailing edge so they are spaced 4 inches apart. After completing tests on the first vortex generator height, the baseline tests were repeated. Then the same vortex generator configurations were tested with the second VG height, followed by another series of baseline tests. Then the final counter-rotating VG height was tested.

After completing the tests with the counter-rotating vortex generators another set of baseline tests were performed. Five modified vortex generator configurations were then tested. The first modified run moved the pair of VG's located near the front wheel to the ends of the handlebar. Modification 2 moved the vortex generators on the upper fairing forward 1 inch. Next, the forward three pairs of VG's on the lower fairing were moved back an inch. The fourth modified configuration moved the VG's nearest the riders' shoulders toward the centerline of the motorcycle by a distance of one inch. The 
fifth and last modified configuration moved the vortex generators forward an additional inch. Table 4.1 reiterates the order in which tests were conducted. Vortex Generators 1 , 2 , and 3 are the VG's with heights of $1 / 2$ ", 1/4", and 1/8" respectively.

Table 4.1: Test Matrix for Tul-Aris Wind Tunnel Testing at WVU, Phase I.

\begin{tabular}{|l|c|c|}
\hline Test Name (Nomenclature) & \# of Tests & Total Tests \\
\hline Baseline 1 (B1) & 3 & 3 \\
\hline Vortex Generator 1, Configuration 1 (VG1C1) & 3 & 6 \\
\hline Vortex Generator 1, Configuration 2 (VG1C2) & 3 & 9 \\
\hline Vortex Generator 1, Configuration 3 (VG1C3) & 3 & 12 \\
\hline Vortex Generator 1, Configuration 4 (VG1C4) & 3 & 15 \\
\hline Vortex Generator 1, Configuration 5 (VG1C5) & 3 & 18 \\
\hline Baseline 2 (B2) & 3 & 21 \\
\hline Vortex Generator 2, Configuration 1 (VG2C1) & 3 & 24 \\
\hline Vortex Generator 2, Configuration 2 (VG2C2) & 3 & 27 \\
\hline Vortex Generator 2, Configuration 3 (VG2C3) & 3 & 30 \\
\hline Vortex Generator 2, Configuration 4 (VG2C4) & 3 & 33 \\
\hline Vortex Generator 2, Configuration 5 (VG2C5) & 3 & 36 \\
\hline Baseline 3 (B3) & 3 & 39 \\
\hline Vortex Generator 3, Configuration 1 (VG3C1) & 3 & 42 \\
\hline Vortex Generator 3, Configuration 2 (VG3C2) & 3 & 45 \\
\hline Vortex Generator 3, Configuration 3 (VG3C3) & 3 & 48 \\
\hline Vortex Generator 3, Configuration 4 (VG3C4) & 3 & 51 \\
\hline Vortex Generator 3, Configuration 5 (VG3C5) & 3 & 54 \\
\hline Baseline 4 (B4) & 3 & 57 \\
\hline
\end{tabular}

Phase II of testing was conducted at the Langley Full Scale Wind Tunnel, repeating Configurations 4 and 5 for all three vortex generator sizes from Phase I. These tests are referred to as runs 2. through 7 . In addition to these tests, two additional configurations were tested. The first of these had the 1/2-inch VG placed at three-inch increments around the rear fairing immediately following the seat (run 9.01). Run 10.01 adds seven pairs of the $1 / 8$-inch $\mathrm{VG}^{\prime}$ 's to run 9.01 on the leading edge of the lower fairing. Table 4.2 lists the tests conducted in the LFST with a description of the VG 
configuration for each test. Tests 1 . and 8 . are the baseline runs. Testing in the LFST was conducted at airspeeds of $55 \mathrm{ft} / \mathrm{s}, 70 \mathrm{ft} / \mathrm{s}$ and $120 \mathrm{ft} / \mathrm{s}$.

After reviewing the results from Phases I and II, the best two configurations from each vortex generator height were retested during Phase III. The purpose of Phase III is to obtain enough data to have an adequate statistical study of the drag coefficient. To have enough data for a statistical study, it was suggested to repeat tests a minimum of 11 times, so it was determined that 2 configurations for each VG would be tested a total of 12 times. To investigate repeatability, the 12 tests were conducted in series of 4 , as shown in Table 4.3.

Table 4.2: Test Matrix for Phase II of Testing in the Langley Full Scale Wind Tunnel.

\begin{tabular}{|c|c|c|c|}
\hline Test & Description & Test & Description \\
\hline 1.01 & Baseline- $55 \mathrm{ft} / \mathrm{s}$ & 6.02 & 1/8-inch VG WVU Config. 4-70 ft/s \\
\hline 1.02 & Baseline-55 ft/s & 6.03 & 1/8-inch VG WVU Config. 4-120 ft/s \\
\hline 1.03 & Baseline- $70 \mathrm{ft} / \mathrm{s}$ & 7.01 & 1/8-inch VG WVU Config. 5-55 ft/s \\
\hline 1.04 & Baseline-70 ft/s & 7.02 & 1/8-inch VG WVU Config. 5-70 ft/s \\
\hline 1.05 & Baseline-120 ft/s & 7.03 & 1/8-inch VG WVU Config. 5-120 ft/s \\
\hline 2.01 & 1/2-inch VG WVU Config. 4-55 ft/s & 8.01 & Baseline2-55 ft/s \\
\hline 2.02 & 1/2-inch VG WVU Config. 4-70 ft/s & 8.02 & Baseline2-70 ft/s \\
\hline 2.03 & 1/2-inch VG WVU Config. 4-120 ft/s & 8.03 & Baseline2-70 ft/s \\
\hline 3.01 & 1/2-inch VG WVU Config. 5-55 ft/s & 8.04 & Baseline2-70 ft/s \\
\hline 3.02 & 1/2-inch VG WVU Config. 5-70 ft/s & 8.05 & Baseline2-120 ft/s \\
\hline 3.03 & 1/2-inch VG WVU Config. 5-120 ft/s & 8.06 & Baseline2-120 ft/s \\
\hline 4.01 & 1/4-inch VG WVU Config. 4-55 ft/s & 9.01 & 1/2-inch VG on Rear Fairing \\
\hline 4.02 & 1/4-inch VG WVU Config. 4-70 ft/s & 10.01 & 1/8-inch VG on Lower Fairing \\
\hline 4.03 & 1/4-inch VG WVU Config. 4-120 ft/s & 11.01 & Drag Test, drag producing device added \\
\hline 5.01 & 1/4-inch VG WVU Config. 5-55 ft/s & 12.01 & Drag Tare of Sting-55 ft/s \\
\hline 5.02 & 1/4-inch VG WVU Config. 5-70 ft/s & 12.02 & Drag Tare of Sting-70 ft/s \\
\hline 5.03 & 1/4-inch VG WVU Config. 5-120 ft/s & 12.03 & Drag Tare of Sting-120 ft/s \\
\hline 6.01 & 1/8-inch VG WVU Config. 4-55 ft/s & & \\
\hline
\end{tabular}


Table 4.3: Test Matrix for Phase III of Tests, Conducted at WVU.

\begin{tabular}{|l|l|l|l|}
\hline Test & Description & Test & Description \\
\hline OB1 & Baseline 1 and 2 & OVG3M1 & 1/8-inch VG Configuration M1 Tests 9-12 \\
\hline OVG1C1 & 1/2-inch VG Configuration 1 Tests 1-4 & OVG3C5 & 1/8-inch VG Configuration 5 Tests 9-12 \\
\hline OVG1C2 & 1/2-inch VG Configuration 2 Tests 1-4 & OVG3C4 & 1/8-inch VG Configuration 4 Tests 9-12 \\
\hline OB1 & Baseline 3 and 4 & OVG2C4 & 1/4-inch VG Configuration 4 Tests 9-12 \\
\hline OVG2C3 & 1/4-inch VG Configuration 3 Tests 1-4 & OVG3P1 & Upper Parametric Position 1 Tests 5-8 \\
\hline OVG2C4 & 1/4-inch VG Configuration 4 Tests 1-4 & OVG3P2 & Upper Parametric Position 2 Tests 5-8 \\
\hline OVG3C4 & 1/8-inch VG Configuration 4 Tests 1-4 & OVG3P3 & Upper Parametric Position 3 Tests 5-8 \\
\hline OVG3C5 & 1/8-inch VG Configuration 5 Tests 1-4 & OVG3P4 & Upper Parametric Position 4 Tests 5-8 \\
\hline OVG3M1 & 1/8-inch VG Configuration M1 Tests 1- & OVG3P4 & Upper Parametric Position 4 Tests 9-12 \\
\hline OVG3P1 & Upper Parametric Position 1 Tests 1-4 & OVG3P3 & Upper Parametric Position 3 Tests 9-12 \\
\hline OVG3P2 & Upper Parametric Position 2 Tests 1-4 & OVG3P2 & Upper Parametric Position 2 Tests 9-12 \\
\hline OVG3P3 & Upper Parametric Position 3 Tests 1-4 & OVG3P1 & Upper Parametric Position 1 Tests 9-12 \\
\hline OVG3P4 & Upper Parametric Position 4 Tests 1-4 & OB1 & Baseline Tests 9 through 16 \\
\hline OVG1C1 & 1/2-inch VG Configuration 1 Tests 5-8 & TSARSIDE & Side Velocity of Increased Blocked \\
\hline OVG1C2 & 1/2-inch VG Configuration 2 Tests 5-8 & TSARB & Increased Blockage Baseline Tests 1-4 \\
\hline OB1 & Baseline Tests 5 through 8 & TSARVG1C2 & Increased Blockage VG1C2 Tests 1-4 \\
\hline OVG2C4 & 1/4-inch VG Configuration 4 Tests 5-8 & TSARVG3P3 & Increased Blockage VG3P3 Tests 1-4 \\
\hline OVG3C4 & 1/8-inch VG Configuration 4 Tests 5-8 & OSIDE & Side Velocity Test \\
\hline OVG3C5 & 1/8-inch VG Configuration 5 Tests 5-8 & OB1 & Baseline Tests 17 through 20 \\
\hline OVG3M1 & 1/8-inch VG Configuration M1 Tests 5- & LFPT & Lower Fairing Parametric Tests \\
\hline
\end{tabular}

After the completion of Phase III five additional tests were run. The first of these was to obtain a better estimation of the airspeed along the side of the motorcycle, which was accelerated due to wind tunnel blockage. This was measured using an additional pitot-static probe to the system along the location of maximum thickness of the lower fairing of the motorcycle. A better understanding of the effects of blockage was investigated in the final four tests. The test section cross-sectional area was reduced by adding 1-inch home insulation foam to the sides of the test section, reducing the area by $1.03 \mathrm{ft}^{2}$. With this increased blockage three configurations were tested: baseline, VG3P3 and VG1C2. Additionally the air velocity along the side of the increased blockage tests was measured using the pitot-static probe. 


\subsection{Wind Tunnel Testing Procedure}

After determining how many and where to place the vortex generators it was time to run the wind tunnel. Once the motorcycle model was mounted into the wind tunnel and the proper vortex generator array added to the model the tunnel was run.

The start up procedure for the WVU Closed Loop Wind Tunnel is as follows:

- Close Main Breaker

- Turn On Propeller Circuit

- Press Motor Generator Set Start Button

- Press Line Contactor Close Button

- Slowly Increase Motor Speed

- Increase Propeller Pitch to Obtain Desired Tunnel Velocity

After allowing tunnel transients to level off, pressure, temperature and drag force data were collected though the computerized data acquisition system. Tests were completed for each configuration in the test matrix; these tests were conducted without altering the controls for the wind tunnel. After one series of tests were completed the tunnel was shut down using the following procedure:

- Decrease Propeller Pitch to Reach Zero Velocity

- Slowly Decrease Motor Speed

- Press Line Contactor Open Button

- Press Motor Generator Set Stop Button

- Turn Propeller Circuit Off

- Open Main Circuit Breaker 
With the tunnel shut down, the vortex generator configuration was changed while the model was still installed in the test section. Once the alterations were made to the model, the tunnel was restarted and testing conducted as stated above.

\subsection{Data Reduction}

The first calculation that was performed in the course of this experimentation was to determine the blockage ratio of the test section. Using Figure 3.9 the frontal area of the motorcycle was determined. This was used in conjunction with the known dimensions of the test section (73.250 inches by 50.375 inches) to determine the blockage ratio through Equation 4.1. In this relationship $B R$ is the test section blockage ratio, $A$ is the model frontal area and $S$ is the test section cross-sectional area.

$$
B R=\frac{A}{S}
$$

The airspeed in the test section of the $4 \mathrm{ft}$ by $6 \mathrm{ft}$ test section was determined through a modified Bernoulli equation, shown in Equation 4.2 where the manometer was connected to a pitot-static probe. Here, $V$ is the velocity in $\mathrm{ft} / \mathrm{s}, g$ is the gravitational acceleration, $\rho_{\text {liquid }}$ is the density of the manometer liquid, $h$ is the height of the manometer and $\rho_{\text {air }}$ is the air density.

$$
V(f t / s)=\sqrt{\frac{2 g \rho_{\text {liquid }} h}{\rho_{\text {air }}}}
$$

Using the relationships determined during calibration of the load cell and pressure transducer the voltages recorded by the data acquisition system where converted to their corresponding force and pressure values. Once the signals were converted from voltage the mean values were calculated using Equation 4.3. Here, $X(t)$ is the recorded signal, $\bar{X}$ 
is the mean value of the signal, $t_{o}$ is the start time of the test, $\Delta t$ represents the time step between data points.

$$
\bar{X}=\frac{\int_{t_{o}}^{t_{o}+\Delta t} X(t) d t}{\Delta t}
$$

To calculate the drag force measured during Phase I of testing the velocity was adjusted for the reduced area caused by the test section blockage. Equation 4.4 was used to adjust the velocity, where $V_{a c t}$ is the actual velocity along the sides of the model, $V_{\text {meas }}$ is the measured free-stream velocity, $A_{T . S .}$ is the test section cross-sectional area and $A_{f r}$ is the model frontal area.

$$
V_{\text {act }}=V_{\text {meas }} \frac{A_{T . S .}}{\left(A_{\text {T.S. }}-A_{f r}\right)}
$$

After completion of the side velocity measurements the actual velocity of the air was recalculated using the ratio of the pitot-static measurements, along the side of the model and upstream of the model.

The pressure in the large test section was measured to be 0.065 psig. The corresponding absolute pressure was used to determine the density of the air in the test section through the Ideal Gas Law, Equation 4.9. Here, $\rho$ is the density, $P$ is the local pressure as determined through Equation 4.7, $R$ is the gas constant (for air equal to 1716 $\left.\mathrm{ft}-\mathrm{lb} / \mathrm{slug}-{ }^{\circ} \mathrm{R}\right)$, and $T$ is the average local air temperature as measured from the thermocouple during the respective test.

$$
\rho=\frac{P}{R T}
$$

Now that the properties of the airflow were known the forces on the sting could be calculated. To do this the Reynolds number based on the diameter of the support 
cables, D, was determined, from Equation 4.10. Similarly the Reynolds Number needed to be determined based on the length of the sting support arm, where the D represents this length, exposed to the free stream flow.

$$
\operatorname{Re}=\frac{\rho U D}{\mu}
$$

Assuming the support cables behave as smooth cylinders, their drag coefficient according to Young, Munson and Okiishi (1997) was 1.2. The drag force on the cables was determined from Equation 4.11, where $D_{\text {cable }}$ is the drag force, $\rho$ is the density of air, $V$ is the air velocity, $L$ is the total length of exposed cable, $D$ is the diameter of the cable and $C_{D}$ is the drag coefficient.

$$
D_{\text {cables }}=1 / 2 C_{D} \rho V^{2} L D
$$

The drag on the exposed part of the sting was broken down into two components: the flat plate parallel to the flow direction, and the rectangle perpendicular to the flow. Equation 4.12 was used to determine the drag coefficient on the flat plate portion of the sting, and used in Equation 4.13 to determine the drag force on this part of the sting. $C_{D f}$ is the coefficient of drag on the flat plate, $R e_{L}$ is the Reynolds Number based on the length of the plate $\left(4.5 \times 10^{5}\right), D_{F . P .}$ is the drag force on the flat plate, $L$ is the length of the flat plate (15.25 in), $W$ is the width of the component ( 2 in), $\rho$ and $V$ are the density and velocity of the air, respectively.

$$
\begin{gathered}
C_{D f}=\frac{1.328}{\sqrt{\operatorname{Re}_{L}}} \\
D_{F . P .}=1 / 2 C_{D_{f}} \rho V^{2} L W
\end{gathered}
$$


The rectangular tube that was used for this component of the sting also had a component of drag due to the 1-inch by 2 -inch face of the tube that was perpendicular to the flow. This portion of the sting drag was calculated using Equation 4.14, where $D_{\text {rect }}$ is the drag on the rectangular portion of the sting, $C_{D}$ is the drag coefficient based on the length, $L$, to width, $W$, ratio of the rectangle.

$$
D_{\text {rect }}=1 / 2 C_{D} \rho V^{2} L W
$$

The drag coefficient of the motorcycle was determined through Equation 4.15, where $C_{D m}$ is the drag coefficient of the model, $D_{\text {meas }}$ is the measured drag force, $D_{\text {cable }}$ is the calculated drag on the support cables, $D_{\text {sting }}$ is the force calculated on the sting, $A_{f r}$ is the frontal area of the motorcycle.

$$
C_{D_{m}}=\frac{\left(D_{\text {meas }}-D_{\text {cable }}-D_{\text {sting }}\right)}{1 / 2 \rho V^{2} A_{f r}}
$$

The pressure coefficients, $C_{p}$, were determined through Equation 4.16 where $P_{a t m}$ is the atmospheric pressure, $P_{\text {meas }}$ is the measured pressure.

$$
C_{p}=\frac{P_{\text {meas }}-P_{\text {atm }}}{1 / 2 \rho V^{2}}
$$

The average drag coefficients were calculated for each test configuration for comparison purposes. The average values were then compared to the baseline average in terms of percent difference, \%Diff (Equation 4.17), where $C_{\text {Davg_test }}$ is the average coefficient of drag for a specific test, $C_{\text {Davg_B }_{B}}$ is the baseline average drag coefficient.

$$
\% \text { Diff }=\frac{C_{D_{\text {avg }_{\text {test }}}}-C_{D_{\text {avg }_{-} B}}}{C_{D_{\text {avg }_{-} B}}} x 100
$$


All experimental measurements have some error associated with it, knowing the error in the measurements is important. A propagation of error technique was used to determine the error in the calculated dependent variable and is given in Equation 4.18. In this relationship $W_{r}$ is the uncertainty in the calculation of the result, $R, x_{i}$ is the measured independent variable and $w_{i}$ is the uncertainty of the measured variable.

$$
W_{r}=\left[\left(\frac{\partial R}{\partial x_{1}} w_{1}\right)^{2}+\left(\frac{\partial R}{\partial x_{2}} w_{2}\right)^{2}+\ldots+\left(\frac{\partial R}{\partial x_{n}} w_{n}\right)^{2}\right]^{1 / 2}
$$

Appling the above relationship to the drag coefficient, $C_{D}$, Equation 4.19 results. There were four measured variables in the determination of the drag coefficient: drag force, $D$, the pressure, $P$, temperature, $T$, and the airspeed, $V$.

$$
\delta C_{D}=\left[\left(\frac{\partial C_{D}}{\partial D} \delta D\right)^{2}+\left(\frac{\partial C_{D}}{\partial P} \delta P\right)^{2}+\left(\frac{\partial C_{D}}{\partial T} \delta T\right)^{2}+\left(\frac{\partial C_{D}}{\partial V} \delta V\right)^{2}\right]^{1 / 2}
$$

Combining the definition of drag coefficient, Equation 4.20, and the Ideal Gas Law, Equation 4.9, the drag coefficient is expressed in terms of the four measured variables as Equation 4.21, where $R^{\prime}$ is the ideal gas constant for air.

$$
\begin{gathered}
C_{D}=\frac{D}{0.5 \rho V^{2} A} \\
C_{D}=\frac{D}{0.5 \frac{P}{R^{\prime} T} V^{2} A}=\frac{2 R^{\prime} D T}{P V^{2} A}
\end{gathered}
$$

From this relationship, the partial derivatives of $C_{D}$ with respect to each of the measured variables (to be used in Equation 4.19) were determined and given below in Equations 4.22 through 4.25. Instrument errors for the measuring devices used in the WVU testing are shown in Table 4.4. 


$$
\begin{gathered}
\frac{\partial C_{D}}{\partial D}=\frac{2 R^{\prime} T}{P V^{2} A} \\
\frac{\partial C_{D}}{\partial P}=\frac{-2 D R^{\prime} T}{P^{2} V^{2} A} \\
\frac{\partial C_{D}}{\partial T}=\frac{2 D R^{\prime}}{P V^{2} A} \\
\frac{\partial C_{D}}{\partial V}=\frac{-4 D R^{\prime} T}{P V^{3} A}
\end{gathered}
$$

Table 4.4: Instrumentation Errors for Measuring Devices

\begin{tabular}{|l|l|}
\hline Instrument & Error \\
\hline Omegadyne load cell & $\pm 0.03 \%$ \\
\hline Scani-Valve Pressure Transducer & $\pm 0.5 \%$ \\
\hline Omega Type-J Thermocouple & $\pm 0.20^{\circ} \mathrm{C}$ \\
\hline
\end{tabular}




\subsection{Results}

In this chapter the experimental results from each phase of testing will be discussed. All drag coefficient changes shown are relative to the average baseline drag coefficient for that particular phase of testing. Pressure coefficient data was calculated based on the measured static pressure.

\subsection{Phase I Results}

Phase I of testing was conducted in the WVU Closed Loop Wind Tunnel. These results were used to reduce the number of configurations tested in Phases II and III. Figure 5.1 shows the average relative change in drag coefficient, from the baseline value of 1.163 , for the 20 configurations tested in Phase I. The error bars displayed in this figure illustrate instrumentation error for the drag coefficient, determined from Equation 4.19 to be \pm 0.007 . The most promising configurations for drag reduction were found to be those with the 1/8-inch vortex generator. These results are also tabulated in Table 5.1, refer to Table 4.1 for descriptions of the test nomenclature, with the relative change in $C_{D}$ $\left(\Delta \mathrm{C}_{\mathrm{D}}\right)$ displayed as well as the standard deviation of the measurement. There is no standard deviation for the last five entries in the table because there was only one test measured with that particular configuration.

The effect of the vortex generator height on drag reduction was determined during Phase I. Figure 5.2 shows the effects of VG height on the drag coefficient for Configuration 1. Similarly, Figures 5.3 through 5.6 show the dependence of the relative drag coefficient on the height of the device for Configurations 2 through 5, respectively. For all configurations it was determined that the general trend was that as the height of 
the VG increases its ability to reduce drag decreases. However, as the height becomes smaller the rate of change in $\Delta C_{D}$ is less, if not reaching a plateau between the 1/4-inch and the 1/8-inch vortex generator as seen for Configurations 3, 4, and 5. It is important to note that this optimum height will change for any change in speed and possibly blockage ratio, since it is dependent upon the boundary layer thickness. It is believed that the effectiveness of the VG is diminished if the height of the device is allowed to protrude through the boundary layer.

Table 5.1: Measured Change in Drag Coefficient and the Standard Deviation of the Measurement for Phase I of Testing.

\begin{tabular}{|c|c|c|}
\hline Test & $\Delta \mathrm{C}_{\mathrm{D}}$ & STDEV \\
\hline BaselineM & -0.014 & 0.0064 \\
\hline Baseline & 0 & 0.0267 \\
\hline VG1C1 & 0.089 & 0.0739 \\
\hline VG1C2 & 0.119 & 0.0883 \\
\hline VG1C3 & 0.355 & 0.0195 \\
\hline VG1C4 & 0.329 & 0.2014 \\
\hline VG1C5 & 0.229 & 0.2793 \\
\hline VG2C1 & 0.003 & 0.0150 \\
\hline VG2C2 & 0.000 & 0.0083 \\
\hline VG2C3 & -0.055 & 0.0242 \\
\hline VG2C4 & -0.050 & 0.0172 \\
\hline VG2C5 & -0.031 & 0.0128 \\
\hline VG3C1 & -0.080 & 0.0219 \\
\hline VG3C2 & -0.067 & 0.0231 \\
\hline VG3C3 & -0.071 & 0.0126 \\
\hline VG3C4 & -0.042 & 0.0065 \\
\hline VG3C5 & -0.052 & 0.0054 \\
\hline VG3M1 & -0.118 & ------ \\
\hline VG3M2 & -0.060 & ----- \\
\hline VG3M3 & -0.035 & ------ \\
\hline VG3M4 & -0.031 & ------ \\
\hline VG3M5 & -0.028 & ------ \\
\hline
\end{tabular}

The modified baseline configuration (BaselineM) was taken after removing the radiator simulation from the model. It is important to note that the modified baseline 
from Phase I is the baseline configuration for Phase II and III. In addition, there was a 14 drag count reduction in the drag coefficient as well as a decrease in the standard deviation of the measurements.

Surface pressure readings were also taken during Phase I of testing. Figure 5.7 shows the pressure coefficients for the first set of baseline tests. Refer to Figure 3.3 for the locations of the pressure taps. The rows of four taps are numbered from top to bottom of the lower fairing starting with Tap 9. The first eight pressure taps are located on the upper fairing starting at the front and increasing in tap number to the trailing edge. It is important to note that there was some variation in the pressure readings between tests as seen from the additional pressure figures shown in Appendix A, with two primary causes to these fluctuations. The first of these is the instrumentation error of the Scani-Valve pressure transducer, and the other source of fluctuation is the unsteady, turbulent nature of the flow over the motorcycle, partially due to the non-uniform horizontal velocity profile caused by the upstream turn in the wind tunnel.

Figure 5.8 shows the pressure coefficients for the 1/2-inch vortex generator test of Configuration 1. Comparing the values of this figure to that of the baseline figure, it is seen that the pressure coefficients for Taps 4 through 7 are less negative than the baseline case. But the rest of the taps experience an increase in negative pressure; which shows there is a net upstream movement of the separation because of the increased negative pressure.

The 1/4-inch vortex generator pressure coefficients for Configuration 2 is shown in Figures 5.9. In this configuration there was a slight decrease in drag coefficient, which is caused by the slight overall increase, in the positive direction, in some of the pressure 
coefficients. Figure 5.10 shows the pressure distribution for the $1 / 8$-inch VG, Configuration 3 test. These pressures are less negative than the baseline configuration which results in the 50 drag count ( 1 drag count is equal to a drag coefficient of 0.001$)$ reduction in the total drag.

After testing these five configurations, modified VG arrangements were tested. The first of these was to place a pair of VG's on the ends of the handlebars. This testing had little effect on the pressure coefficients, shown in Figure 5.11, since there were no taps in the vicinity of the handlebars. However, as shown in Figure 5.1 there was a considerable reduction of 89 drag counts, compared to the baseline.

The pressure coefficients of individual taps were also looked at to determine any trends between the change in pressure at a certain location and any reduction in the drag coefficient. Figure 5.12 displays the pressure coefficients for Tap Number 1 for tests conducted in Phase I on the first five configurations. On the lower fairing the pressure taps were located in rows of four and in series of staggered columns. Tap Number 23 is close to the vertical middle of the lower fairing near the leading edge, therefore it is of interest to look at the pressure coefficients for this tap, Figures 5.13. Again, from these figures no true relationship can be clearly seen by looking merely at one tap location at a time. From looking at the pressure figures, primarily those for each configuration (shown in this section and in Appendix A) the location of separation can be found were the large negative increases in pressure occur. 


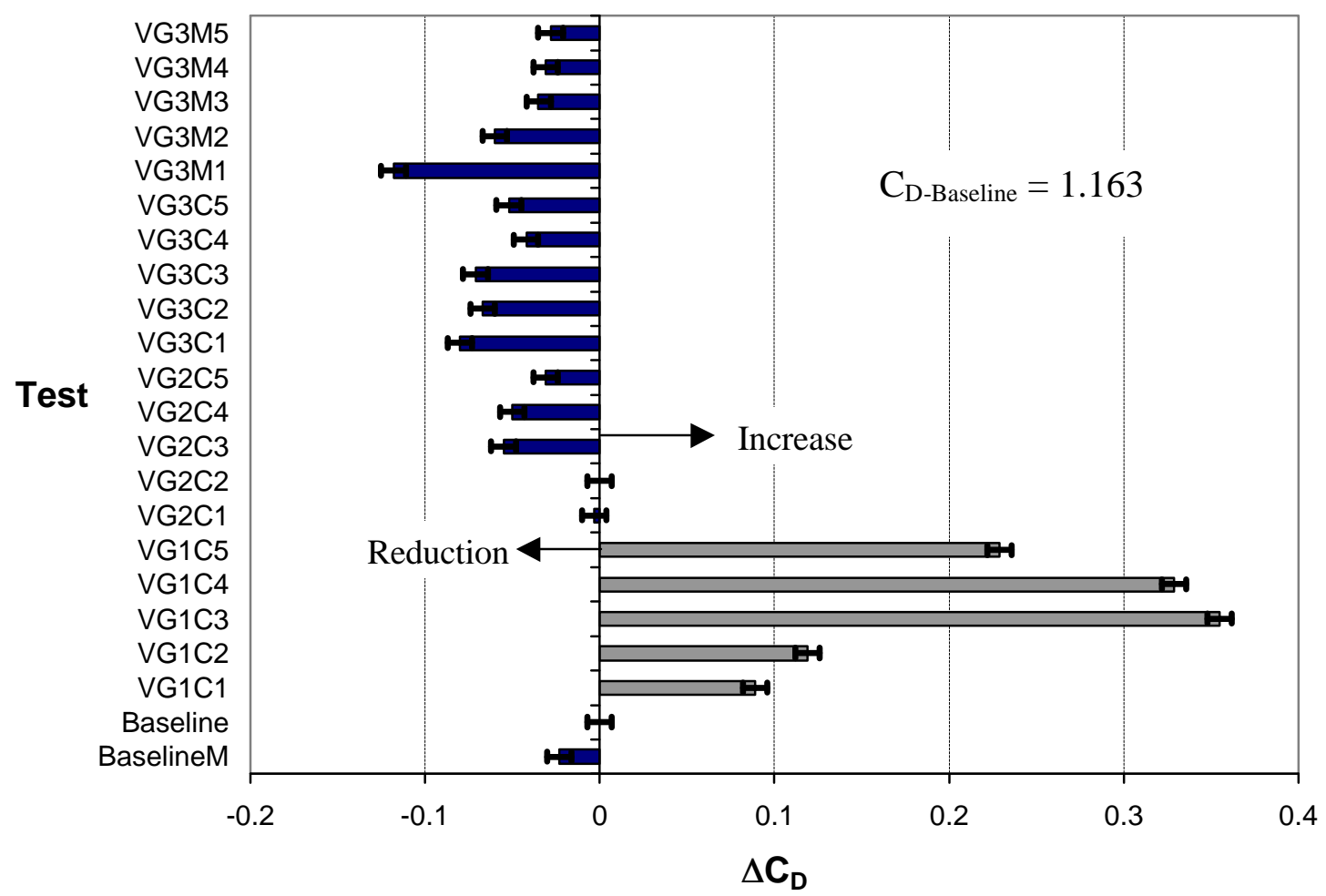

Figure 5.1: Comparison of Relative Drag Coefficients for Phase I of Testing Conducted in the West Virginia University Closed Loop Wind Tunnel.

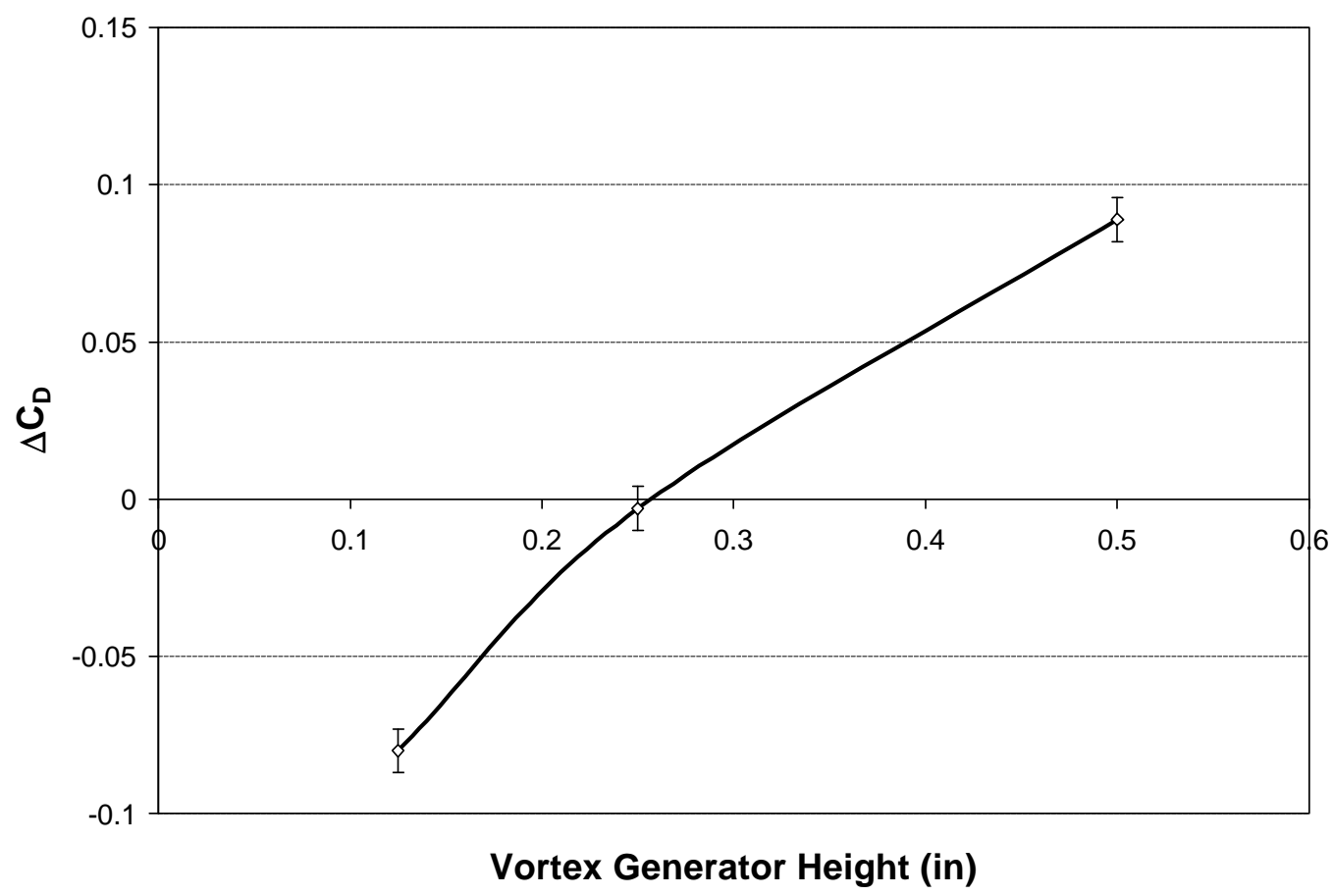

Figure 5.2: Effects of Vortex Generator Height on Configuration 1 


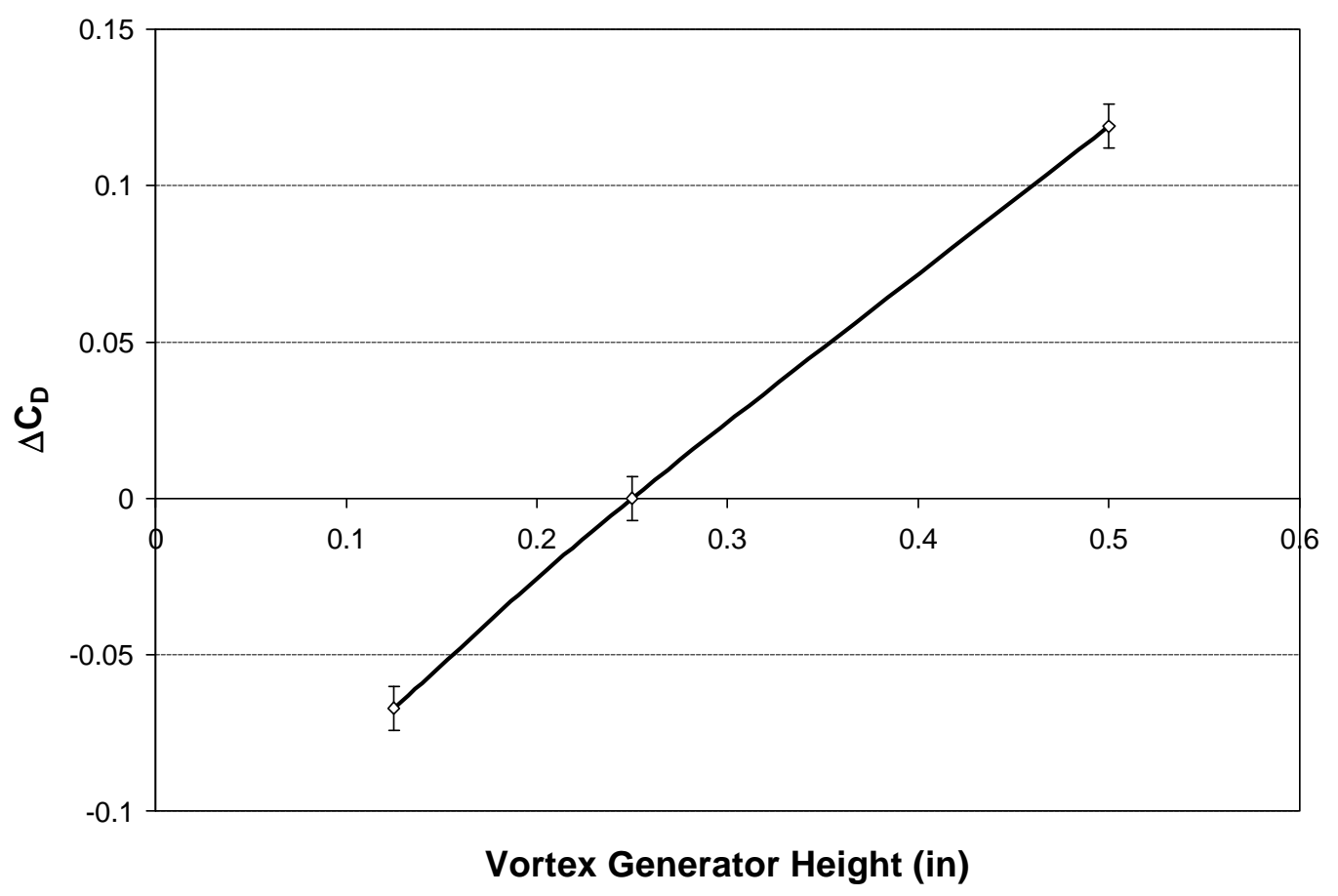

Figure 5.3: Effects of Vortex Generator Height on Configuration 2

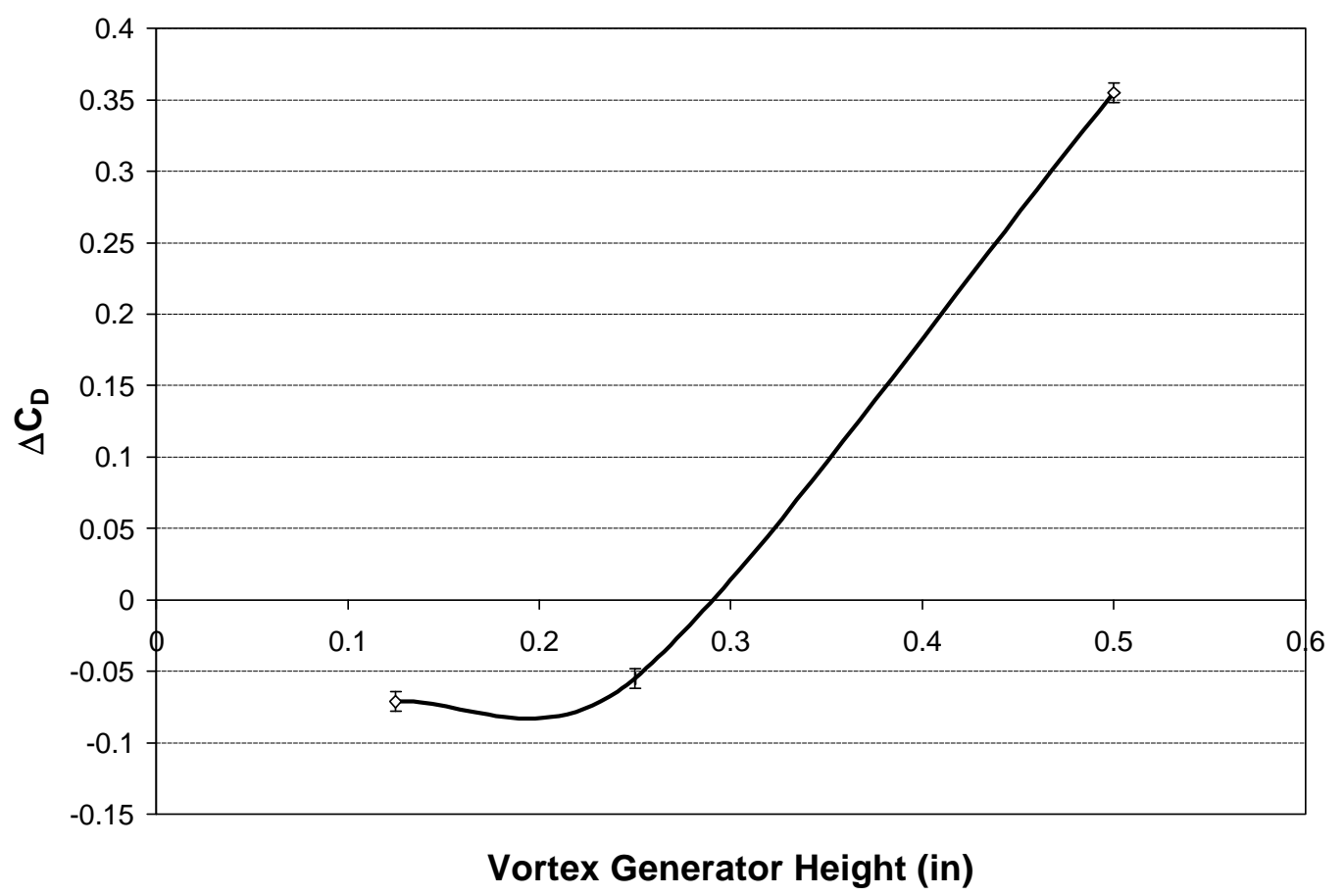

Figure 5.4: Effects of Vortex Generator Height on Configuration 3 


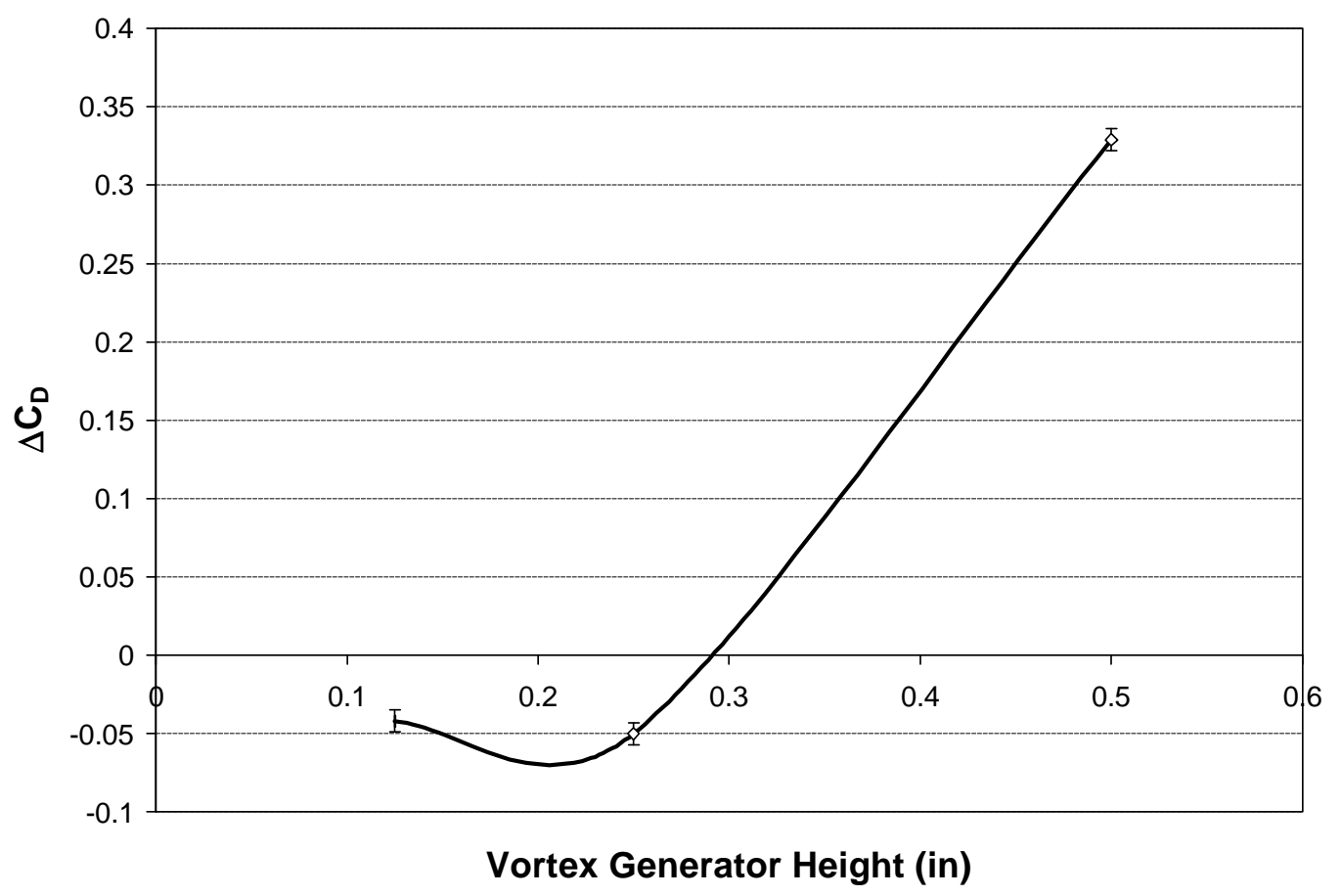

Figure 5.5: Effects of Vortex Generator Height on Configuration 4

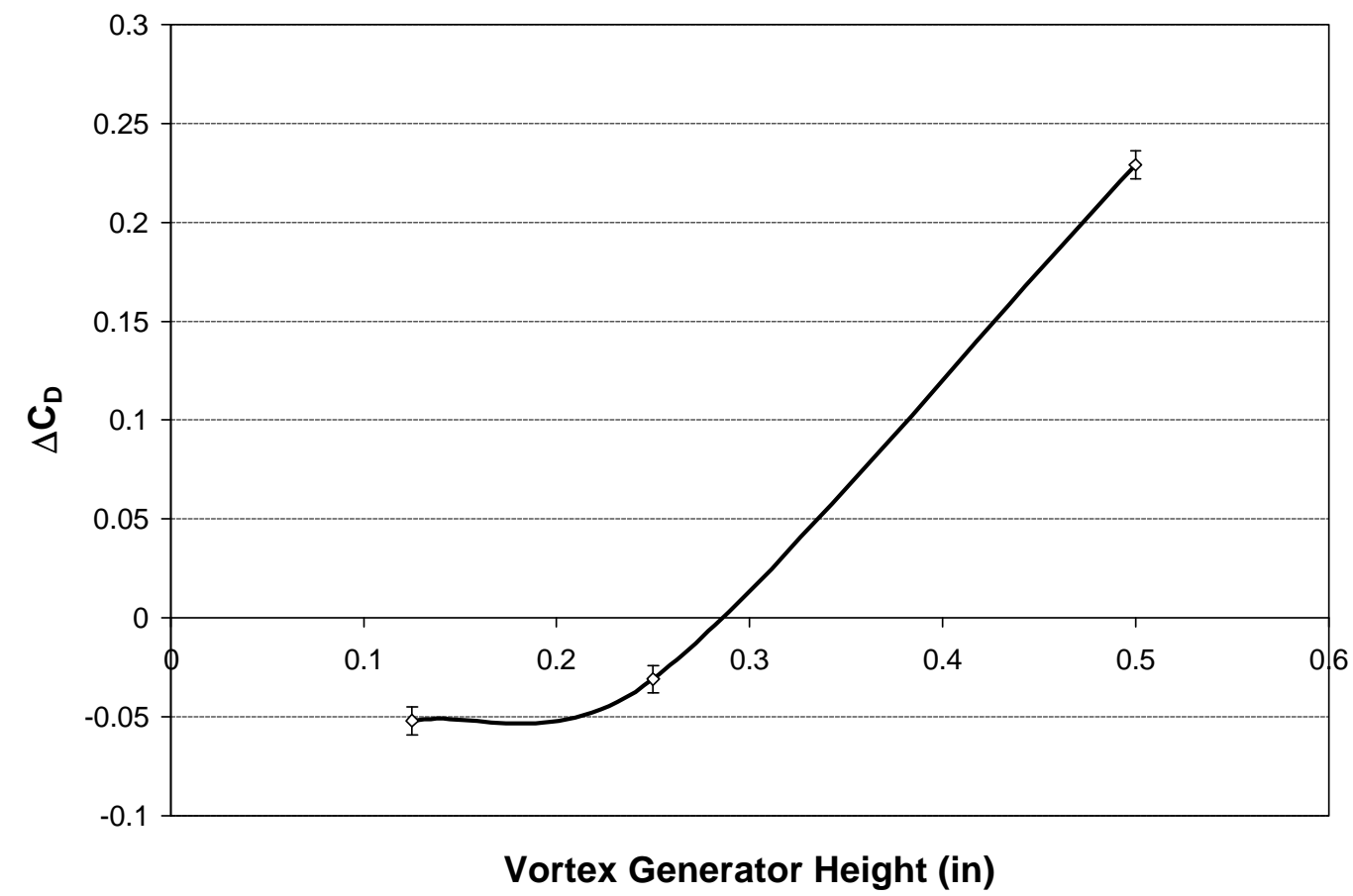

Figure 5.6: Effects of Vortex Generator Height on Configuration 5 


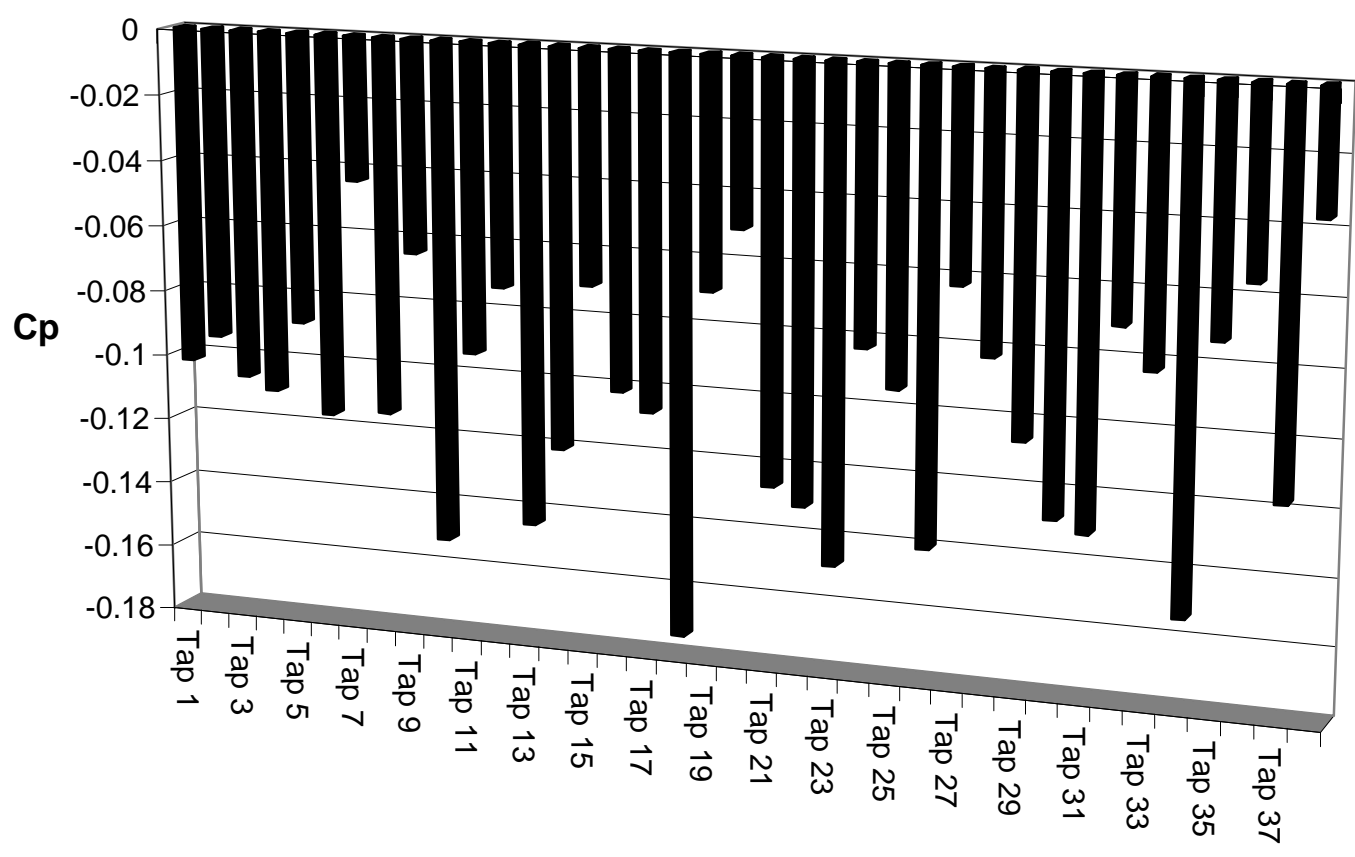

Figure 5.7: Phase I Average Pressure Coefficients for Three Baseline 1 Tests.

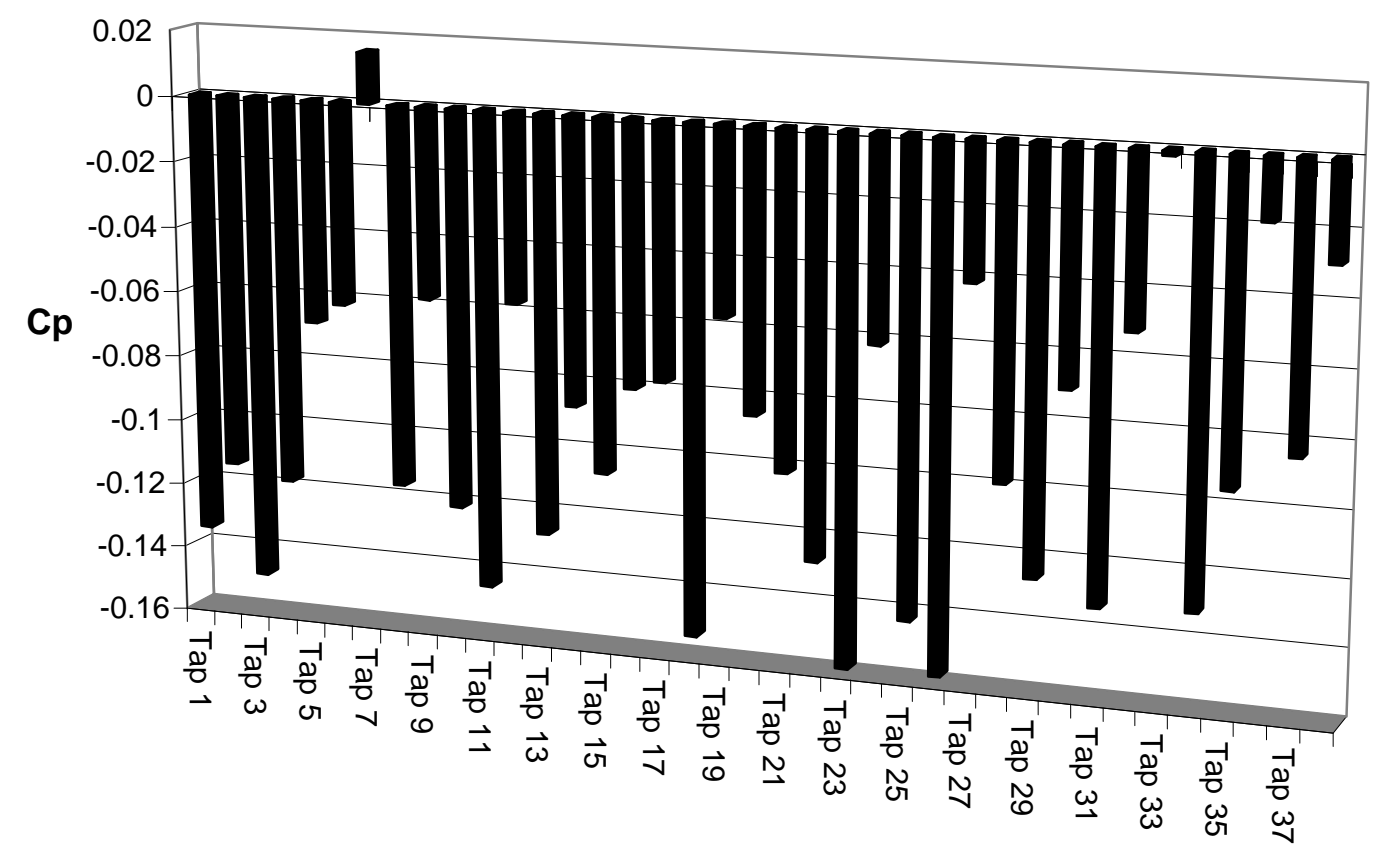

Figure 5.8: Phase I Average Pressure Coefficients for Three 1/2-inch VG Configuration 1 Tests. 


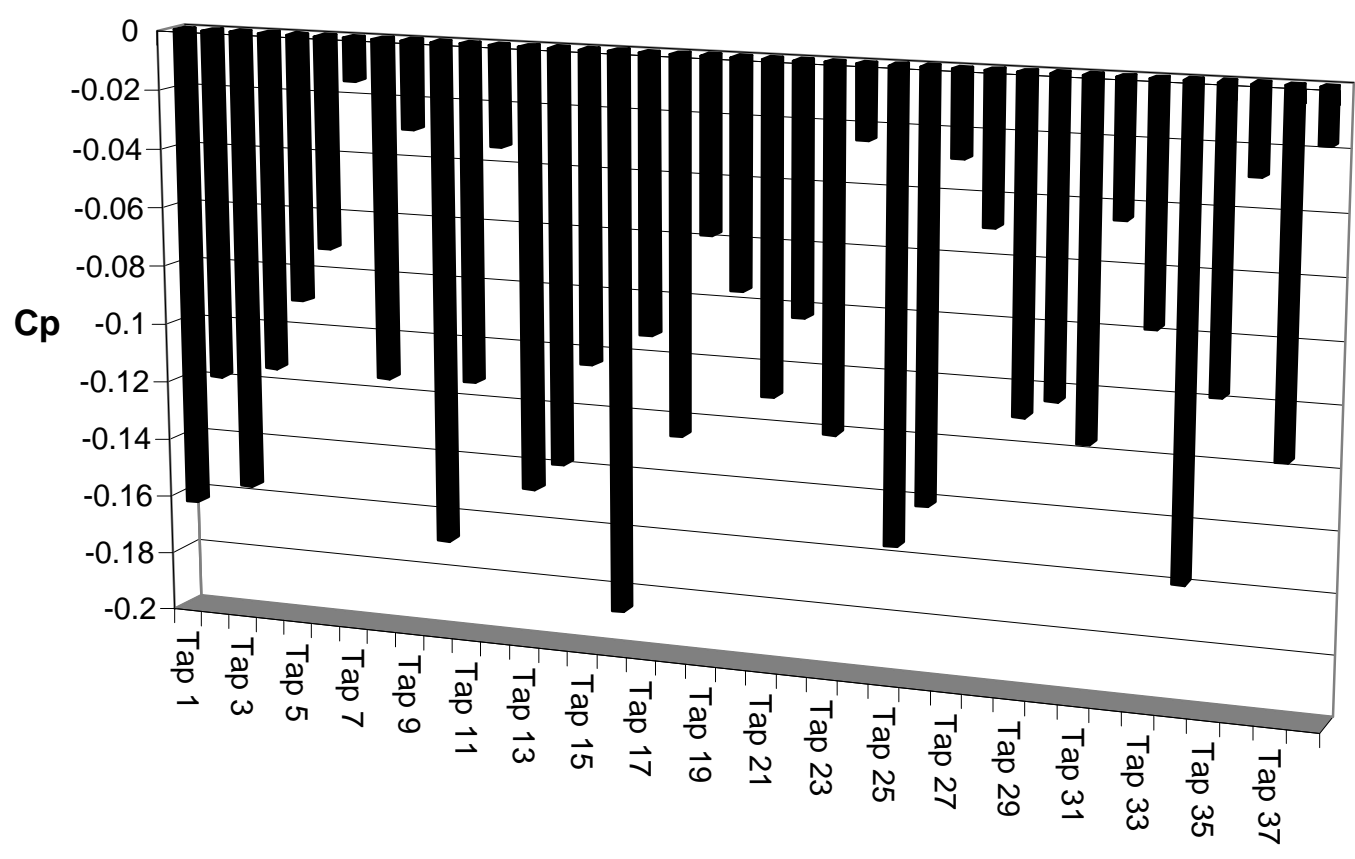

Figure 5.9: Phase I Average Pressure Coefficients for Three 1/4-inch VG Configuration 2 Tests.

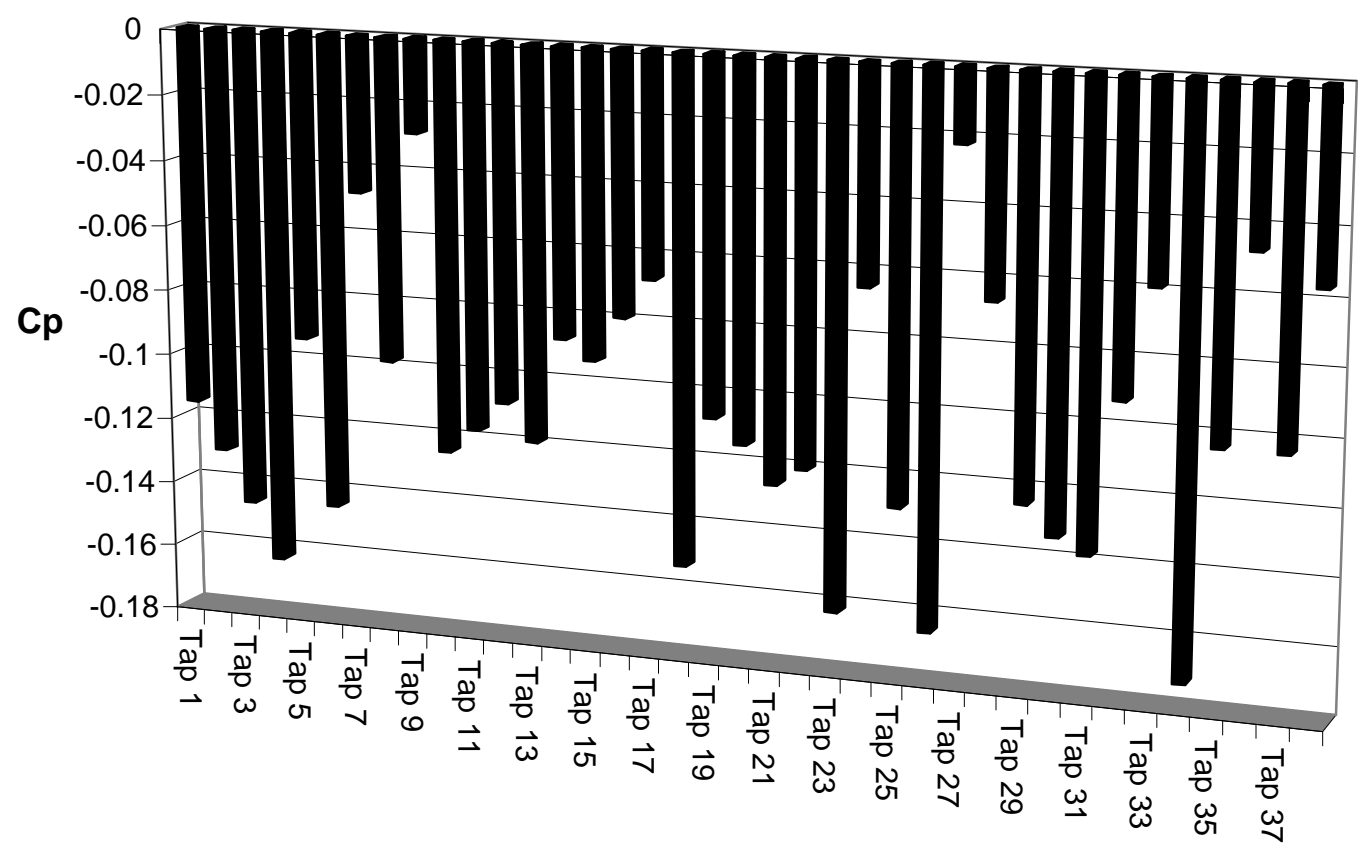

Figure 5.10: Phase I Average Pressure Coefficients for Three $1 / 8$-inch VG Configuration 3 Tests. 


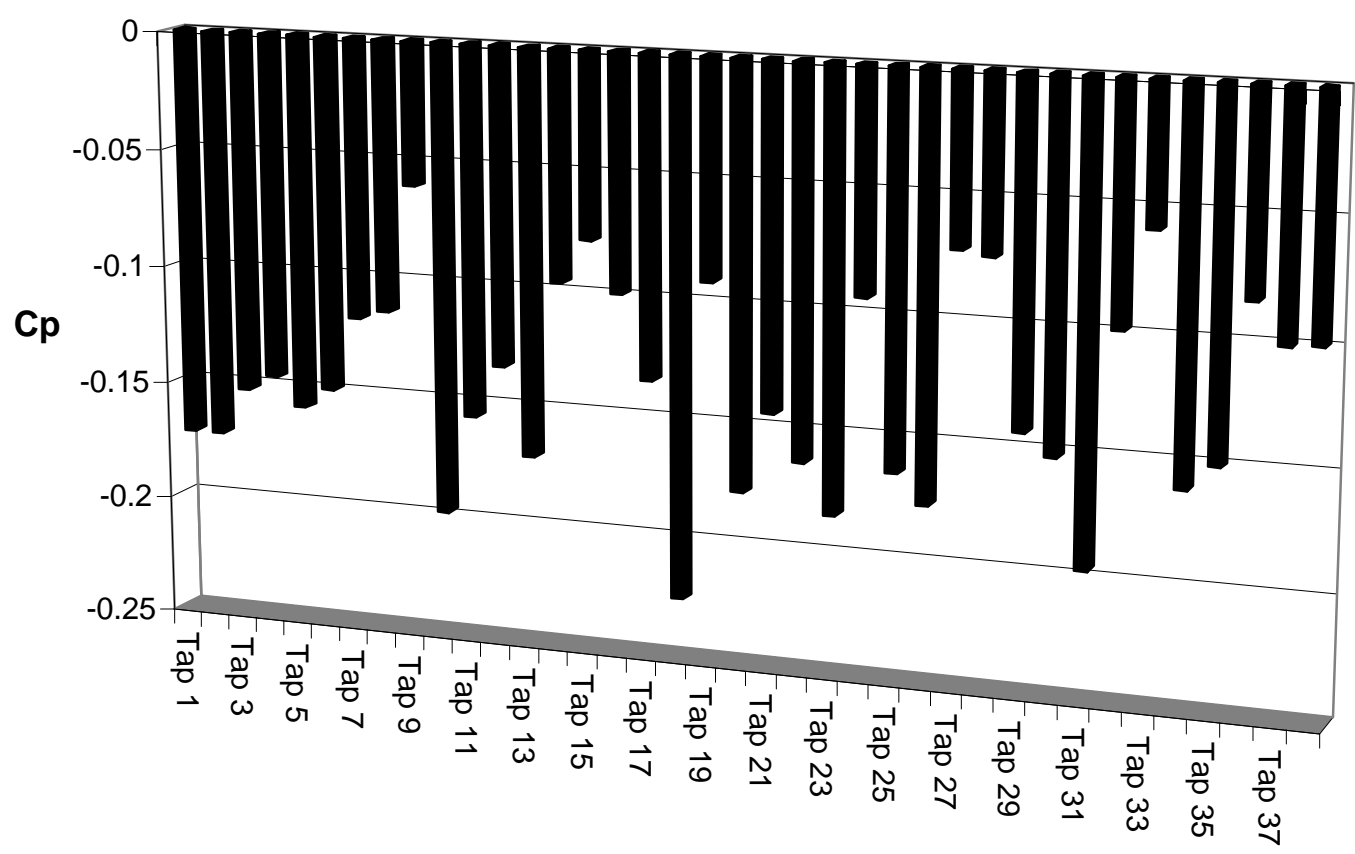

Figure 5.11: Phase I Average Pressure Coefficients for $1 / 8$-inch VG Modified Configuration 1 Test.

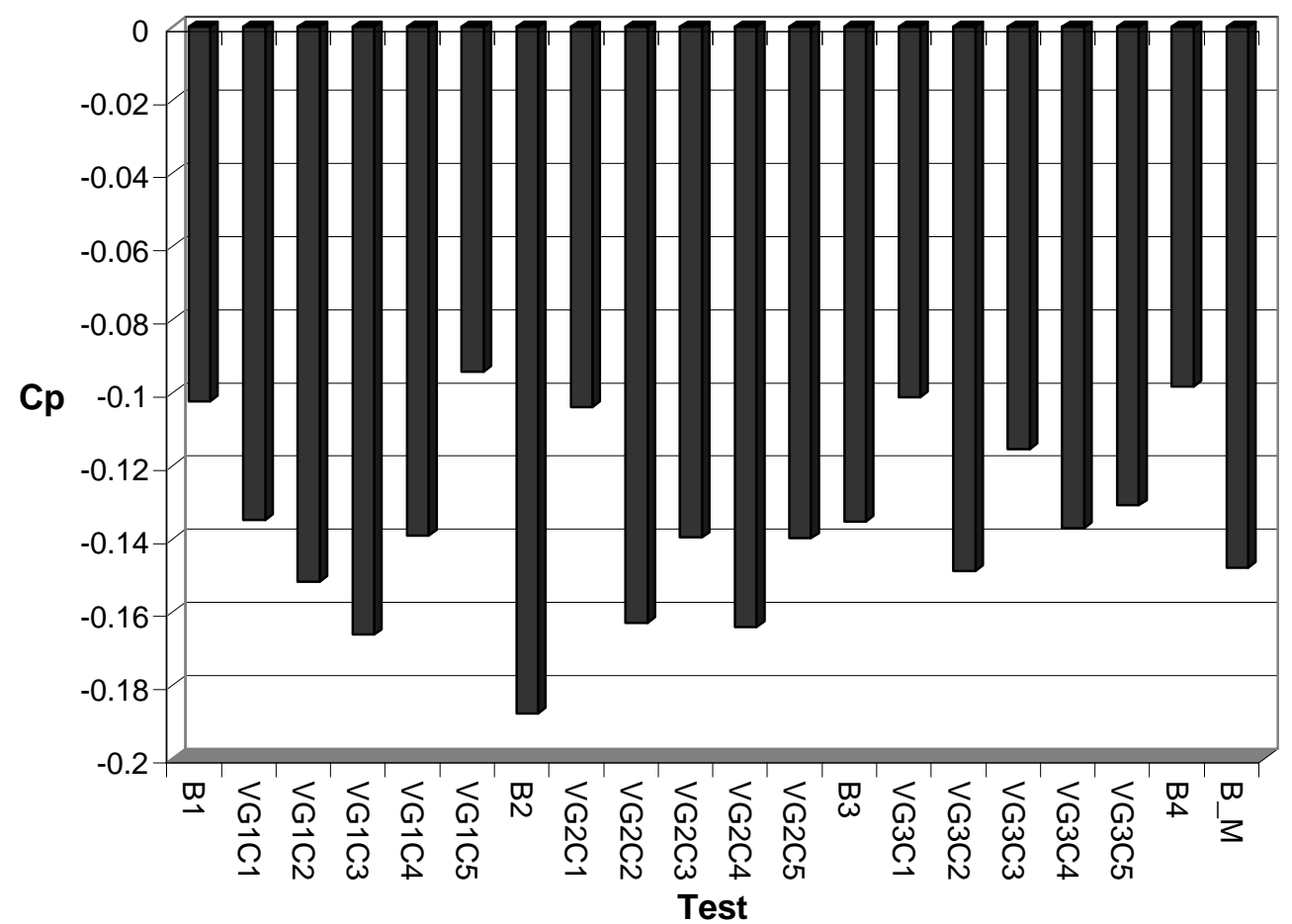

Figure 5.12: Comparison of Phase I Average Pressure Coefficients for Pressure Tap \#1. 


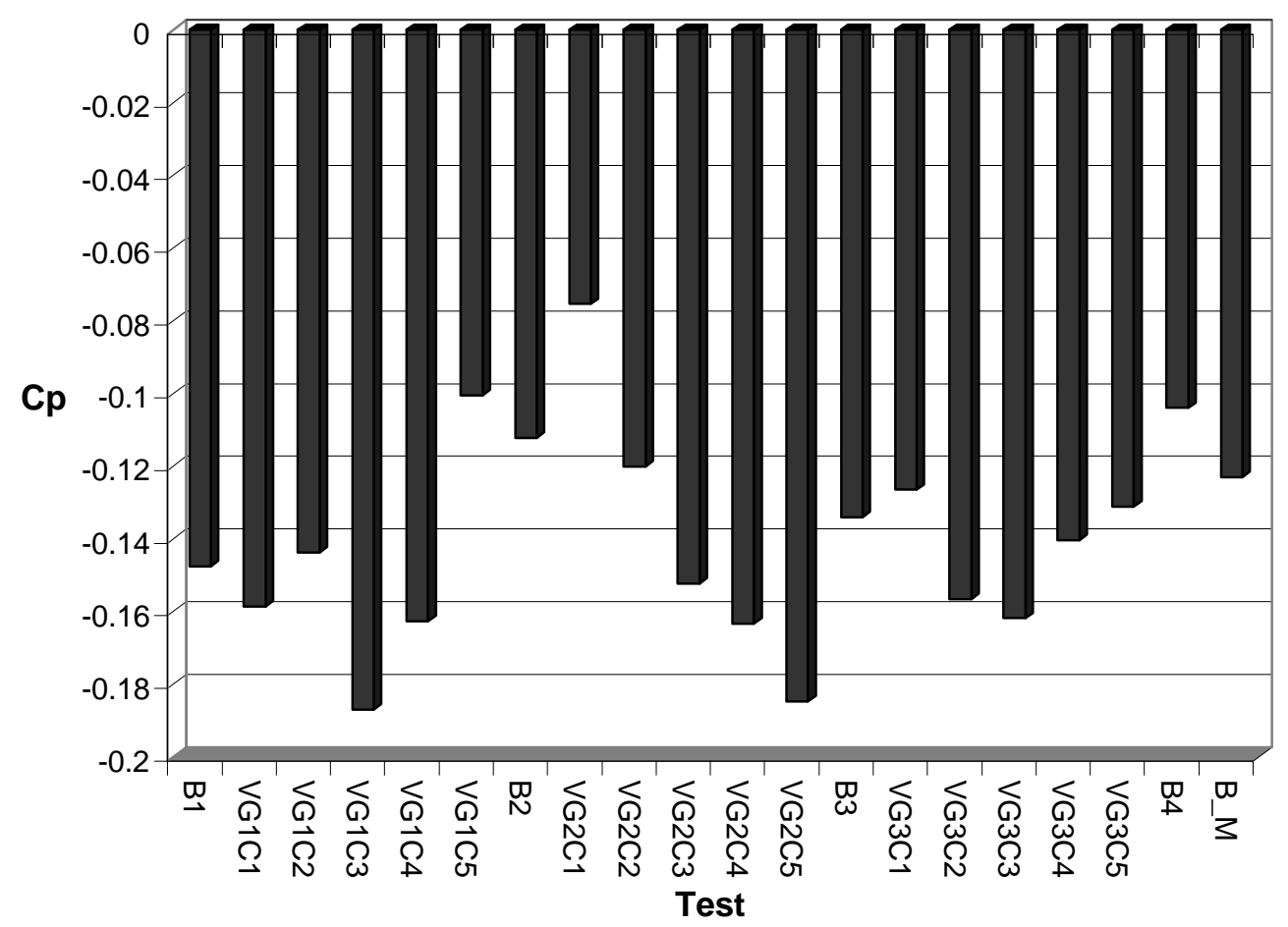

Figure 5.13: Comparison of Phase I Average Pressure Coefficients for Pressure Tap \#23.

\subsection{Phase II Results}

Testing conducted in the Langley Full Scale Wind Tunnel with the cooperation of Old Dominion University and Dr. Drew Landman for Phase II had marginal results. The limited results in this portion of testing are primarily due to the limited time and high cost of testing in the LFST. In the time allotted blockage effects were the first consideration and more testing time in this facility would be needed to determine a drag reducing VG configuration. As seen in Figure 5.14 most configurations showed a drag increase, and those with a drag decrease were within the instrumentation error $\left( \pm 2 \%\right.$ of $\left.\mathrm{C}_{\mathrm{P}}\right)$. The actual values of the drag coefficient and each configuration change from the average baseline drag coefficient are listed in Table 5.2 for the $55 \mathrm{ft} / \mathrm{s}$ case. Table 5.3 covers the $\mathrm{C}_{\mathrm{D}}$ and 
$\Delta \mathrm{C}_{\mathrm{D}}$ values for the $70 \mathrm{ft} / \mathrm{s}$, and the $120 \mathrm{ft} / \mathrm{s}$ case are listed in Table 5.4. Again, there are only marginal changes in the drag coefficient for Phase II testing at all three airspeeds.

Table 5.2: Drag Coefficients of the $55 \mathrm{ft} / \mathrm{s}$ tests Conducted in the LFST, Phase II.

\begin{tabular}{|c|c|c|}
\hline Test & $\mathrm{C}_{\mathrm{d}}$ & $\Delta \mathrm{C}_{\mathrm{d}}$ \\
\hline Baseline & 0.5051 & ------- \\
\hline Baseline2 & 0.5072 & ------ \\
\hline VG1C4 & 0.5062 & 0.0004 \\
\hline VG1C5 & 0.5176 & 0.0118 \\
\hline VG2C4 & 0.5087 & 0.0029 \\
\hline VG2C5 & 0.5086 & 0.0028 \\
\hline VG3C4 & 0.5042 & -0.0016 \\
\hline VG3C5 & 0.5074 & 0.0016 \\
\hline
\end{tabular}

Table 5.3: Drag Coefficients of the $70 \mathrm{ft} / \mathrm{s}$ tests Conducted in the LFST, Phase II.

\begin{tabular}{|c|c|c|}
\hline Test & $\mathrm{C}_{\mathrm{d}}$ & $\Delta \mathrm{C}_{\mathrm{d}}$ \\
\hline Baseline & 0.5067 & ------- \\
\hline Baseline2 & 0.5048 & $-\cdot----$ \\
\hline VG1C4 & 0.5056 & 0.0001 \\
\hline VG1C5 & 0.5132 & 0.0077 \\
\hline VG2C4 & 0.5059 & 0.0004 \\
\hline VG2C5 & 0.5070 & 0.0015 \\
\hline VG3C4 & 0.5047 & -0.0008 \\
\hline VG3C5 & 0.5063 & 0.0008 \\
\hline
\end{tabular}

Table 5.4: Drag Coefficients of the $120 \mathrm{ft} / \mathrm{s}$ tests Conducted in the LFST, Phase II.

\begin{tabular}{|c|c|c|}
\hline Test & $\mathrm{C}_{\mathrm{d}}$ & $\Delta \mathrm{C}_{\mathrm{d}}$ \\
\hline Baseline & 0.5054 & $-\cdot-----$ \\
\hline Baseline2 & 0.5054 & ------- \\
\hline VG1C4 & 0.5069 & 0.0015 \\
\hline VG1C5 & 0.5119 & 0.0065 \\
\hline VG2C4 & 0.5047 & -0.0007 \\
\hline VG2C5 & 0.5095 & 0.0041 \\
\hline VG3C4 & 0.5080 & 0.0026 \\
\hline VG3C5 & 0.5079 & 0.0025 \\
\hline
\end{tabular}

The dependence of the drag coefficient on the airspeed for the baseline tests is shown in Figure 5.15. The differences in the drag coefficient measured for these tests 
falls within the $2 \%$ error in the measurements, which is shown by the error bars on the plot. Figure 5.16 shows the velocity dependence of the 1/2-inch VG for the two configurations that were tested in the LFST. Again, the difference in the drag coefficient is within the instrumentation error for both the speed dependence and the configuration change. A similar trend is present for the 1/4-inch, Figure 5.17, and the 1/8-inch vortex generators, Figure 5.18.

Unfortunately due to instrumentation problems with the pressure transducer in the Langley Full Scale Tunnel, pressure data was not taken for the first three configurations of the test matrix. These tests were the first baseline test and the $1 / 2$-inch vortex generator tests. By the fourth test, with the 1/4-inch vortex generator, the transducer was working properly, and thus pressure data was collected for the remaining tests. Pressure Tap Number 8 was damaged during transport and was not re-attached for Phase II testing.

Figure 5.19 shows the pressure distribution for run 4.02, Configuration 4 for the 1/4-inch VG at an airspeed of $70 \mathrm{ft} / \mathrm{s}$. Again, there are certain taps that have a higher negative pressure, not all of which are in the same lengthwise location. Configuration 5 with the 1/4-inch device shows a similar trend with the airspeed as seen in Figures 5.20 (Run 5.02) for the $70 \mathrm{ft} / \mathrm{s}$ test. Extremely small changes in the pressure coefficient from Configuration 4 to Configuration 5, which leads to several possible explanations, covered in the Conclusions section.

The 1/8-inch VG for Configuration 4 pressure distributions are shown in Figure 5.21 is for the $120 \mathrm{ft} / \mathrm{s}$ test (Run 6.03). The CP's for run 7.03 (1/8-inch VG, Configuration 5) are shown in Figure 5.22. Again, there were minimal changes in the pressure coefficients between the two configurations and minimal effect on the drag 
coefficient. The pressure coefficients for the second baseline test (Runs 8.01) are shown in Figures 5.23. Changes are noticeable only on several pressure taps. Comparing this run to the previous configurations test, there is little difference from the baseline pressure coefficients, thus it is reasonable that the drag coefficients are so close. More explanation into the cause of the discrepancy between Phase I and Phase II results are discussed in the Conclusions Chapter of this thesis.

In attempts to find some configuration that would work in reducing the drag two new configurations were tested. At this time the placement of the vortex generators is still a trial and error process. Run 9.01 was run with the 1/2-inch vortex generators located immediately downstream of the seat to try to reattach the airflow on the rear fairing. Figure 5.24 shows the pressure distribution for this test, but due to the tap locations the VG's had no influence on the pressure coefficients compared to the baseline configuration. The pressure distribution for Run 10.01 is shown in Figure 5.25. The VG placement for this test was the same as for Run 9.01 except for 6 pairs of VG's added on the lower leading edge of the lower fairing. Again there was little variation from the baseline pressure coefficients. This small change in pressure helps explain the limited change in drag coefficient. Table 5.5 lists the drag coefficient and the change for the average baseline drag coefficient for Runs 9.01 and 10.01 .

Table 5.5: Drag Coefficients for the New Configurations Tested in the LFST.

\begin{tabular}{|c|c|c|}
\hline Test & Cd & $\Delta \mathrm{Cd}$ \\
\hline Run 9.01 & 0.5059 & 0.0005 \\
\hline Run 10.01 & 0.5060 & 0.0006 \\
\hline
\end{tabular}

To better visualize the effects of VG placement and airspeed on the pressure distribution Figures 5.26 was created for Tap Location Number 5. The pressure is more 
positive and there is a minor dependence on the vortex generator location and a slightly larger dependence on the airspeed, as seen in the figure. Pressure Tap Number 26, along the trailing edge of the lower fairing, experienced almost no change from test to test but shows the trend with airspeed where increasing speed increased the pressure coefficient in the negative direction, as seen in Figure 5.27.

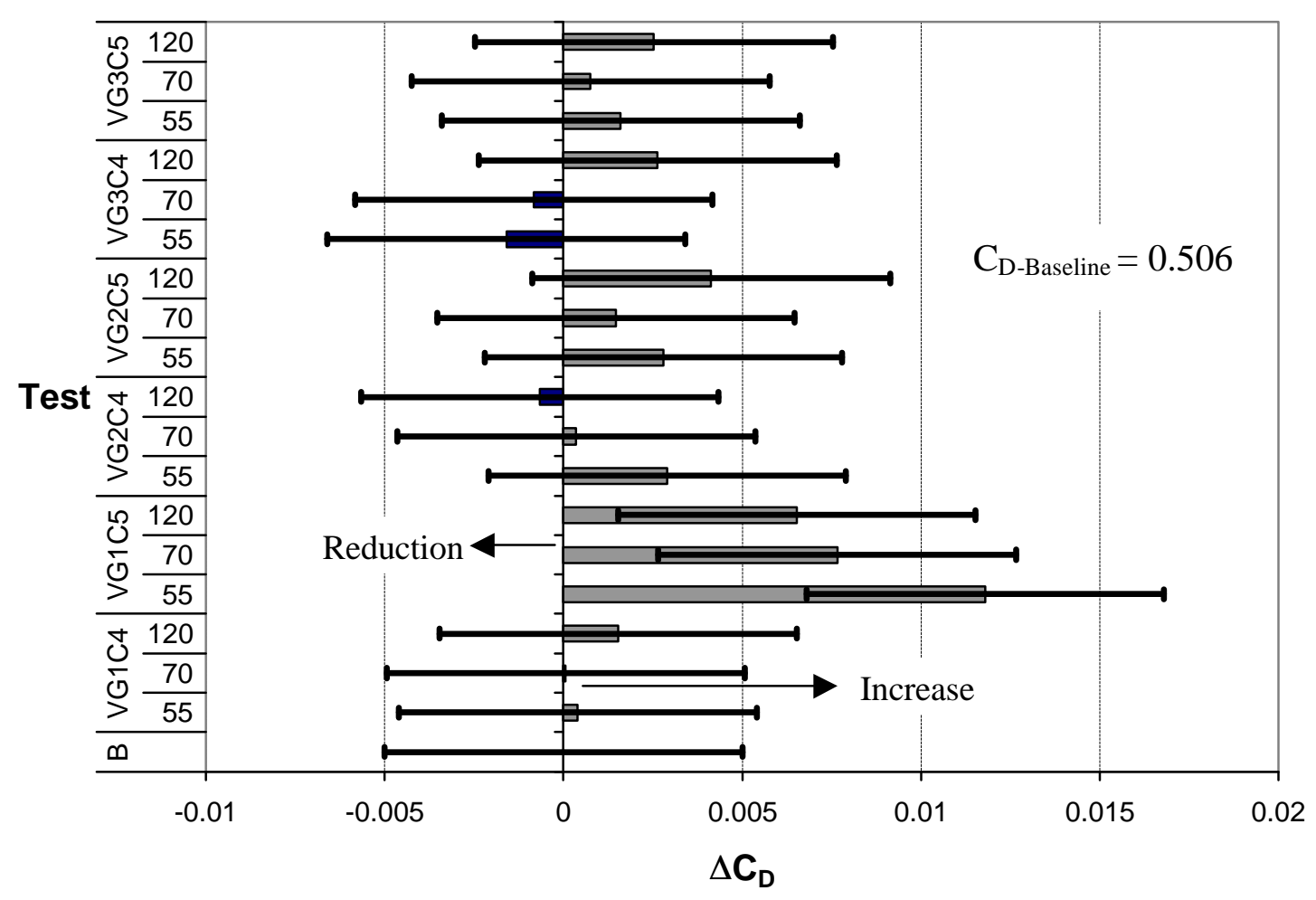

Figure 5.14: Comparison of Relative Drag Coefficients for Phase II of Testing, Conducted in the Langley Full Scale Wind Tunnel. 


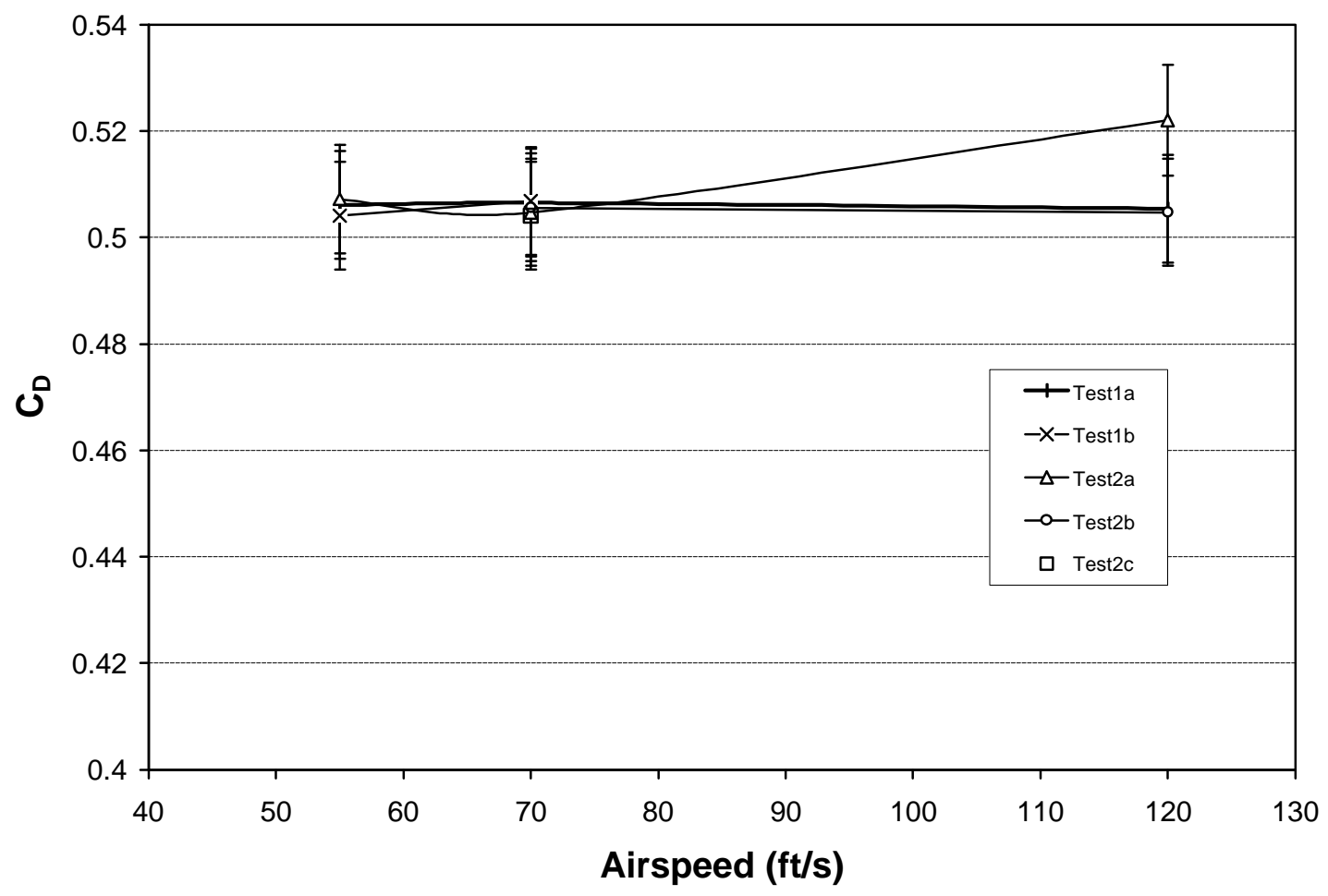

Figure 5.15: Variation in Drag Coefficient with Free stream Velocity for the Phase II Baseline Configuration.

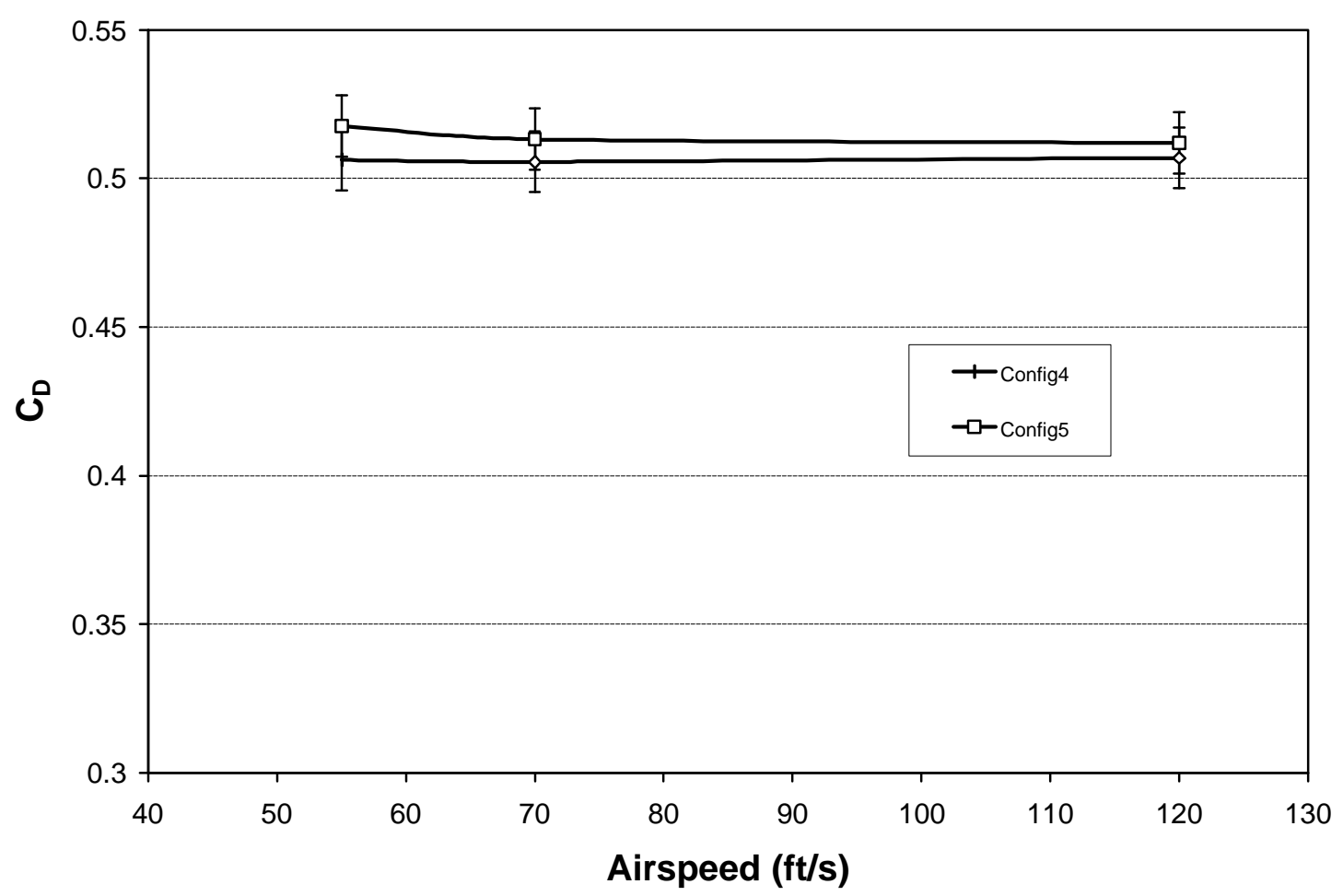

Figure 5.16: Variation in Drag Coefficient with Free stream Velocity for the Phase II 1/2-inch Vortex Generator Configurations 4 and 5. 


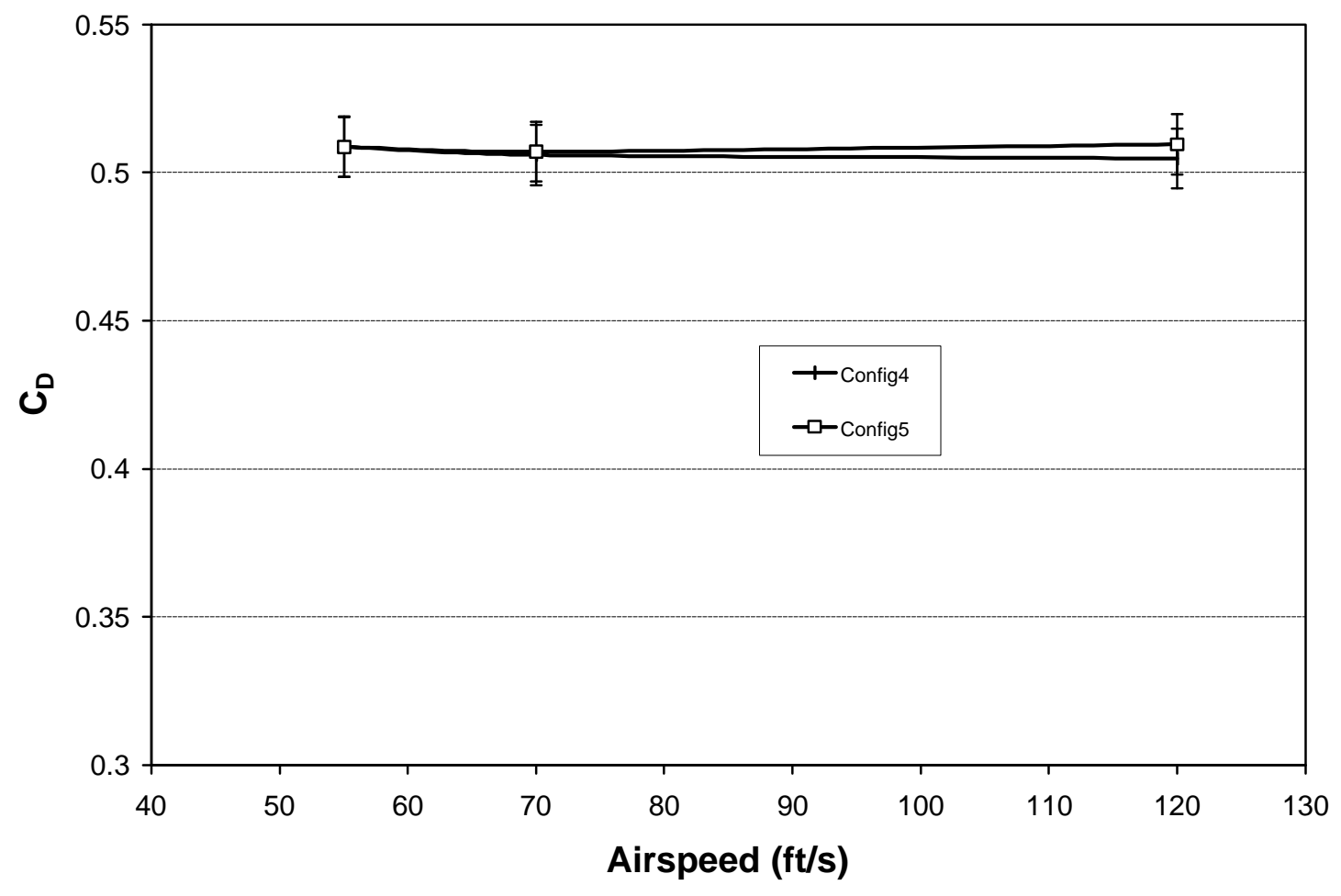

Figure 5.17: Variation in Drag Coefficient with Free stream Velocity for the Phase II 1/4-inch Vortex Generator Configurations 4 and 5.

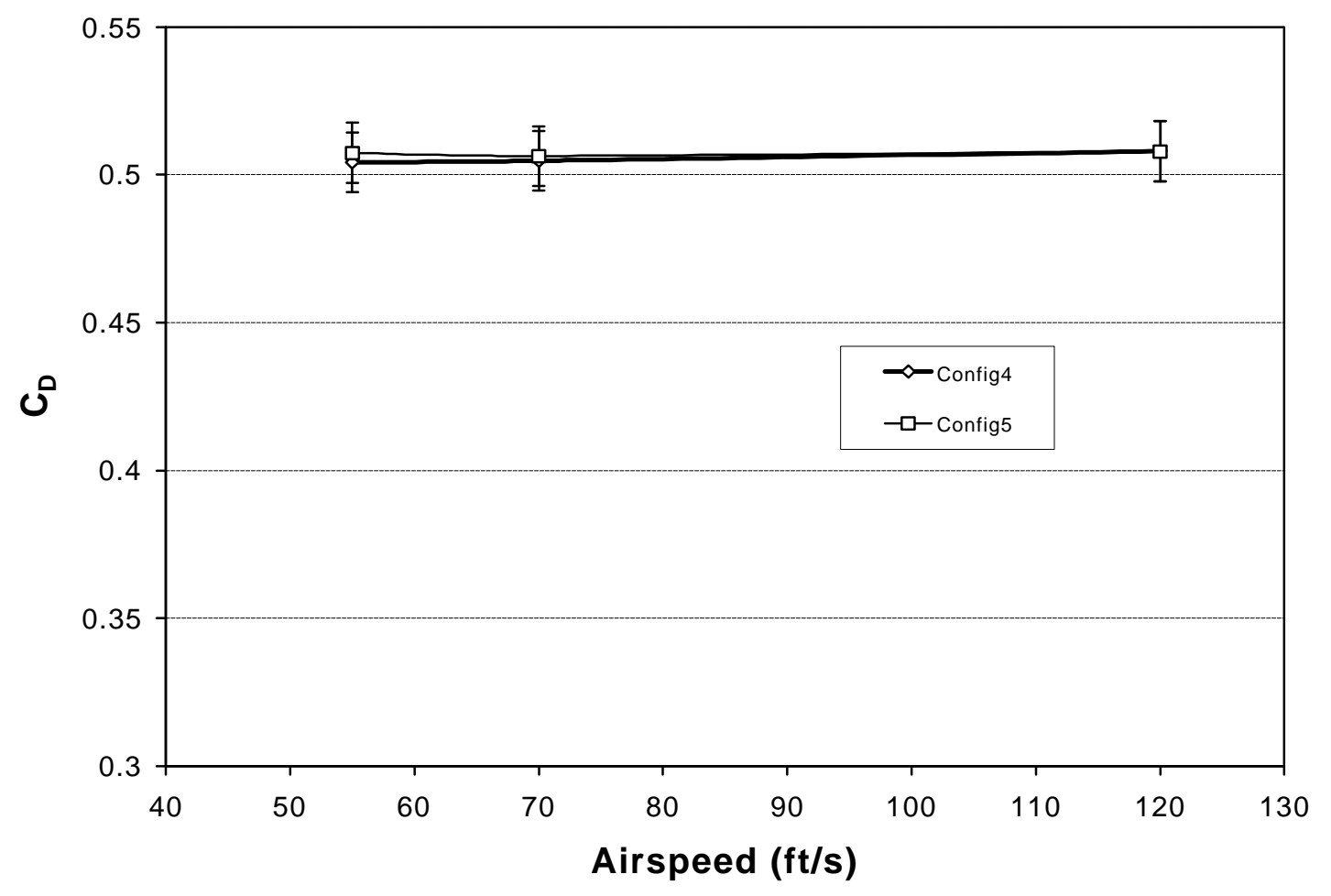

Figure 5.18: Variation in Drag Coefficient with Free stream Velocity for the Phase II 1/8-inch Vortex Generator Configurations 4 and 5. 


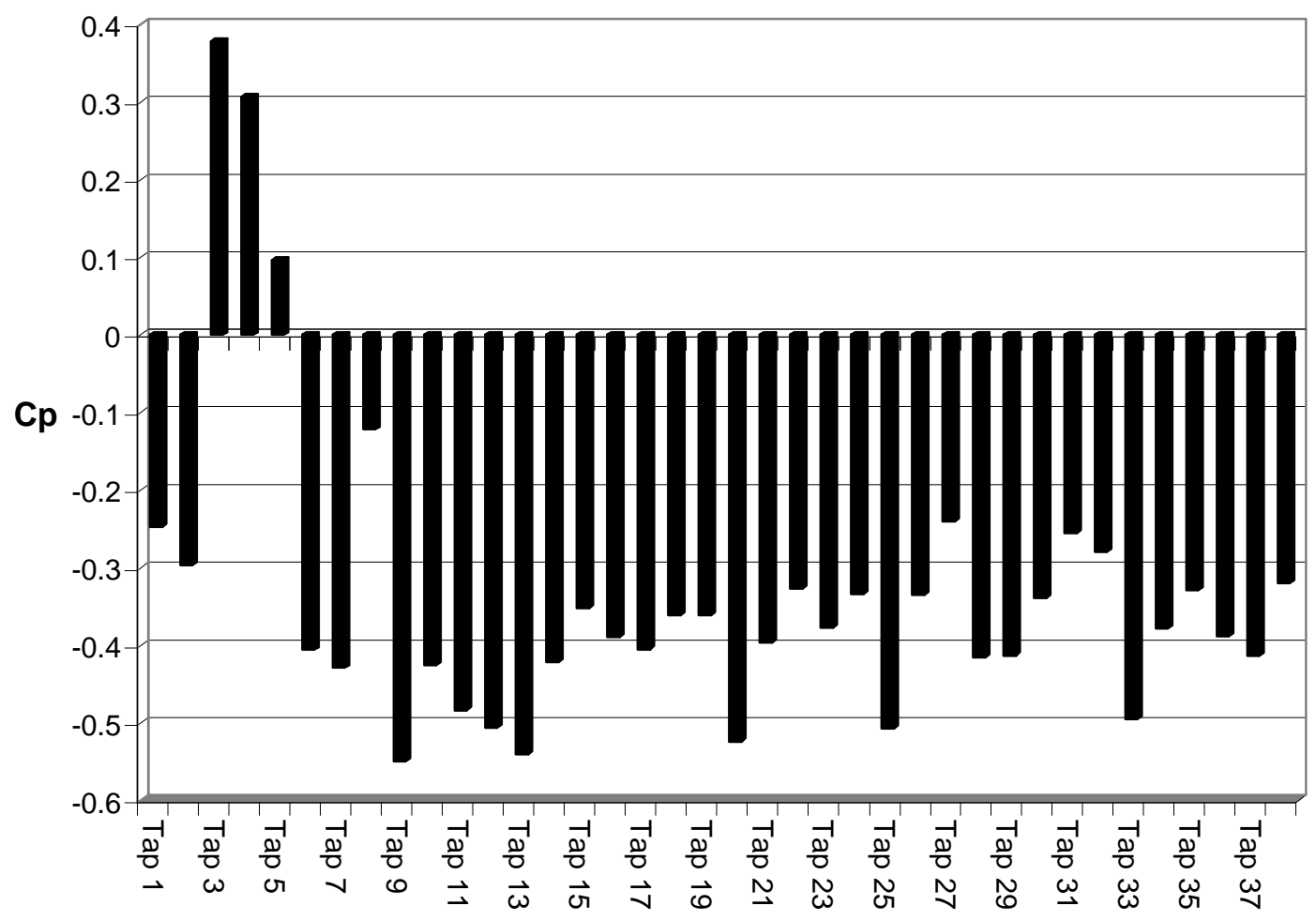

Figure 5.19: Average Pressure Coefficients for Phase II Run 4.02.

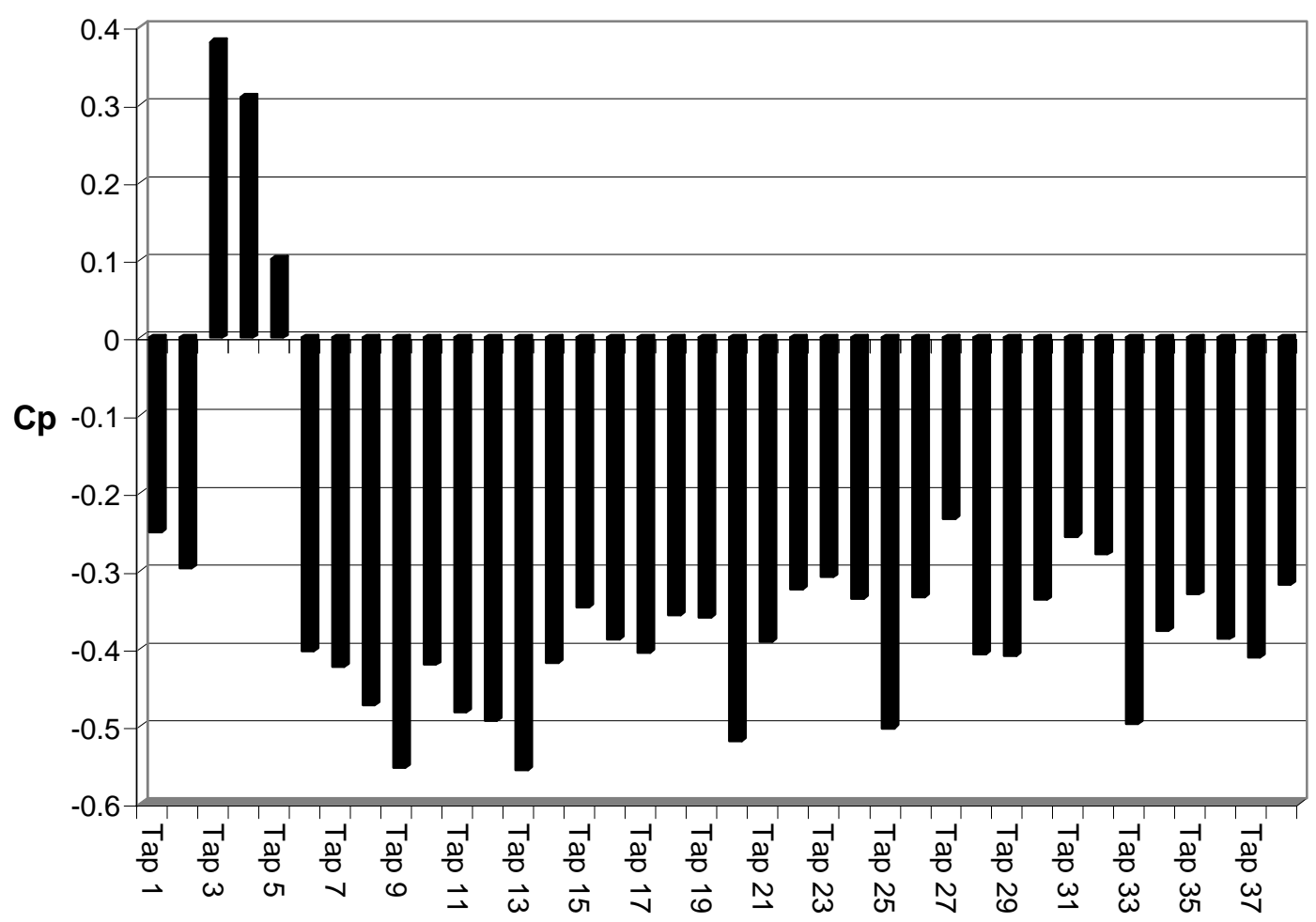

Figure 5.20: Average Pressure Coefficients for Phase II Run 5.02. 


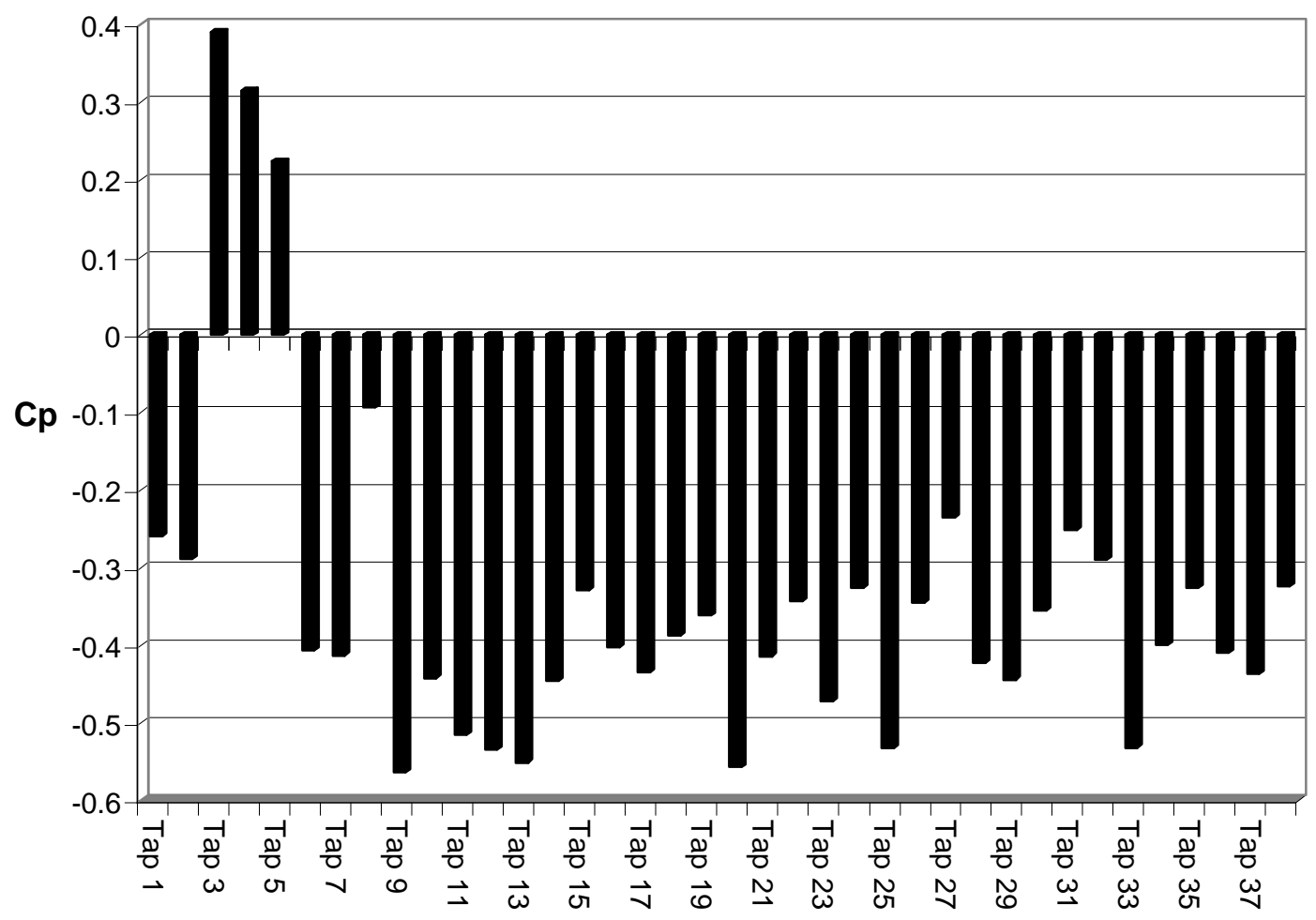

Figure 5.21: Average Pressure Coefficients for Phase II Run 6.03.

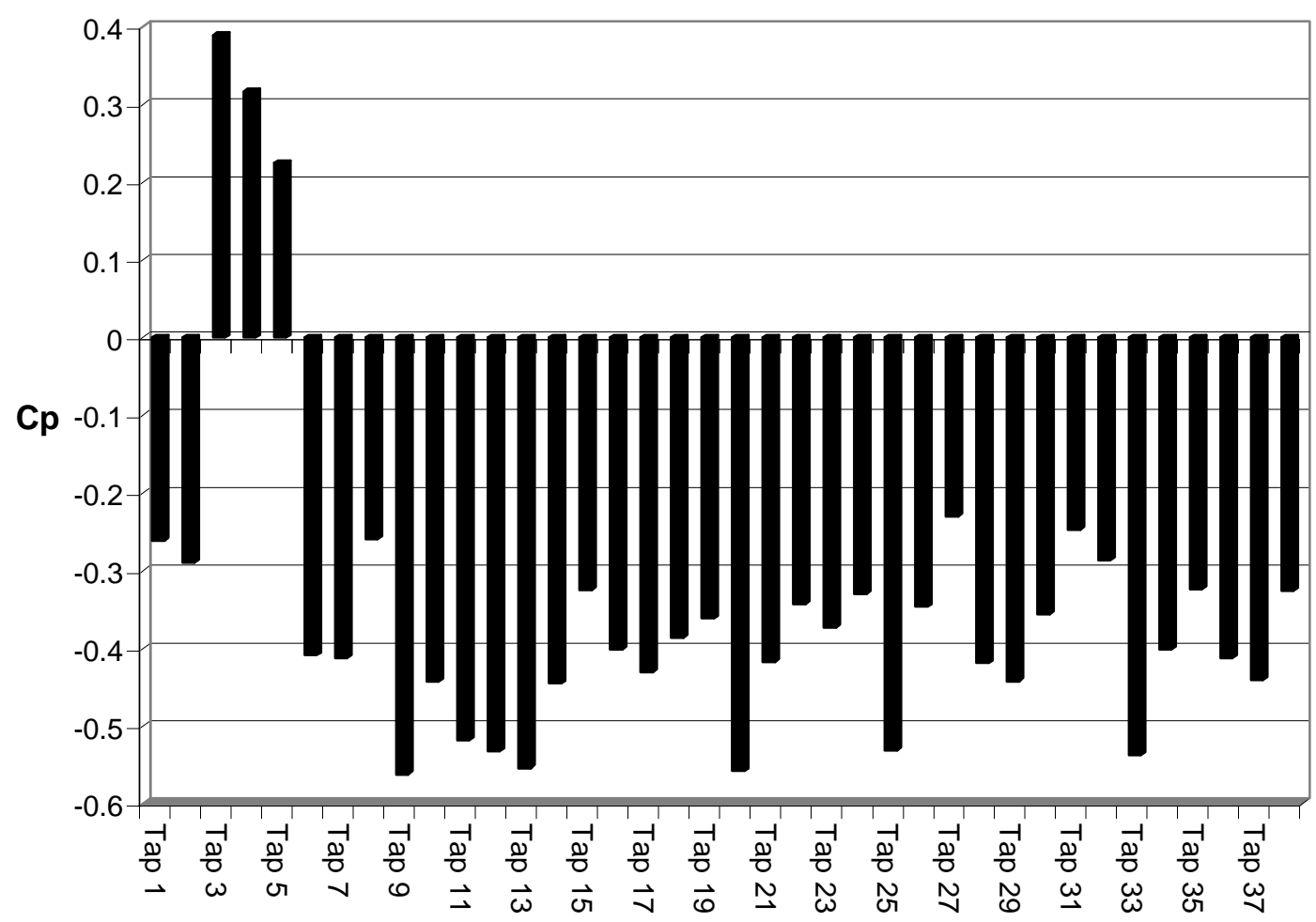

Figure 5.22: Average Pressure Coefficients for Phase II Run 7.03. 


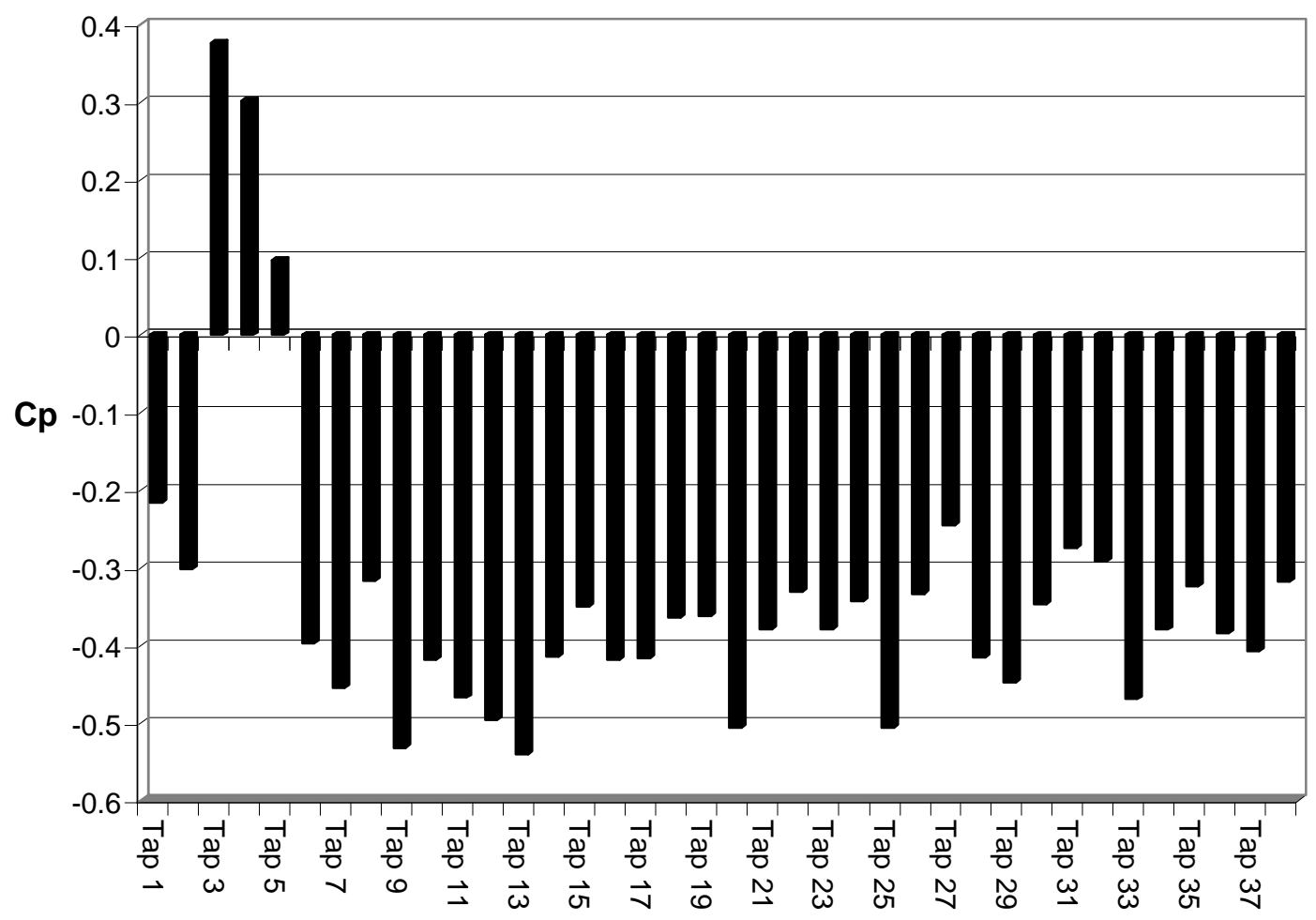

Figure 5.23: Average Pressure Coefficients for Phase II Run 8.01.

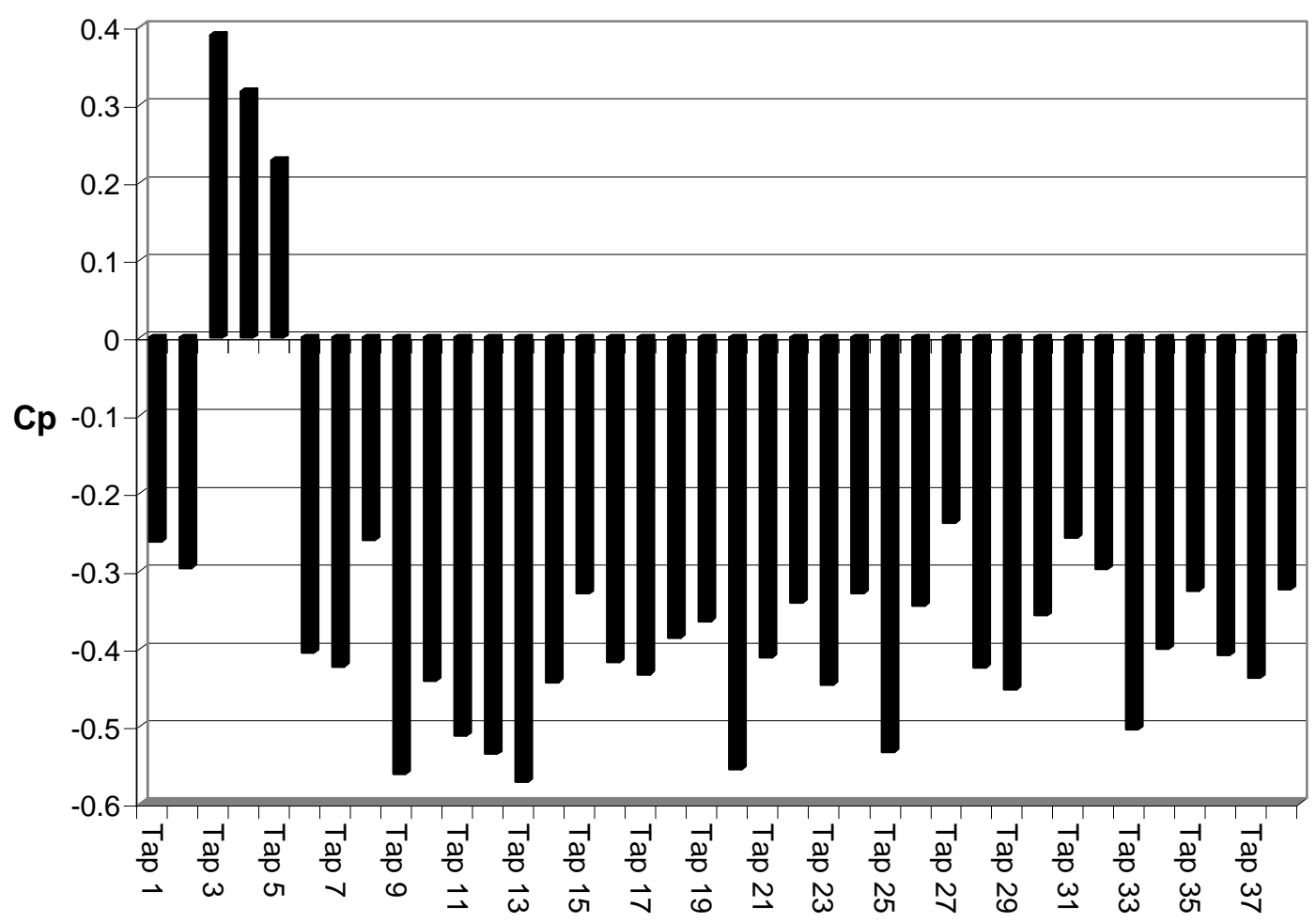

Figure 5.24: Average Pressure Coefficients for Phase II Run 9.01. 


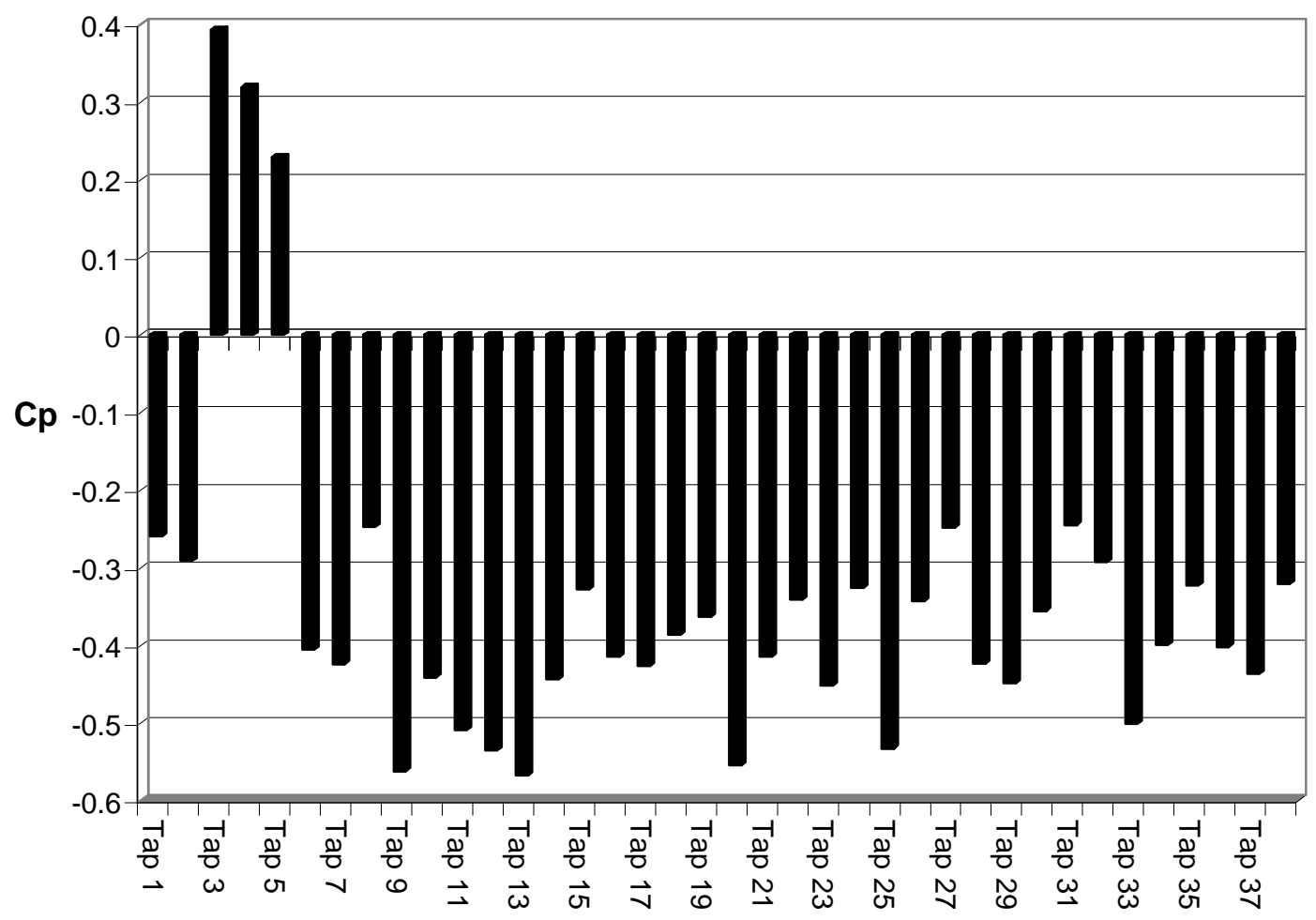

Figure 5.25: Average Pressure Coefficients for Phase II Run 10.01.

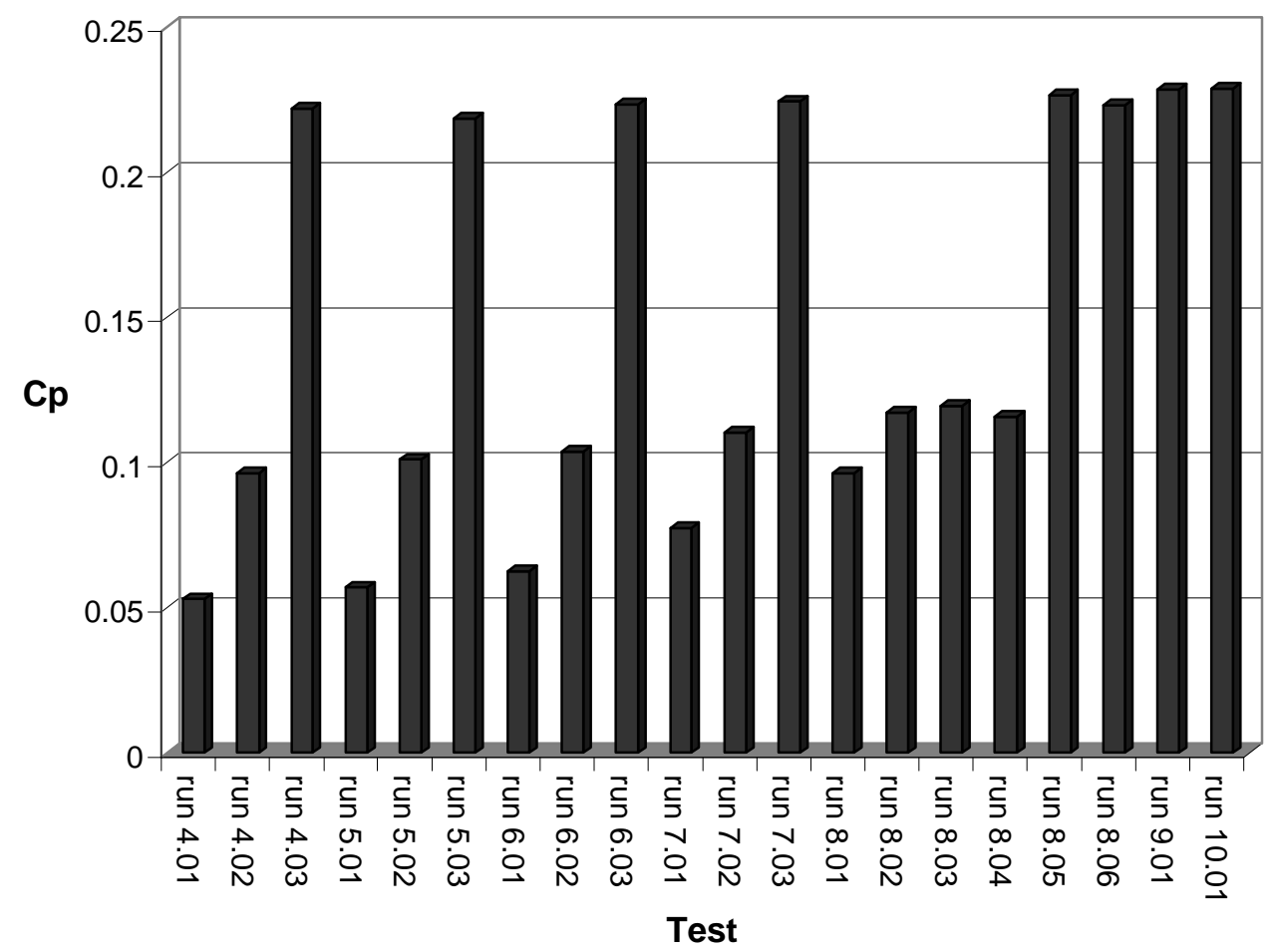

Figure 5.26: Comparison of Phase II Average Pressure Coefficients for Pressure Tap \#5. 


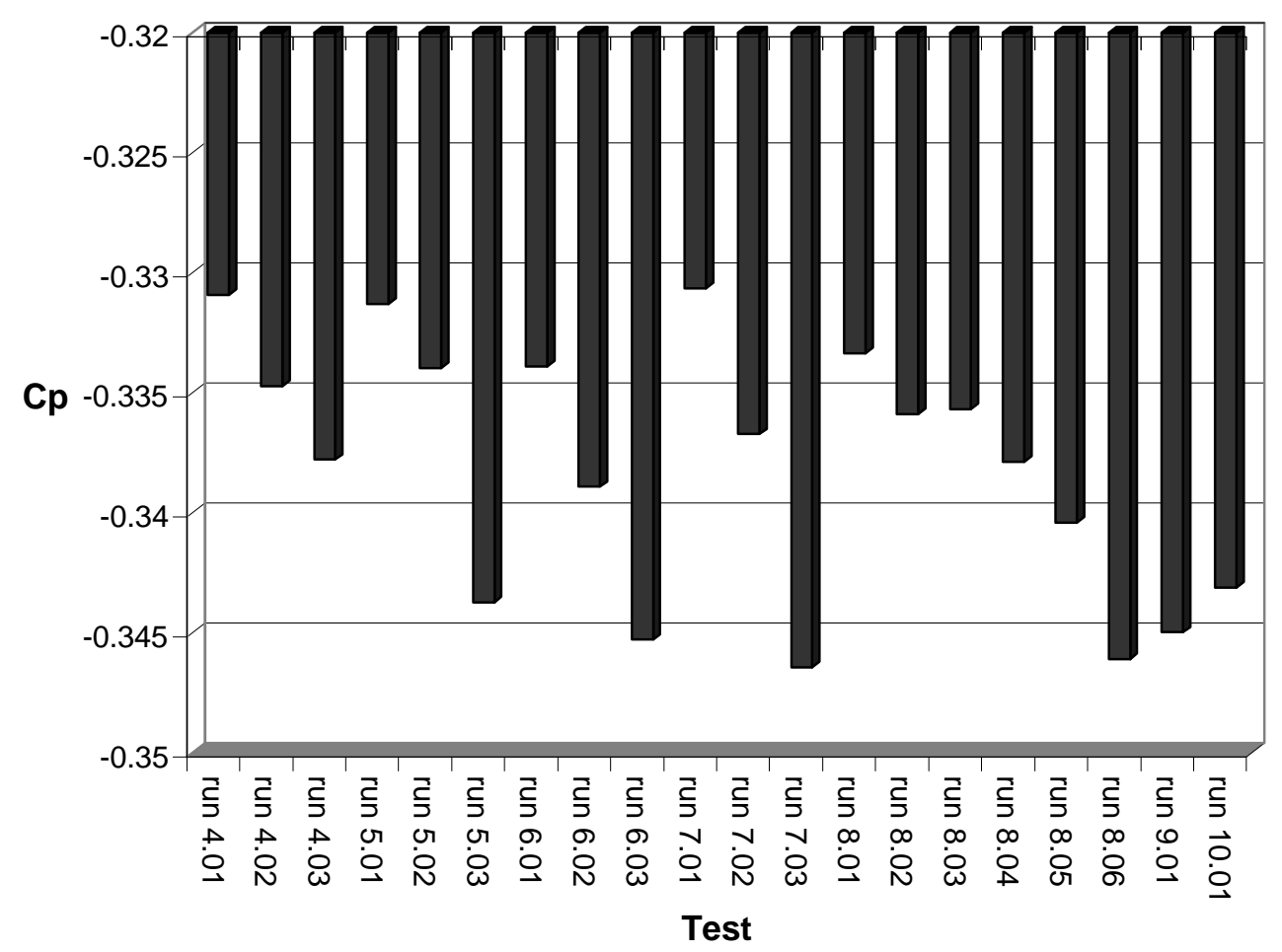

Figure 5.27: Comparison of Phase II Average Pressure Coefficients for Pressure Tap \#26.

\subsection{Phase III Results}

Tests for Phase III were conducted in the WVU Closed Loop Wind Tunnel. Figure 5.28 shows the average measured change in drag coefficient for the 12 tests conducted for each configuration during this phase of testing. From this figure it is seen that the 1/2-inch vortex generator increases the drag coefficient over the baseline configuration. Similarly, the $1 / 4$-inch VG increases the drag coefficient but Configuration 5 has only half the increase of Configuration 4. Unlike the Phase I results the drag coefficient for two of the $1 / 8$-inch VG configurations experienced a small drag increase; possible causes of this are discussed in the Conclusions. 
The parametric study on the upper surface (OVG3P) with 4 pairs of vortex generators started at the trailing edge with a small increase in drag and worked toward the leading edge. Parametric Position Number 3, which was approximately 10 inches from the leading edge recorded the greatest reduction in drag coefficient of these four tests. A similar parametric study was conducted on the lower fairing (OLFP) with 3 pairs of VG's was tested at three locations starting at the leading edge. These tests all show a reduction in relative drag coefficient, from moving the VG's upstream it was noticed that the position near the center of the lower fairing showed the largest drag reduction. It was expected that the VG size should be smaller than the boundary layer thickness, which is supported by the data where near the leading edge of the fairing there is a smaller reduction in drag. The decrease in drag reduction further downstream can be explained by the increased thickness in the boundary layer making the VG less effective by reducing the ratio of VG height to boundary layer thickness. Therefore, it is best to place the VG where its height is approximately the boundary layer height, or slightly smaller than the boundary layer.

Figure 5.29 shows the dependence of the drag coefficient on the position of the vortex generator on the upper fairing. These tests were conducted with no vortex generators of the lower fairing. From this parametric study it was determined that, depending on where it is placed, the $1 / 8$-inch vortex generator can either increase on decrease the drag on the model. The lower fairing was also parametrically studied as seen in Figure 5.30, where the center position resulted in the lowest drag reduction.

In an effort to investigate blockage effects, sheets of 1-inch thick foam were added to the walls to reduce the test section cross-sectional area. The standard deviations 
in the measured drag coefficients are listed in Table 5.6, along with the average change in drag for the tests conducted. Figure 5.31 shows the measured difference in drag coefficient for the two test section areas. Table 5.7 lists the change in drag coefficient and the test section areas for the two sets of tests. From this testing is was determined that test section blockage as a considerable effect on the effectiveness of the vortex generators.

Table 5.6: Measured Change in Drag Coefficient and the Corresponding Standard Deviation for the Given Number of Tests Conducted During Phase III.

\begin{tabular}{|c|c|c|c|}
\hline Test & Num. Of Tests & $\Delta \mathrm{C}_{D}$ & StDev. \\
\hline OVG1C1 & 4 & 0.2174 & 0.5619 \\
\hline OVG1C2 & 4 & 0.1868 & 5.4781 \\
\hline OVG2C3 & 4 & 0.1538 & 0.7966 \\
\hline OVG2C4 & 12 & 0.0594 & 9.6798 \\
\hline OVG3C4 & 12 & 0.0679 & 3.3087 \\
\hline OVG3C5 & 12 & 0.0441 & 5.6387 \\
\hline OVG3M1 & 12 & 0.04 & 7.0014 \\
\hline OVG3P1 & 12 & 0.0208 & 3.6601 \\
\hline OVG3P2 & 12 & 0.014 & 6.4425 \\
\hline OVG3P3 & 12 & -0.0416 & 10.550 \\
\hline OVG3P4 & 12 & -0.0279 & 9.0380 \\
\hline OLFP1 & 4 & -0.027 & 0.5749 \\
\hline OLFP2 & 4 & -0.0475 & 0.7691 \\
\hline OLFP3 & 4 & -0.0414 & 0.3825 \\
\hline TSARVG1C2 & 4 & -0.0053 & 0.0158 \\
\hline TSARVG3P3 & 4 & 0.02675 & 0.0034 \\
\hline
\end{tabular}

Table 5.7: Effect of Blockage on Drag Coefficient and the Associated Test Section Area.

\begin{tabular}{|c|c|c|}
\hline Test & T.S.Area $\left(\mathrm{in}^{2}\right)$ & $\Delta \mathrm{C}_{\mathrm{D}}$ \\
\hline \multirow{2}{*}{ VG1C2 } & 3689.969 & 0.187 \\
\cline { 2 - 3 } & 3542.125 & -0.005 \\
\hline \multirow{2}{*}{ VG3P3 } & 3689.969 & -0.042 \\
\cline { 2 - 3 } & 3542.125 & 0.027 \\
\hline
\end{tabular}




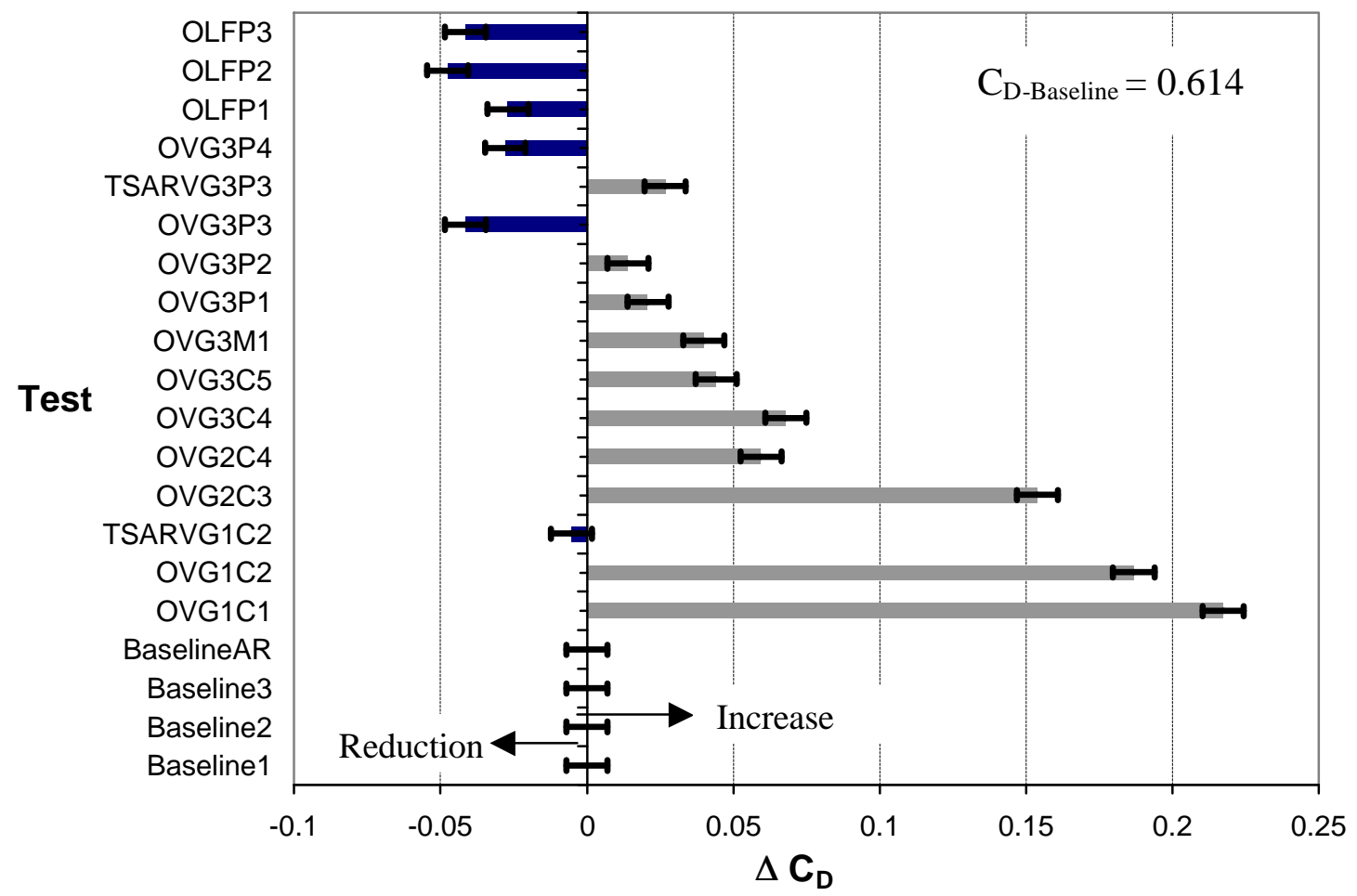

Figure 5.28: Comparison of Relative Drag Coefficients for Phase III of Testing Conducted in the West Virginia University Closed Loop Wind Tunnel.

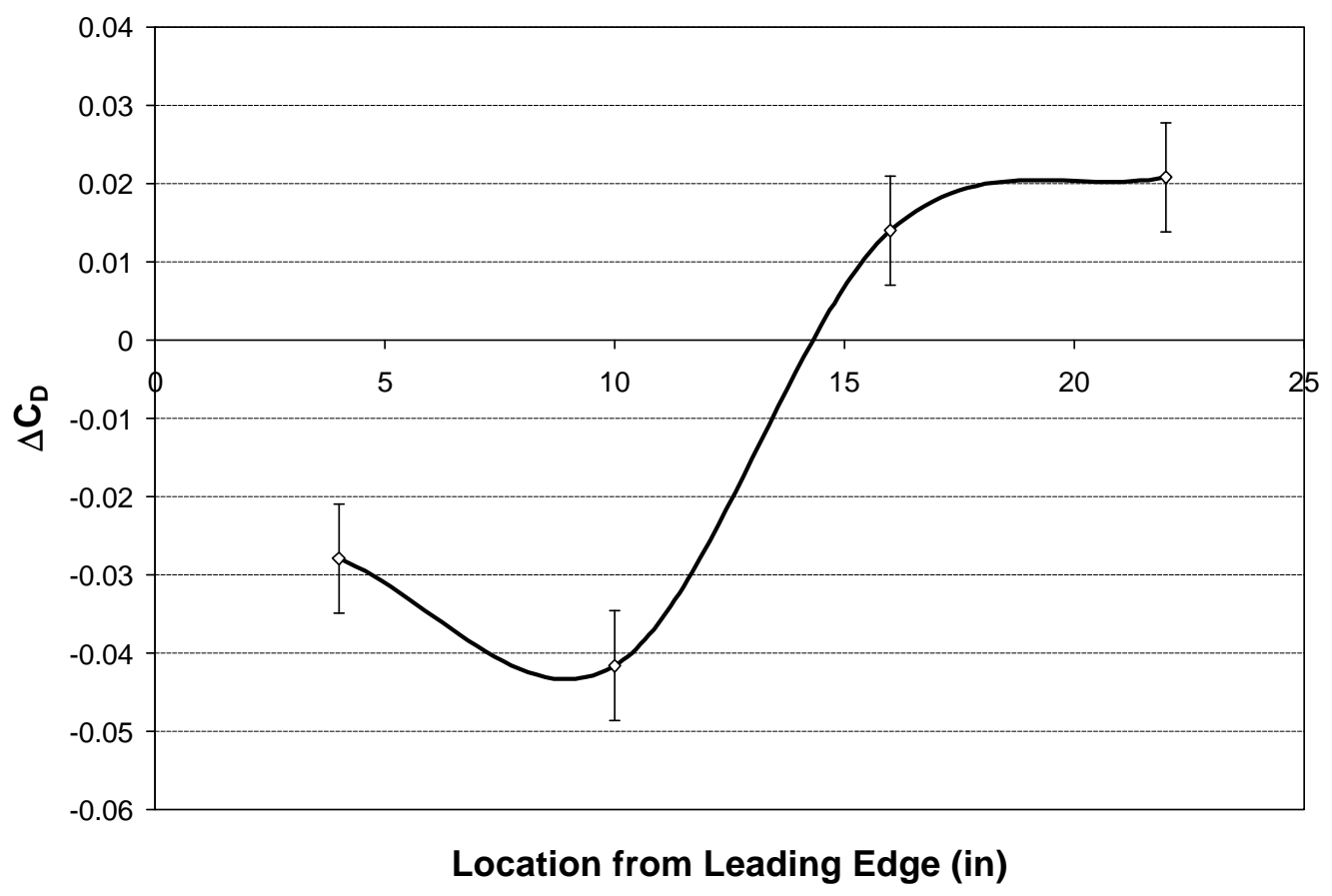

Figure 5.29: Upper Fairing Parametric Plot of Drag Coefficient as Dependent of Vortex Generator Location from the Leading Edge. 


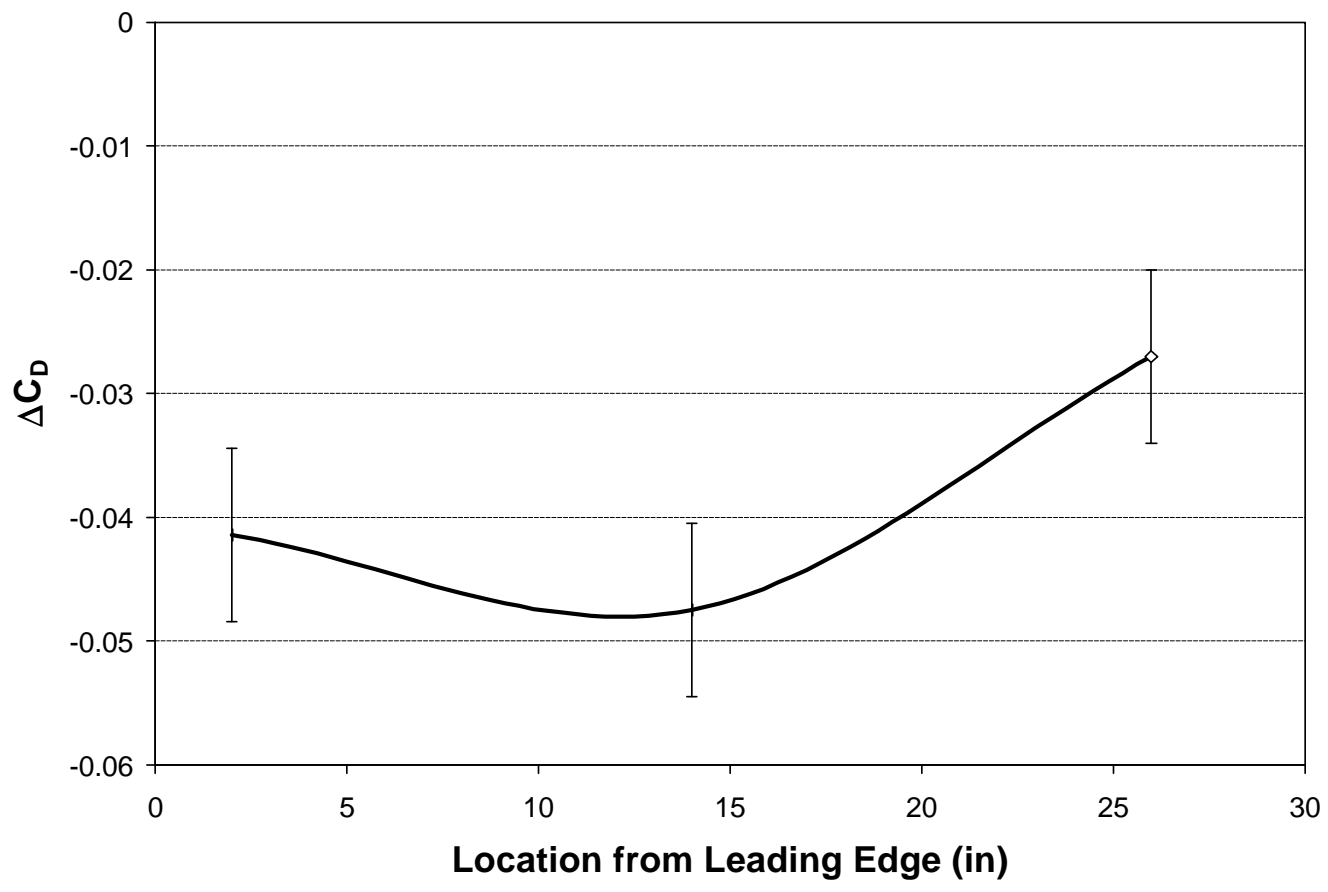

Figure 5.30: Lower Fairing Parametric Plot of Drag Coefficient as Dependent of Vortex Generator Location from the Leading Edge.

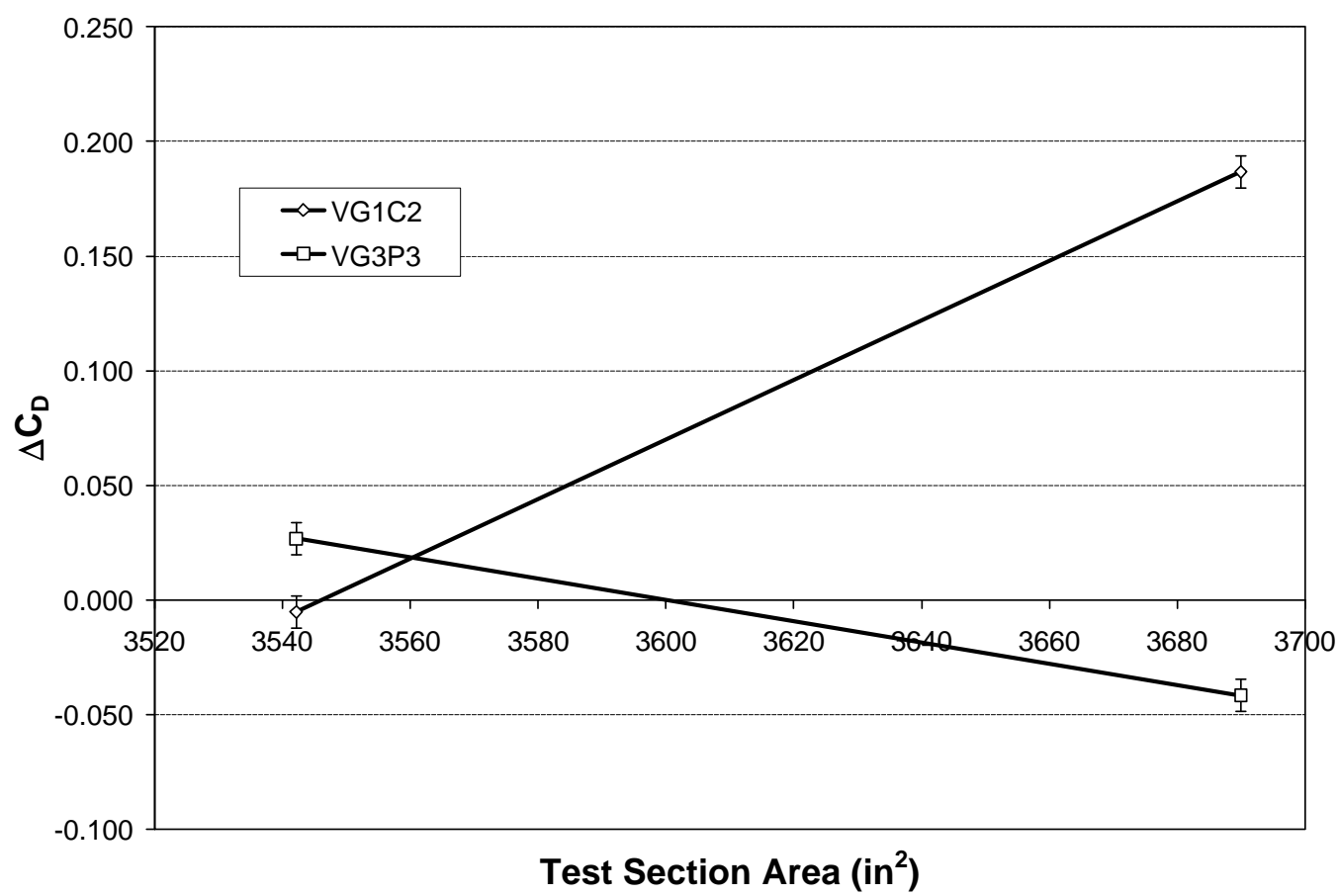

Figure 5.31: Effects of Test Section Blockage on the Drag Coefficient. 


\section{4: Summary and Comparisons}

Since these tests have focused on the non-dimensional drag coefficient, the results from the Langley Full Scale Tunnel (LFST) can be easily compared to the lower speed tests in the WVU Closed Loop Wind Tunnel (WVUCLT). Though the pressure data can be insightful as to whether the separation location has moved, the main purpose of this testing was to reduce the aerodynamic drag. Thus, the primary focus of results is on the drag coefficient change relative to the baseline configuration (relative drag difference).

When looking at the results for the $1 / 2$-inch vortex generators it is evident that the height is too large for this application since all of the configurations tested increased the drag coefficient. The results for the large VG in the LFST testing of Phase II show a smaller increase in drag than that of Phase I. This is due to the alterations made to the motorcycle model. The lower fairing of the motorcycle was stiffened by adding another horizontal support to the frame that attached to the fairing. At this time the simulated radiator was also removed, thus decreasing the baseline drag, and slightly altering the airflow through the model. In the WVU tests the drag for Configuration 5 is less than the drag on Configuration 4, however in the Langley tests Configuration 5 has a marginally higher drag. In addition to the previously mentioned alterations to the model this can partially have been caused by the wall interference present in the highly blocked WVU tests.

In Phase III Configurations 1 and 2 for the 1/2-inch were re-tested in the WVUCLT. The results were noticeable different than the Phase I results, but the same changes to the model were present in the Phase III testing compared to the Phase I tests, which alters the drag coefficient. The baseline drag coefficient changed from 1.140 to 
0.618 from the stiffened fairing of the model. The changes to the model do not explain that the Phase III Configuration 1 has the higher drag coefficient and in Phase I it is Configuration 2 that has a higher drag. This could be due to the inability to repeat the drag coefficient, precisely. As shown from the standard deviations of the data collected there is some randomness in the signal in the WVU testing that is caused by the turbulence in the wind tunnel created by the turn upstream of the test section and the model itself.

Phase I testing for the 1/4-inch vortex generator resulted in several configurations that showed relative reductions in the drag coefficient. Configurations 1 and 2 displayed no measurable change from the baseline drag coefficient. Configuration 3 showed the largest drag reduction, with $\Delta \mathrm{C}_{\mathrm{D}}=0.039$. Configurations 4 and 5 also showed a reduction of approximately 30 drag counts. Phase II results for the 1/4-inch do not show any change in the drag coefficient; all the increases/reductions fall within the instrumentation error and therefore are essentially insignificant. For Phase III Configurations 3 and 4 were re-tested for the 1/4-inch VG. The drag reduction measured during Phase I was a drag increase in Phase III. Again, the changes to the model were the only difference between the two sets of tests.

For the 1/8-inch vortex generator, Phase I testing resulted in all five configurations showing a drag reduction of 30 drag counts or more. However, when Configurations 4 and 5 were tested during Phase II the results were undetectable because they were within the error of the instrumentation. Configuration 5 was also tested in Phase III where a drag increase was determined. Again the only difference between Phase I and Phase III tests are the stiffened fairing and the removal of the radiator 
simulation. The first modified configuration from Phase I was also repeated in Phase III, and similar results were found, where the drag reduction is not the same between Phase I and Phase III.

In the WVUCLT tests a relative drag reduction was measured for all of the upper fairing parametric configurations. However, the reductions measured for Configurations 4 and 5 in Phase I were not measured in the repeat tests of Phase III. Therefore, the removal of the simulated radiator and stiffening of the lower fairing had unseen effects on the airflow. Despite the differences in the model it is important to note that configurations were found that produced a relative drag reduction. The blocked test section the flow was accelerated around the model more than in an unblocked (real) flow. This over acceleration of the flow results in a smaller boundary layer height than normal. Phase II tests in the LFST were unable to show a reduction for the configurations tested. It is believed that with more testing time in the unblocked scenario configurations could be found to reduce the drag. This configuration would most likely contain vortex generators closer to the leading edge than the configurations tested in the LFST. 


\subsection{Conclusions and Recommendations}

This chapter discusses the conclusions drawn from the results of the three phases of wind tunnel testing on the Tul-Aris motorcycle model. Also discussed are recommended future steps to further the understanding of the use of vortex generators in this type of application.

\subsection{Conclusions}

The purpose of this research was to determine the feasibility of using vortex generators to decrease the aerodynamic drag on a racing motorcycle. From the results of Phase I it is evident that the use of vortex generators has the ability to reduce the drag on a motorcycle. There is a level of randomness to measurements taken during Phase I; this is speculated to be due to the upstream turn in the wind tunnel and the vibration in the lower fairing of the model. However, this error is accounted for in the standard deviation.

Phase II tests in the ODU LFST showed no significant change in the drag coefficient which leads to the conclusion that the tunnel blockage ratio has a large effect on performance the vortex generators. The changes to the model, removal of the radiator simulator and the stiffened lower fairing, also had an effect on the drag coefficient. But these changes have similar effects on all tests, including the baseline; therefore the impact of the changes to the model are negligible for the relative drag values.

Phase III testing was conducted in the WVU Closed Loop Wind Tunnel with the altered model. Of the configurations that were tested in both Phase I and Phase III, discrepancies are present between the two data sets. The cause of the disagreement is 
most likely the alterations that were made to the model between the Phase I and Phase III tests. The parametric studies of the upper and lower fairing show that there is an optimal position of the vortex generators in the WVU Closed Loop Wind Tunnel that is between the leading edge and the center of the fairing. It is expected that this optimal location(s) may be different at higher (racing) speeds.

Comparing Phase III to Phase II results, the interference of test section blockage on the effectiveness of the VG's was significant. There were also tests conducted with an increase in the blockage of the wind tunnel which drastically changed the drag coefficient. This led to the conclusion that as the blockage ratio of the test increases the uncertainty of the measured drag coefficient increases. This uncertainty is not based on the standard deviation of the measurement but the decrease in the likelihood of a realistic simulation of the measurement that would occur in an unobstructed free air flow. In comparing the baseline drag coefficient for Phase II and Phase III of testing it was determined that neither method of adjusting for such a high blockage ratio adequately simulates the unblocked case. The Phase II, unblocked baseline drag coefficient $\left(\mathrm{C}_{\mathrm{D}-}\right.$ Baseline $=0.506)$ is less than the adjusted velocity $\left(C_{D-B a s e l i n e}=0.614\right)$ and the Barlow, Rae and Pope $\left(\mathrm{C}_{\mathrm{D}-\mathrm{Baseline}}=0.704\right)$ method of accounting for test section blockage.

Despite the inability to show a drag reduction in the Langley Full Scale Wind Tunnel tests it is believed that given adequate testing time a VG configuration could be found that would reduce the drag on the model. A configuration with the vortex generators closer to the leading edge of the model is suggested as a possibility for this optimum configuration. This belief is based upon the ability to achieve relative drag reduction in both phases of testing in the WVU tests. Despite the effects of test section 
blockage, the $1 / 8$-inch vortex generator was able to provide a repeatable relative drag reduction compared to the baseline cases for both Phase I and III. Differences between the two sets of WVU tests are most likely due to the placement and re-placement of the vortex generators. Despite every effort to make the VG placement identical it was virtually impossible to duplicate the exact placement with respect to the angle of incidence as well as being precisely in the same location. It is estimated that the placement of the VG's were within $\pm 1 / 8$-inch of the original location, and that the angle of incidence varied by \pm 5 degrees. Another source of error is the inconsistency in the thickness of the glue used in the VG application process.

In summary, the testing at WVU showed a drag reduction for several VG configurations. However, the testing at the LFST could not verify this reduction in an unblocked flow. Thus the configurations that worked in the WVU tests are not expected to work in a real world scenario, but they do show that it is possible to find some configurations that can reduce the drag. Thus, testing of different configurations and sizes of vortex generators would produce a reduction of drag in an unblocked wind tunnel and that would directly relate to a real world drag reduction.

\subsection{Recommendations}

Further testing on this or similar applications of vortex generators should be conducted in a situation that better simulates the real world. This would start with the use of a larger wind tunnel facility that would have a blockage ratio of $5 \%$ or less based on the $5.298 \mathrm{ft}^{2}$ frontal area model. Avoiding the blockage effects could also be done by 
using a scale model of the motorcycle or conducting tests in a real world scenario on a closed course, such as coast down testing for drag.

It is also of interest to investigate the effect of testing with a non-rotating tire on the drag coefficient. Another thing that should be studied before applying vortex generators on an actual racing motorcycle would include the effects of tilting the motorcycle while turning. The effects of the vortices created by the VG's on a motorcycle behind and to the side of the motorcycle also need to be researched to avoid any undesired racing effects. Exposure to the randomness of real air with wind gusts from various directions may also lead to undesirable results so some investigation should be conducted into how gusts from various directions affect the performance of the vortex generators.

The use of a Computational Fluid Dynamics (CFD) simulation could also be used investigate the airflow over a racing motorcycle. CFD solutions would cost less than full scale testing and would provide an opportunity to simulate full racing speeds ( $185 \mathrm{mph})$. A computer simulation could also reproduce the tire rotation as well as a simulation of the radiator.

It may be beneficial to use another type of flow control device, such as dimple tape or a boundary layer tripping wire. Combinations of these all of these devices (including the vortex generators tested in this research) could provide better results than one individual device alone. The use of a suction device may also be attempted to determine the amount of power that would be required. Suction could be passively created through the use of a venturi or ejector. 


\subsection{Vita}

Gerald Martin Angle II, the only son of Mr. And Mrs. Gerald Angle, was born in Hagerstown, Maryland, on June 4, 1978. He was educated in the Washington County, Maryland, Public School System, and graduated from Boonsboro High School in May 1996. He received two Bachelor of Science Degrees from West Virginia University in May 2001 with majors in Aerospace and Mechanical Engineering.

In August 2001, he entered the graduate program at West Virginia University and is a candidate for the Master of Science Degree in Aerospace Engineering in December 2002. 


\subsection{References}

Ashill, P. R., Fulker, J. L. and Hackett, K. C., "Research at DERA on Sub Boundary Layer Vortex Generators (SBVGs)," AIAA Paper 2001-0887, January 2001.

Barlow, J. B., Rae Jr., W. H., Pope, A., Low-Speed Wind Tunnel Testing $3^{\text {rd }}$ Edition, John Wiley \& Sons, Inc., New York, 1999.

Beauvais, F. N., Tignor, S. C., Turner, T. R., "Problems of Ground Simulation in Automotive Aerodynamics," SAE/PT-78/16, 1978.

Beckwith, T. G., Marangoni, R. D., Lienhard V, J. H., Mechanical Measurements Fifth Edition, Addison-Wesley Publishing Co., Reading, Mass., 1995.

Carr, G. W., Eckert, W., "A Further Evaluation of the Ground-Plane Suction Method for Ground Simulation in Automotive Wind Tunnels," SAE Paper 940418, 1994.

Cogotti, A., "Aerodynamic characteristics of car wheels. Impact of aerodynamics on vehicle design,” Int. J. of Vehicle Design. SP3, London, 1983.

Dryden, H. L., Abbott, I. H., The Design of Low-Turbulence Wind Tunnels. NACA Rep. 940, 1949.

Fabijanic, J., George, A. R., "An Experimental Investigation of the Aerodynamics of Automobile Wheel Wells,” AIAA Paper 96-2475-CP, 1996.

Fackrell, J.E. and Harvey, J.K., "The Aerodynamics of an Isolated Road Wheel," Proceedings of the $2^{\text {nd }}$ AIAA Symposium on the Aerodynamics of Sports and Competition Automobiles. Vol. 16, pp. 119-125, 1974.

Hackett, J. E., Baker, J. B., Williams, J. E., Wallis, S. B., "On the influence of ground movement and wheel rotation in tests on modern car shapes," SAE Paper 870245, 1987.

Hoerner, S. F., Fluid Dynamic Drag, Hoerner Fluid Dynamics, Brick Town, N.J., 1965.

Hucho, W. H., Aerodynamics of Road Vehicles $4^{\text {th }}$ Edition, SAE, Inc., Warrendale, Pa., 1998.

Katz, J., Walters R., "Investigation of Wind-Tunnel Wall Effects in High Blockage Testing," AIAA Paper 95-0438, March 1995.

Kraft, E.M., Dahm, W.J.A., "Direct Assessment of Wall Interference in a TwoDimensional Subsonic Wind Tunnel," AIAA Paper 82-0187, January, 1982. 
Lin, J. C., "Control of Turbulent Boundary-Layer Separation using Micro-Vortex Generators," AIAA Paper 99-3404, July 1999.

Mercker, E., Breuer, N., Berneburg, H., Emmelmann, H. J., "On the Aerodynamic Interference Due to the Rolling Wheels of Passenger Cars," SAE Paper 910311, 1991.

Mercker, E., Knape, H.W., "Ground simulation with moving belt and tangential blowing for full-scale automotive testing in a wind tunnel," SAE Paper 890367, 1989.

Mercker, E., Wiedemann, J., "Comparison of Different Ground Simulation Techniques for Use in Automotive Wind Tunnels," SAE Paper 900321, 1990.

Patel, M. P., Carver, R., Lisy, F. J., Prince, T. S. and Ng, T., "Detection and Control of Flow Separation Using Pressure Sensors and Micro-Vortex Generators," AIAA Paper 2002-0268, January 2002.

Scheiman, J., "Comparison of Experimental and Theoretical Turbulence Reduction Characteristics for Screens, Honeycomb, and Honeycomb-Screen Combinations," NASA TP 1958, December, 1981.

Scheiman, J., "Considerations for the Installation of Honeycomb and Screens To Reduce Wind-Tunnel Turbulence,” NASA TM 81868, August, 1981.

Schubauer, G. B., Spangenberg, W. G., Klebanoff, P.S., “Aerodynamic Characteristics of Damping Screens," NACA T.N. 2001, 1950.

Smith, G. R., Bond, R. E., Loth, J. L., Morris, G. J., "NASP Take-Off Lift Loss Alleviation," NASA/WVU/NASP No. 796-1, 1997.

Stapleford, W. R. and Carr, G. W., "Aerodynamic Characteristics of Exposed Rotating Wheels,” MIRA Report No. 1970/2, 1970.

Tai, T. C., "Effect of Micro-Vortex Generators on V-22 Aircraft Forward-Flight Aerodynamics," AIAA Paper 2002-0553, January 2002.

Tuluie, R., Ericksen, G., "Racing Motorcycle Design Process Using Physical and Virtual Testing Methods," SAE Paper 2000-01-3576, 2000.

Weiss, H., "Estimating Experimental Errors," Grumman Aircraft Engineering Corp, Calverton, N.Y.

Wendt, B. J. and Hingst, W.R., "Flow Structure in the Wake of a Wishbone Vortex Generator," AIAA Journal, Vol. 32, Nov. 1994, pp. 2234-2240. 
Wendt, B. J., Reichert, B. A., "Spanwise Spacing Effects on the Initial Structure and Decay of Axial Vortices," AIAA Paper 96-2518-CP, 1996.

Westphal, R. V., Pauley, W.R., and Eaton, J.K., "Interaction Between a Vortex and a Turbulent Boundary Layer-Part 1: Mean Flow Evolution and Turbulence Properties," NASA TM 88361, Jan. 1987.

Wetzel, K. K., Farokhi, S., "Interaction of Riblets and Vortex Generators on an Airfoil," AIAA Paper 96-2428-CP, 1996.

Wheeler, G. O., “Low Drag Vortex Generators,” United States Patent 5058837, 1991.

Wheeler, G. O., "Means for Maintaining Attached Flow of a Flowing Medium," United States Patent 4455045, June, 1984.

Young, D. F., Munson, B. R., Okiishi, T. H., A Brief Introduction to Fluid Mechanics, John Wiley \& Sons, Inc., New York, NY, 1997.

Zhang, X., "Co- and Contra rotating Streamwise Vortices in a Turbulent Boundary Layer," Journal of Aircraft, Vol. 32, No. 5, September-October 1995, pp. 1095-1101. 


\section{Appendix A}

Additional Pressure Figures Not Shown in Results Section 


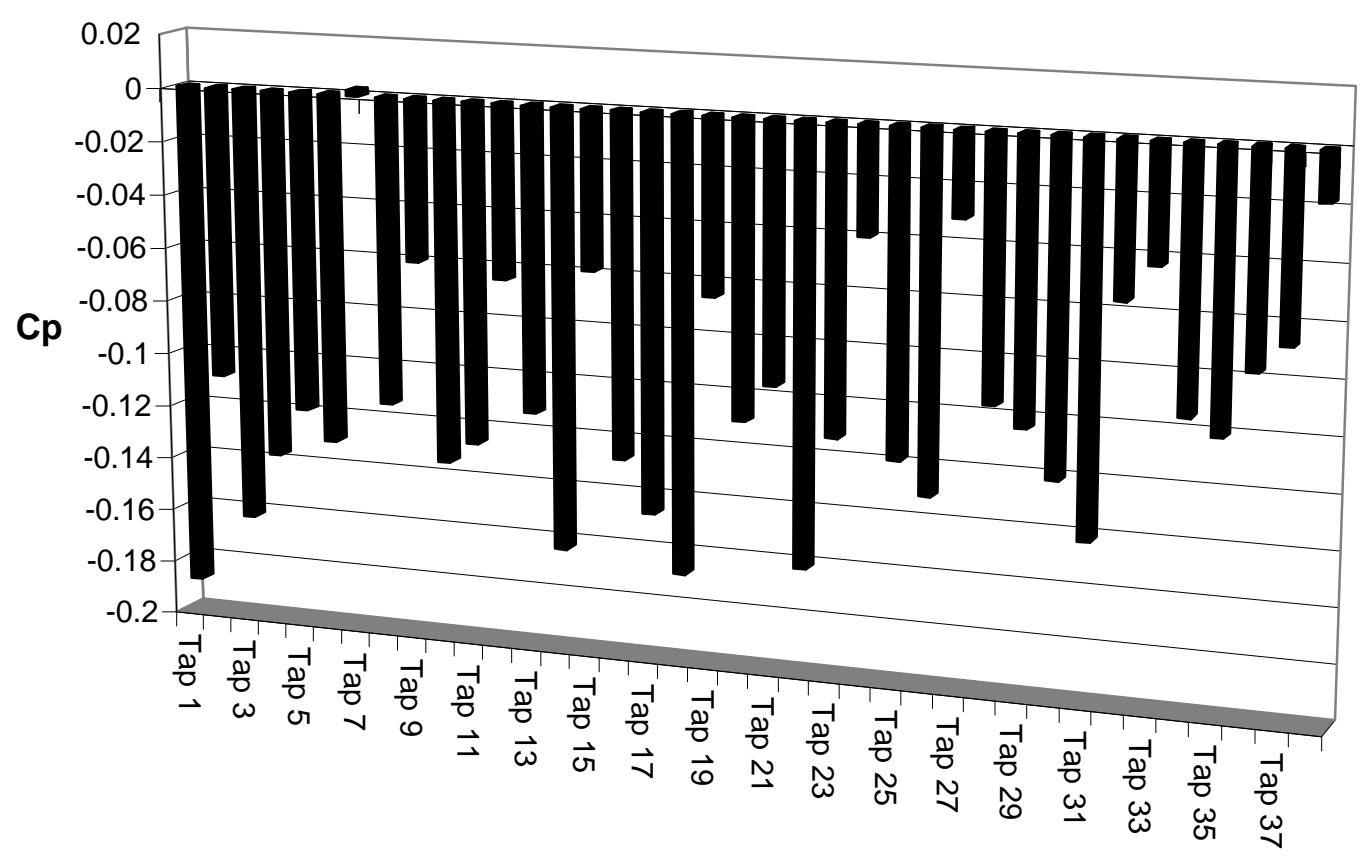

Figure A.1: Phase I Average Pressure Coefficients for Three Baseline 2 Tests.

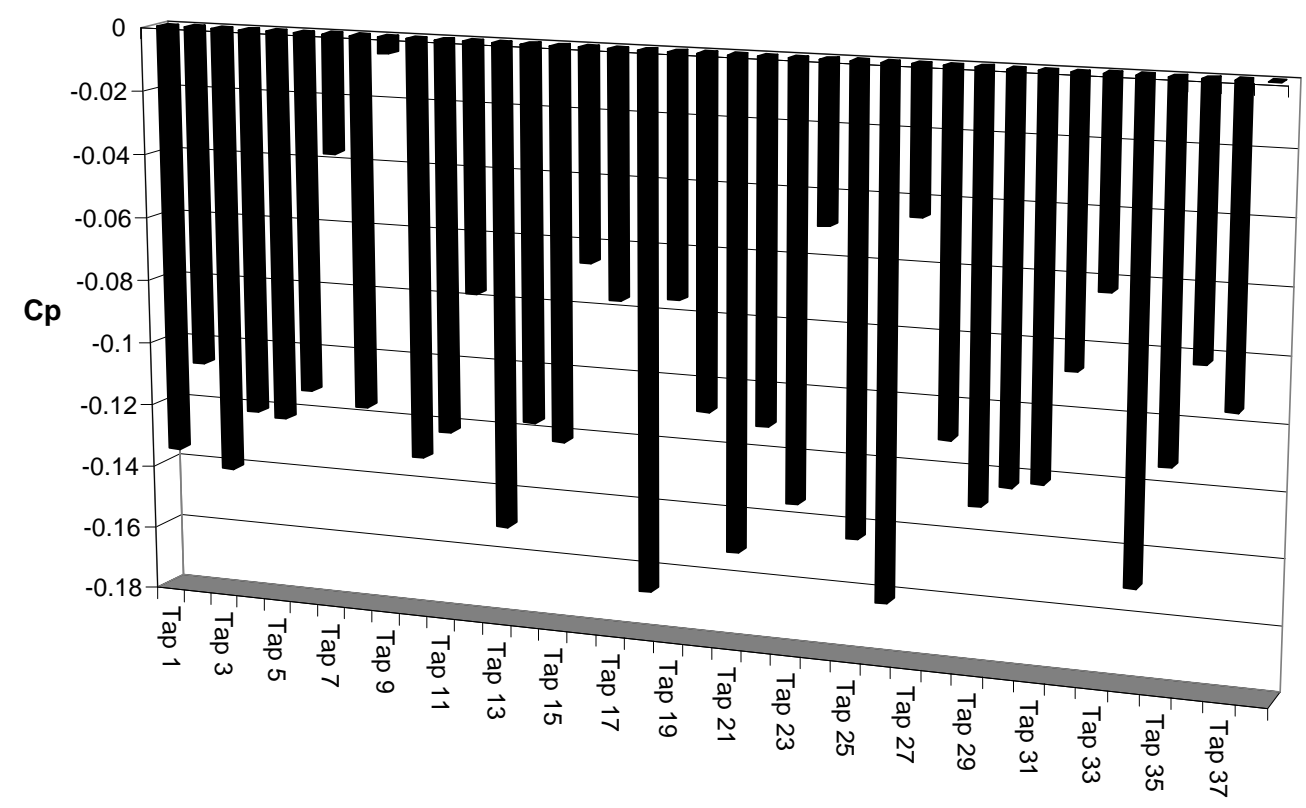

Figure A.2: Phase I Average Pressure Coefficients for Three Baseline 3 Tests. 


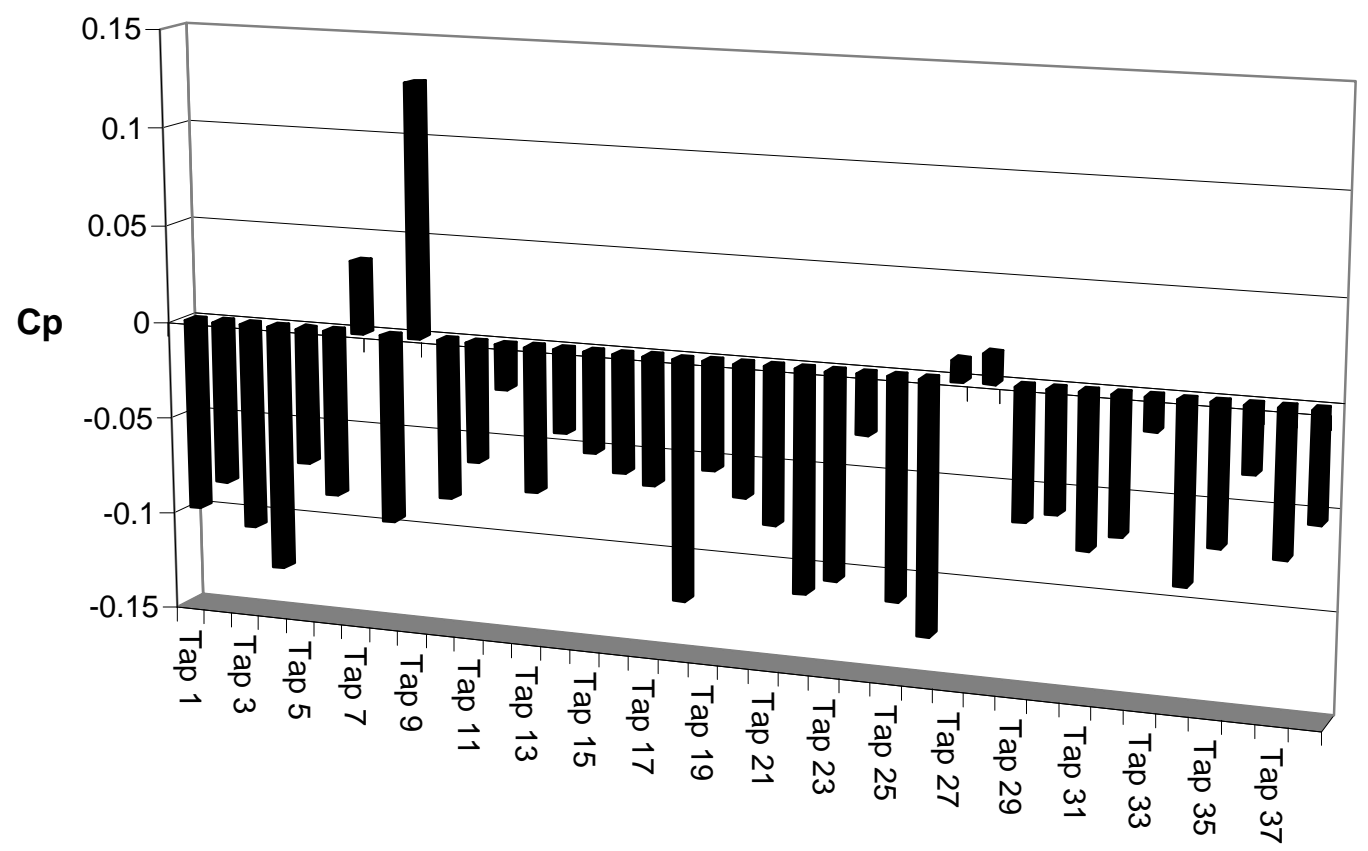

Figure A.3: Phase I Average Pressure Coefficients for Three Baseline 4 Tests.

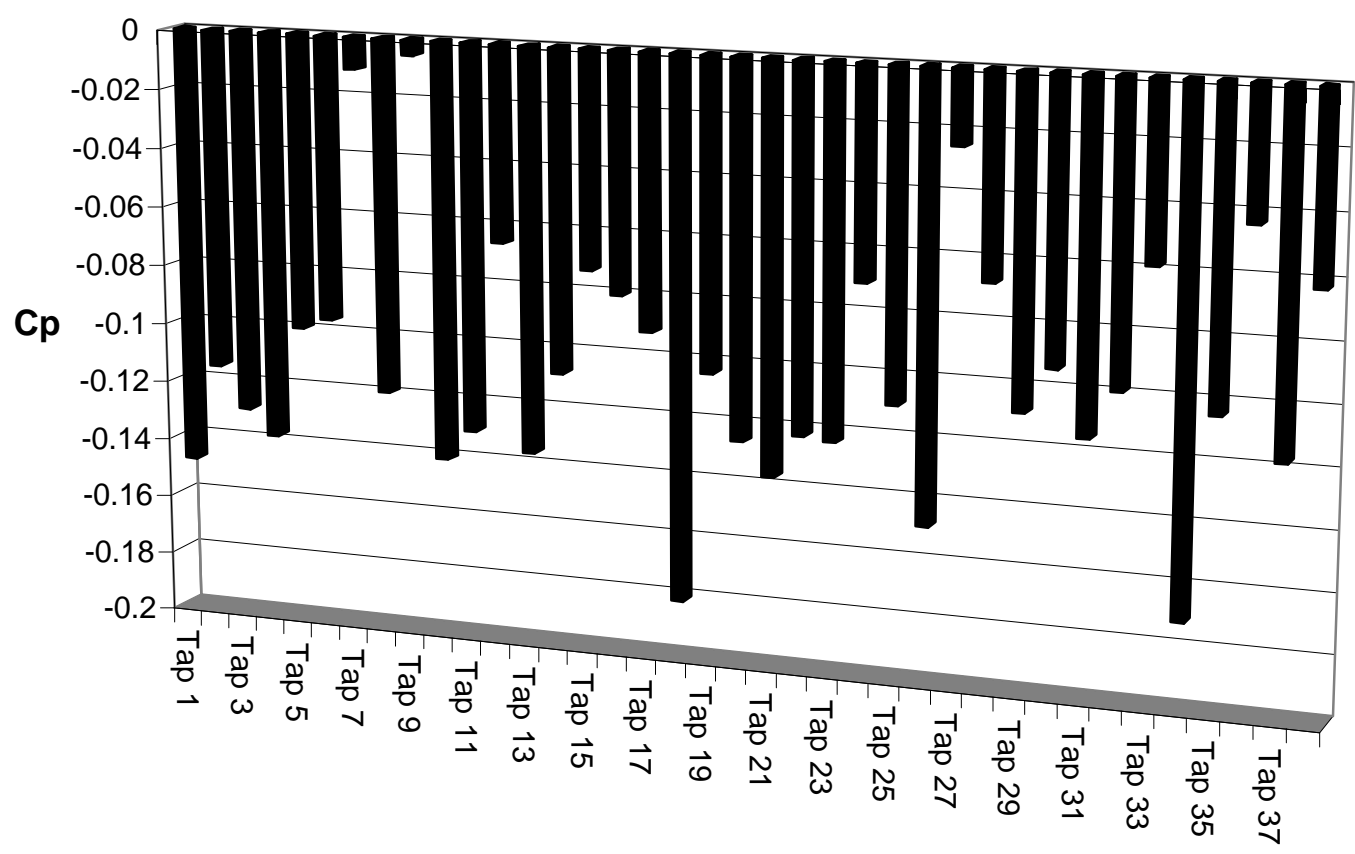

Figure A.4: Phase I Average Pressure Coefficients for Modified Baseline Test. 


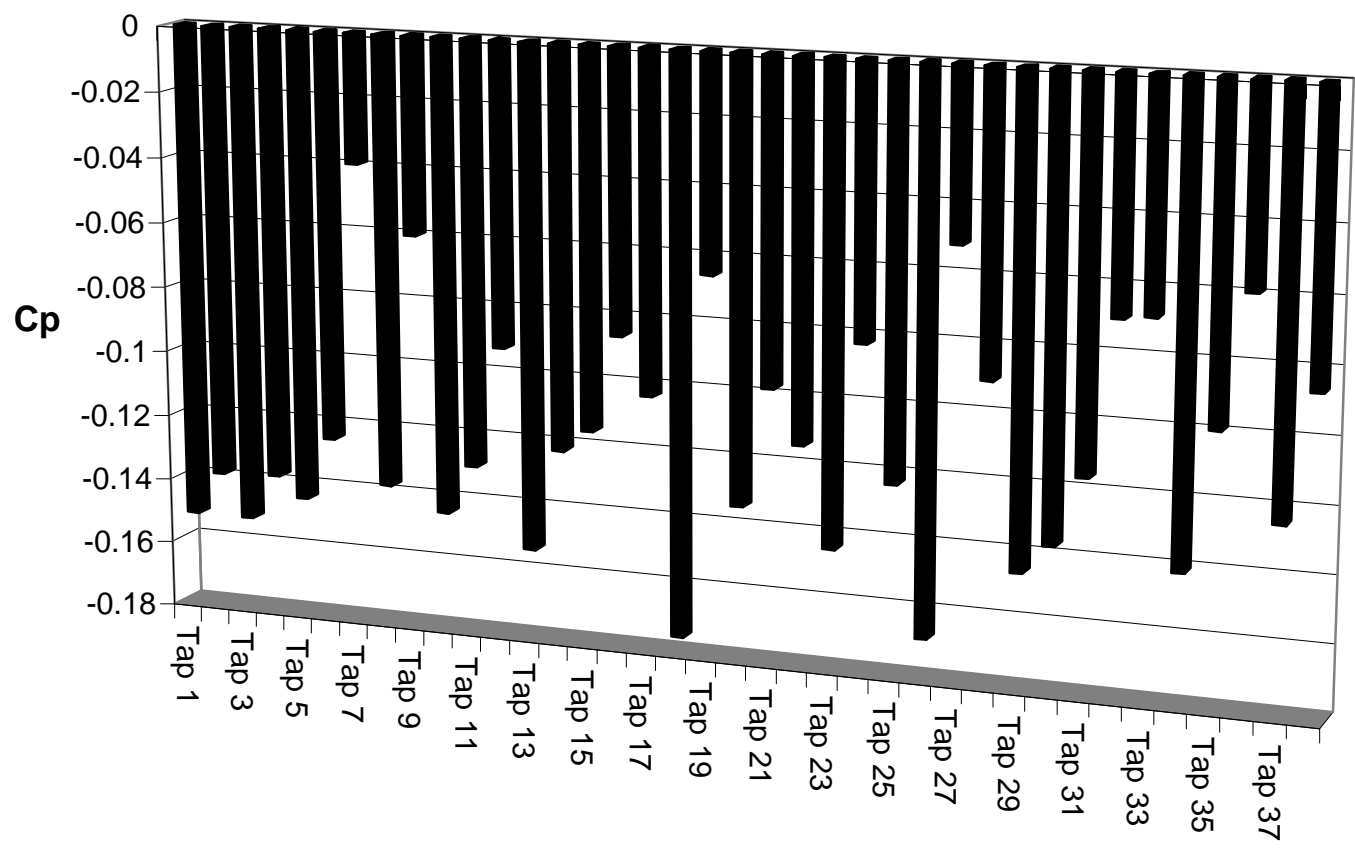

Figure A.5: Phase I Average Pressure Coefficients for Three 1/2-inch VG Configuration 2 Tests.

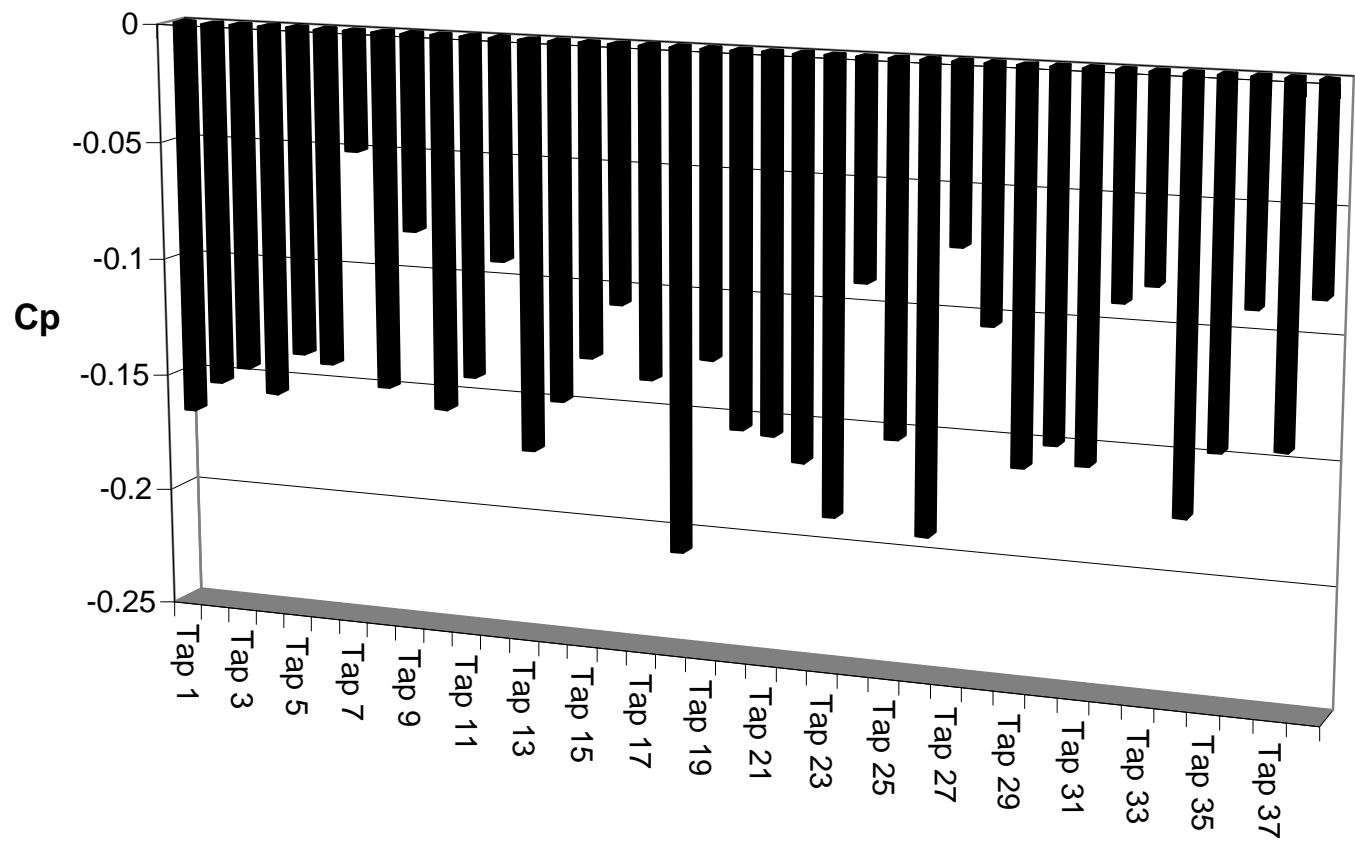

Figure A.6: Phase I Average Pressure Coefficients for Three 1/2-inch VG Configuration 3 Tests. 


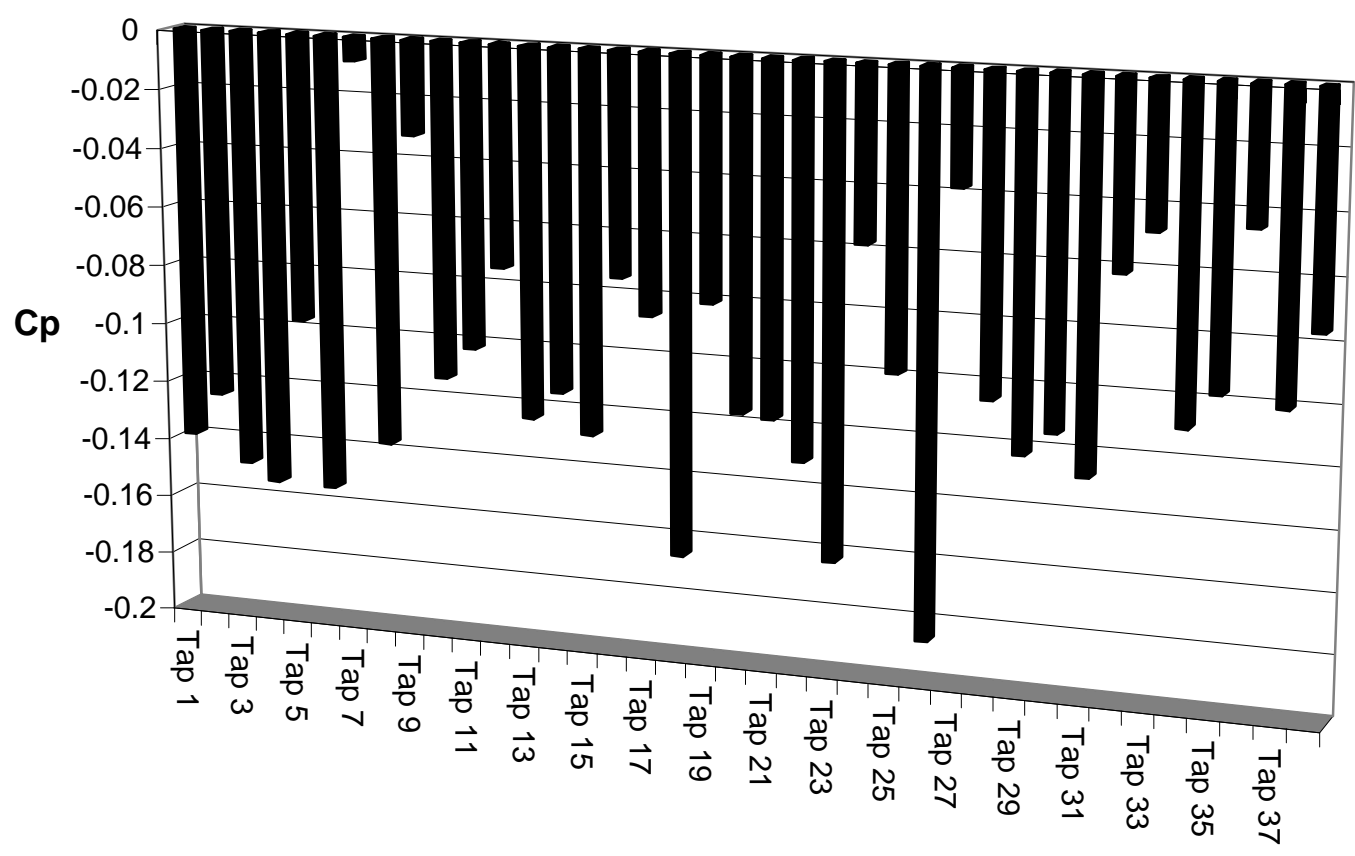

Figure A.7: Phase I Average Pressure Coefficients for Three 1/2-inch VG Configuration 4 Tests.

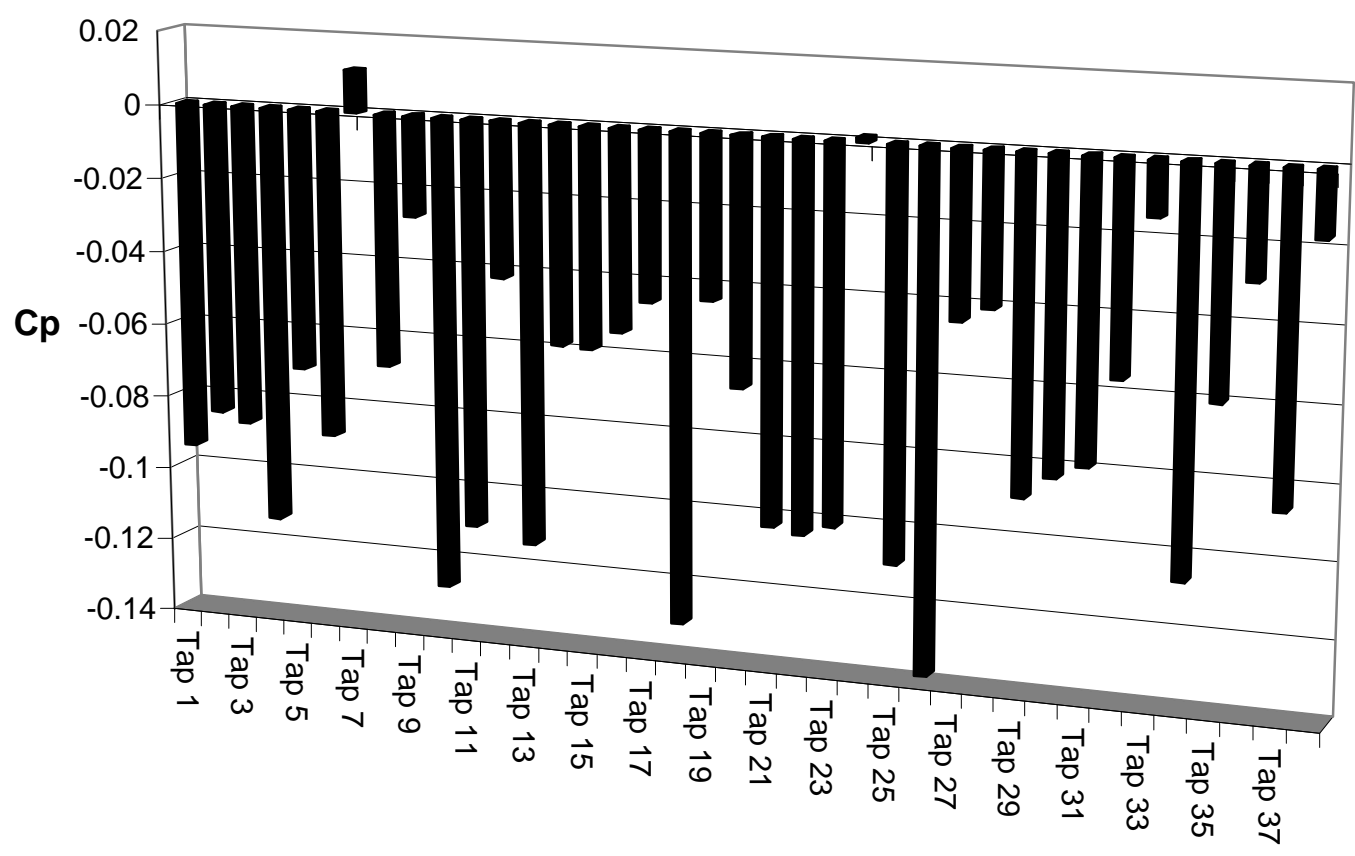

Figure A.8: Phase I Average Pressure Coefficients for Three 1/2-inch VG Configuration 5 Tests. 


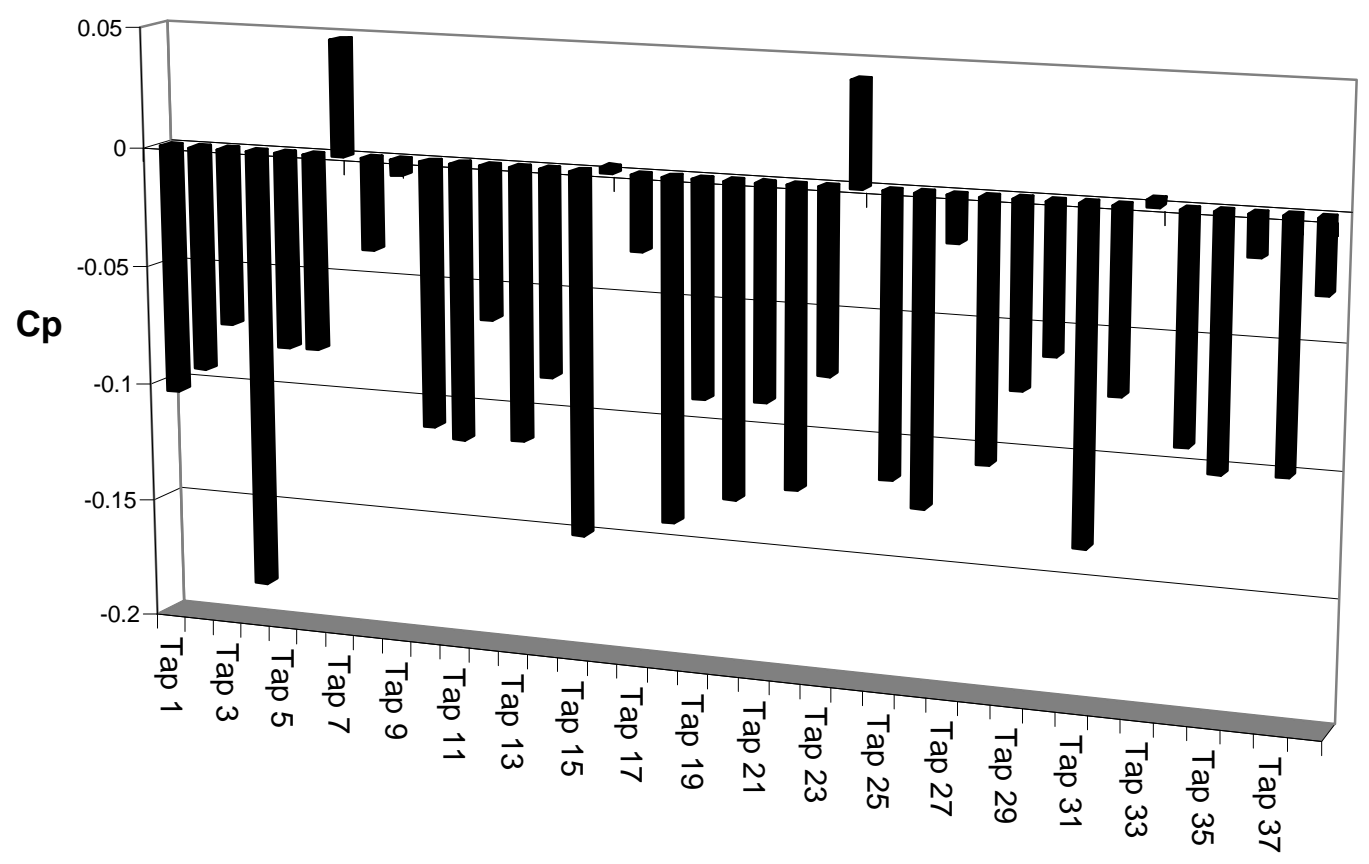

Figure A.9: Phase I Average Pressure Coefficients for Three 1/4-inch VG Configuration 1 Tests.

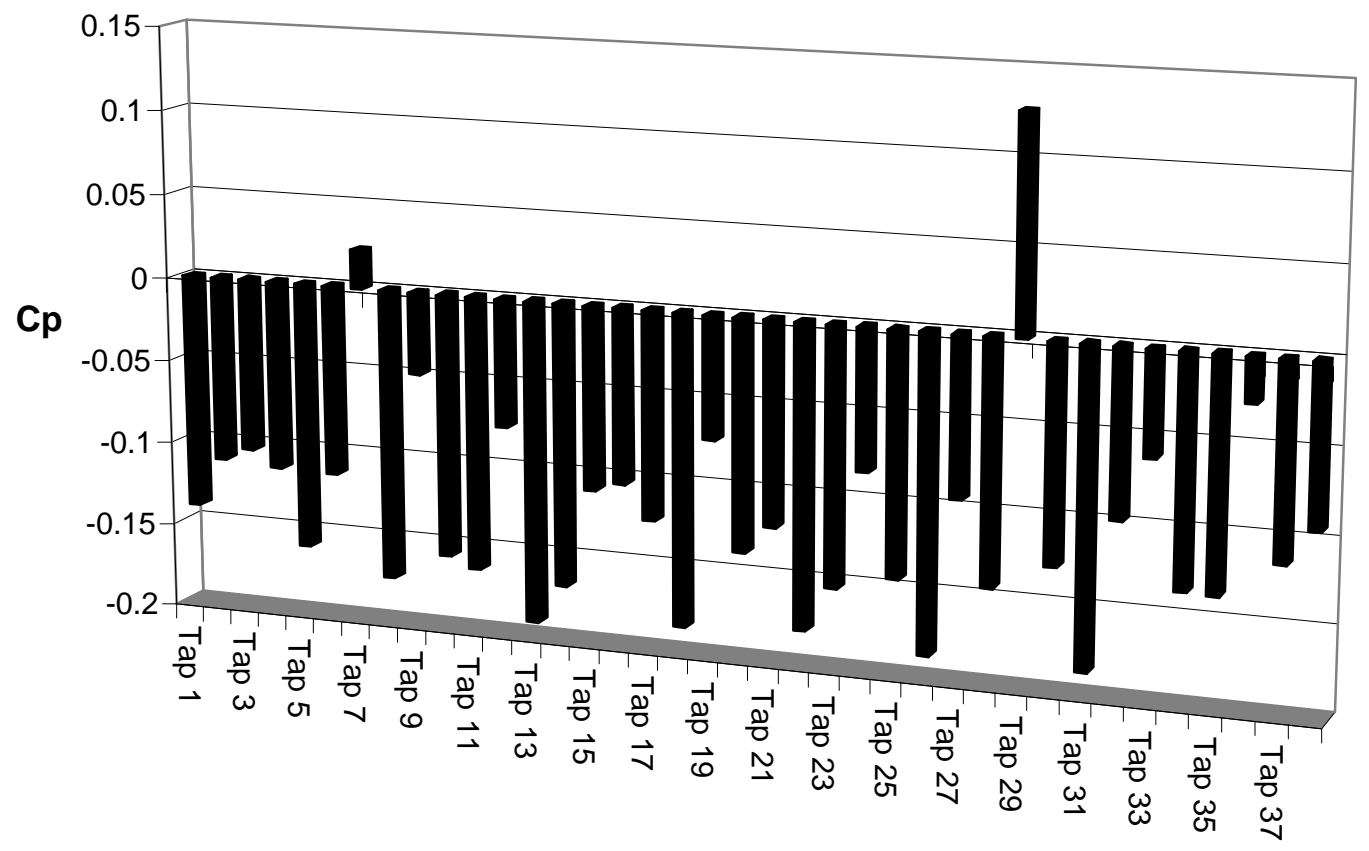

Figure A.10: Phase I Average Pressure Coefficients for Three 1/4-inch VG Configuration 3 Tests. 


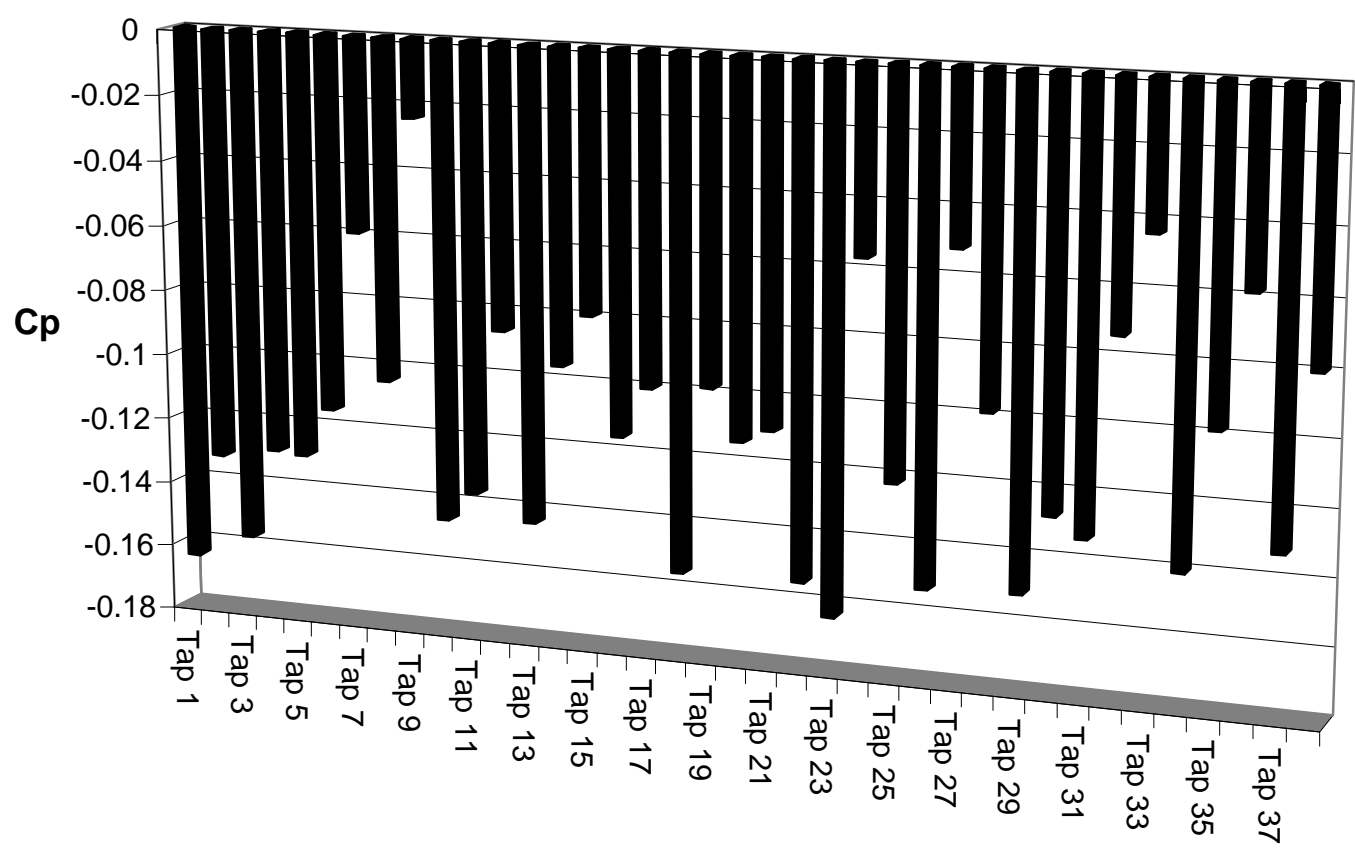

Figure A.11: Phase I Average Pressure Coefficients for Three 1/4-inch VG Configuration 4 Tests.

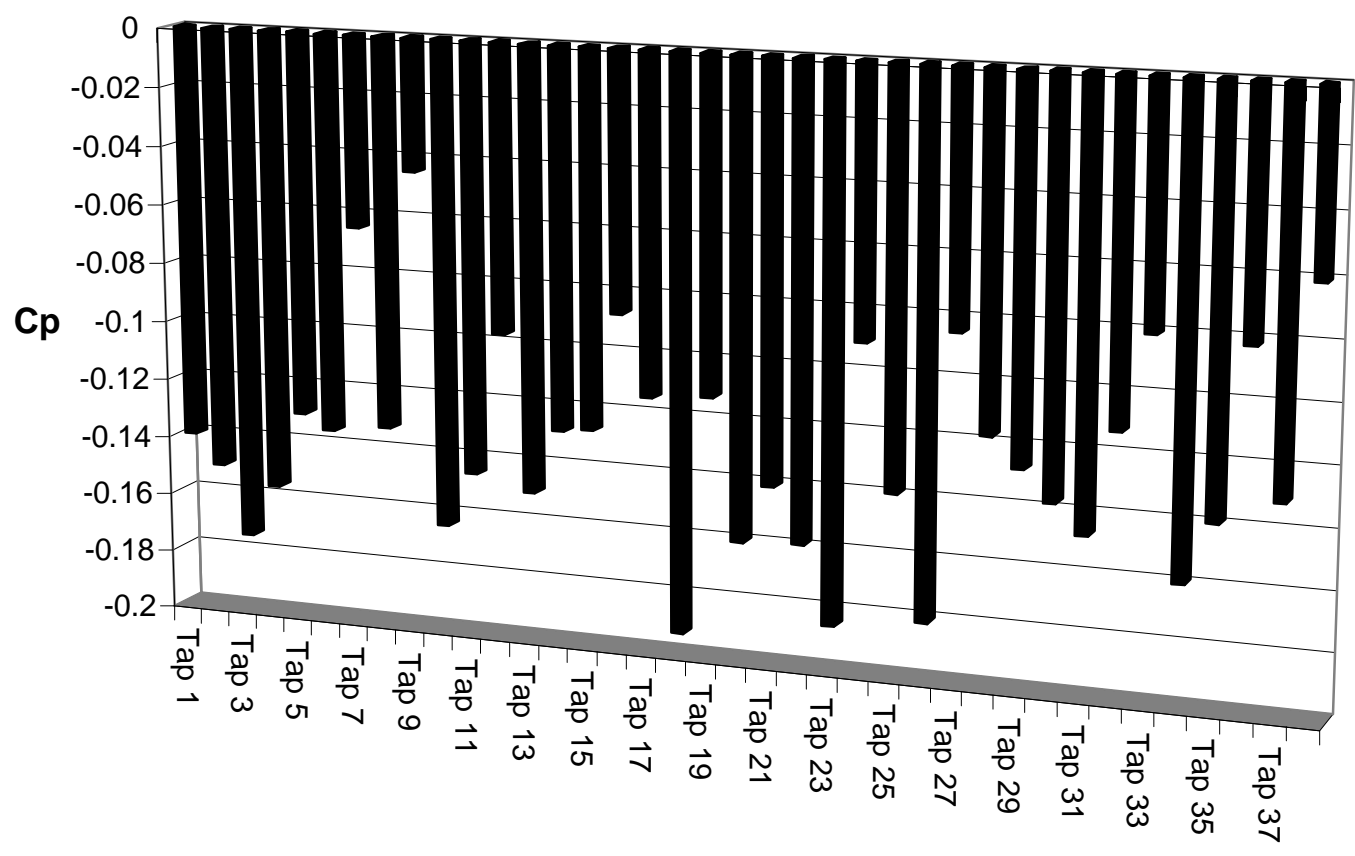

Figure A.12: Phase I Average Pressure Coefficients for Three 1/4-inch VG Configuration 5 Tests. 


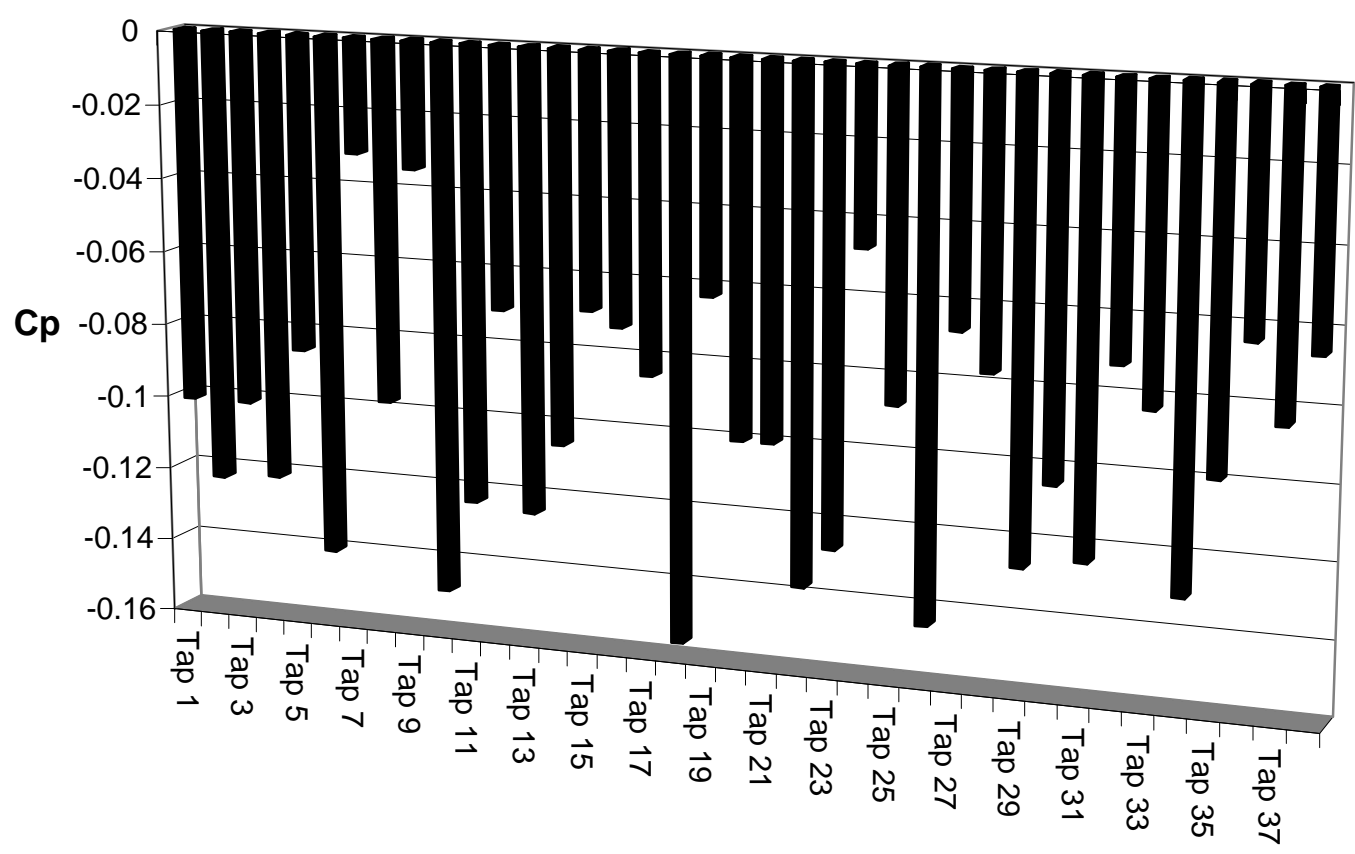

Figure A.13: Phase I Average Pressure Coefficients for Three 1/8-inch VG Configuration 1 Tests.

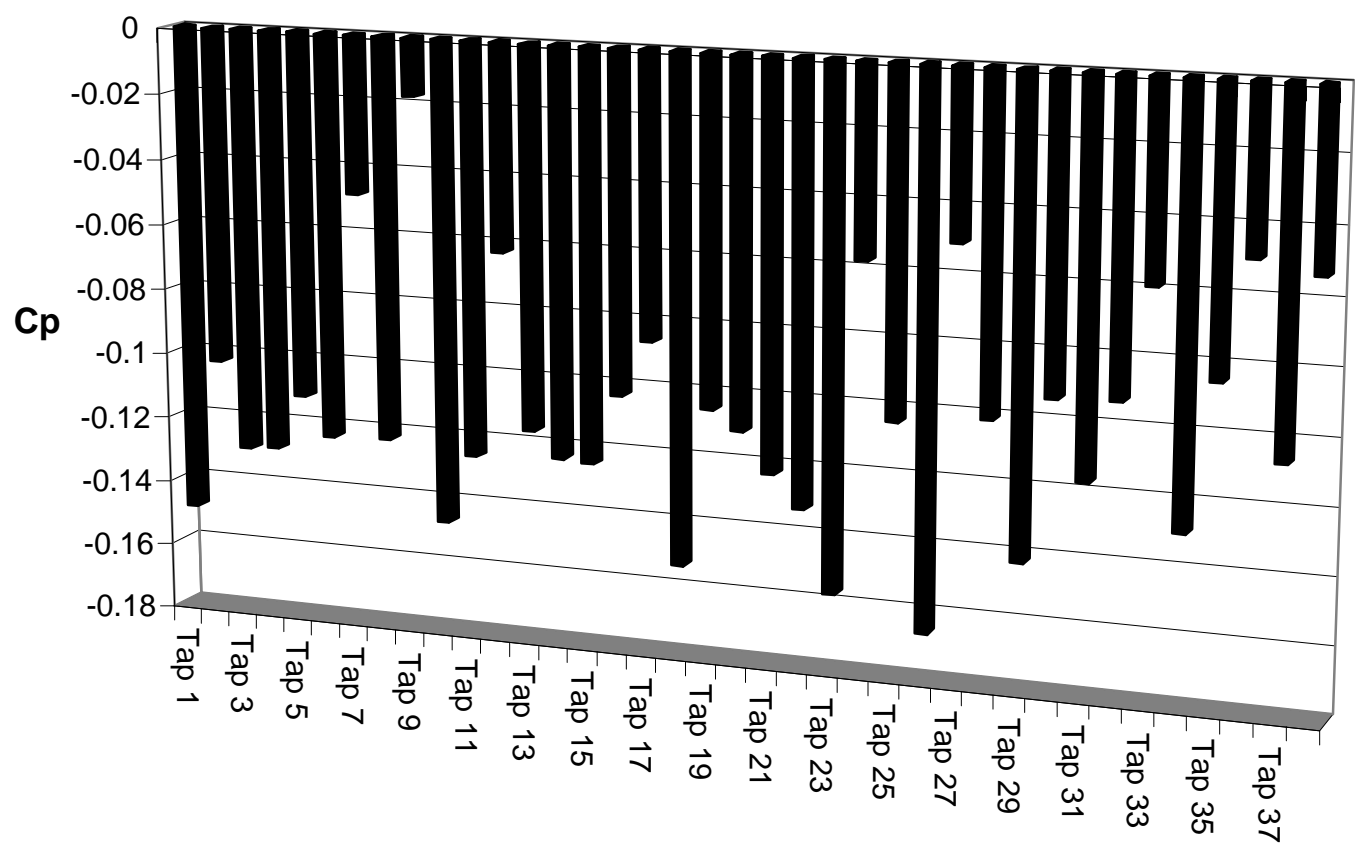

Figure A.14: Phase I Average Pressure Coefficients for Three 1/8-inch VG Configuration 2 Tests. 


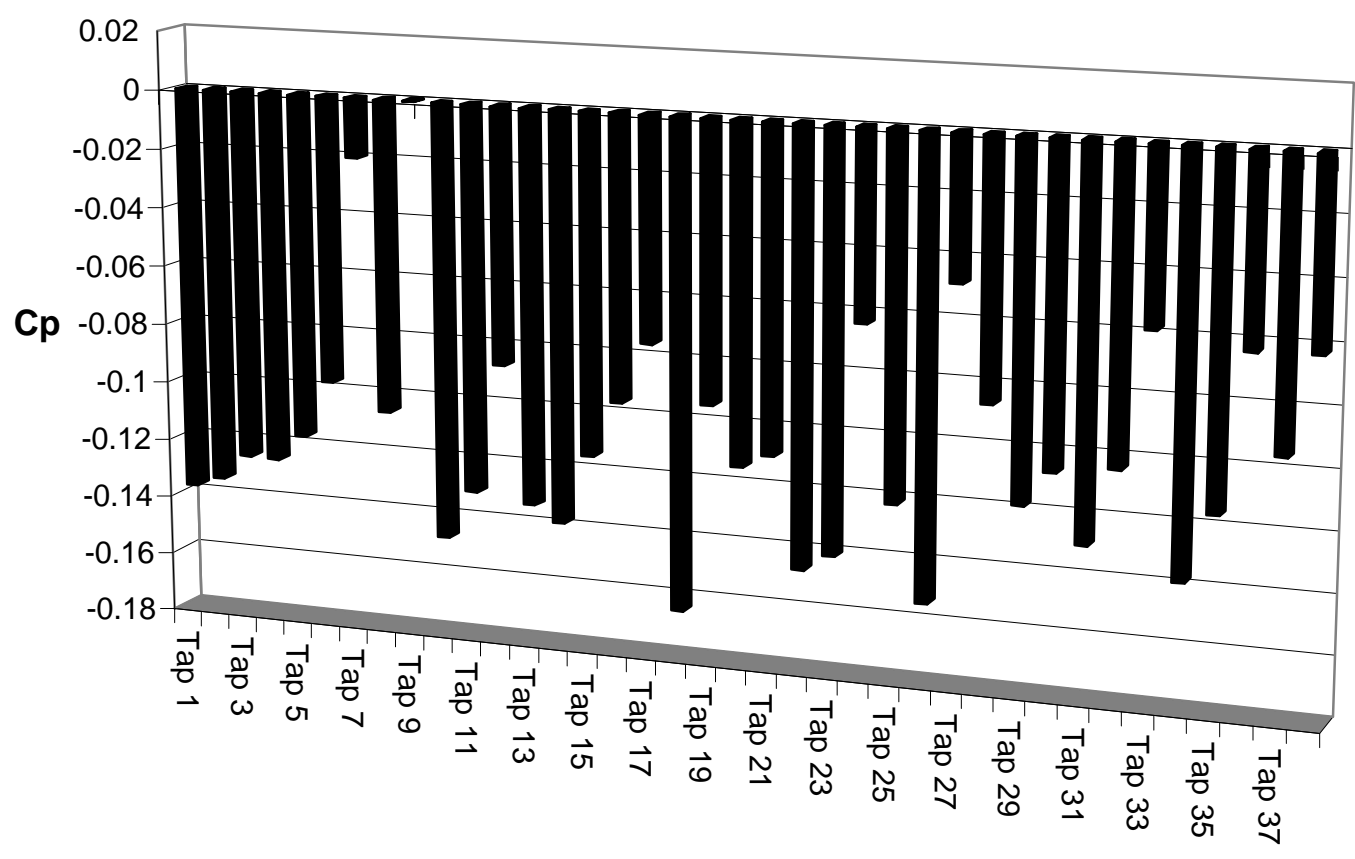

Figure A.15: Phase I Average Pressure Coefficients for Three 1/8-inch VG Configuration 4 Tests.

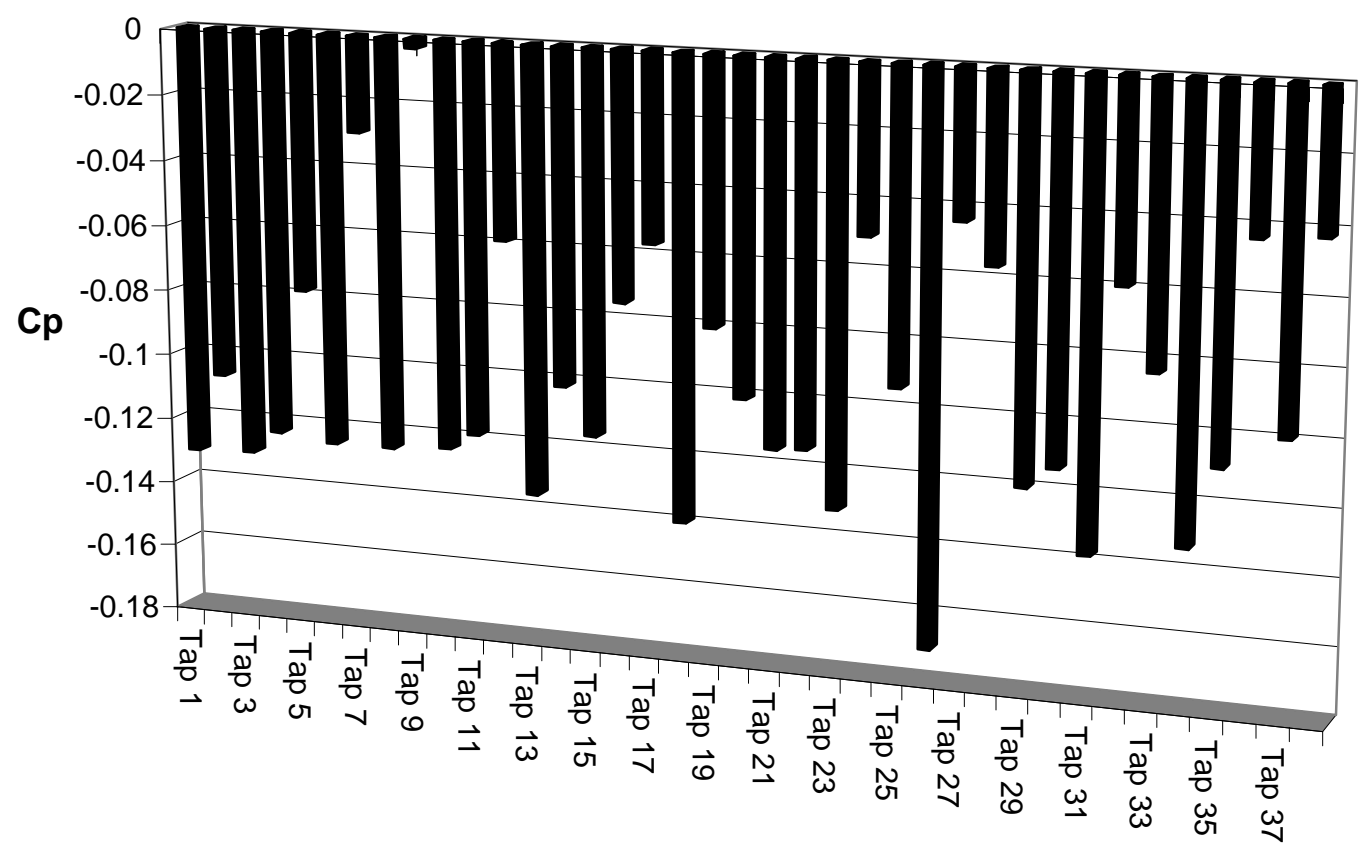

Figure A.16: Phase I Average Pressure Coefficients for Three 1/8-inch VG Configuration 5 Tests. 


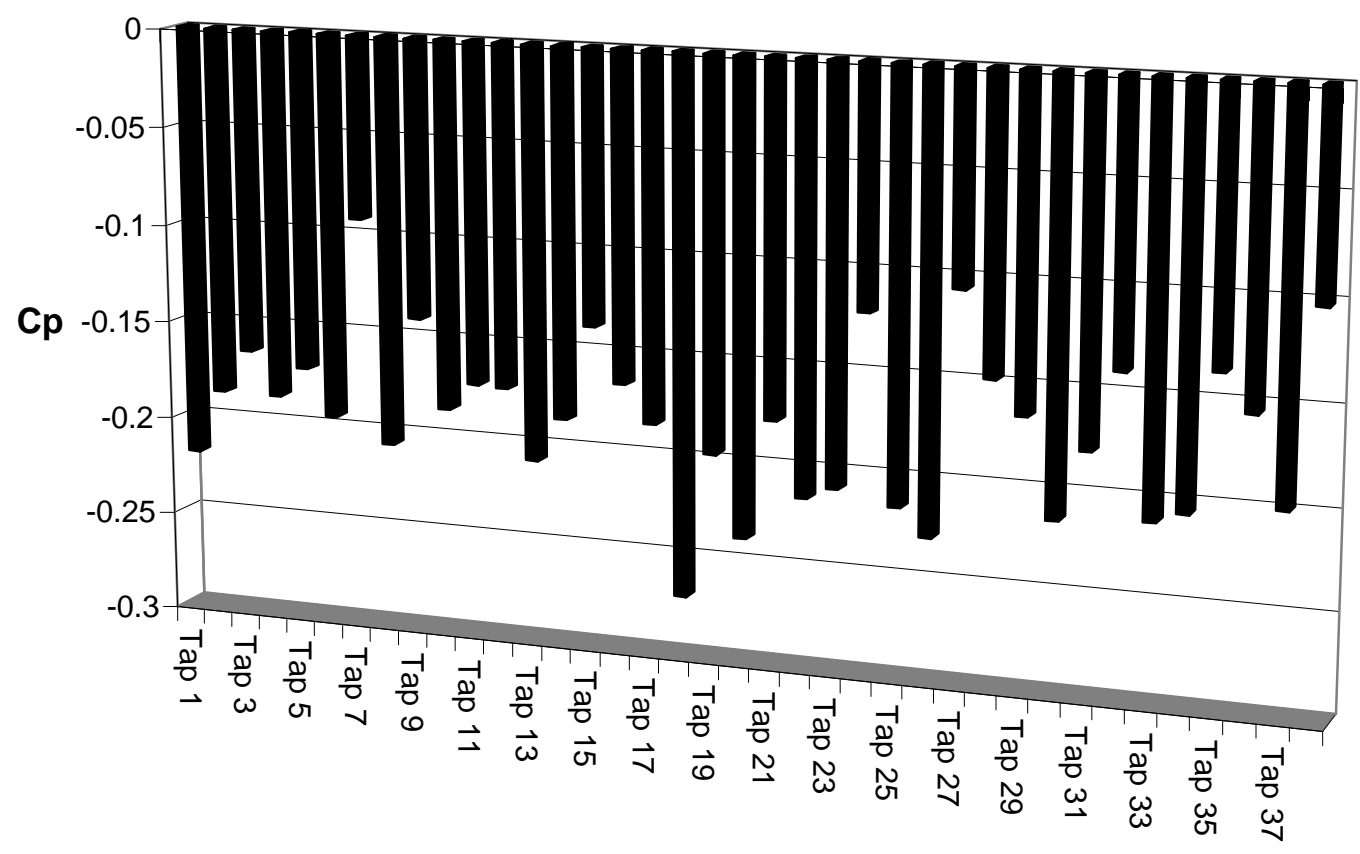

Figure A.17: Phase I Average Pressure Coefficients for 1/8-inch VG Modified Configuration 2 Test.

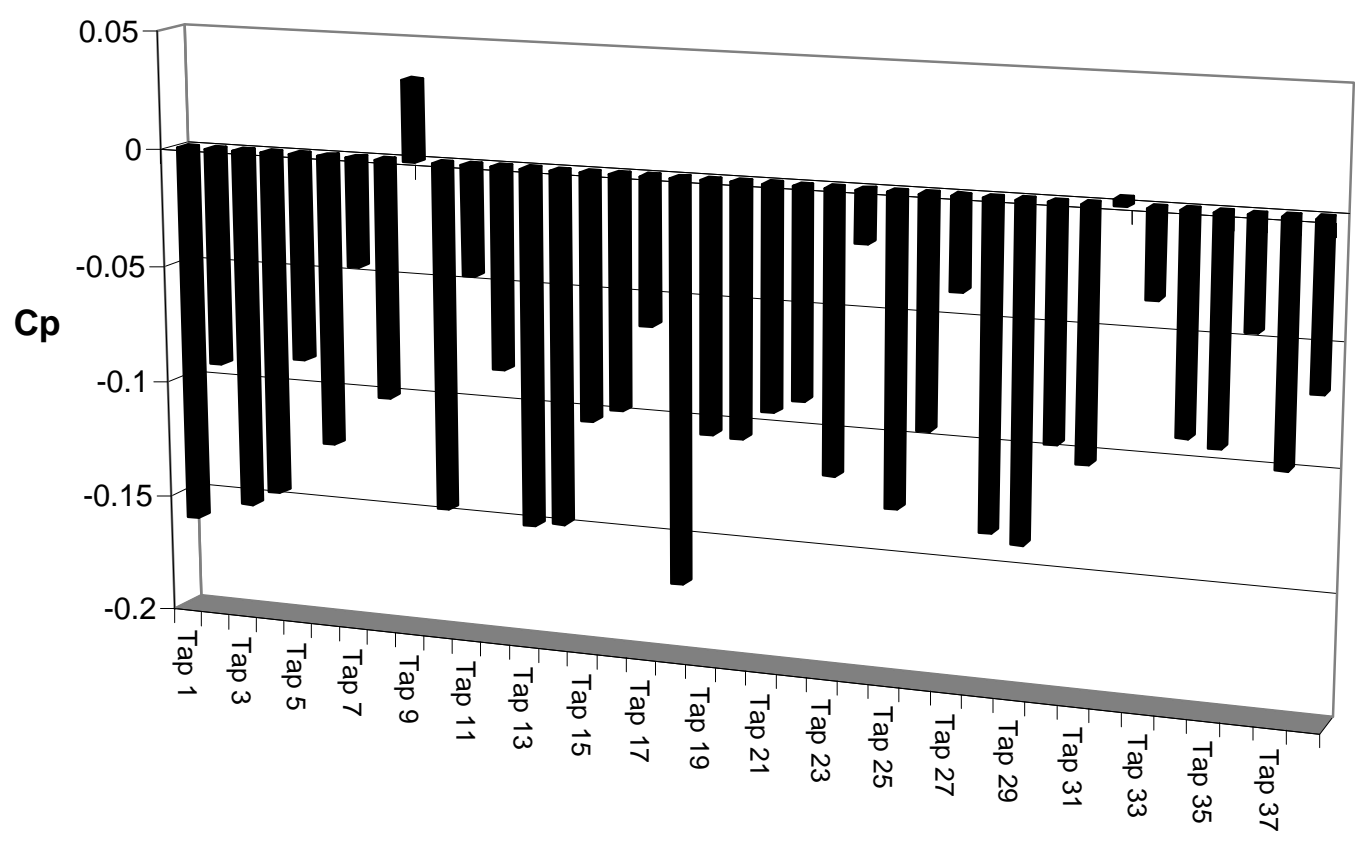

Figure A.18: Phase I Average Pressure Coefficients for 1/8-inch VG Modified Configuration 3 Test. 


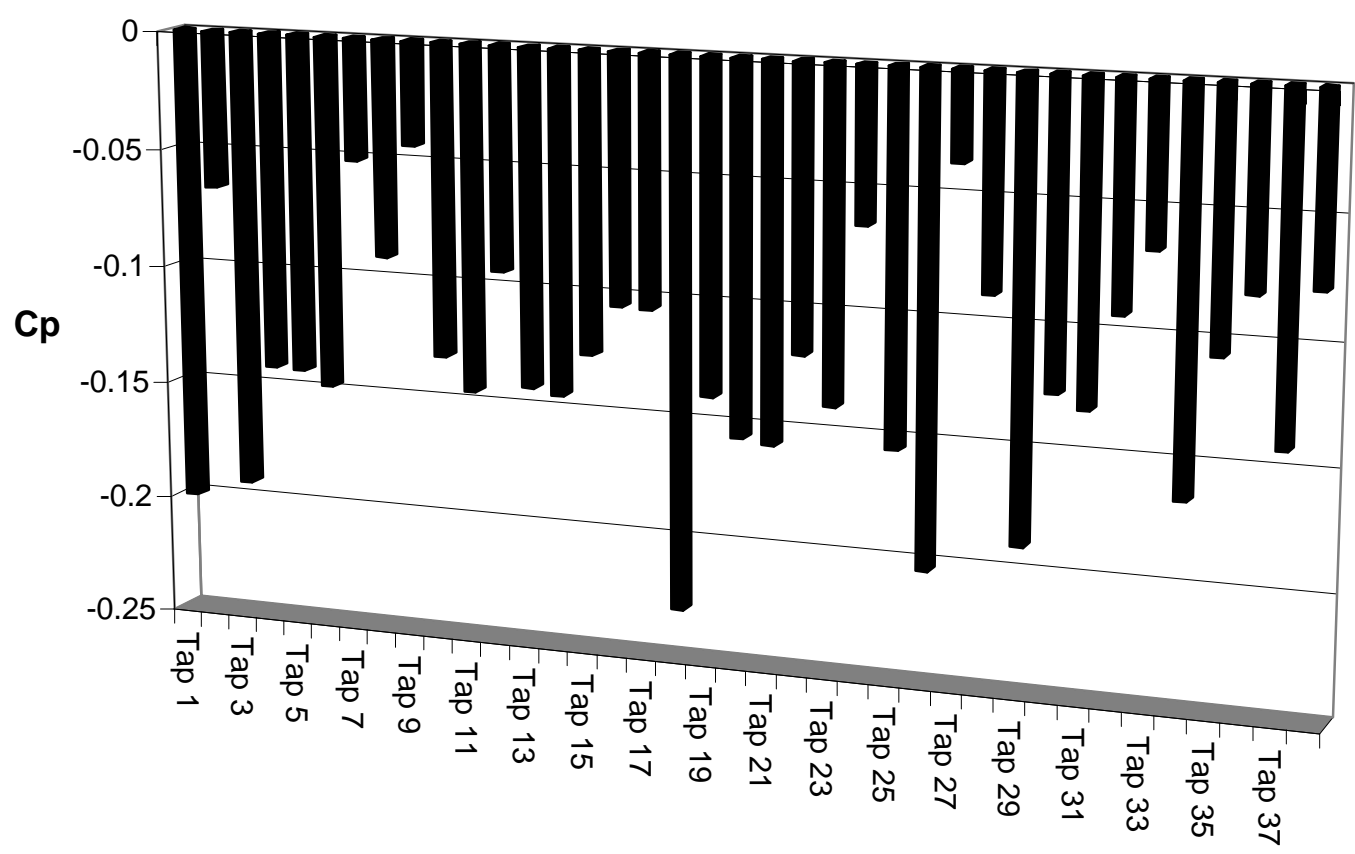

Figure A.19: Phase I Average Pressure Coefficients for 1/8-inch VG Modified Configuration 4 Test.

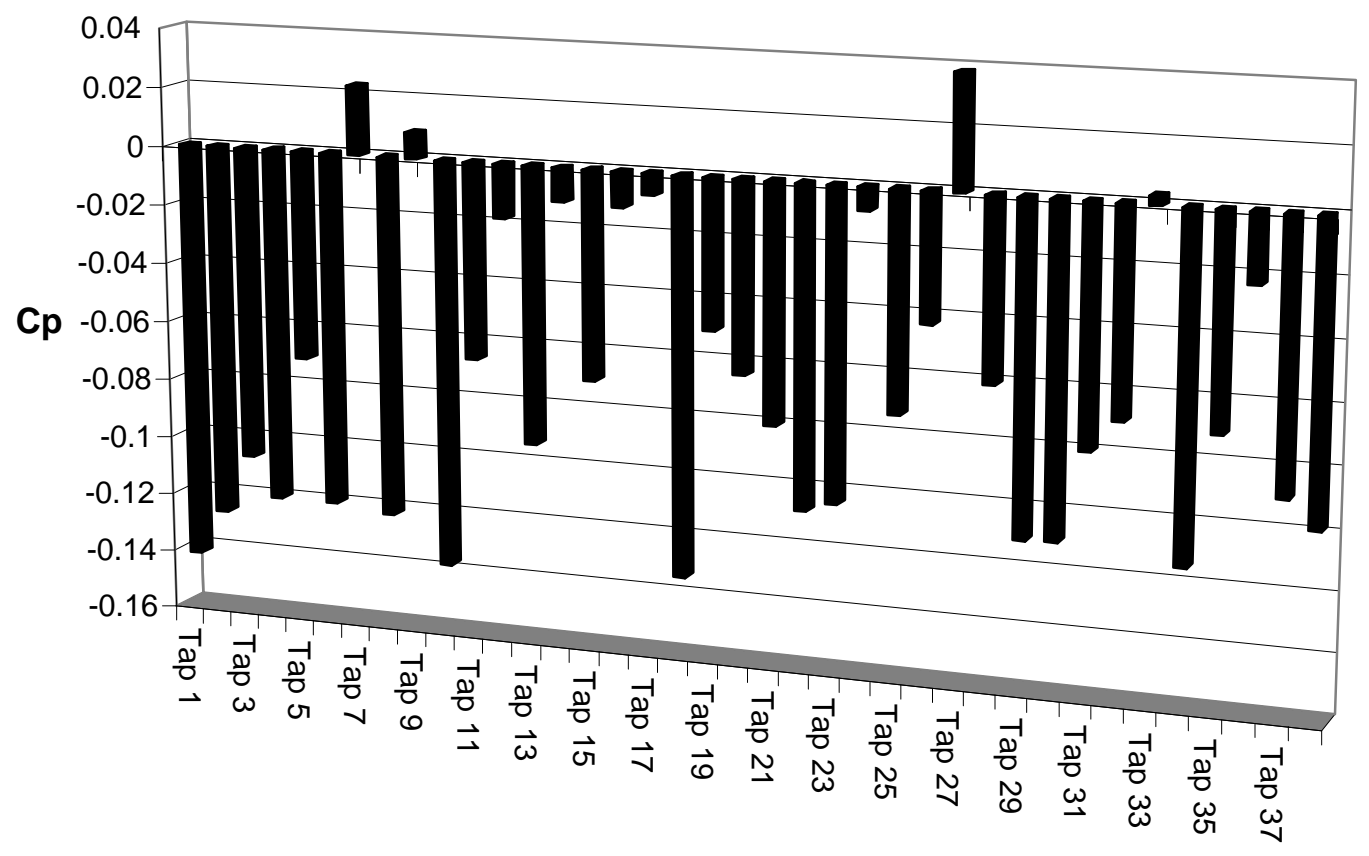

Figure A.20: Phase I Average Pressure Coefficients for 1/8-inch VG Modified Configuration 5 Test. 


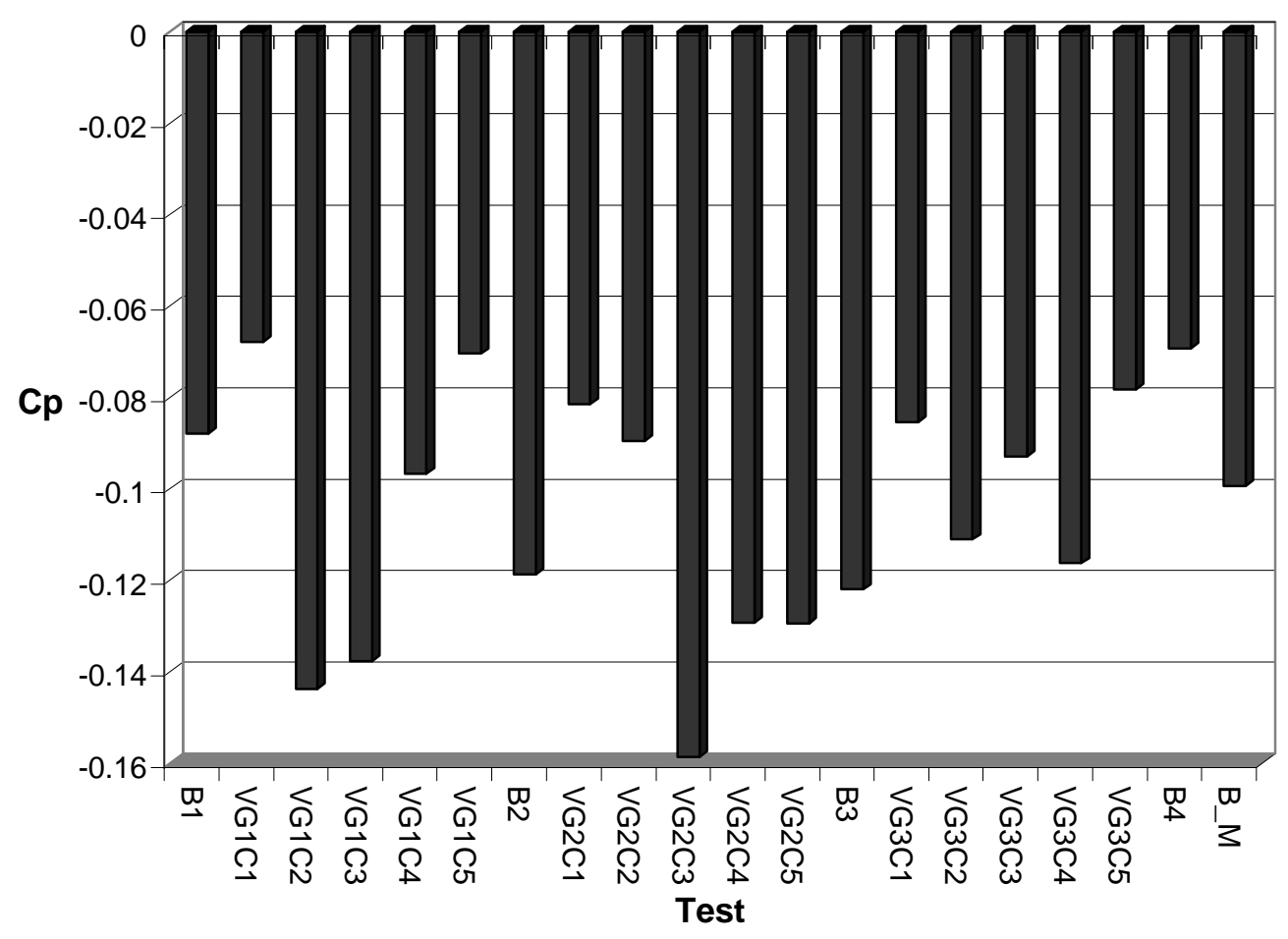

Figure A.21: Comparison of Phase I Average Pressure Coefficients for Pressure Tap \#5.

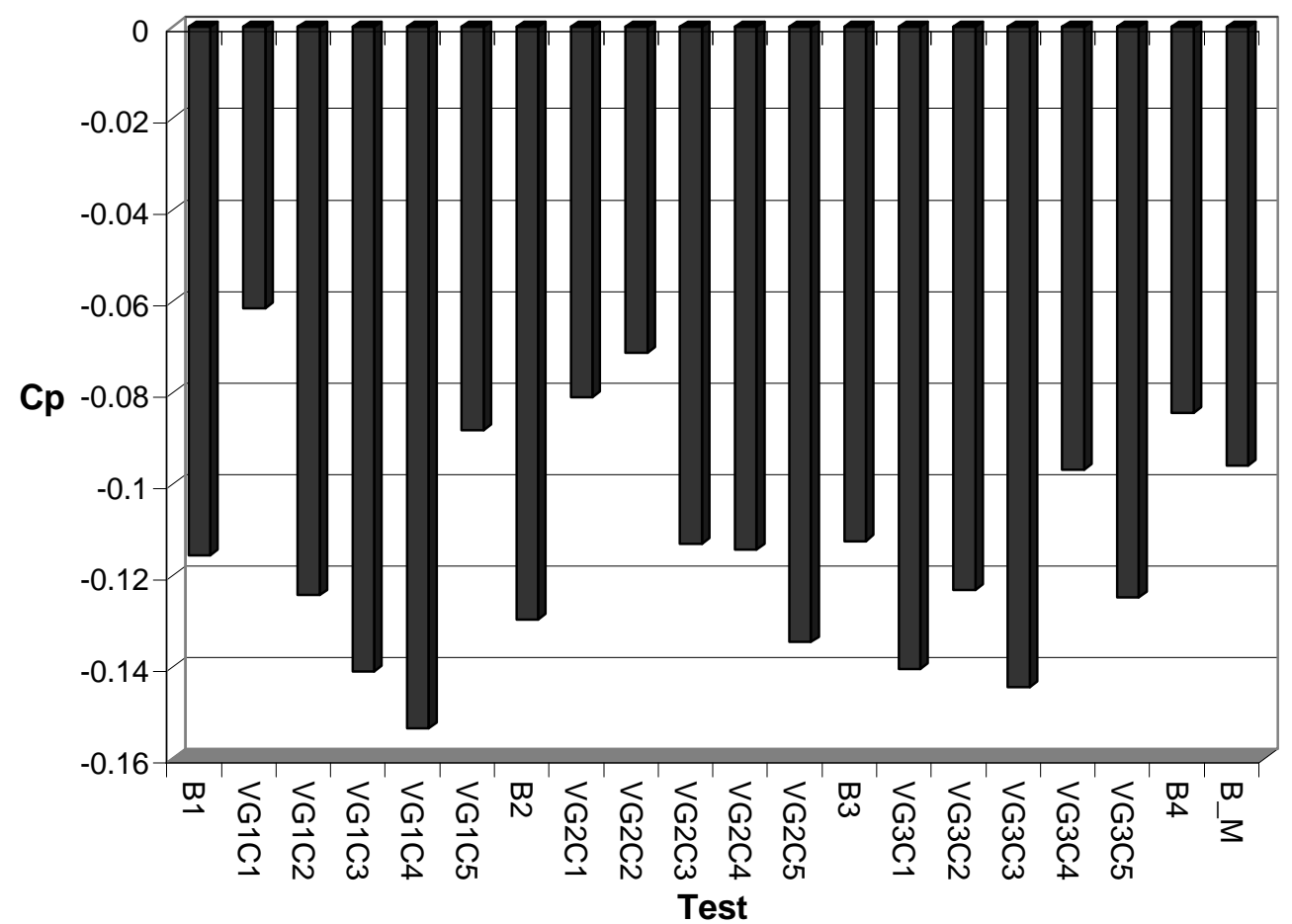

Figure A.22: Comparison of Phase I Average Pressure Coefficients for Pressure Tap \#6. 


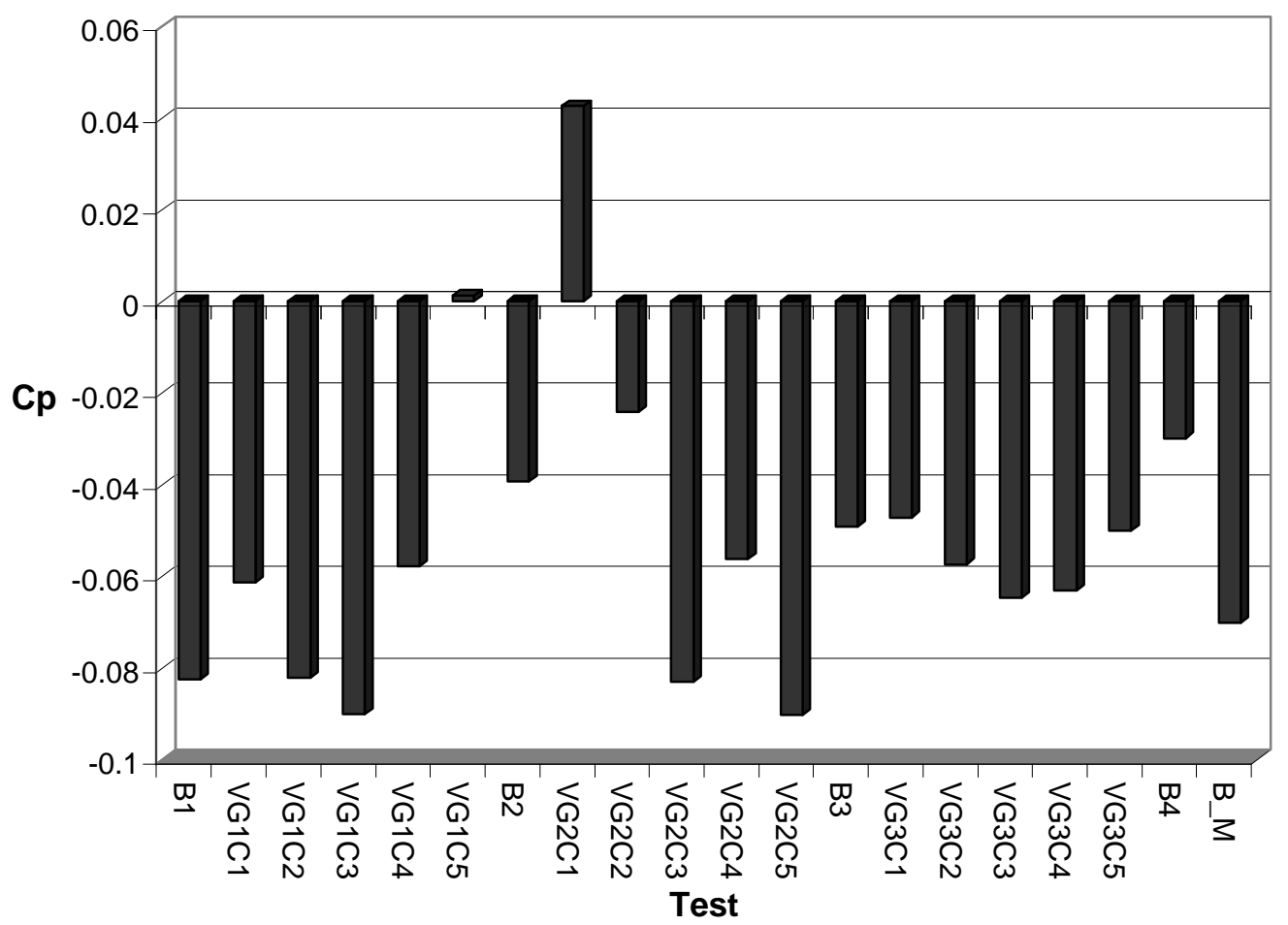

Figure A.23: Comparison of Phase I Average Pressure Coefficients for Pressure Tap \#24.

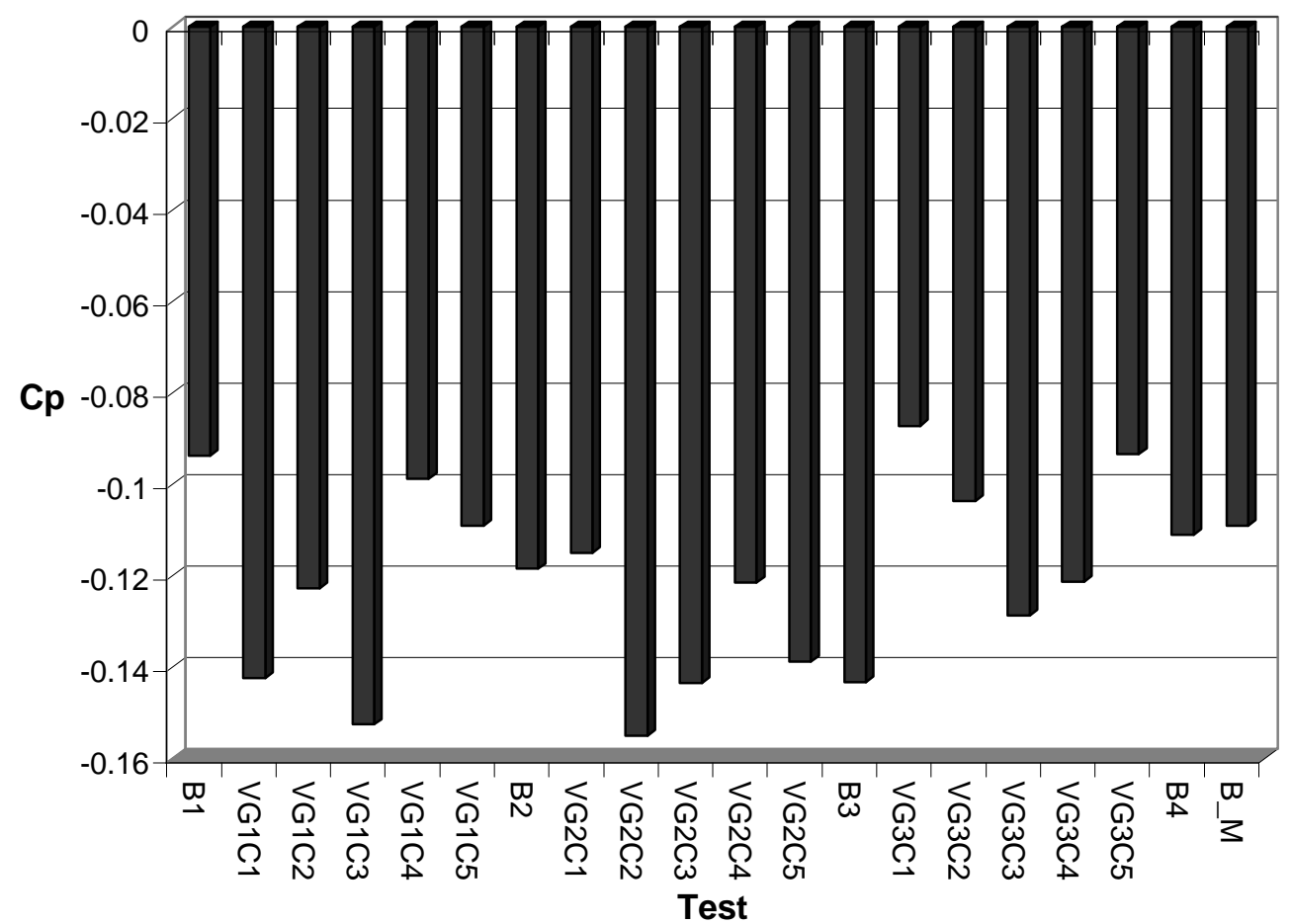

Figure A.24: Comparison of Phase I Average Pressure Coefficients for Pressure Tap \#25. 


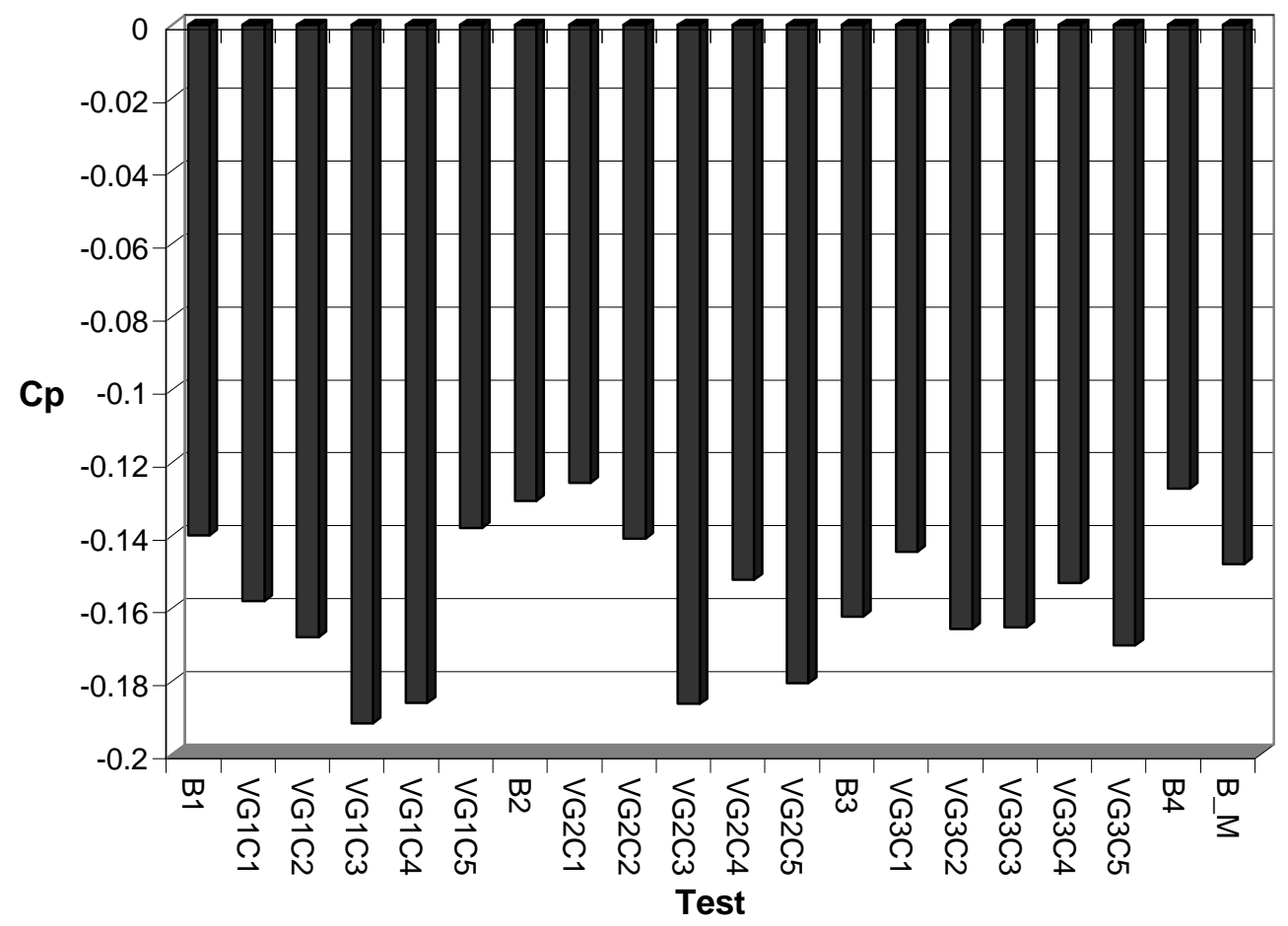

Figure A.25: Comparison of Phase I Average Pressure Coefficients for Pressure Tap \#26.

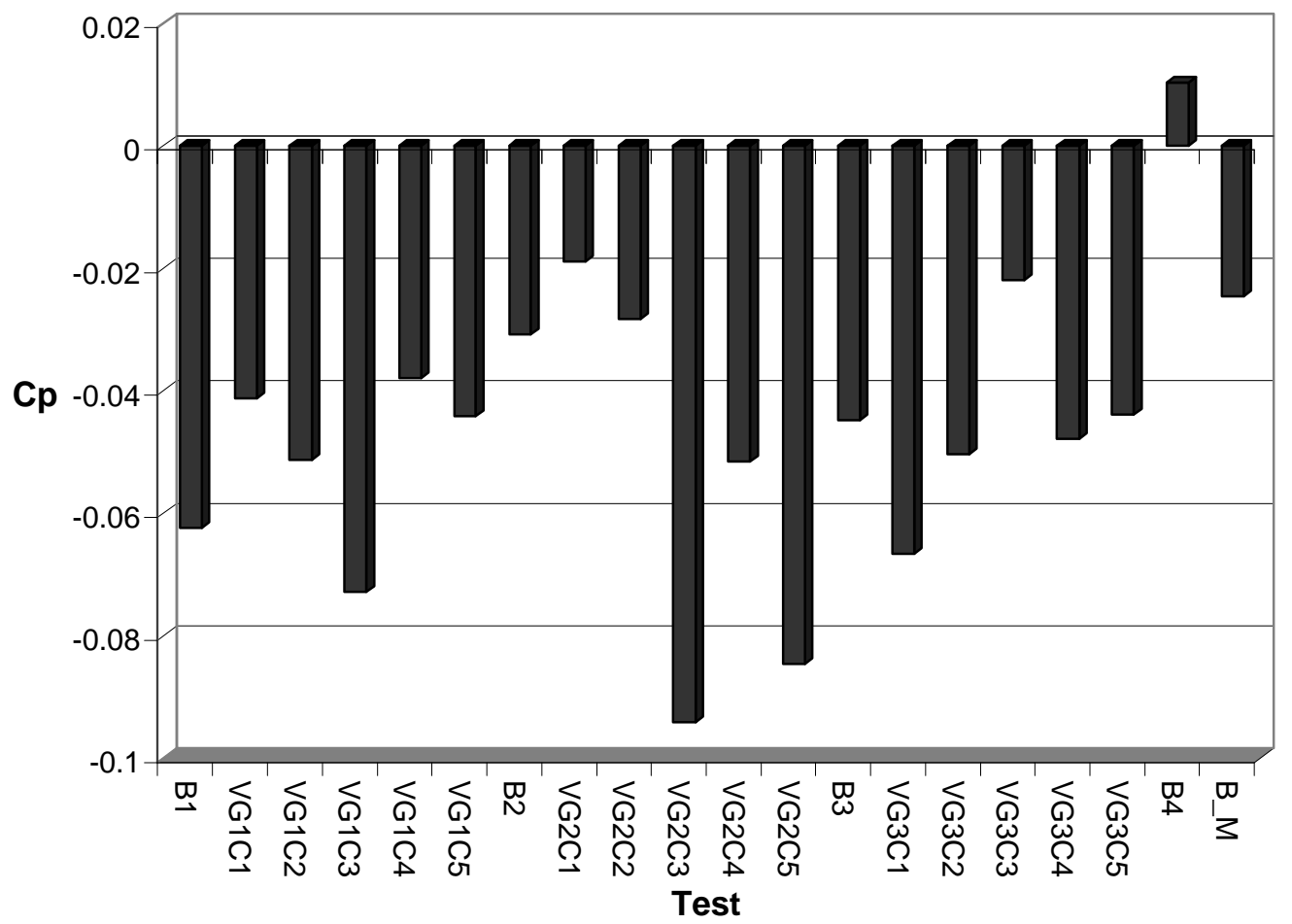

Figure A.26: Comparison of Phase I Average Pressure Coefficients for Pressure Tap \#27. 


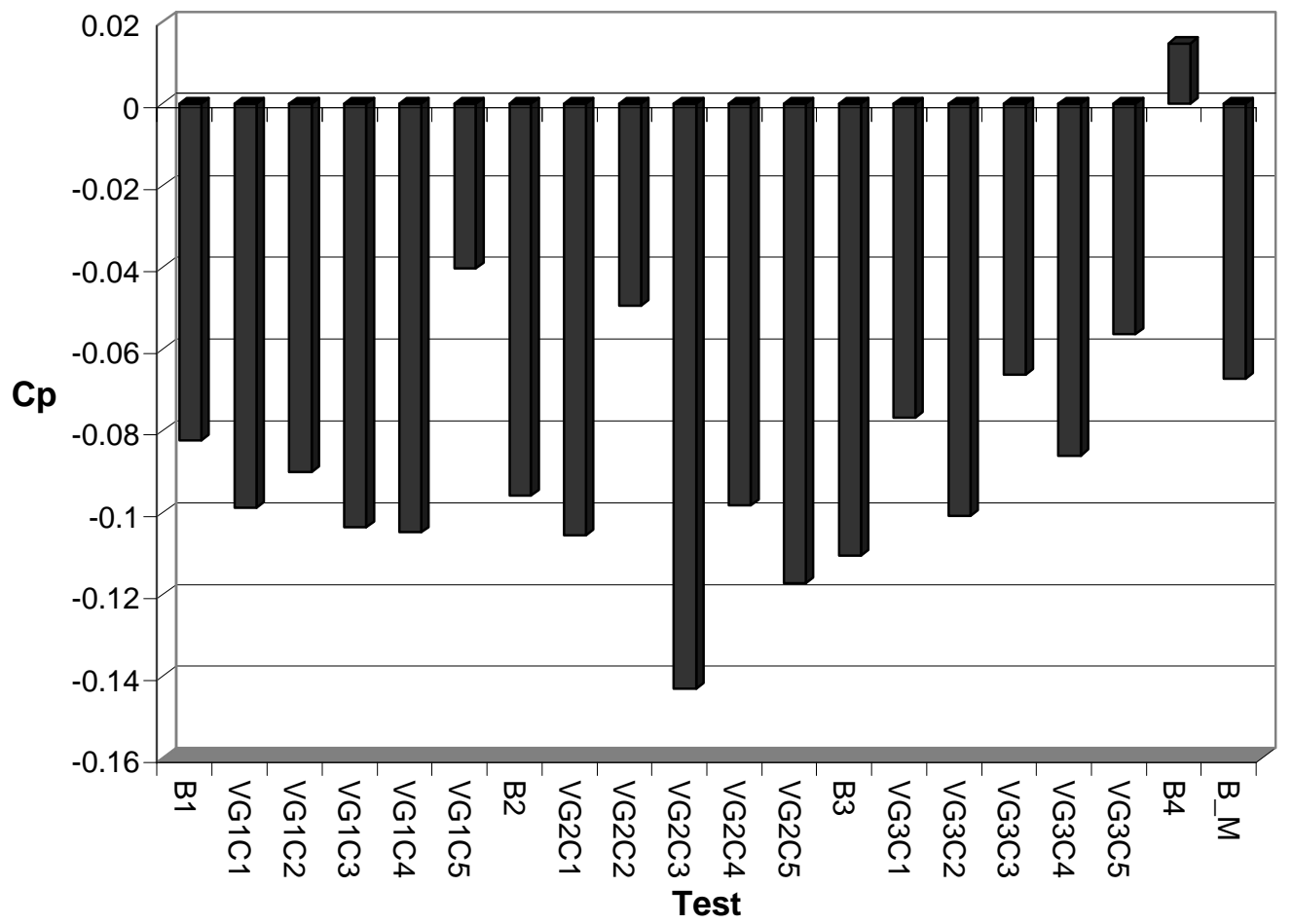

Figure A.27: Comparison of Phase I Average Pressure Coefficients for Pressure Tap \#28.

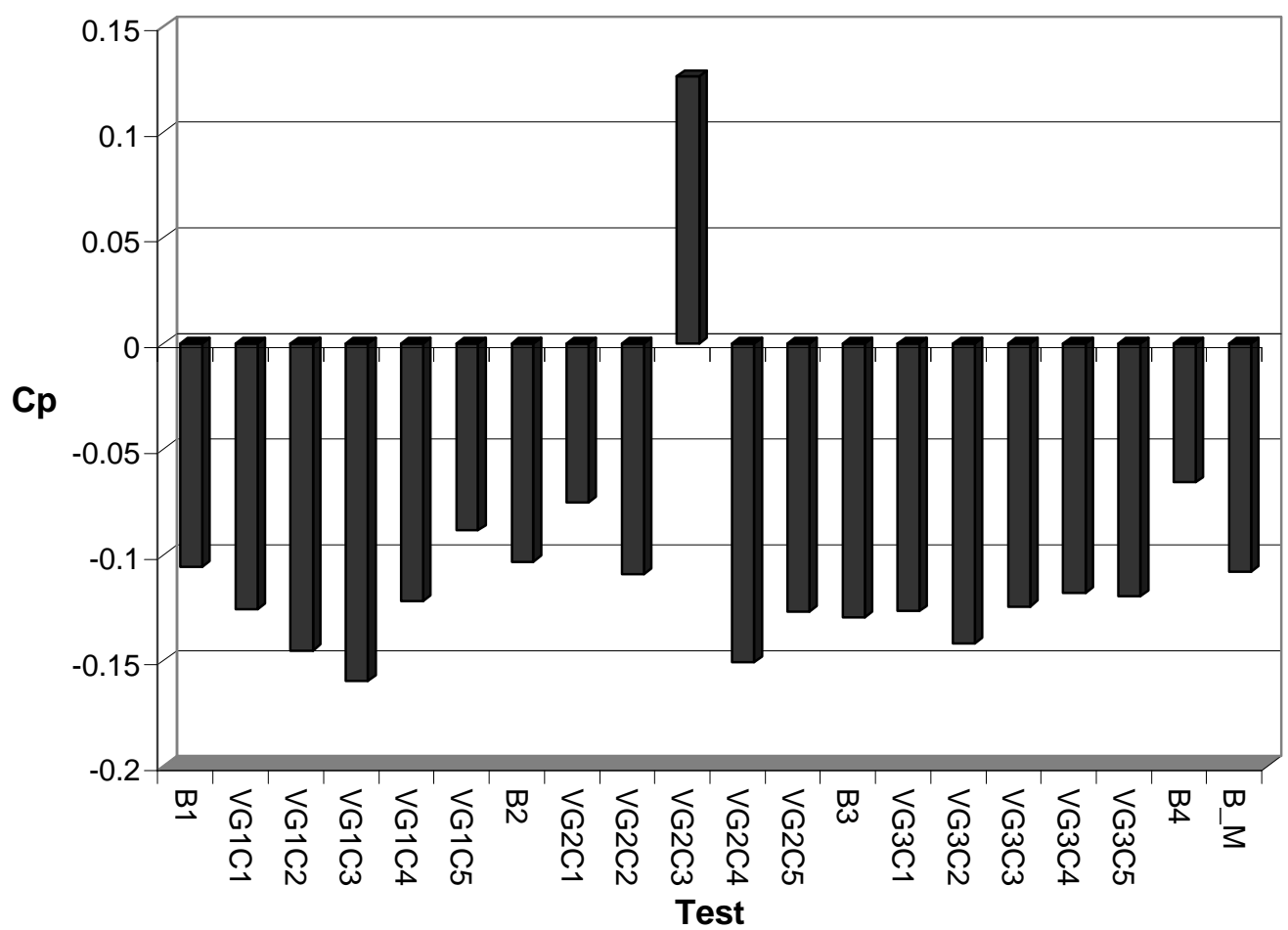

Figure A.28: Comparison of Phase I Average Pressure Coefficients for Pressure Tap \#29. 


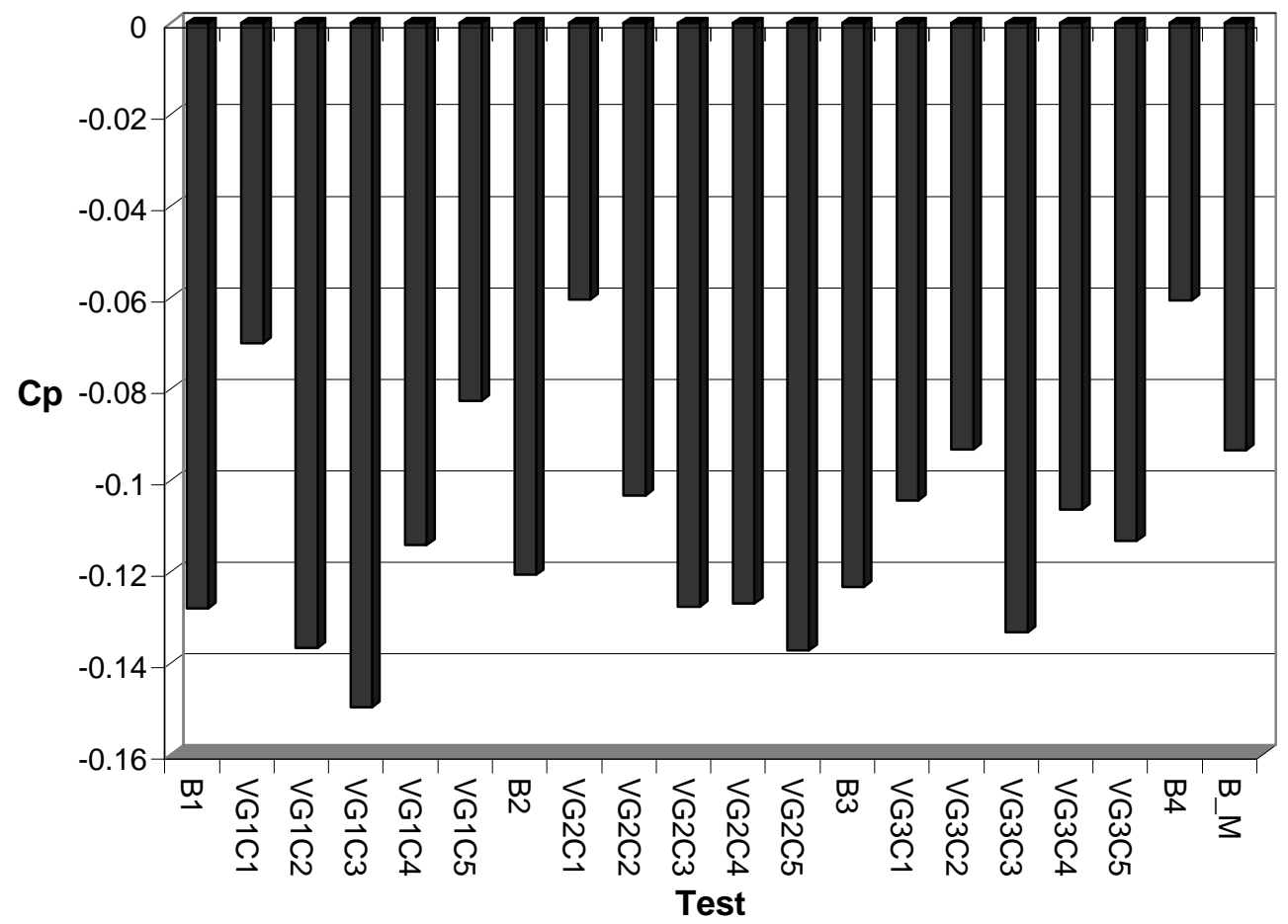

Figure A.29: Comparison of Phase I Average Pressure Coefficients for Pressure Tap \#30.

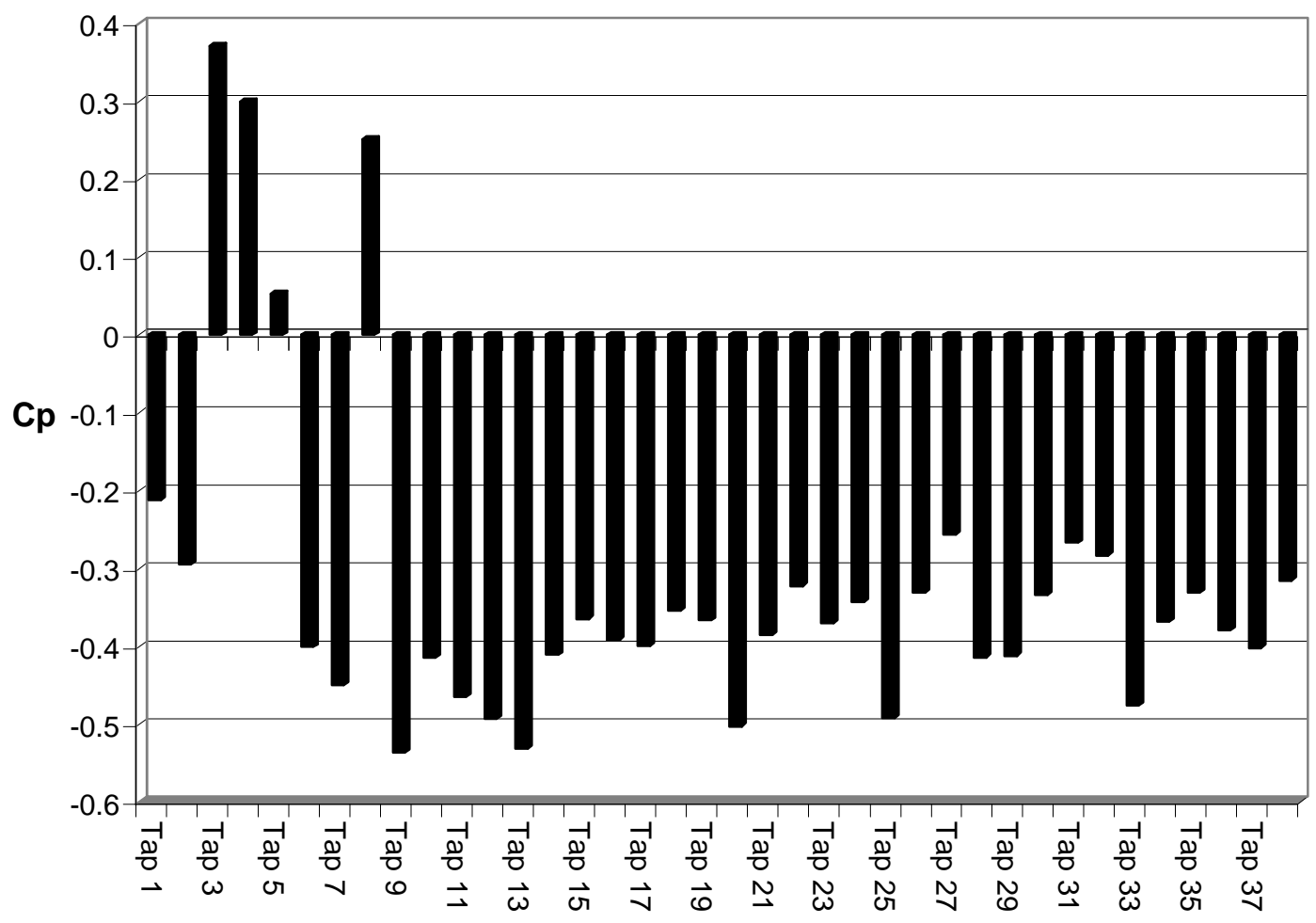

Figure A.30: Average Pressure Coefficients for Phase II Run 4.01. 


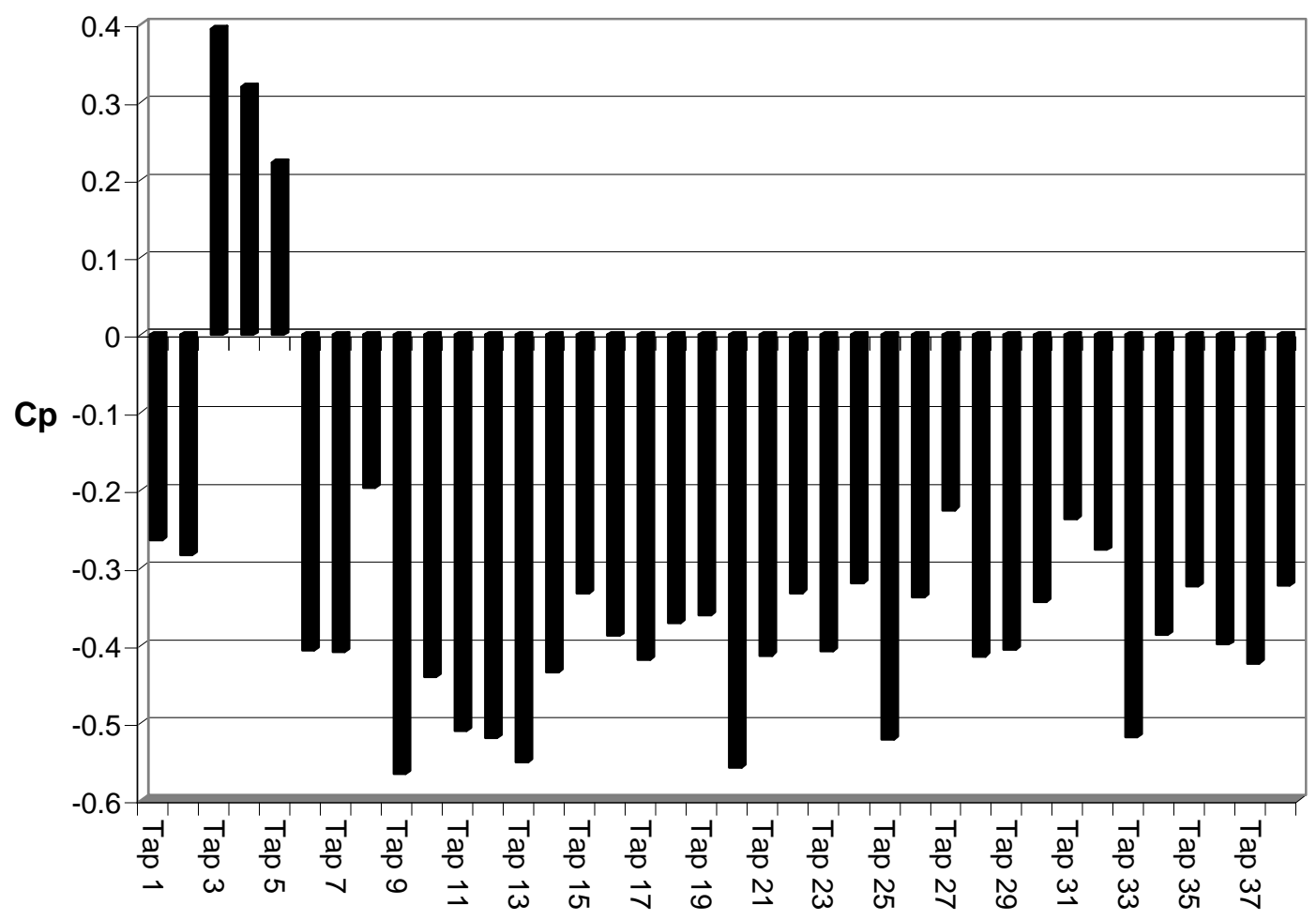

Figure A.31: Average Pressure Coefficients for Phase II Run 4.03.

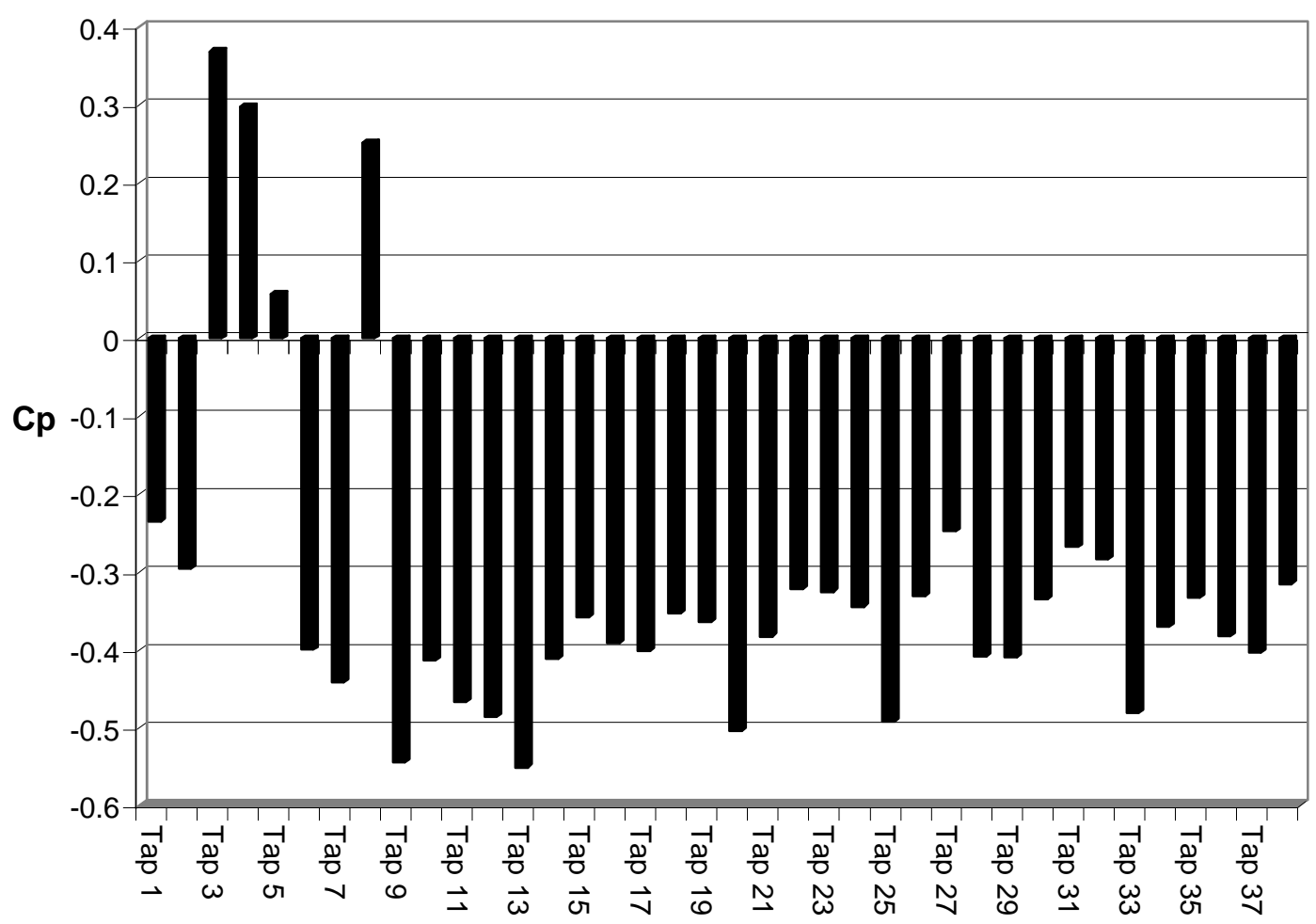

Figure A.32: Average Pressure Coefficients for Phase II Run 5.01. 


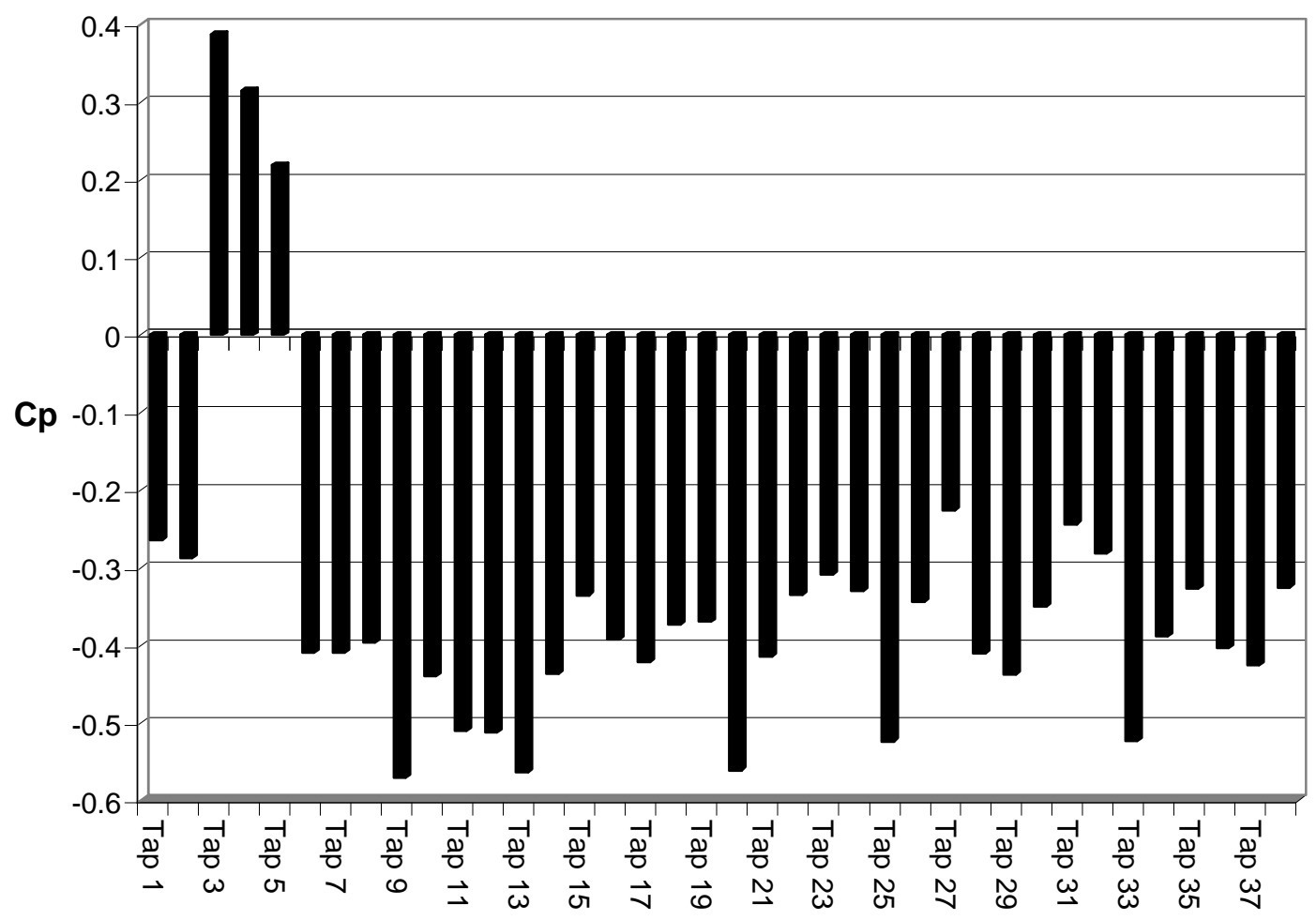

Figure A.33: Average Pressure Coefficients for Phase II Run 5.03.

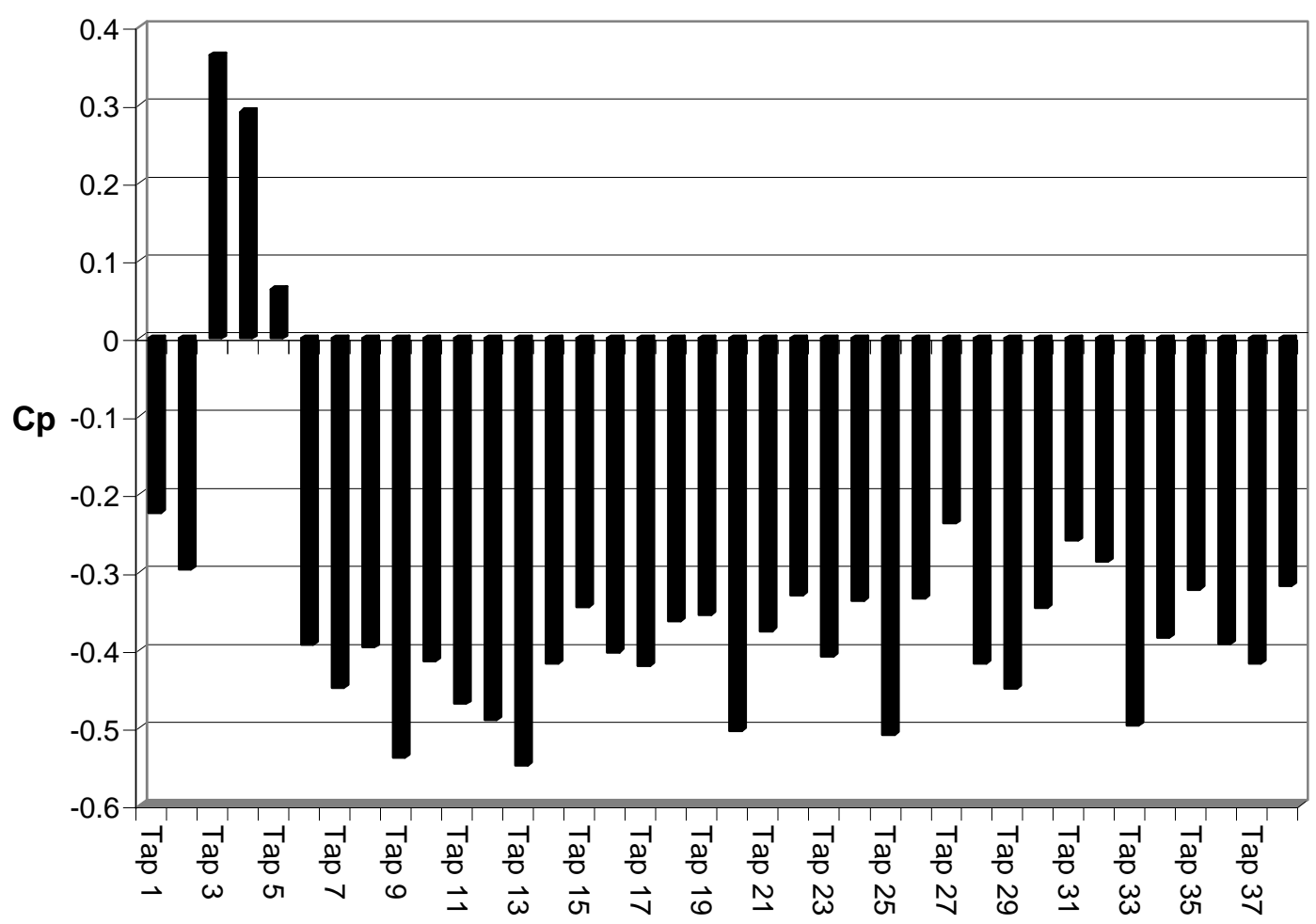

Figure A.34: Average Pressure Coefficients for Phase II Run 6.01. 


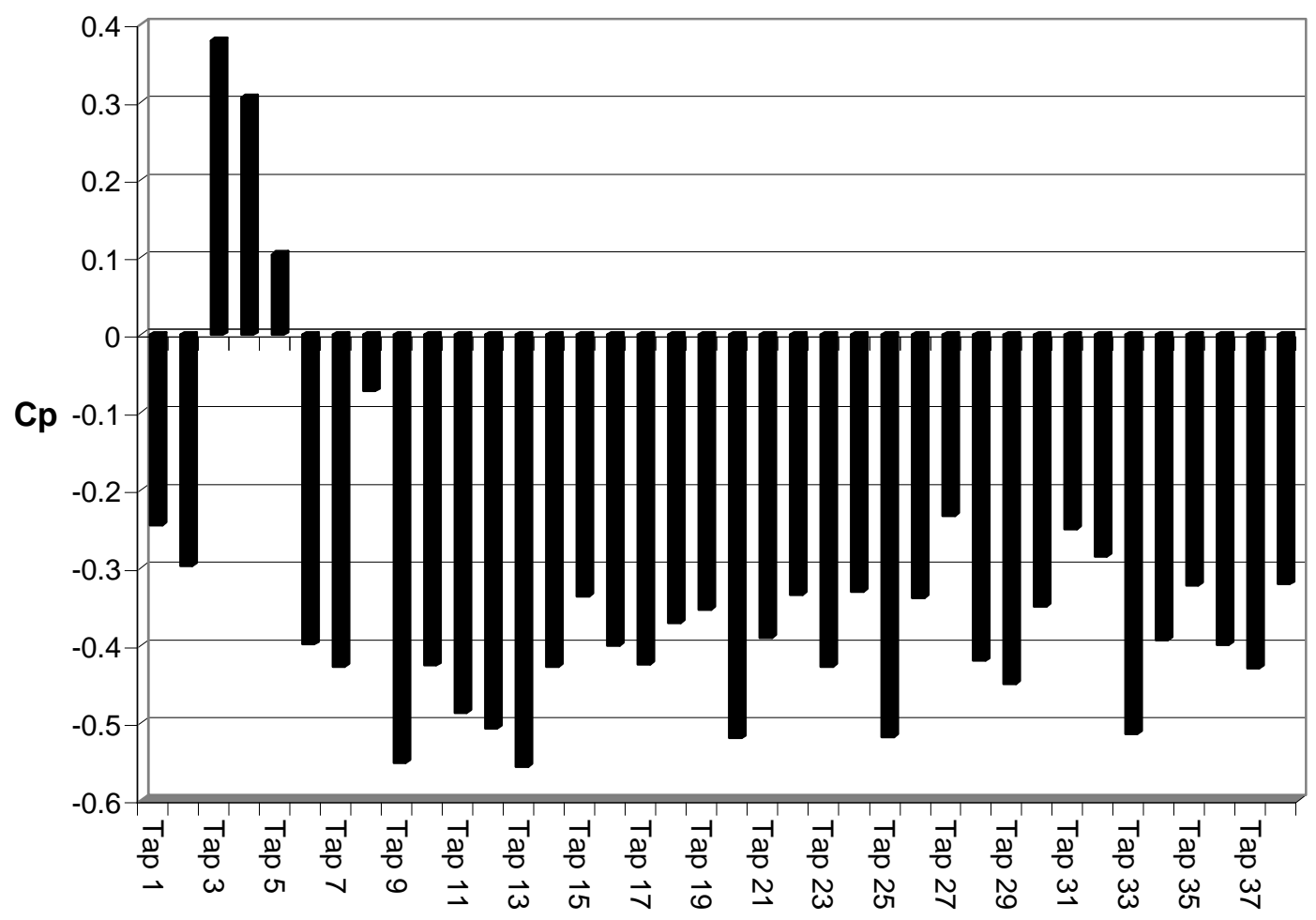

Figure A.35: Average Pressure Coefficients for Phase II Run 6.02.

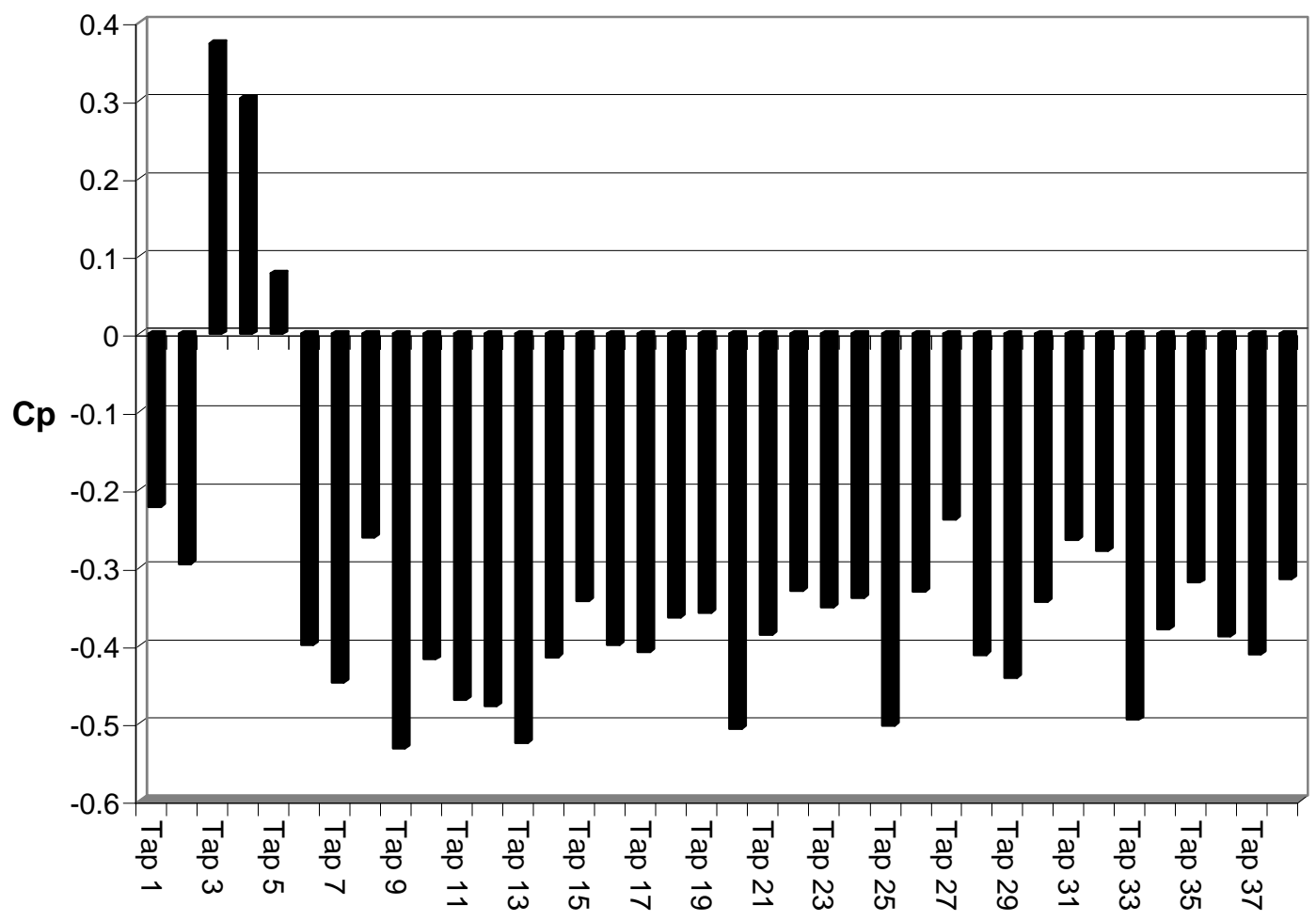

Figure A.36: Average Pressure Coefficients for Phase II Run 7.01. 


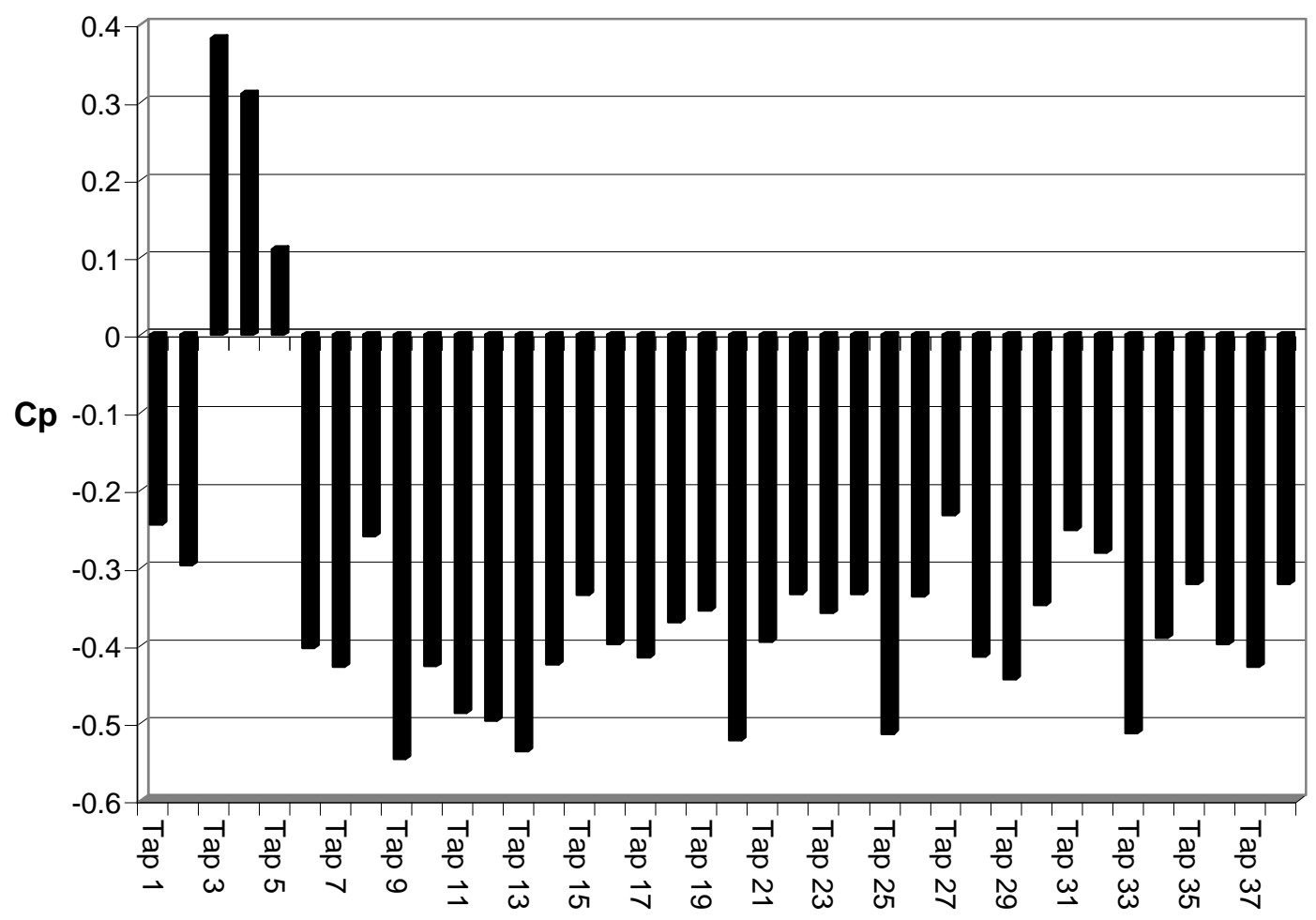

Figure A.37: Average Pressure Coefficients for Phase II Run 7.02.

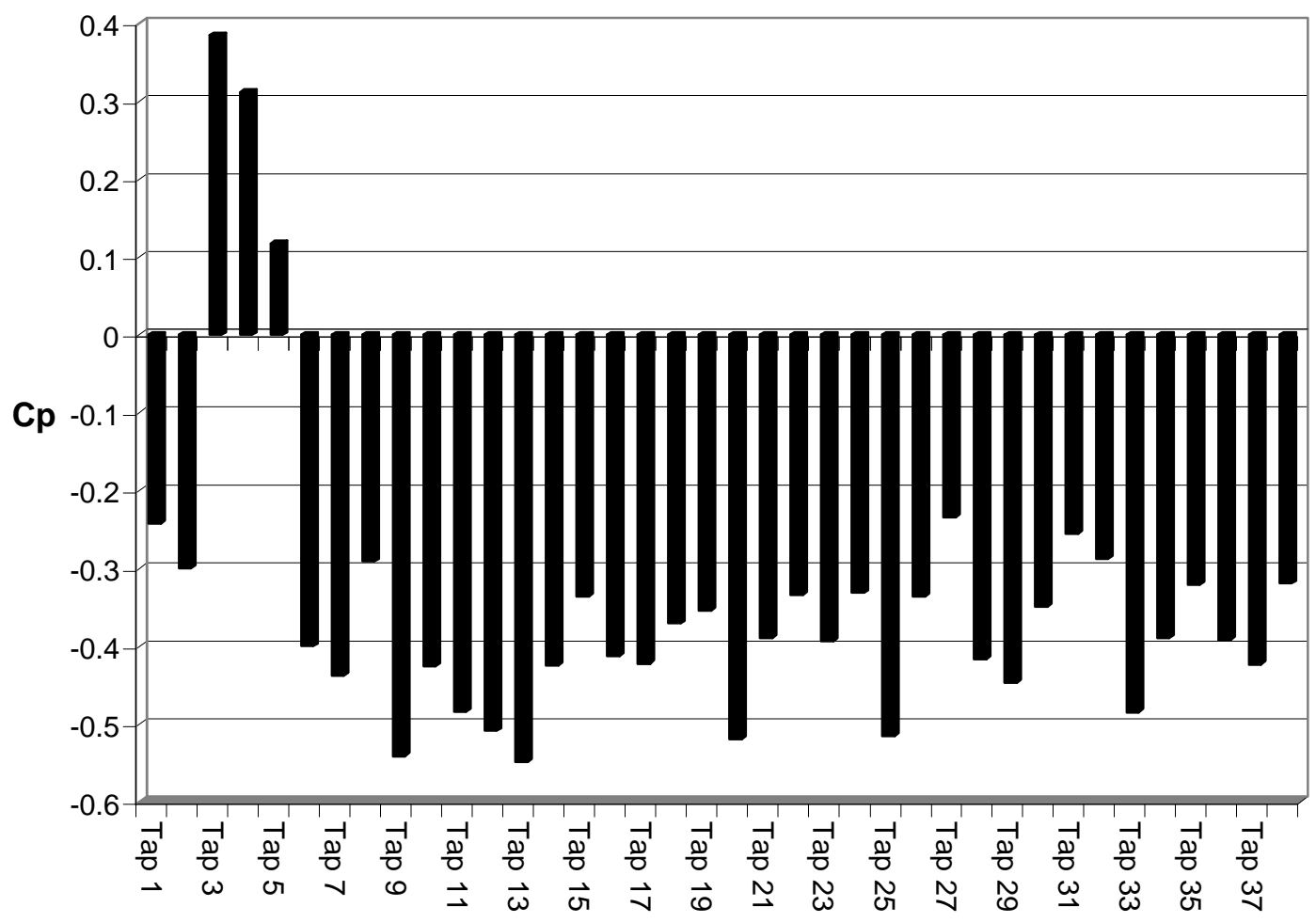

Figure A.38: Average Pressure Coefficients for Phase II Run 8.02. 


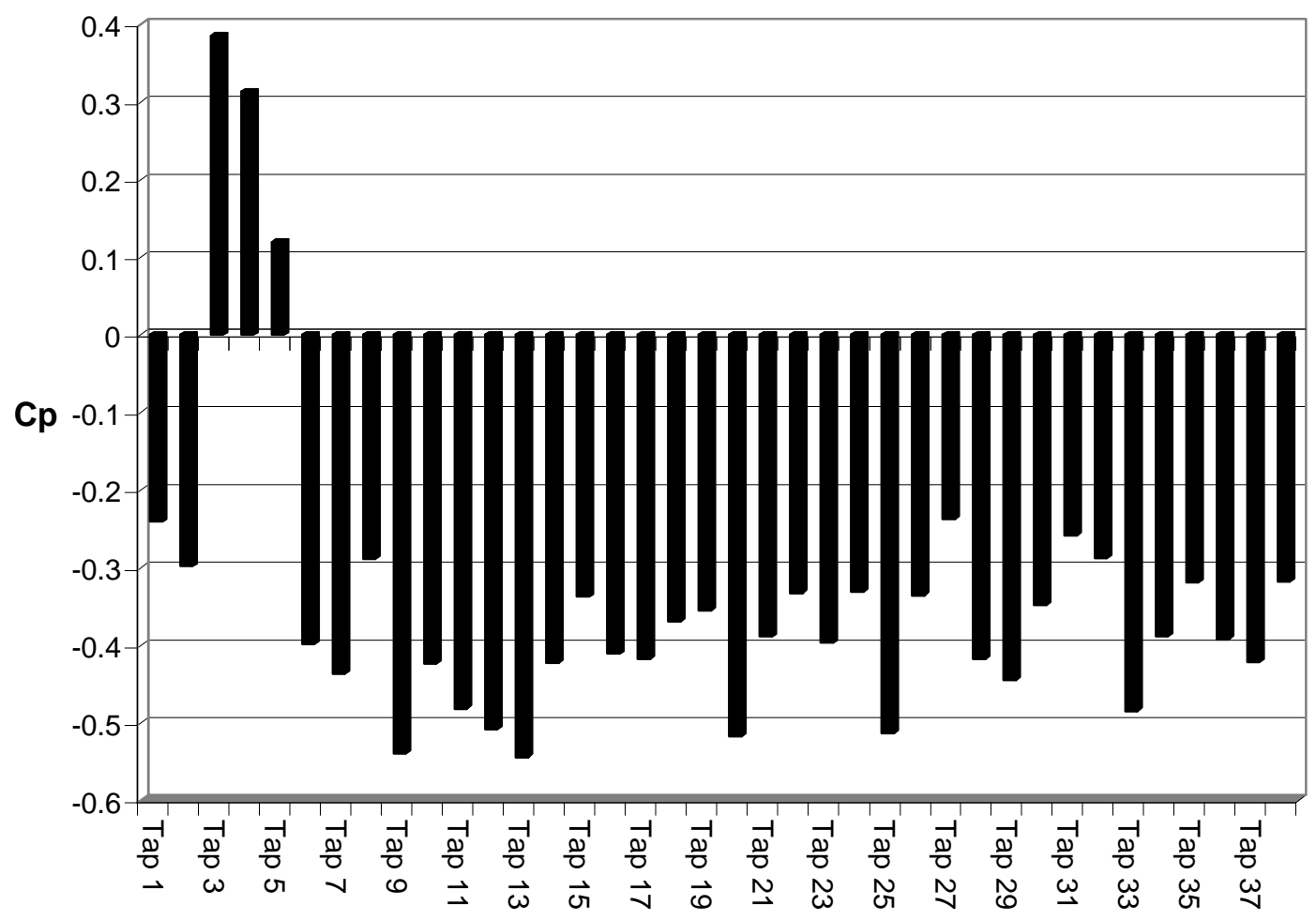

Figure A.39: Average Pressure Coefficients for Phase II Run 8.03.

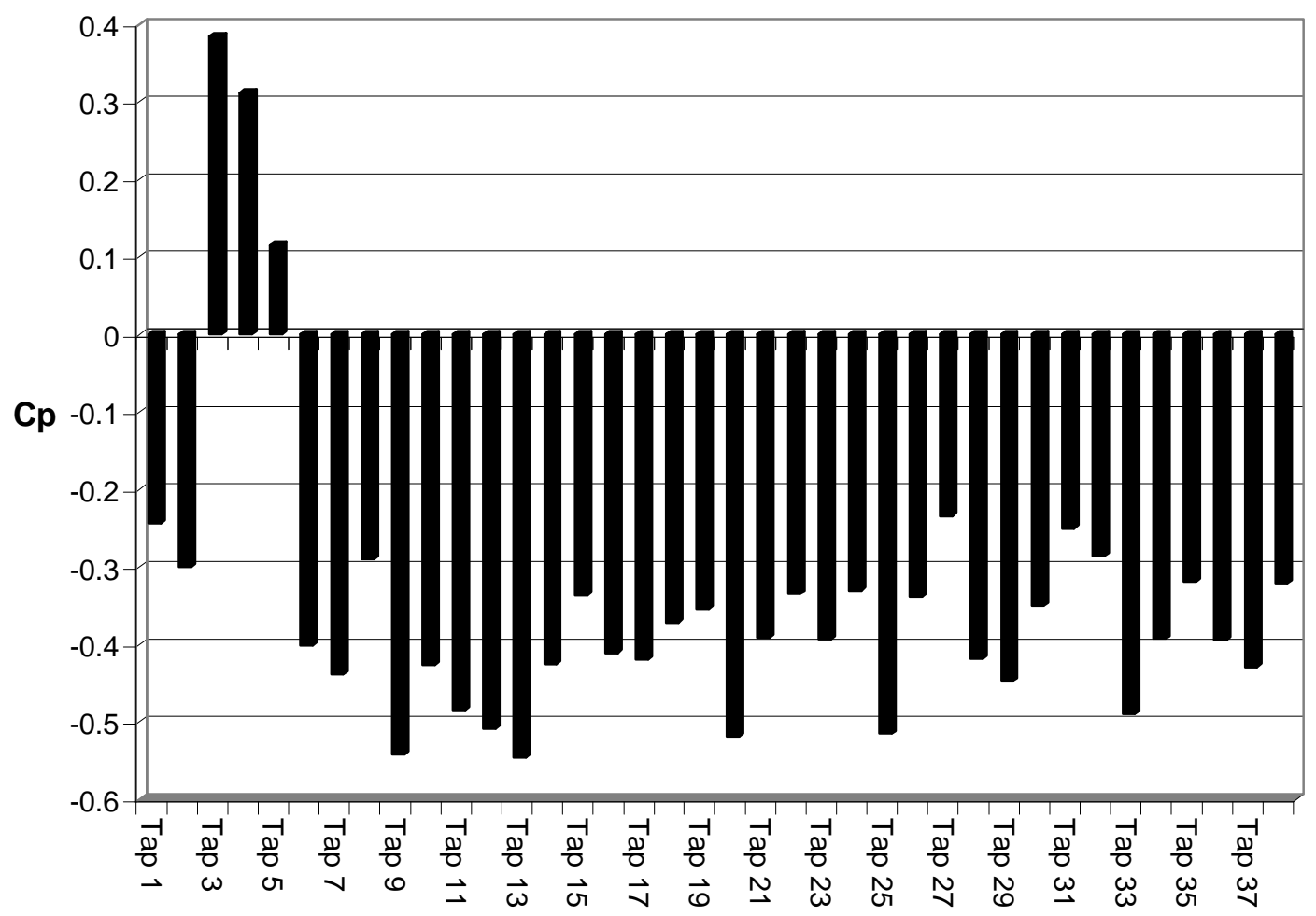

Figure A.40: Average Pressure Coefficients for Phase II Run 8.04. 


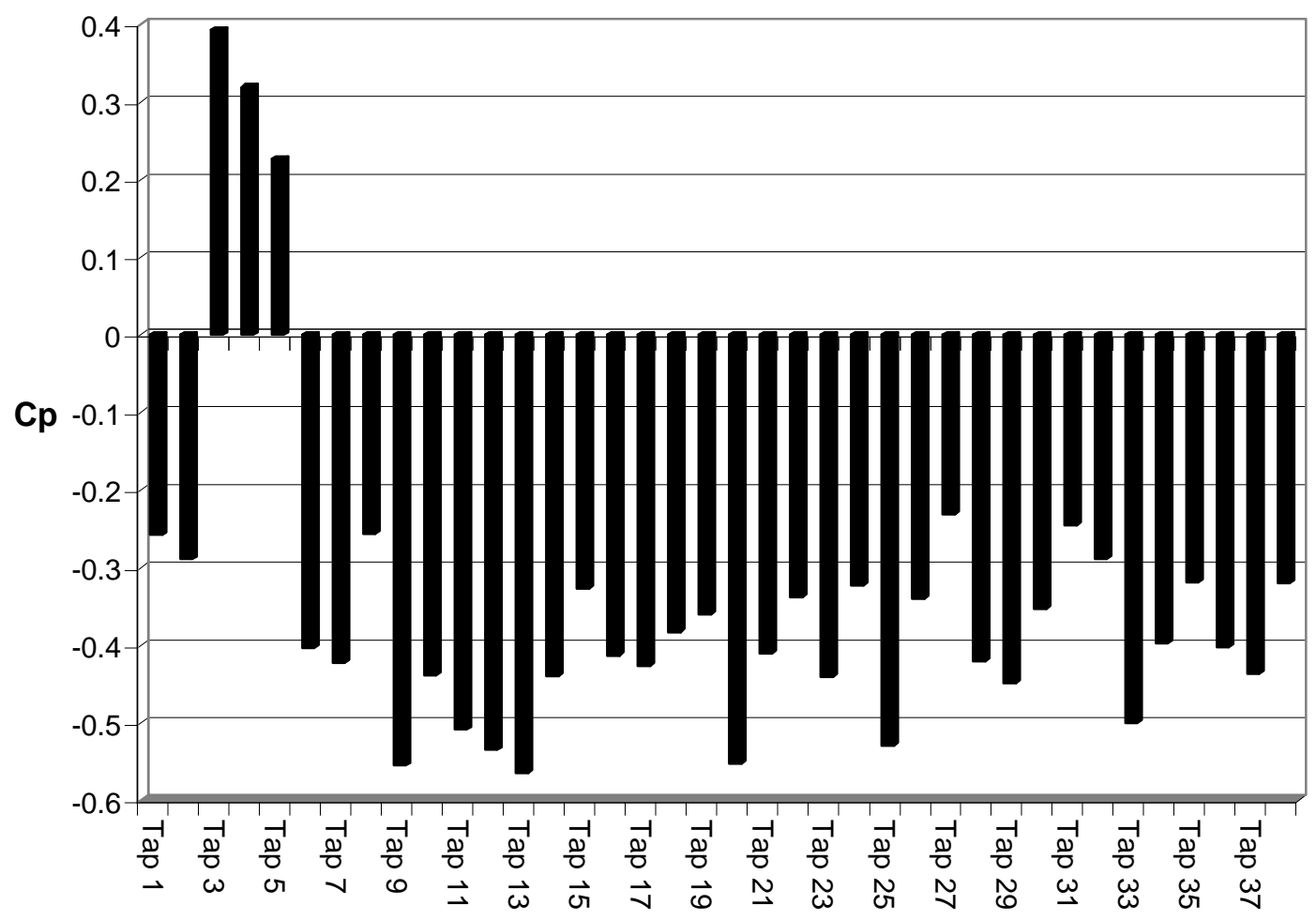

Figure A.41: Average Pressure Coefficients for Phase II Run 8.05.

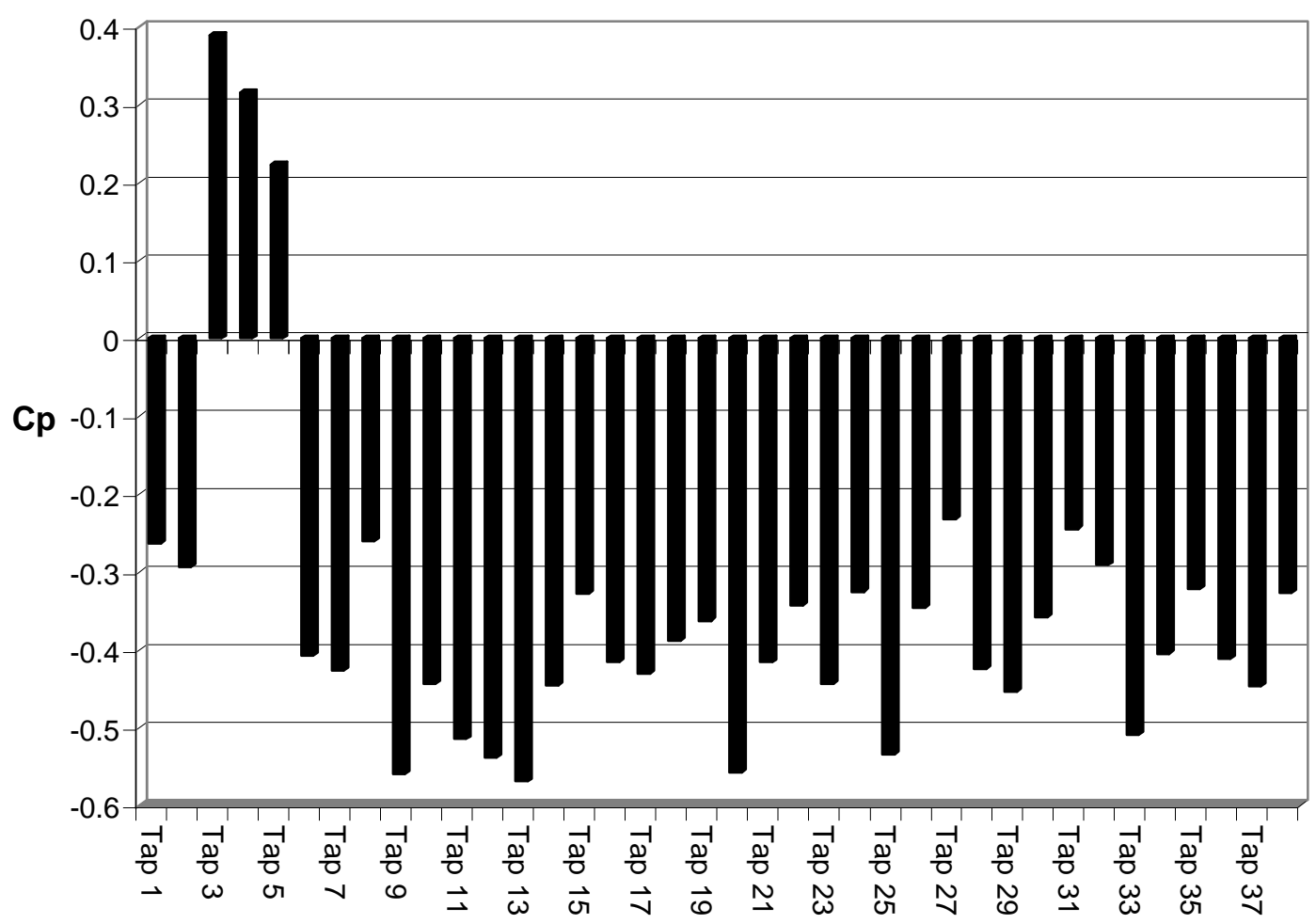

Figure A.42: Average Pressure Coefficients for Phase II Run 8.06. 


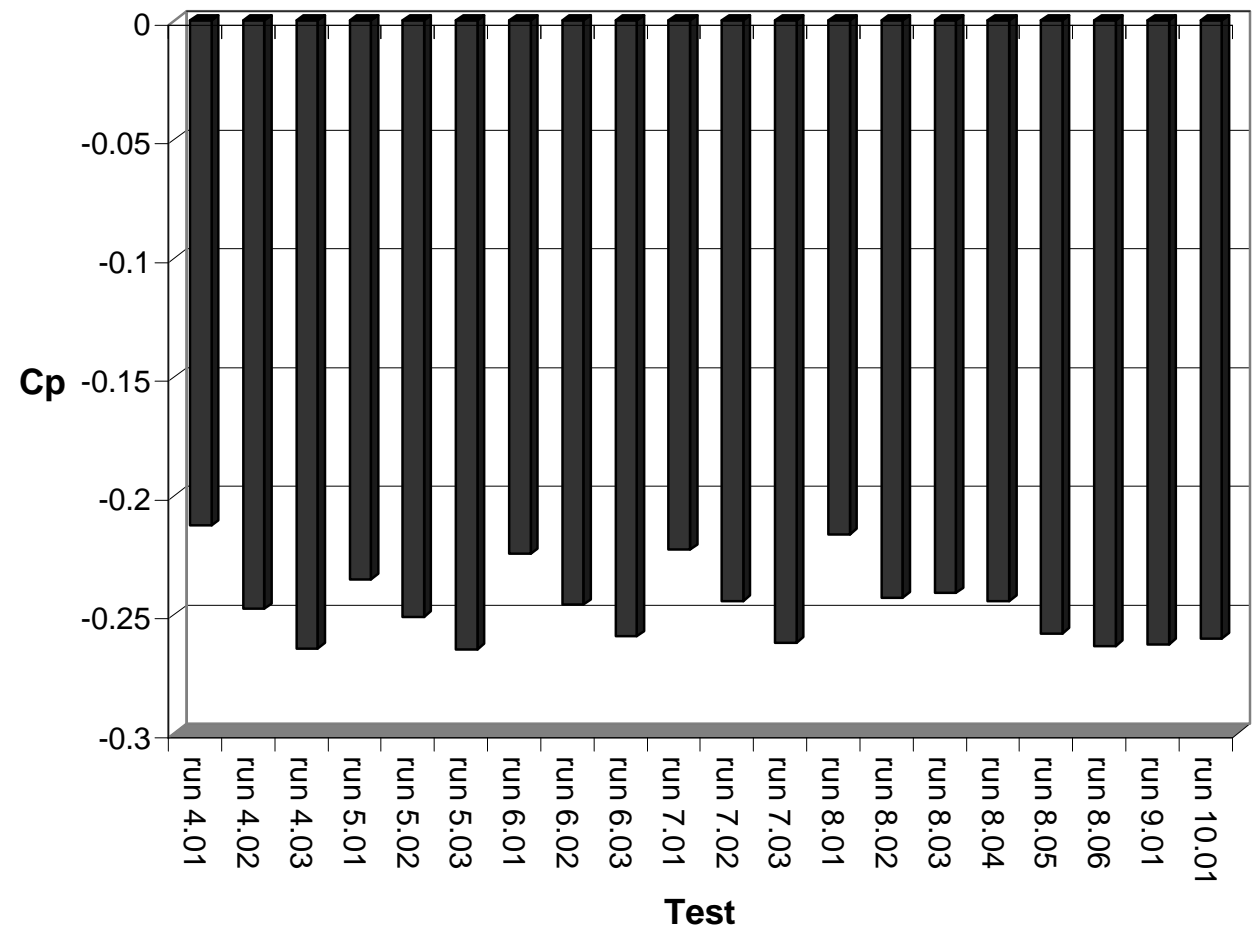

Figure A.43: Comparison of Phase II Average Pressure Coefficients for Pressure Tap \#1.

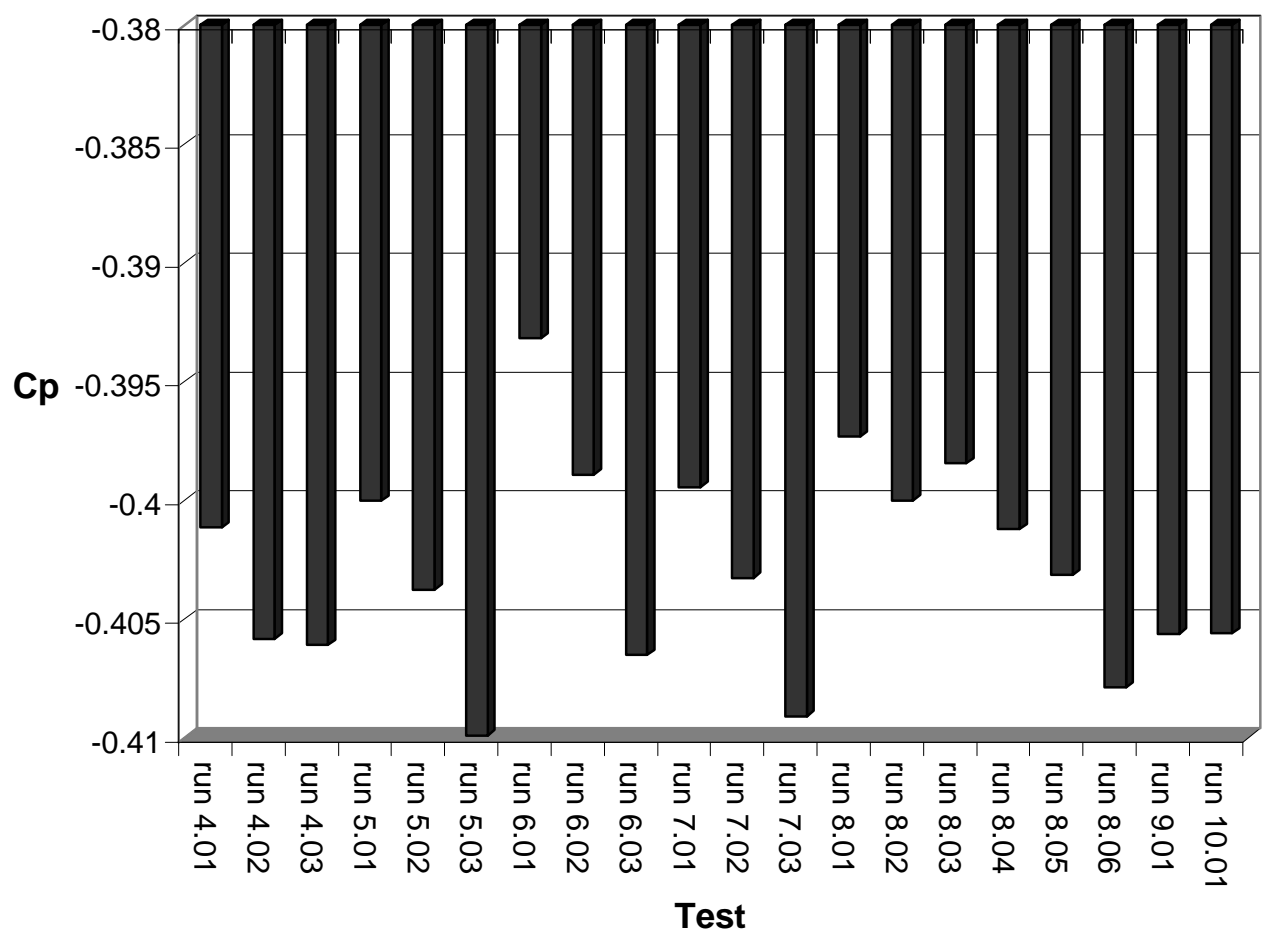

Figure A.44: Comparison of Phase II Average Pressure Coefficients for Pressure Tap \#6. 


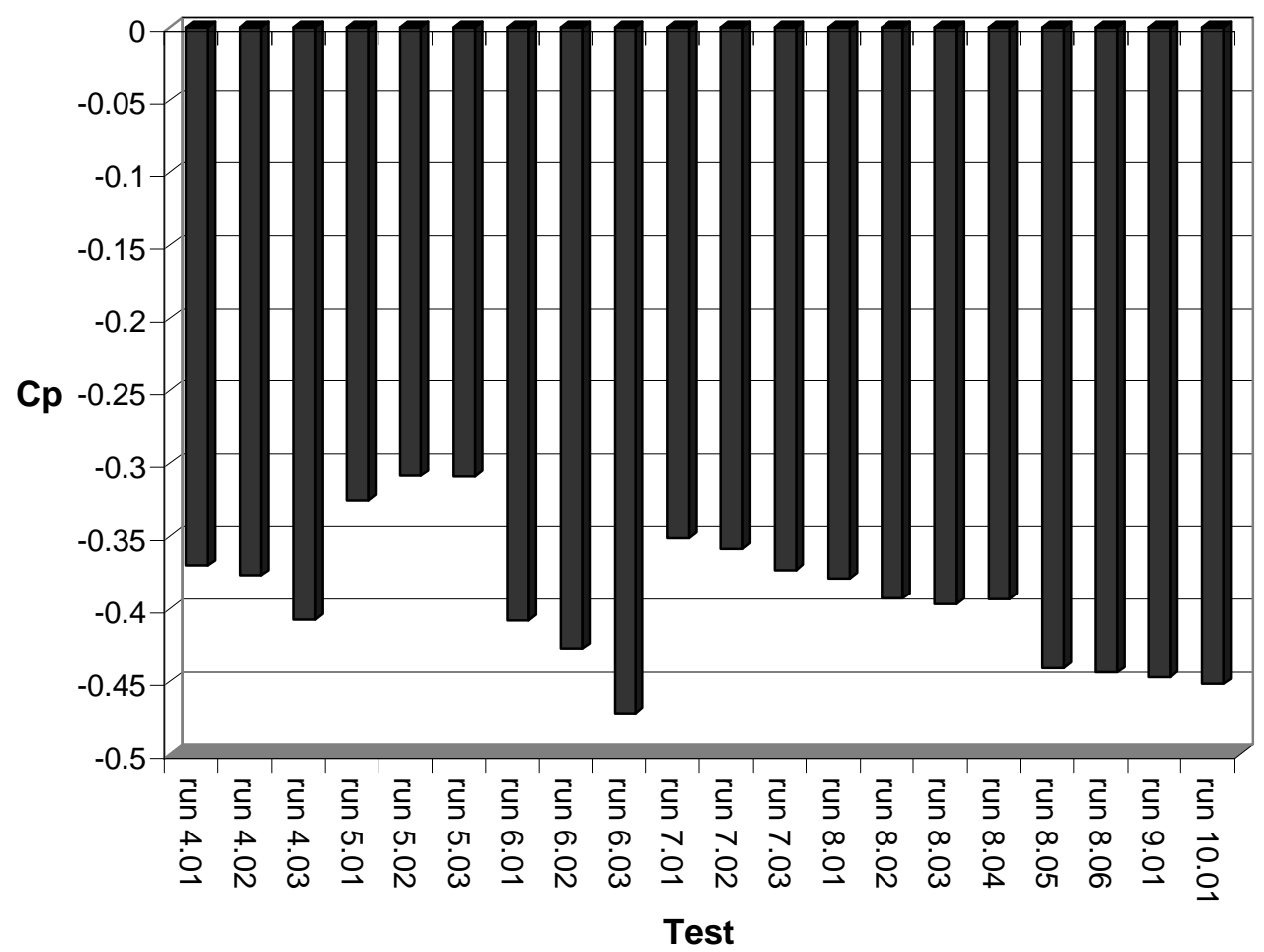

Figure A.45: Comparison of Phase II Average Pressure Coefficients for Pressure Tap \#23.

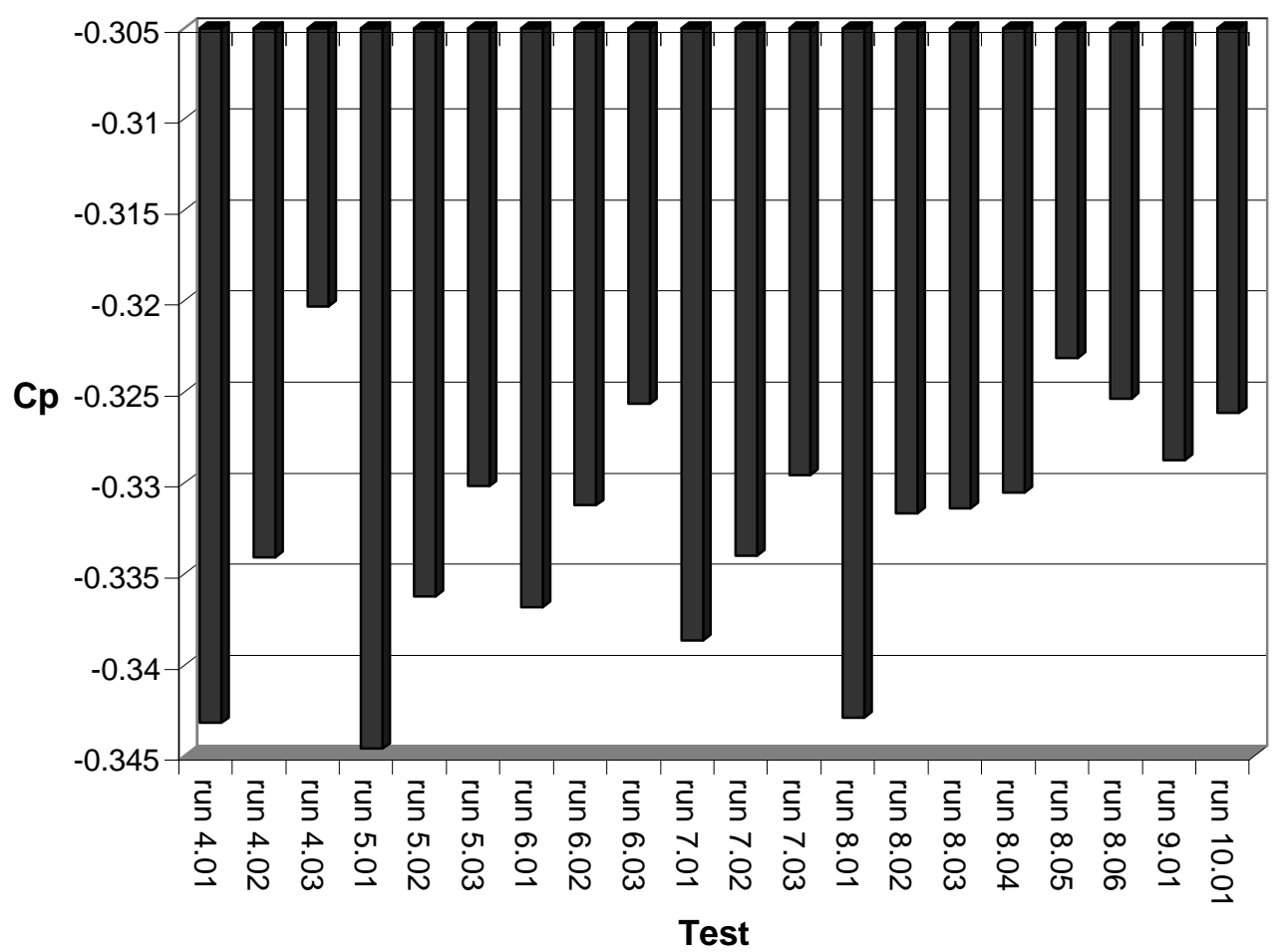

Figure A.46: Comparison of Phase II Average Pressure Coefficients for Pressure Tap \#24. 


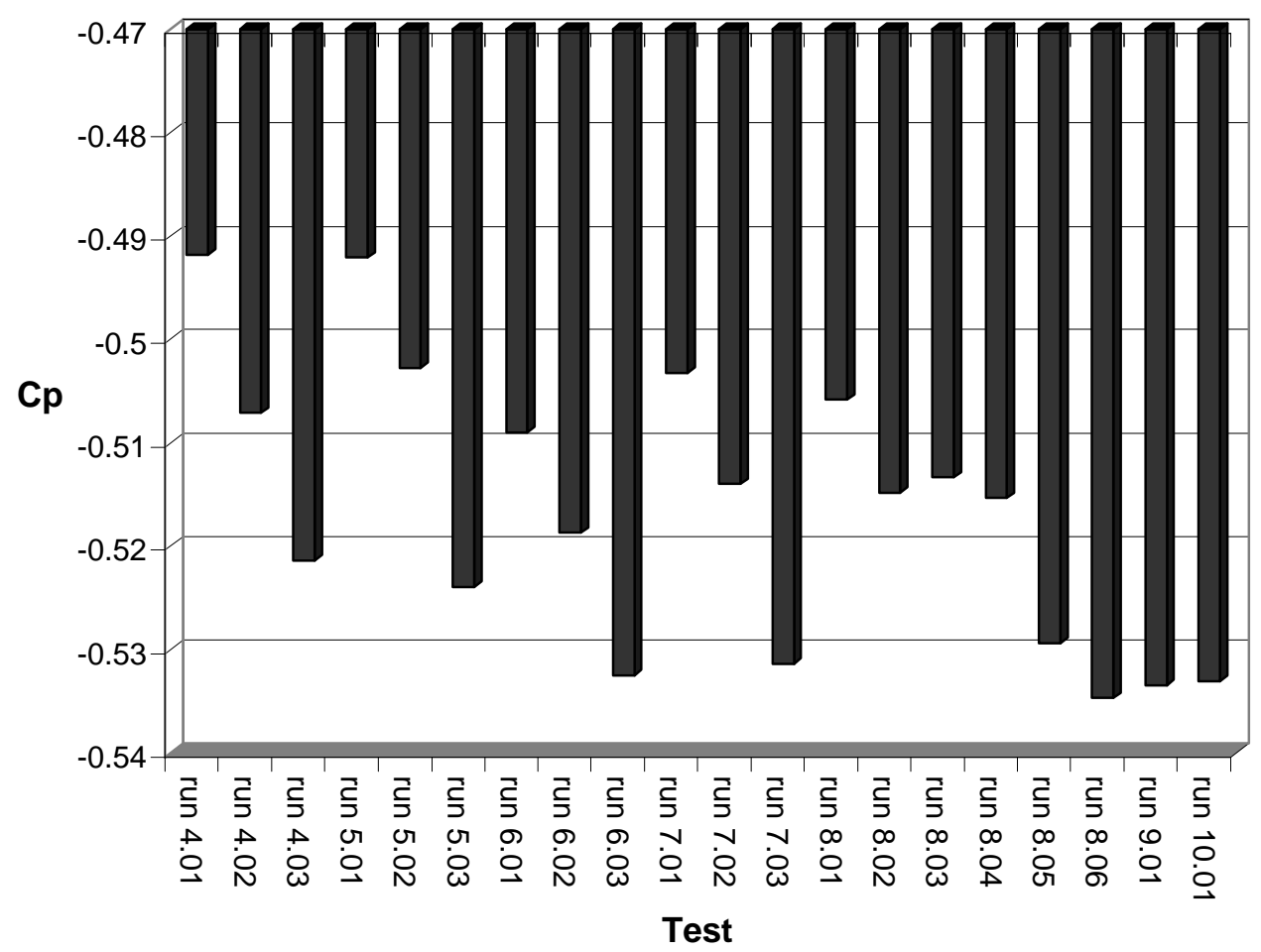

Figure A.47: Comparison of Phase II Average Pressure Coefficients for Pressure Tap \#25.

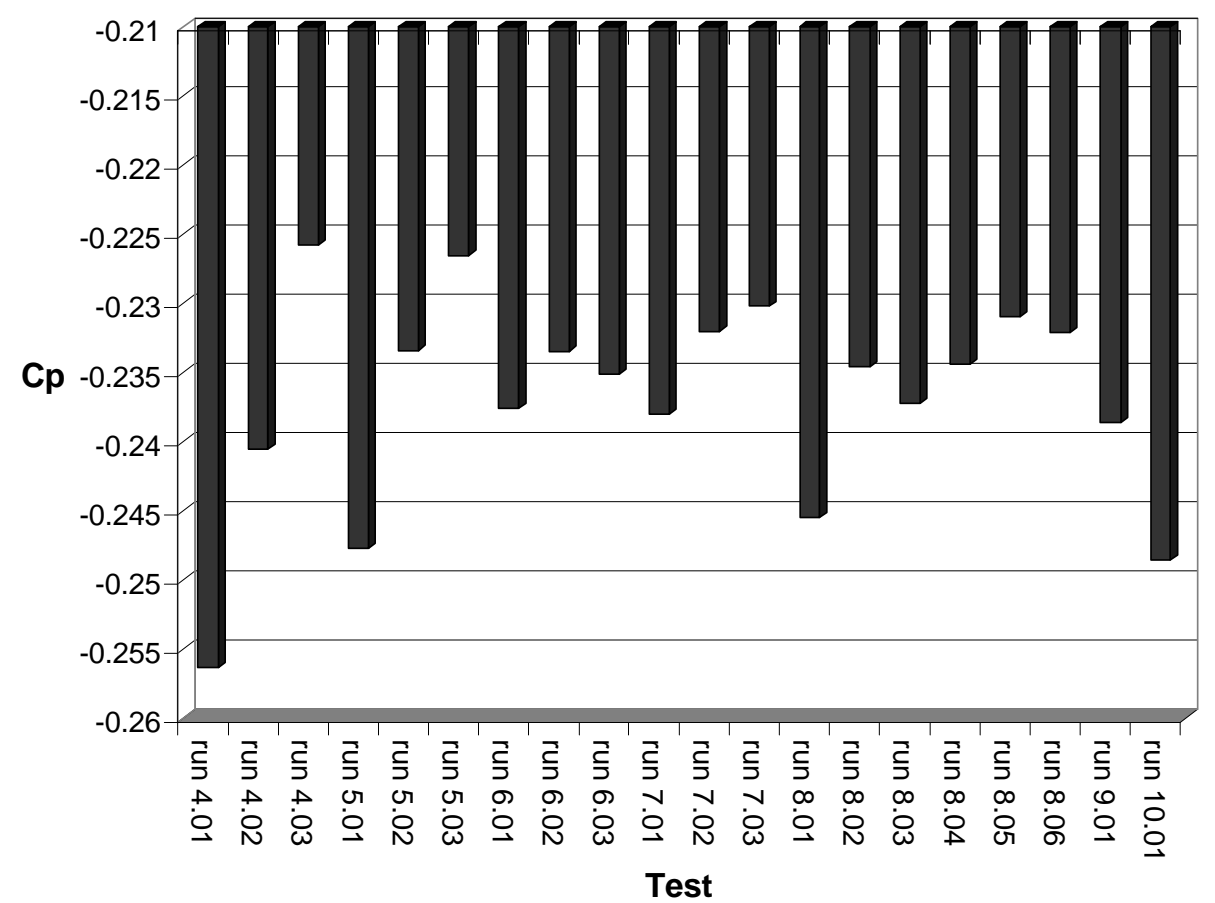

Figure A.48: Comparison of Phase II Average Pressure Coefficients for Pressure Tap $\# 27$. 


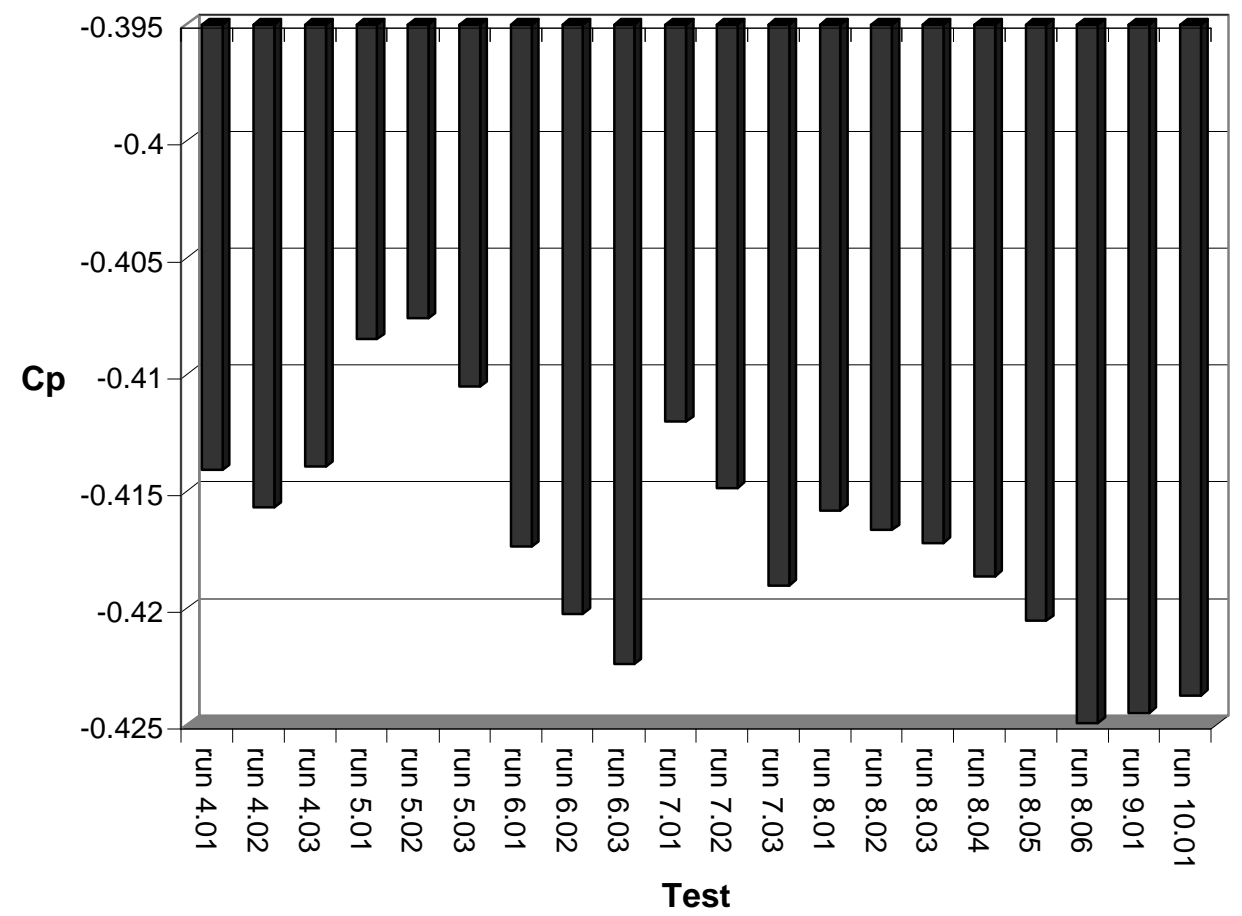

Figure A.49: Comparison of Phase II Average Pressure Coefficients for Pressure Tap \#28.

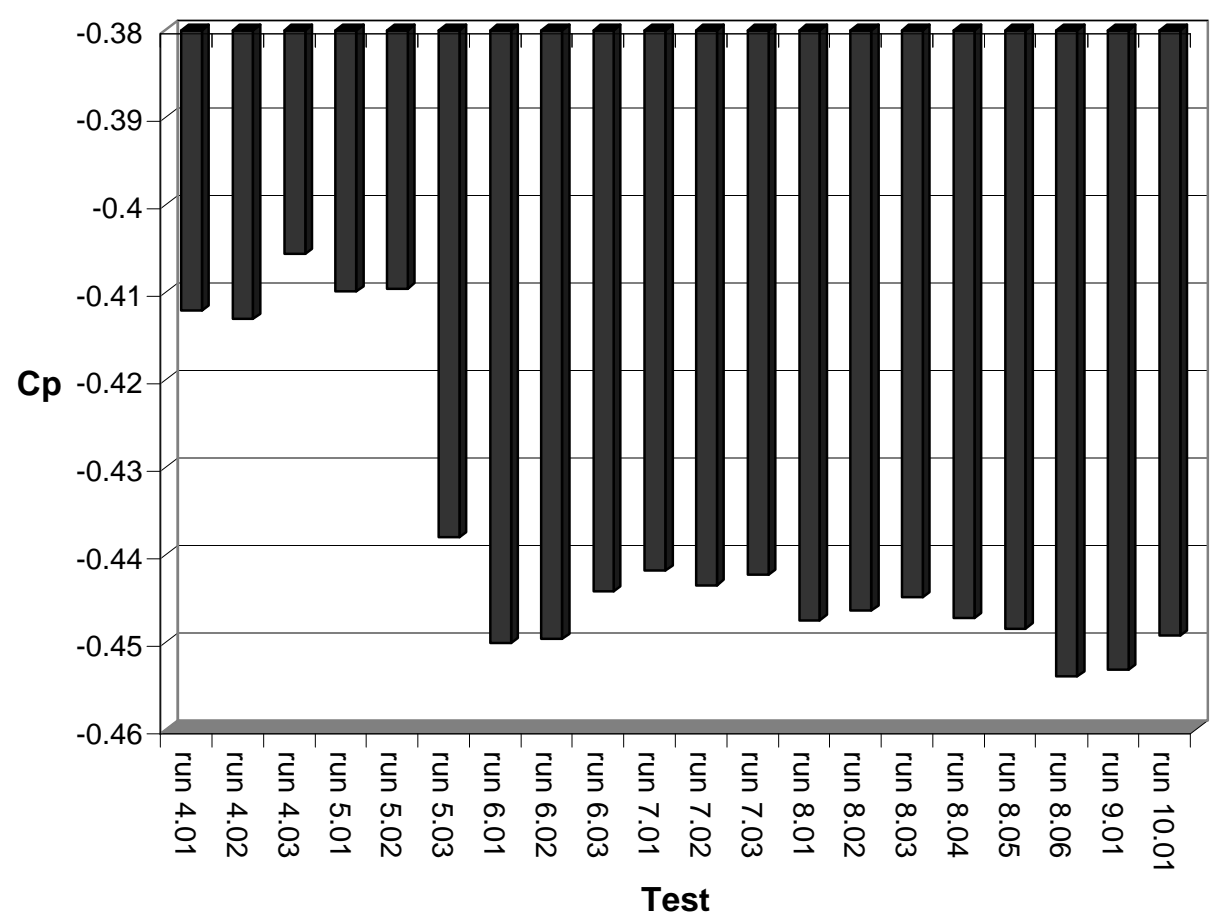

Figure A.50: Comparison of Phase II Average Pressure Coefficients for Pressure Tap \#29. 


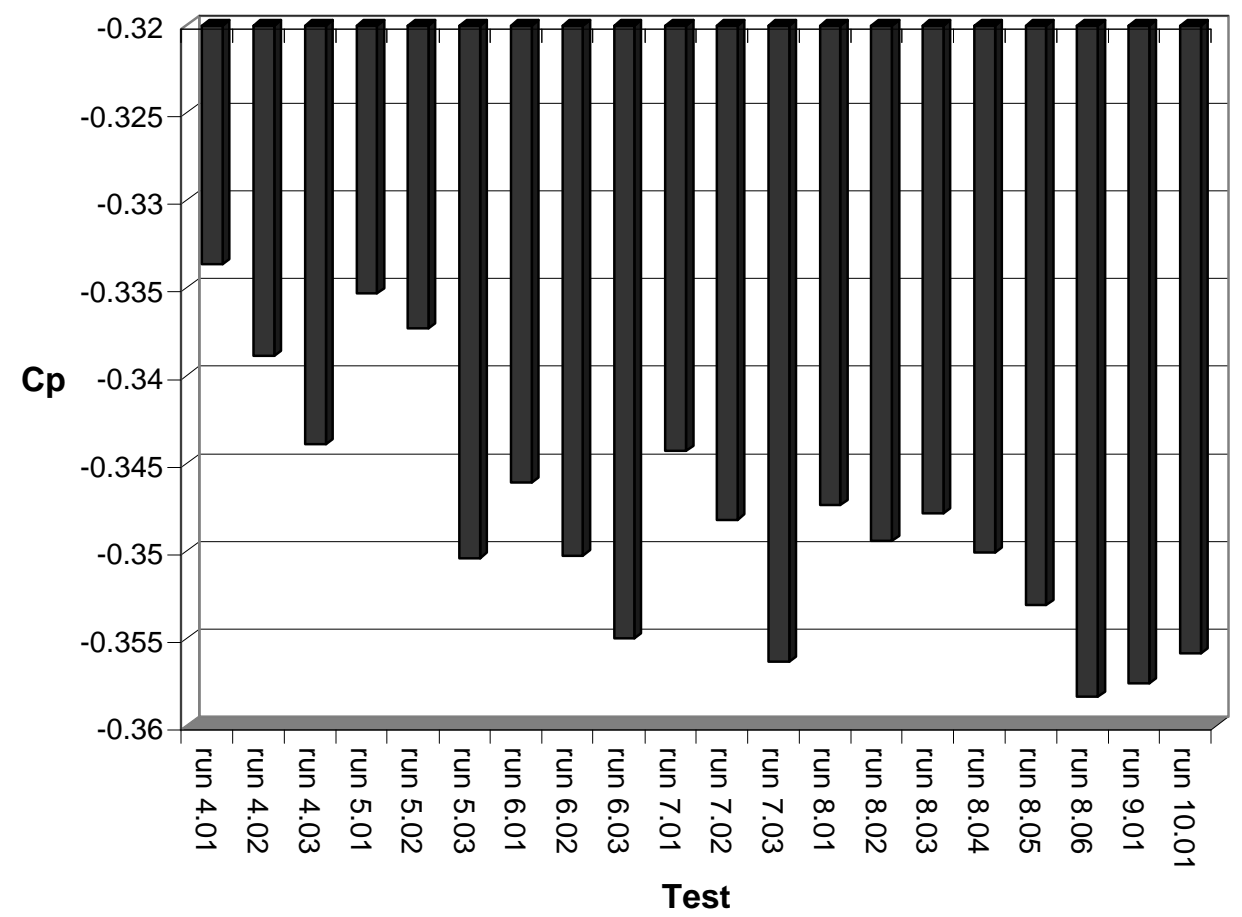

Figure A.51: Comparison of Phase II Average Pressure Coefficients for Pressure Tap \#30. 


\section{Appendix B}

LabView Programs used for Calibration and Data Acquisition. 


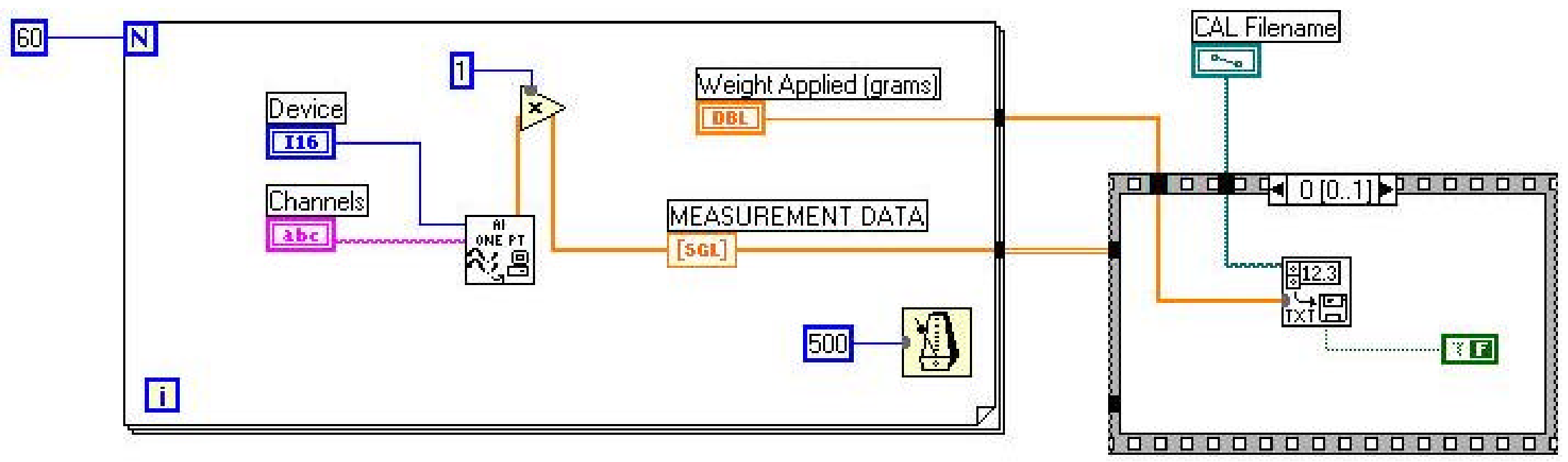

A.

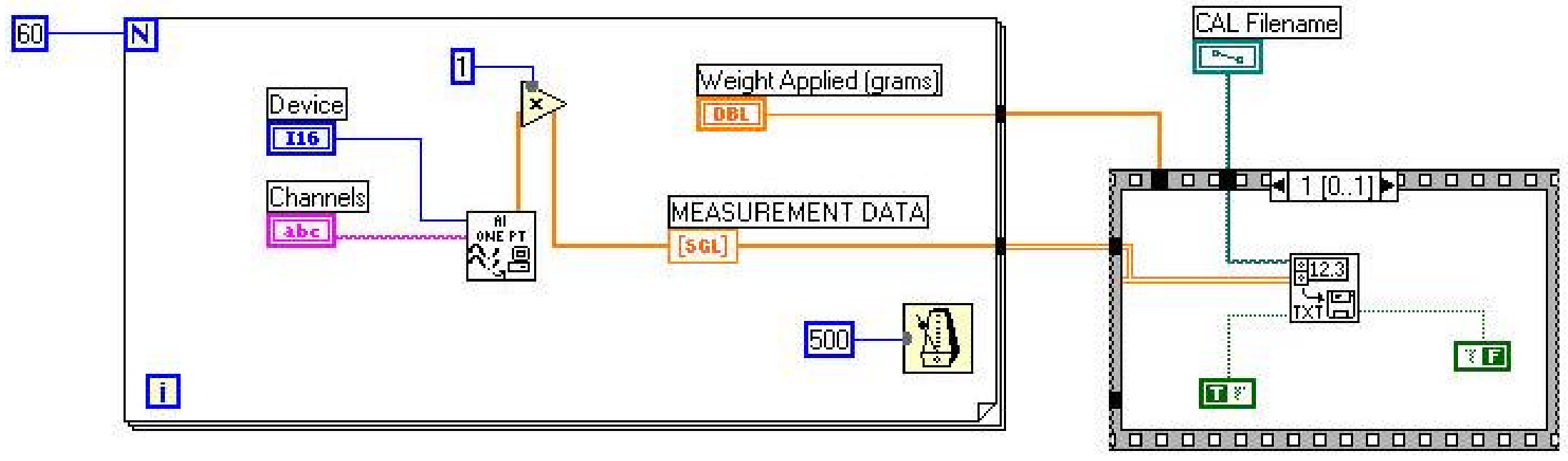

B.

Figure B.1: LabView Program used for Load Cell Calibration.
A. Step 1 of Program
B. Step 2 of Program 


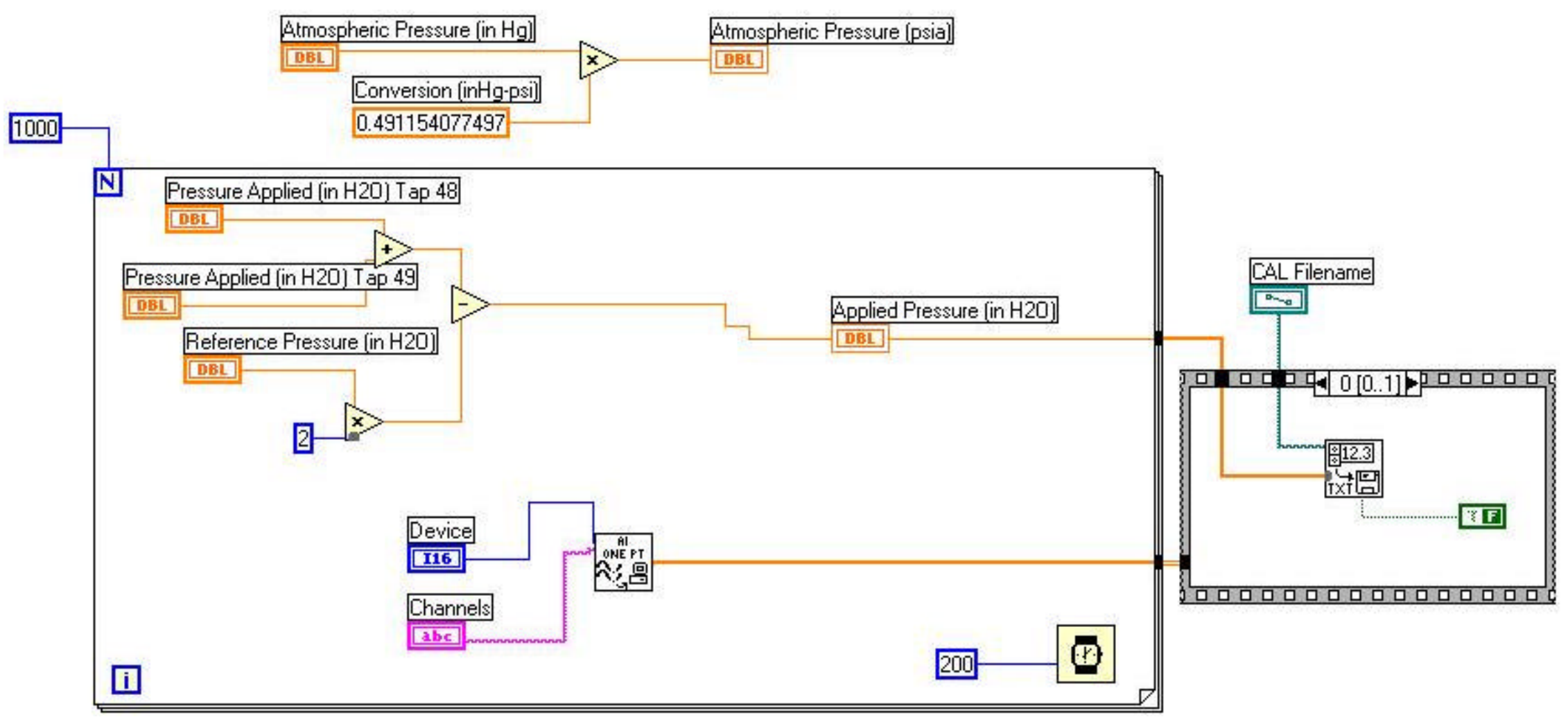

Figure B.2: Step 1 of LabView Program used for Scani-Valve Calibration. 


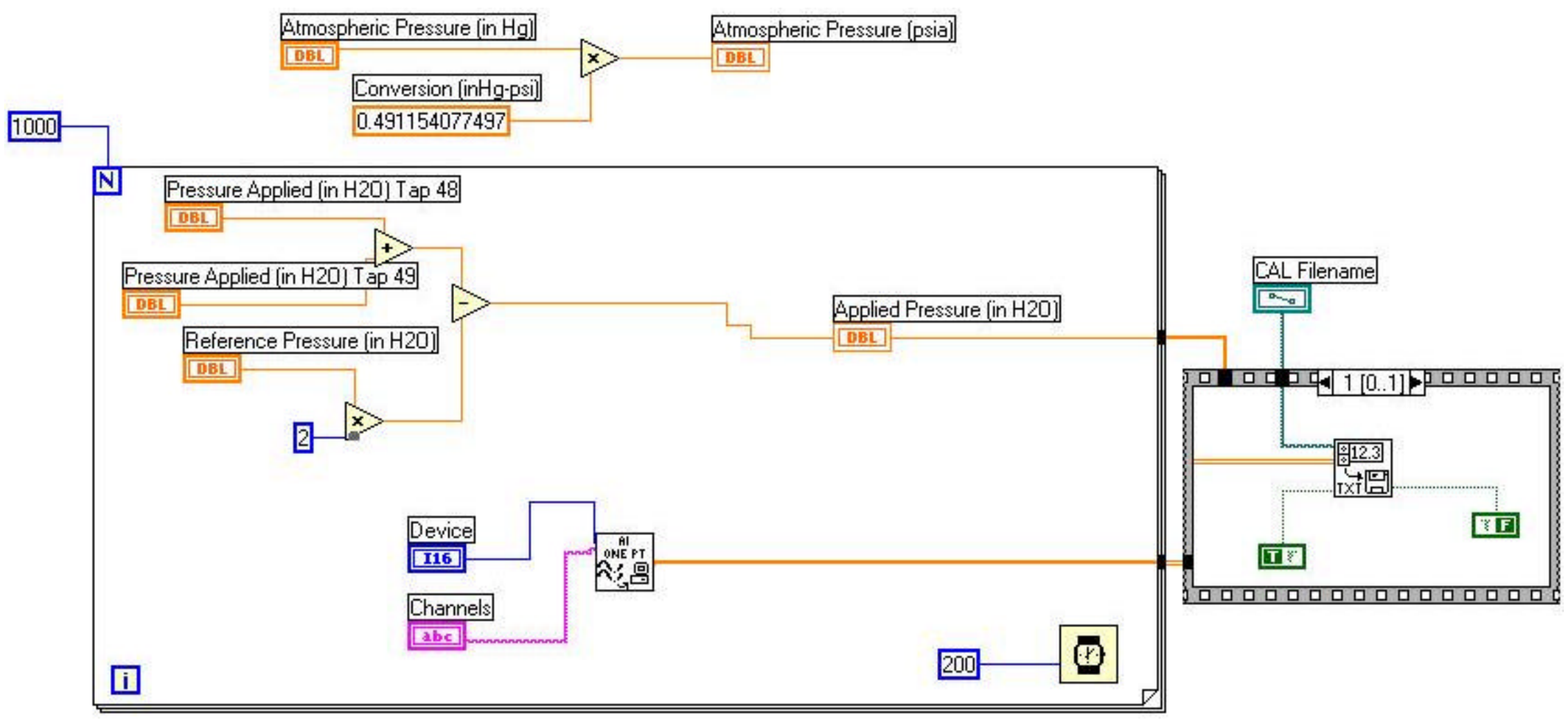

Figure B.3: Step 2 of LabView Program used for Scani-Valve Calibration. 


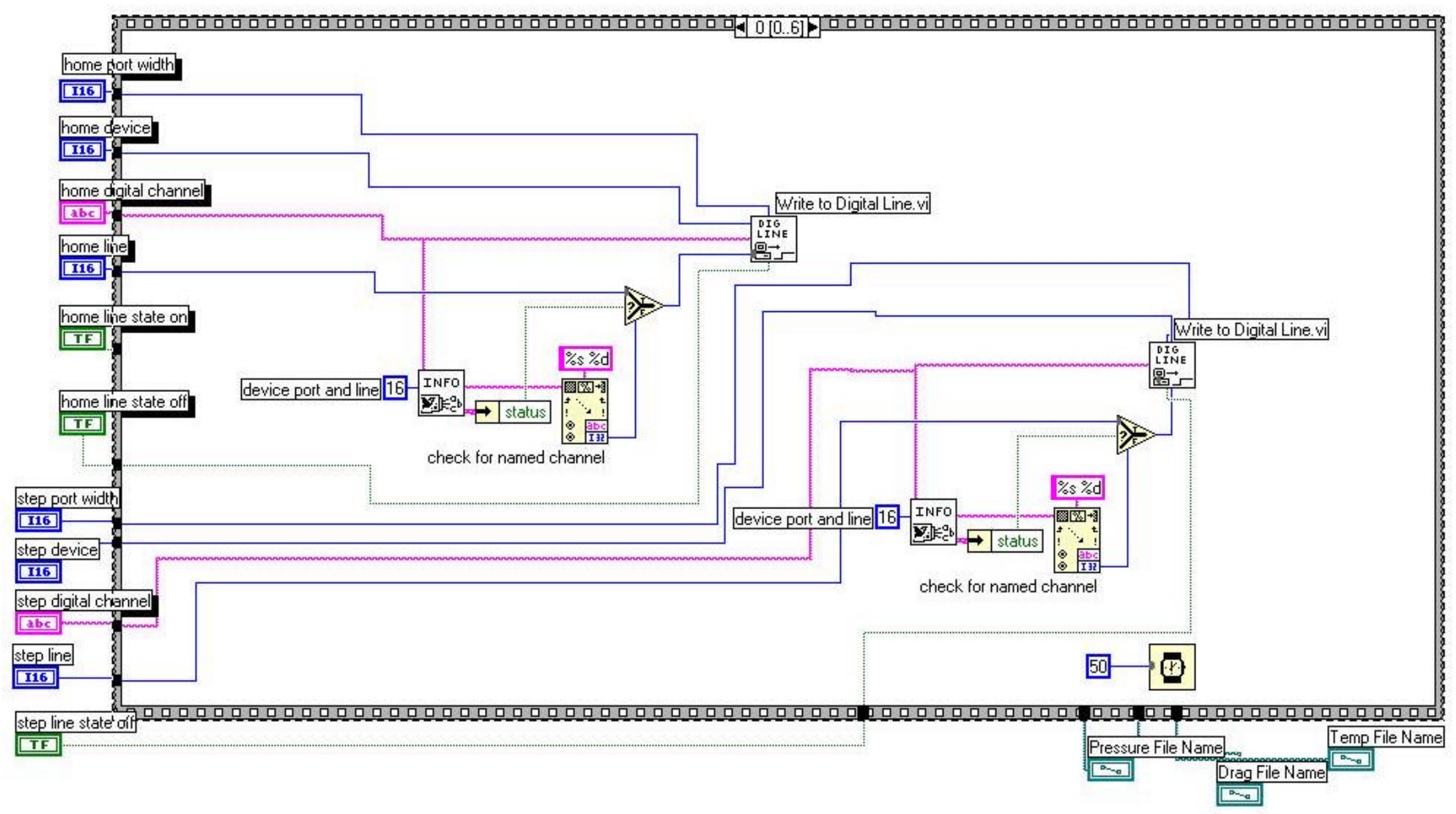

Figure B.4: Step 1 of LabView Code used for Data Acquisition. 


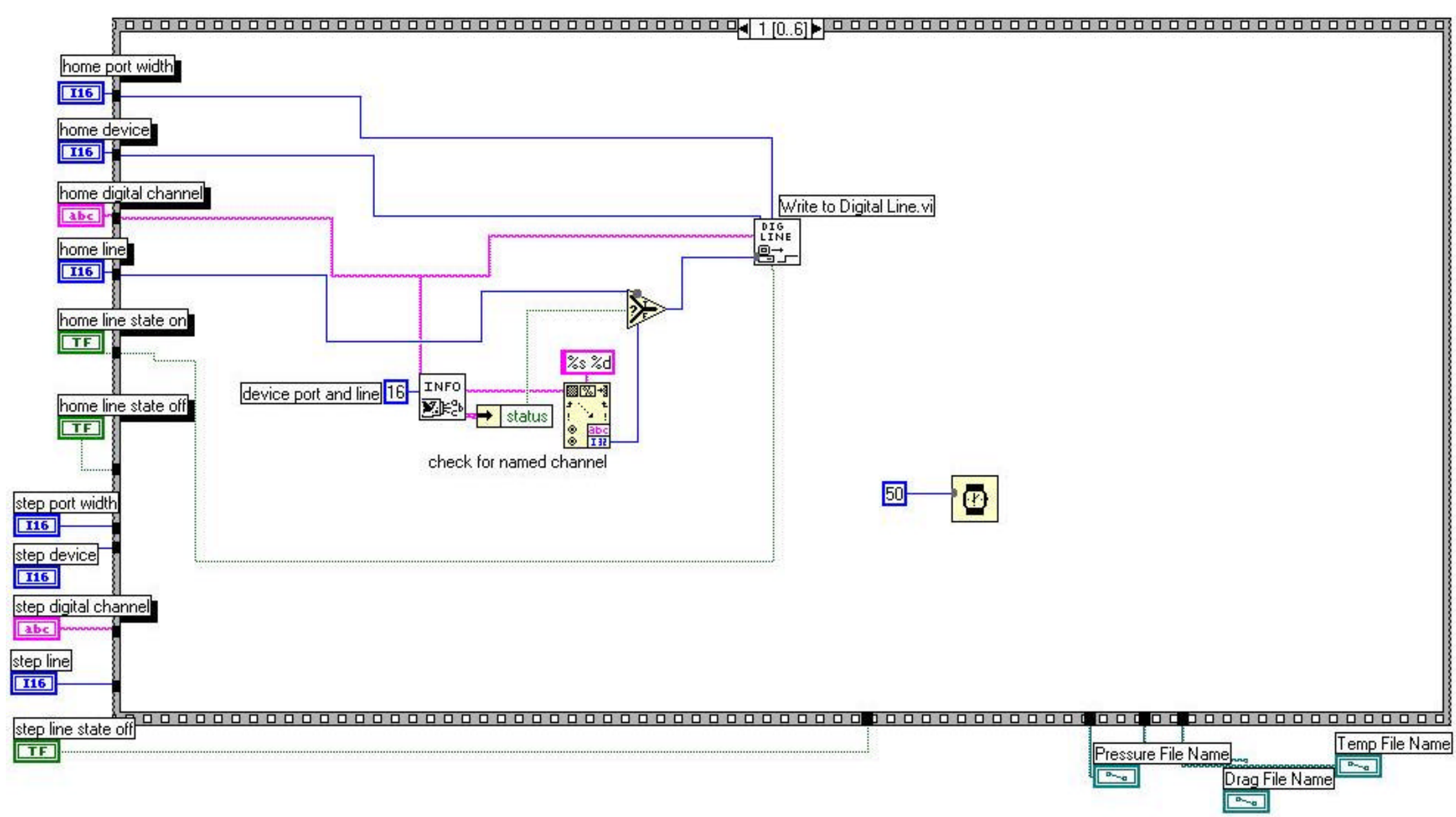

Figure B.5: Step 2 of LabView Code used for Data Acquisition. 


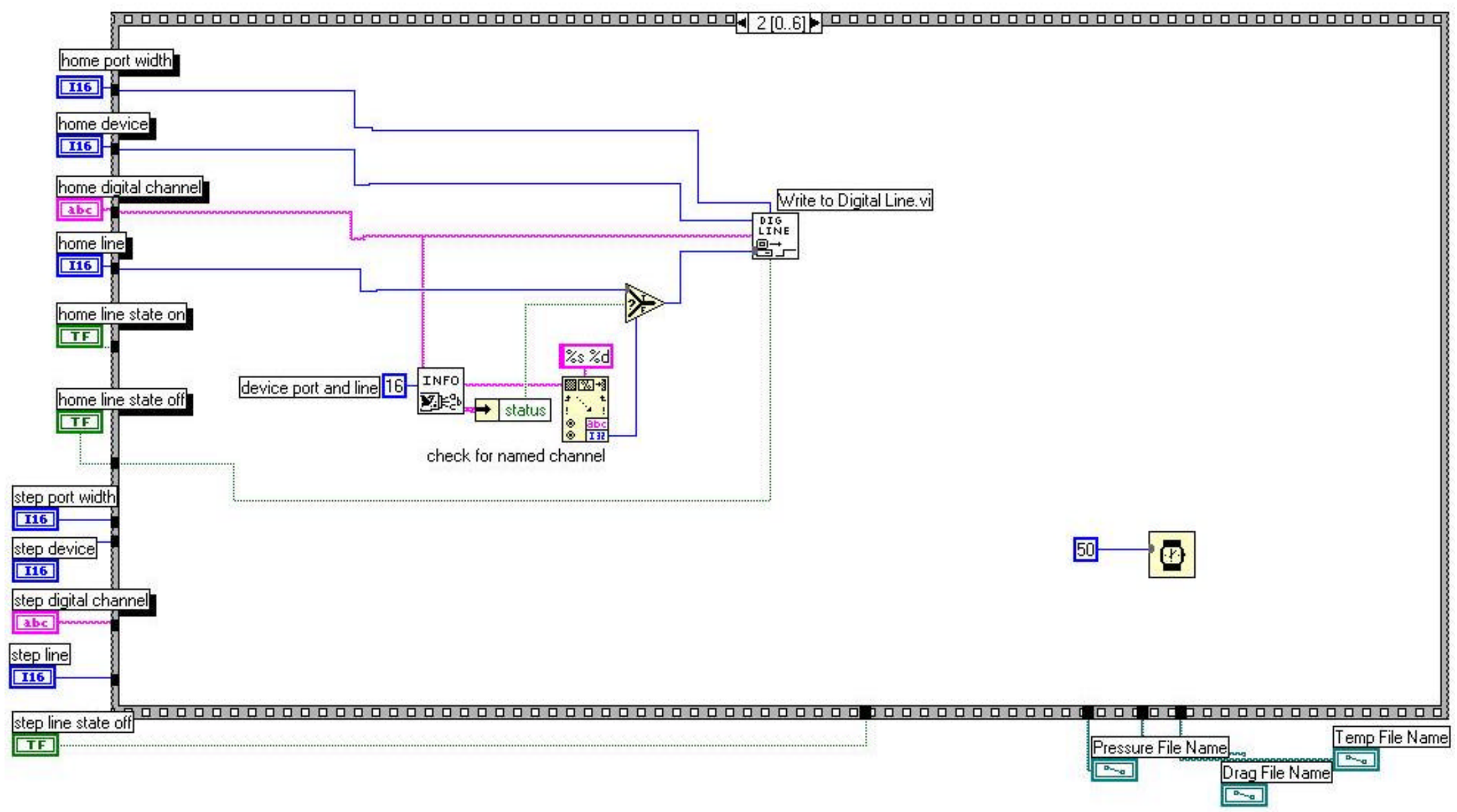

Figure B.6: Step 3 of LabView Code used for Data Acquisition. 


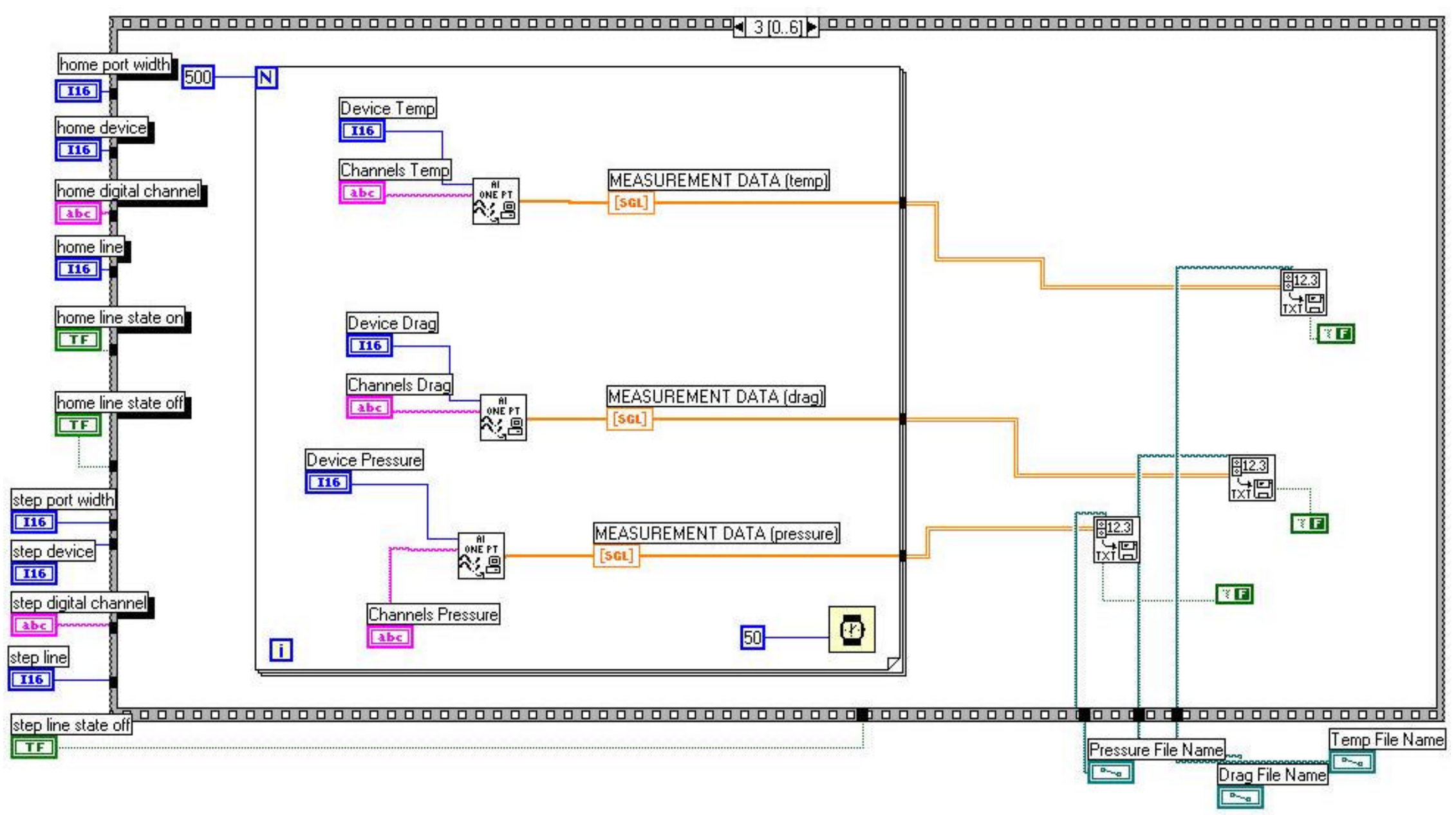

Figure B.7: Step 4 of LabView Code used for Data Acquisition. 


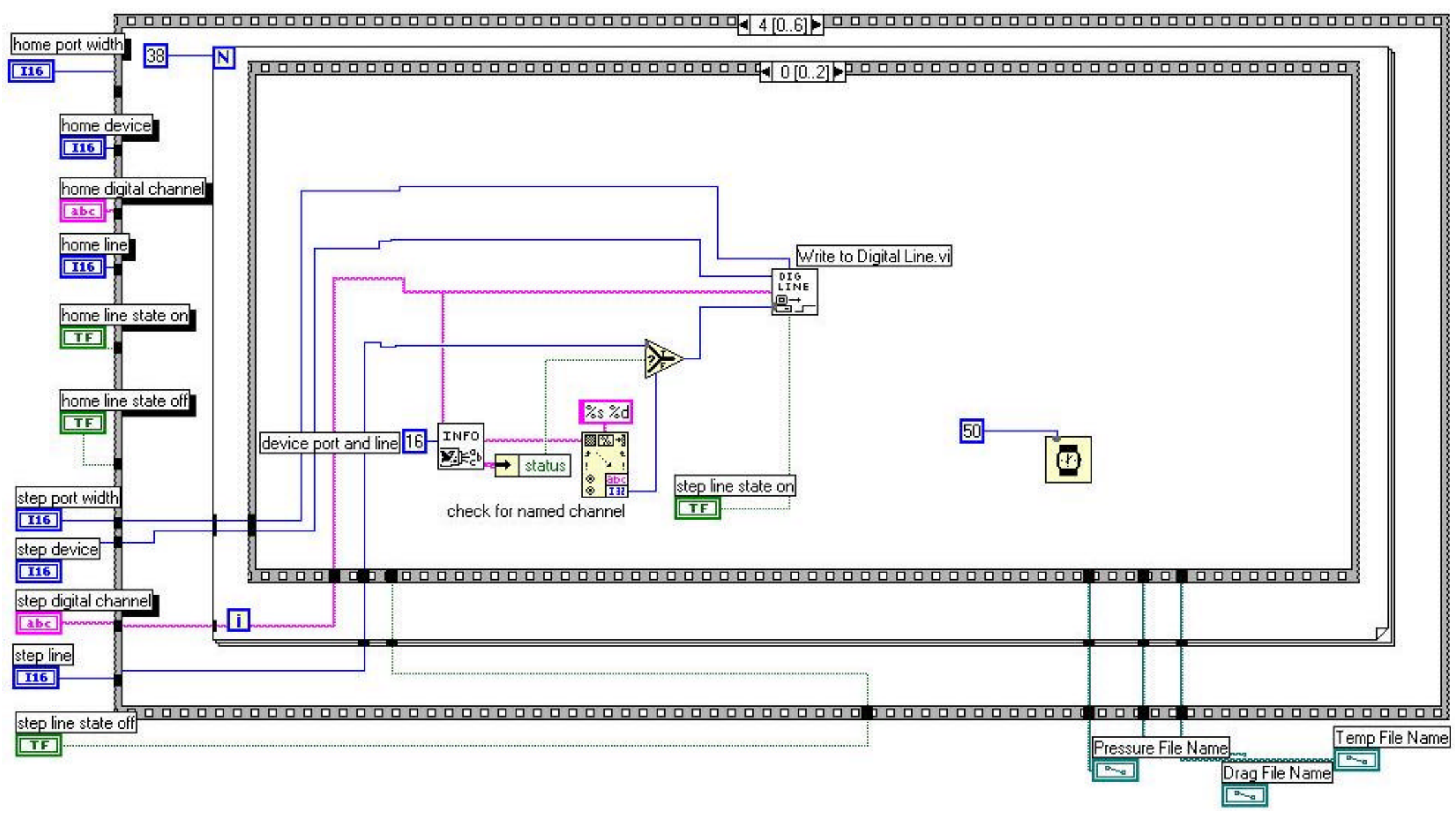

Figure B.8: Step 5 of LabView Code used for Data Acquisition. 


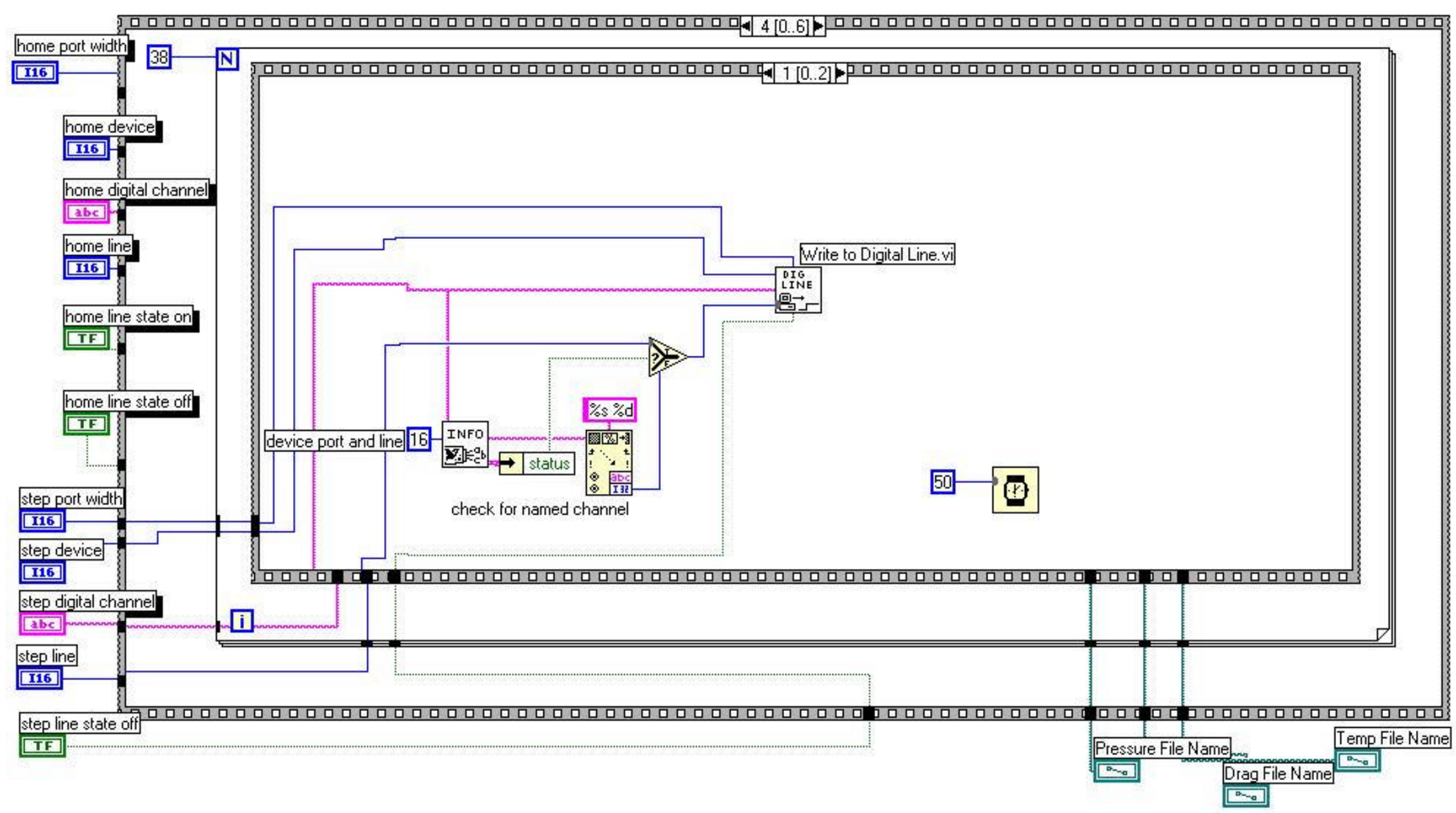

Figure B.9: Step 6 of LabView Code used for Data Acquisition. 


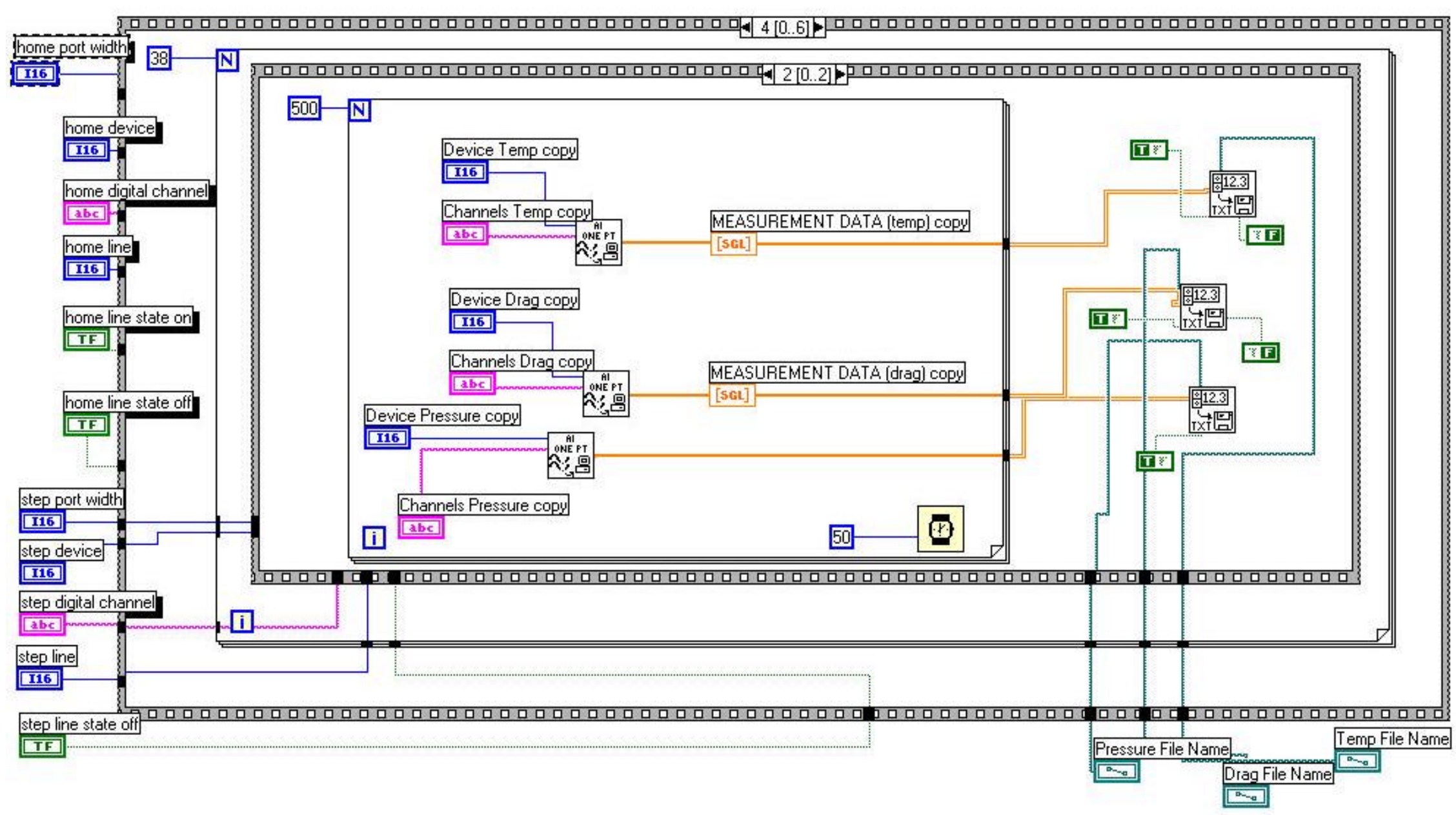

Figure B.10: Step 7 of LabView Code used for Data Acquisition. 


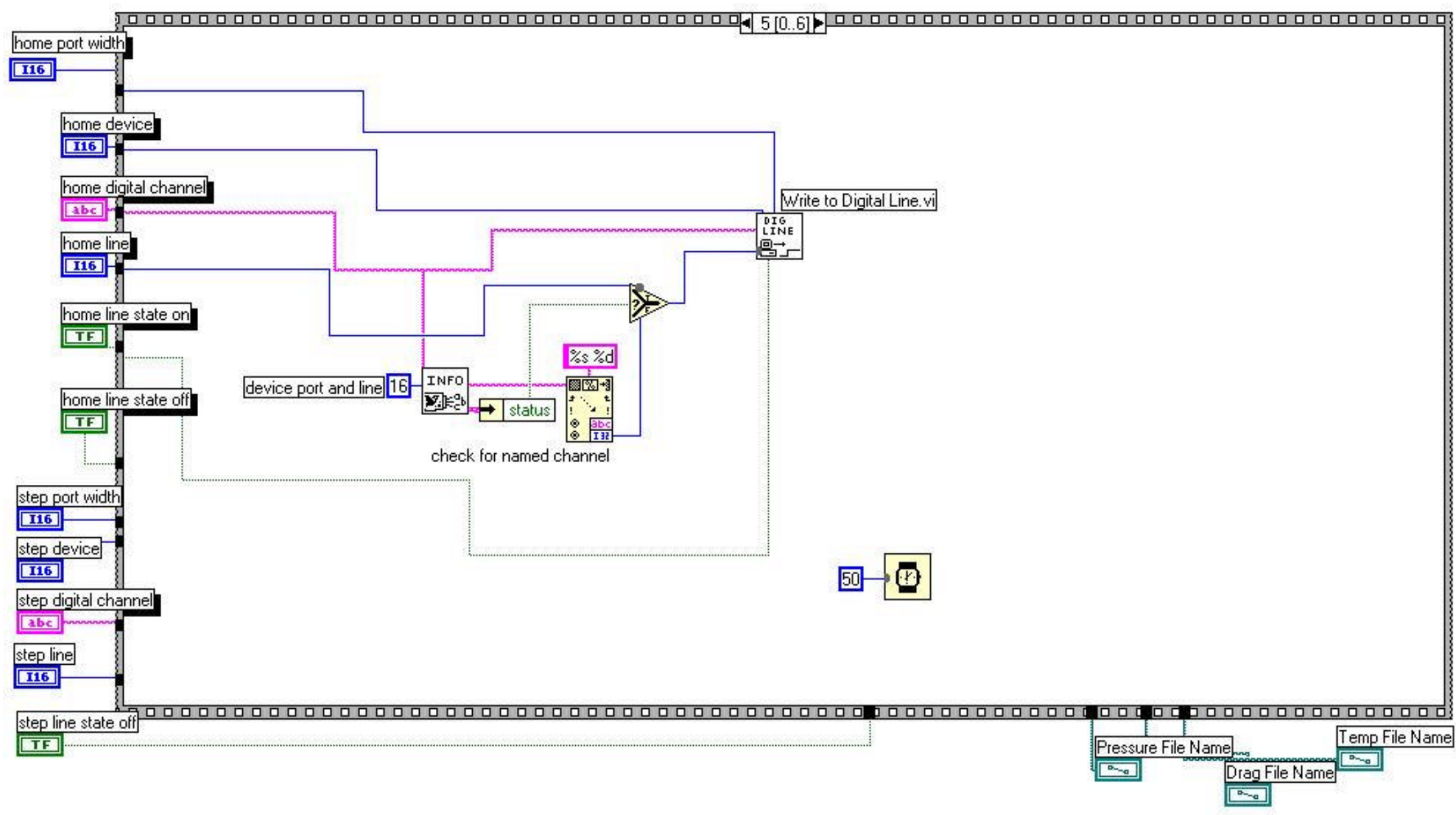

Figure B.11: Step 8 of LabView Code used for Data Acquisition. 


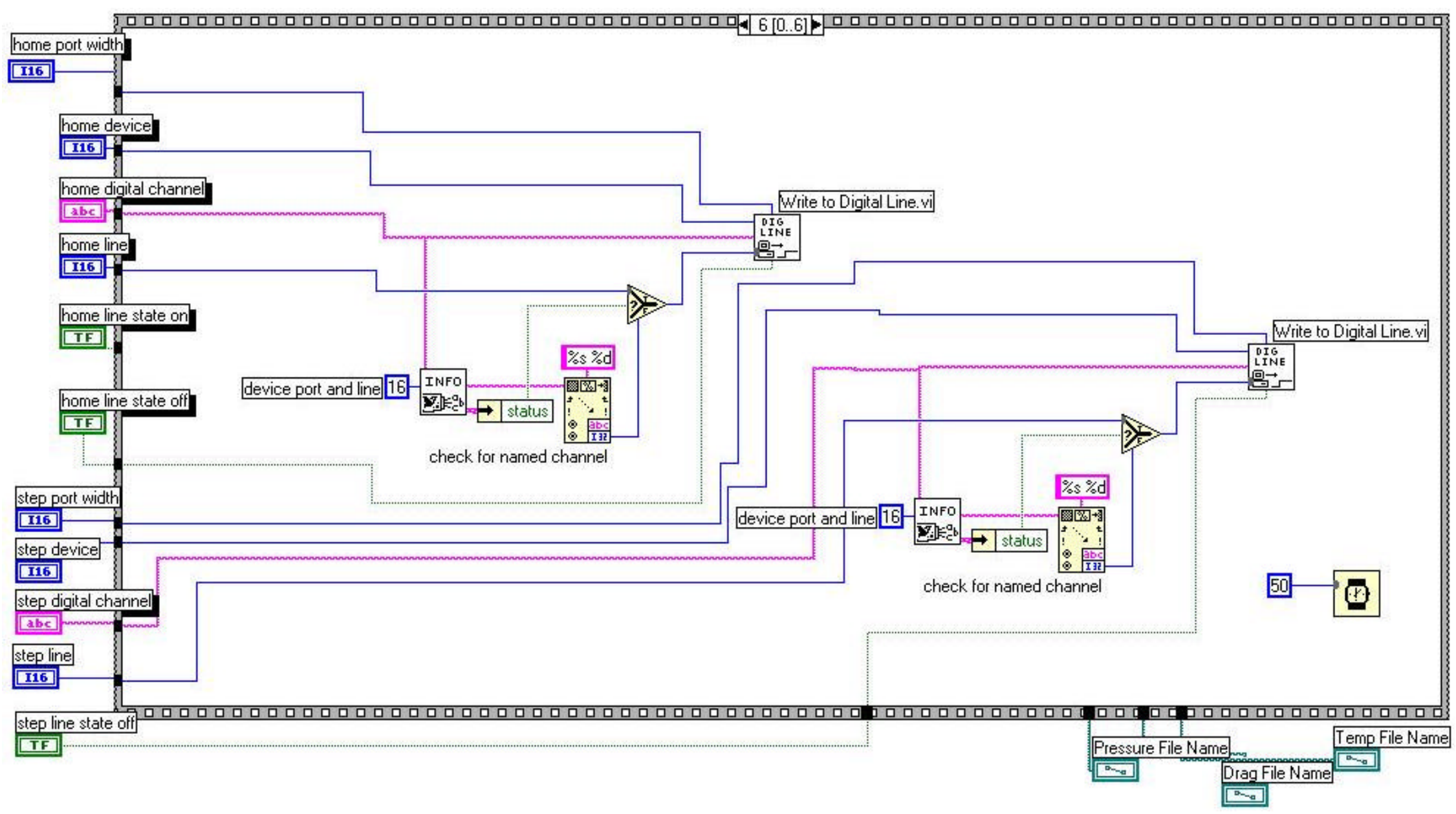

Figure B.12: Step 9 of LabView Code used for Data Acquisition. 


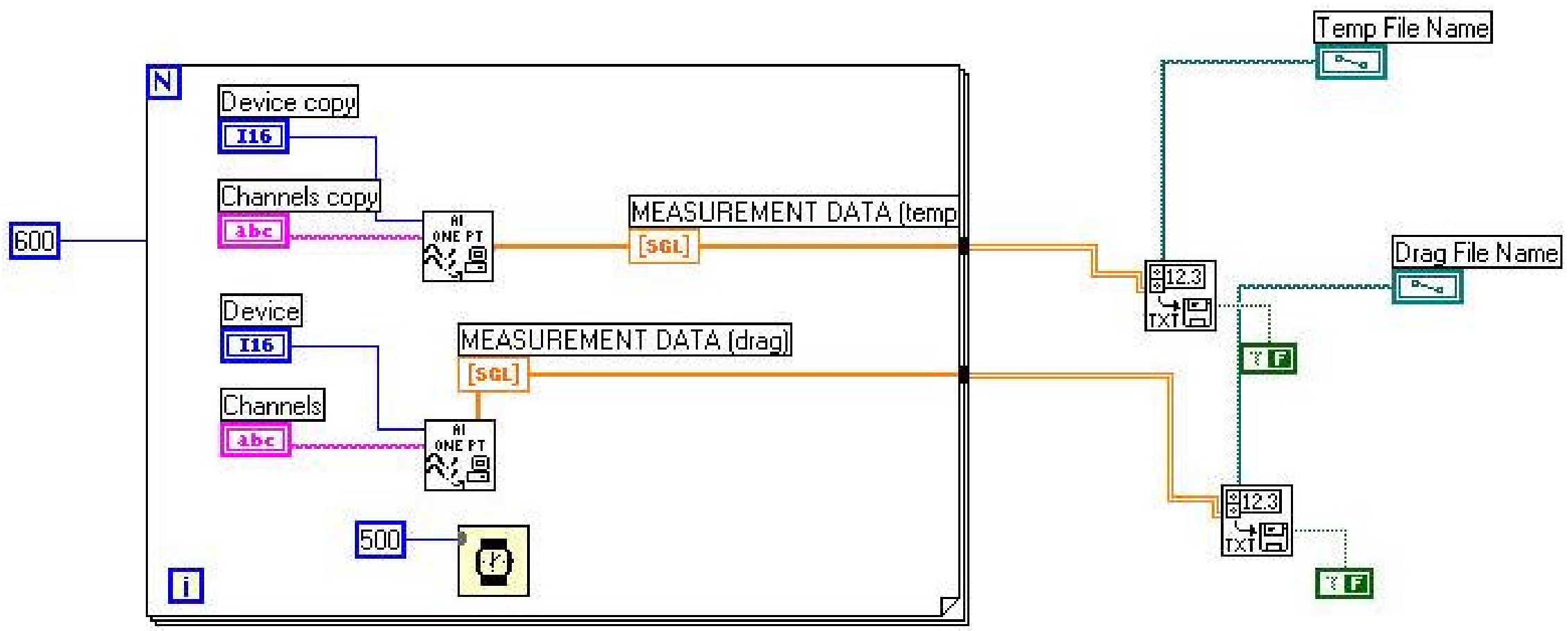

Figure B.13: LabView Code used for Data Acquisition Measuring Drag and Temperature. 


\section{Appendix C}

Log of WVU Testing, Phase I and Phase III 
Table C.1: Phase I Test Matrix

\begin{tabular}{|c|c|c|c|c|c|c|}
\hline \multirow{2}{*}{ Date } & \multirow{2}{*}{ Test } & \multicolumn{2}{|c|}{$\overline{\text { ATM }}$} & \multicolumn{3}{|c|}{ Speed (ft/s) } \\
\hline & & $\mathbf{P}$ (inHg) & $\mathrm{T}(\mathrm{oF})$ & Start & Stop & Average \\
\hline $9 / 13 / 2002$ & \multicolumn{6}{|c|}{ Calibration of Scani-Valve } \\
\hline 9/16/2002 & \multicolumn{6}{|c|}{ Second Scani-Valve calibration } \\
\hline 9/17/2002 & \multicolumn{6}{|c|}{ Model installed in test section } \\
\hline \multirow{3}{*}{$9 / 19 / 2002$} & \multicolumn{6}{|c|}{ Number of Samples $=1500$} \\
\hline & $\mathrm{B} 1 \mathrm{~T} 1$ & 29 & 75 & 55.64 & 54.64 & 55.14 \\
\hline & B1T2 & 29 & 75 & 55.8 & 55.70 & 55.75 \\
\hline \multirow{7}{*}{$9 / 20 / 2002$} & \multicolumn{6}{|c|}{ Number of Samples reduced to 1000} \\
\hline & B1T3 & 28.95 & 77 & 56.83 & 56.86 & 56.85 \\
\hline & VG1C1T1 & 28.95 & 77 & 58.02 & 57.51 & 57.77 \\
\hline & VG1C1T2 & 28.95 & 77 & 57.51 & 57.32 & 57.42 \\
\hline & VG1C1T3 & 28.95 & 77 & 58.63 & 58.12 & 58.38 \\
\hline & VG1C2T1 & 28.95 & 77 & 57.44 & 56.80 & 57.12 \\
\hline & VG1C2T2 & 28.95 & 77 & 56.8 & 56.63 & 56.72 \\
\hline \multirow{10}{*}{$9 / 23 / 2002$} & VG1C2T3 & 29.15 & 67 & 55.07 & 53.78 & 54.43 \\
\hline & VG1C3T1 & 29.15 & 67 & 53.78 & 53.47 & 53.63 \\
\hline & VG1C3T2 & 29.15 & 67 & 53.47 & 53.19 & 53.33 \\
\hline & VG1C3T3 & 29.15 & 67 & 53.19 & 52.66 & 52.93 \\
\hline & VG1C4T1 & 29.15 & 75 & 50.59 & 48.30 & 49.45 \\
\hline & VG1C4T2 & 29.15 & 75 & 48.3 & 48.10 & 48.20 \\
\hline & VG1C4T3 & 29.14 & 75 & 44.3 & 43.03 & 43.67 \\
\hline & VG1C5T1 & 29.14 & 75 & 43.25 & 42.08 & 42.67 \\
\hline & VG1C5T2 & 29.14 & 75 & 43.33 & 42.38 & 42.86 \\
\hline & VG1C5T3 & 29.14 & 75 & 42.38 & 41.63 & 42.01 \\
\hline \multirow{12}{*}{ 9/24/2002 } & B2T1 & 29.3 & 63 & 40.23 & 38.49 & 39.36 \\
\hline & B2T2 & 29.3 & 63 & 38.49 & 53.22 & 45.86 \\
\hline & \multicolumn{6}{|c|}{ Manometer Replaced due to inaccurate readings, re-run VG1C4 and VG1C5. } \\
\hline & B2T3 & 29.19 & 76 & 52 & 51.64 & 51.82 \\
\hline & B2T4 & 29.19 & 76 & 51.64 & 51.28 & 51.46 \\
\hline & $\mathrm{B2T5}$ & 29.19 & 76 & 51.28 & 51.28 & 51.28 \\
\hline & VG2C1T1 & 29.2 & 76 & 54.43 & 54.43 & 54.43 \\
\hline & VG2C1T2 & 29.2 & 76 & 54.43 & 54.09 & 54.26 \\
\hline & VG2C1T3 & 29.2 & 76 & 54.09 & 54.09 & 54.09 \\
\hline & VG2C2T1 & 29.2 & 76 & 55.11 & 55.11 & 55.11 \\
\hline & VG2C2T2 & 29.2 & 76 & 55.11 & 55.11 & 55.11 \\
\hline & VG2C2T3 & 29.2 & 76 & 55.11 & 54.94 & 55.03 \\
\hline
\end{tabular}


Table C.2: Phase I Test Matrix, Continued

\begin{tabular}{|c|c|c|c|c|c|c|}
\hline \multirow{2}{*}{ Date } & \multirow{2}{*}{ Test } & \multicolumn{2}{|c|}{ ATM } & \multicolumn{3}{|c|}{ Speed (ft/s) } \\
\hline & & $\mathbf{P}$ (inHg) & $\mathrm{T}(\mathrm{OF})$ & Start & Stop & Average \\
\hline \multirow{13}{*}{$9 / 25 / 2002$} & VG2C3T1 & 29.24 & 67 & 55.78 & 55.11 & 55.45 \\
\hline & VG2C3T2 & 29.24 & 67 & 55.11 & 55.11 & 55.11 \\
\hline & VG2C3T3 & 29.24 & 67 & 55.11 & 55.11 & 55.11 \\
\hline & \multicolumn{6}{|c|}{ RPM Sensor stopped working proceeded testing with permission } \\
\hline & VG2C4T1 & 29.24 & 67 & 55.11 & 55.11 & 55.11 \\
\hline & VG2C4T2 & 29.24 & 67 & 55.11 & 55.11 & 55.11 \\
\hline & VG2C4T3 & 29.24 & 67 & 54.77 & 55.11 & 54.94 \\
\hline & VG2C5T1 & 29.24 & 67 & 55.11 & 54.43 & 54.77 \\
\hline & VG2C5T2 & 29.24 & 67 & 54.43 & 54.43 & 54.43 \\
\hline & VG2C5T3 & 29.24 & 67 & 54.43 & 54.43 & 54.43 \\
\hline & B3T1 & 29.24 & 67 & 54.77 & 54.77 & 54.77 \\
\hline & B3T2 & 29.24 & 67 & 54.77 & 54.77 & 54.77 \\
\hline & B3T3 & 29.24 & 67 & 54.77 & 54.77 & 54.77 \\
\hline \multirow{15}{*}{$9 / 26 / 2002$} & VG3C1T1 & 29.04 & 67 & 55.11 & 54.77 & 54.94 \\
\hline & VG3C1T2 & 29.04 & 67 & 54.77 & 54.77 & 54.77 \\
\hline & VG3C1T3 & 29.04 & 67 & 54.77 & 54.77 & 54.77 \\
\hline & VG3C2T1 & 28.98 & 68 & 55.11 & 54.77 & 54.94 \\
\hline & VG3C2T2 & 28.98 & 68 & 54.77 & 54.77 & 54.77 \\
\hline & VG3C2T3 & 28.98 & 68 & 54.77 & 54.43 & 54.60 \\
\hline & VG3C3T1 & 28.98 & 68 & 54.77 & 54.43 & 54.60 \\
\hline & VG3C3T2 & 28.98 & 68 & 54.43 & 54.43 & 54.43 \\
\hline & VG3C3T3 & 28.98 & 68 & 54.43 & 54.43 & 54.43 \\
\hline & VG3C4T1 & 28.98 & 68 & 54.43 & 54.09 & 54.26 \\
\hline & VG3C4T2 & 28.98 & 68 & 54.09 & 54.09 & 54.09 \\
\hline & VG3C4T3 & 28.98 & 68 & 54.09 & 54.09 & 54.09 \\
\hline & VG3C5T1 & 28.94 & 68 & 55.11 & 55.11 & 55.11 \\
\hline & VG3C5T2 & 28.94 & 68 & 55.11 & 55.11 & 55.11 \\
\hline & VG3C5T3 & 28.94 & 68 & 55.11 & 54.94 & 55.03 \\
\hline \multirow{16}{*}{ 9/27/2002 } & VG3M1 & 28.62 & 68 & 54.77 & 54.43 & 54.60 \\
\hline & & \multicolumn{5}{|c|}{ Config 5 with VG added to the handle bar ends } \\
\hline & VG3M2 & 28.62 & 68 & 54.43 & 54.43 & 54.43 \\
\hline & & \multicolumn{5}{|c|}{ VG3M1 with upper fairing VG's moved an inch forward } \\
\hline & VG3M3 & 28.62 & 68 & 54.77 & 54.77 & 54.77 \\
\hline & & \multicolumn{5}{|c|}{ VG3M2 with lower fairing VG's moved aft an inch } \\
\hline & VG3M4 & 28.62 & 68 & 54.43 & 54.43 & 54.43 \\
\hline & \multicolumn{6}{|c|}{ VG3M3 with upper fairing VG's moved toward centerline an inch } \\
\hline & VG3M5 & 28.62 & 68 & 55.11 & 55.28 & 55.20 \\
\hline & & \multicolumn{5}{|c|}{ VG3M4 with upper fairing VG's forward an addition inch } \\
\hline & VG1C4T1 re-run & 28.62 & 68 & 54.43 & 53.75 & 54.09 \\
\hline & VG1C4T2 re-run & 28.62 & 68 & 53.75 & 54.09 & 53.92 \\
\hline & VG1C4T3 re-run & 28.62 & 68 & 54.09 & 54.09 & 54.09 \\
\hline & VG1C5T1 re-run & 28.58 & 68 & 53.4 & 53.05 & 53.23 \\
\hline & VG1C5T2 re-run & 28.58 & 68 & 53.4 & 53.40 & 53.40 \\
\hline & VG1C5T3 re-run & 28.58 & 68 & 53.4 & 53.40 & 53.40 \\
\hline \multirow{5}{*}{$9 / 28 / 2002$} & B4T1 & 29.02 & 68 & 54.43 & 54.43 & 54.43 \\
\hline & B4T2 & 29.02 & 68 & 54.43 & 54.43 & 54.43 \\
\hline & B4T1M & 29.02 & 68 & 55.11 & 55.11 & 55.11 \\
\hline & B4T2M & 29.02 & 68 & 55.11 & 55.11 & 55.11 \\
\hline & B4T3M & 29.02 & 68 & 55.11 & 54.94 & 55.03 \\
\hline
\end{tabular}


Table C.3: Phase III Test Matrix

\begin{tabular}{|c|c|c|c|c|c|c|c|}
\hline \multirow{2}{*}{ Date } & \multirow{2}{*}{ Test } & \multicolumn{2}{|c|}{ ATM } & \multicolumn{3}{|c|}{ Manometer Height (inH2O) } & \multirow{2}{*}{\begin{tabular}{|l|} 
Speed \\
$\mathrm{ft} / \mathrm{s}$
\end{tabular}} \\
\hline & & $P($ inHg) & $\mathrm{T}(\mathrm{oF})$ & Start & Stop & Average & \\
\hline \multirow{3}{*}{ 10/15/2002 } & OB1T1 & 28.98 & 70 & 0.71 & 0.65 & 0.68 & 50.18 \\
\hline & OB1T2 & 28.98 & 70 & 0.65 & 0.66 & 0.66 & 49.25 \\
\hline & \multicolumn{3}{|c|}{ Readings taken with no velocity } & & & & \\
\hline \multirow{30}{*}{$10 / 16 / 2002$} & VG1C1T1 & 28.72 & 72 & 0.69 & 0.69 & 0.69 & 50.55 \\
\hline & VG1C1T2 & 28.72 & 72 & 0.69 & 0.69 & 0.69 & 50.55 \\
\hline & VG1C1T3 & 28.72 & 72 & 0.69 & 0.69 & 0.69 & 50.55 \\
\hline & \begin{tabular}{|l} 
VG1C1T4 \\
\end{tabular} & 28.72 & 72 & 0.69 & 0.69 & 0.69 & 50.55 \\
\hline & VG1C2T1 & 28.72 & 72 & 0.69 & 0.71 & 0.70 & 50.92 \\
\hline & VG1C2T2 & 28.72 & 72 & 0.71 & 0.71 & 0.71 & 51.28 \\
\hline & VG1C2T3 & 28.72 & 72 & 0.7 & 0.71 & 0.71 & 51.10 \\
\hline & VG1C2T4 & 28.72 & 72 & 0.71 & 0.7 & 0.71 & 51.10 \\
\hline & OB1T3 & 28.72 & 72 & 0.73 & 0.75 & 0.74 & 52.35 \\
\hline & OB1T4 & 28.72 & 72 & 0.75 & 0.75 & 0.75 & 52.70 \\
\hline & OVG2C3T1 & 28.72 & 72 & 0.75 & 0.75 & 0.75 & 52.70 \\
\hline & OVG2C3T2 & 28.72 & 72 & 0.75 & 0.75 & 0.75 & 52.70 \\
\hline & OVG2C3T3 & 28.72 & 72 & 0.75 & 0.75 & 0.75 & 52.70 \\
\hline & OVG2C3T4 & 28.72 & 72 & 0.75 & 0.75 & 0.75 & 52.70 \\
\hline & OVG2C4T1 & 28.72 & 72 & 0.76 & 0.76 & 0.76 & 53.05 \\
\hline & OVG2C4T2 & 28.72 & 72 & 0.76 & 0.76 & 0.76 & 53.05 \\
\hline & OVG2C4T3 & 28.72 & 72 & 0.76 & 0.76 & 0.76 & 53.05 \\
\hline & OVG2C4T4 & 28.72 & 72 & 0.76 & 0.76 & 0.76 & 53.05 \\
\hline & OVG3C4T1 & 28.72 & 72 & 0.71 & 0.7 & 0.71 & 51.10 \\
\hline & OVG3C4T2 & 28.72 & 72 & 0.7 & 0.7 & 0.70 & 50.92 \\
\hline & OVG3C4T3 & 28.72 & 72 & 0.7 & 0.7 & 0.70 & 50.92 \\
\hline & OVG3C4T4 & 28.72 & 72 & 0.7 & 0.7 & 0.70 & 50.92 \\
\hline & OVG3C5T1 & 28.72 & 72 & 0.72 & 0.71 & 0.72 & 51.46 \\
\hline & OVG3C5T2 & 28.72 & 72 & 0.71 & 0.71 & 0.71 & 51.28 \\
\hline & OVG3C5T3 & 28.72 & 72 & 0.71 & 0.71 & 0.71 & 51.28 \\
\hline & OVG3C5T4 & 28.72 & 72 & 0.71 & 0.71 & 0.71 & 51.28 \\
\hline & OVG3M1T1 & 28.72 & 72 & 0.73 & 0.74 & 0.74 & 52.17 \\
\hline & OVG3M1T2 & 28.72 & 72 & 0.74 & 0.73 & 0.74 & 52.17 \\
\hline & OVG3M1T3 & 28.72 & 72 & 0.73 & 0.74 & 0.74 & 52.17 \\
\hline & OVG3M1T4 & 28.72 & 72 & 0.74 & 0.73 & 0.74 & 52.17 \\
\hline \multirow{14}{*}{ 10/17/2002 } & OVG3P1T1 & 28.93 & 74 & 0.72 & 0.72 & 0.72 & 51.64 \\
\hline & OVG3P1T2 & 28.93 & 74 & 0.72 & 0.71 & 0.72 & 51.46 \\
\hline & OVG3P1T3 & 28.93 & 74 & 0.71 & 0.72 & 0.72 & 51.46 \\
\hline & OVG3P1T4 & 28.93 & 74 & 0.72 & 0.71 & 0.72 & 51.46 \\
\hline & OVG3P2T1 & 28.93 & 74 & 0.73 & 0.74 & 0.74 & 52.17 \\
\hline & OVG3P2T2 & 28.93 & 74 & 0.74 & 0.74 & 0.74 & 52.35 \\
\hline & OVG3P2T3 & 28.93 & $\overline{74}$ & 0.74 & 0.74 & 0.74 & 52.35 \\
\hline & OVG3P2T4 & 28.93 & $\overline{74}$ & 0.74 & 0.74 & 0.74 & 52.35 \\
\hline & OVG3P3T1 & 28.93 & 74 & 0.72 & 0.72 & 0.72 & 51.64 \\
\hline & OVG3P3T2 & 28.93 & $\overline{74}$ & 0.72 & 0.72 & 0.72 & 51.64 \\
\hline & OVG3P3T3 & 28.93 & 74 & 0.72 & 0.71 & 0.72 & 51.46 \\
\hline & OVG3P3T4 & 28.93 & 74 & 0.71 & 0.71 & 0.71 & 51.28 \\
\hline & OVG3P4T1 & 28.93 & 74 & 0.73 & 0.74 & 0.74 & 52.17 \\
\hline & OVG3P4T2 & 28.93 & 74 & 0.74 & 0.74 & 0.74 & 52.35 \\
\hline
\end{tabular}


Table C.4: Phase III Test Matrix, Continued

\begin{tabular}{|c|c|c|c|c|c|c|c|}
\hline \multirow{2}{*}{ Date } & \multirow{2}{*}{ Test } & \multicolumn{2}{|c|}{ ATM } & \multicolumn{3}{|c|}{ Manometer Height (inH2O) } & \multirow{2}{*}{\begin{tabular}{|l|} 
Speed \\
$\mathrm{ft} / \mathrm{s}$
\end{tabular}} \\
\hline & & $\overline{P(\text { inHg) }}$ & $\mathrm{T}(\mathrm{OF})$ & Start & Stop & Average & \\
\hline \multirow{22}{*}{ 10/17/2002 } & OVG3P4T3 & 28.93 & $\overline{74}$ & 0.74 & 0.74 & 0.74 & 52.35 \\
\hline & OVG3P4T4 & 28.93 & $\overline{74}$ & 0.74 & 0.74 & 0.74 & 52.35 \\
\hline & OB1T5 & 28.93 & $\overline{74}$ & 0.71 & 0.7 & 0.71 & 51.10 \\
\hline & OB1T6 & 28.93 & 74 & 0.7 & 0.7 & 0.70 & 50.92 \\
\hline & OB1T7 & 28.93 & $\overline{74}$ & 0.7 & 0.7 & 0.70 & 50.92 \\
\hline & OB1T8 & 28.93 & 74 & 0.7 & 0.7 & 0.70 & 50.92 \\
\hline & OVG2C4T5 & 28.93 & 74 & 0.74 & 0.76 & 0.75 & 52.70 \\
\hline & OVG2C4T6 & 28.93 & 74 & 0.76 & 0.76 & 0.76 & 53.05 \\
\hline & OVG2C4T7 & 28.93 & $\overline{74}$ & 0.76 & 0.76 & 0.76 & 53.05 \\
\hline & OVG2C4T8 & 28.93 & 74 & 0.76 & 0.76 & 0.76 & 53.05 \\
\hline & OVG3C4T5 & 28.93 & $\overline{74}$ & 0.72 & 0.72 & 0.72 & 51.64 \\
\hline & OVG3C4T6 & 28.93 & $\overline{74}$ & 0.72 & 0.72 & 0.72 & 51.64 \\
\hline & OVG3C4T7 & 28.93 & 74 & 0.72 & 0.72 & 0.72 & 51.64 \\
\hline & OVG3C4T8 & 28.93 & $\overline{74}$ & 0.72 & 0.72 & 0.72 & 51.64 \\
\hline & OVG3C5T5 & 28.93 & $\overline{74}$ & 0.78 & 0.77 & 0.78 & 53.57 \\
\hline & OVG3C5T6 & 28.93 & $\overline{74}$ & 0.77 & 0.77 & 0.77 & 53.40 \\
\hline & OVG3C5T7 & 28.93 & $\overline{74}$ & 0.77 & 0.77 & 0.77 & 53.40 \\
\hline & OVG3C5T8 & 28.93 & 74 & 0.77 & 0.77 & 0.77 & 53.40 \\
\hline & OVG3M1T5 & 28.93 & $\overline{74}$ & 0.71 & 0.72 & 0.72 & 51.46 \\
\hline & OVG3M1T6 & 28.93 & $\overline{74}$ & 0.72 & 0.72 & 0.72 & 51.64 \\
\hline & OVG3M1T7 & 28.93 & $\overline{74}$ & 0.72 & 0.72 & 0.72 & 51.64 \\
\hline & OVG3M1T8 & 28.93 & 74 & 0.72 & 0.72 & 0.72 & 51.64 \\
\hline \multirow{28}{*}{ 10/18/2002 } & OVG3M1T9 & 29.11 & 72 & 0.72 & 0.76 & 0.74 & 52.35 \\
\hline & OVG3M1T10 & 29.11 & 72 & 0.76 & 0.75 & 0.76 & 52.88 \\
\hline & OVG3M1T11 & 29.11 & $\overline{72}$ & 0.75 & 0.74 & 0.75 & 52.53 \\
\hline & OVG3M1T12 & 29.11 & $\overline{72}$ & 0.74 & 0.74 & 0.74 & 52.35 \\
\hline & OVG3C5T9 & 29.11 & 72 & 0.72 & 0.72 & 0.72 & 51.64 \\
\hline & OVG3C5T10 & 29.11 & $\overline{72}$ & 0.72 & 0.72 & 0.72 & 51.64 \\
\hline & OVG3C5T11 & 29.11 & $\overline{72}$ & 0.72 & 0.72 & 0.72 & 51.64 \\
\hline & OVG3C5T12 & 29.11 & 72 & 0.72 & 0.71 & 0.72 & 51.46 \\
\hline & OVG3C4T9 & 29.11 & 72 & 0.74 & 0.77 & 0.76 & 52.88 \\
\hline & OVG3C4T10 & 29.11 & $\overline{72}$ & 0.77 & 0.77 & 0.77 & 53.40 \\
\hline & OVG3C4T11 & 29.11 & 72 & 0.77 & 0.78 & 0.78 & 53.57 \\
\hline & OVG3C4T12 & 29.11 & $\overline{72}$ & 0.78 & 0.77 & 0.78 & 53.57 \\
\hline & OVG2C4T9 & 29.11 & 72 & 0.71 & 0.7 & 0.71 & 51.10 \\
\hline & OVG2C4T10 & 29.11 & $\overline{72}$ & 0.7 & 0.7 & 0.70 & 50.92 \\
\hline & OVG2C4T11 & 29.11 & $\overline{72}$ & 0.7 & 0.7 & 0.70 & 50.92 \\
\hline & OVG2C4T12 & 29.11 & 72 & 0.7 & 0.705 & 0.70 & 51.01 \\
\hline & OVG3P1T5 & 29.11 & 72 & 0.76 & 0.75 & 0.76 & 52.88 \\
\hline & OVG3P1T6 & 29.11 & 72 & 0.75 & 0.76 & 0.76 & 52.88 \\
\hline & OVG3P1T7 & 29.11 & 72 & 0.76 & 0.75 & 0.76 & 52.88 \\
\hline & OVG3P1T8 & 29.11 & 72 & 0.75 & 0.75 & 0.75 & 52.70 \\
\hline & OVG3P2T5 & 29.11 & 72 & 0.75 & 0.76 & 0.76 & 52.88 \\
\hline & OVG3P2T6 & 29.11 & 72 & 0.76 & 0.76 & 0.76 & 53.05 \\
\hline & OVG3P2T7 & 29.11 & 72 & 0.76 & 0.76 & 0.76 & 53.05 \\
\hline & OVG3P2T8 & 29.11 & 72 & 0.76 & 0.76 & 0.76 & 53.05 \\
\hline & OVG3P3T5 & 29.11 & 72 & 0.7 & 0.7 & 0.70 & 50.92 \\
\hline & OVG3P3T6 & 29.11 & 72 & 0.7 & 0.7 & 0.70 & 50.92 \\
\hline & OVG3P3T7 & 29.11 & 72 & 0.7 & 0.69 & 0.70 & 50.73 \\
\hline & OVG3P3T8 & 29.11 & $\overline{72}$ & 0.69 & 0.69 & 0.69 & 50.55 \\
\hline
\end{tabular}


Table C.5: Phase III Test Matrix, Continued (2)

\begin{tabular}{|c|c|c|c|c|c|c|c|}
\hline \multirow{2}{*}{ Date } & \multirow{2}{*}{ Test } & \multicolumn{2}{|c|}{ ATM } & \multicolumn{3}{|c|}{ Manometer Height (inH2O) } & \multirow{2}{*}{$\begin{array}{l}\text { Speed } \\
\mathrm{ft} / \mathrm{s}\end{array}$} \\
\hline & & $\mathbf{P}$ (inHg) & $\mathrm{T}(\mathrm{oF})$ & Start & Stop & Average & \\
\hline \multirow{4}{*}{ 10/18/2002 } & OVG3P4T5 & 29.11 & 72 & 0.8 & 0.8 & 0.80 & 54.43 \\
\hline & OVG3P4T6 & 29.11 & 72 & 0.8 & 0.795 & 0.80 & 54.35 \\
\hline & OVG3P4T7 & 29.11 & 72 & 0.795 & 0.795 & 0.80 & 54.26 \\
\hline & OVG3P4T8 & 29.11 & 72 & 0.795 & 0.795 & 0.80 & 54.26 \\
\hline \multirow{36}{*}{ 10/19/2002 } & OVG3P4T9 & 28.91 & 74 & 0.71 & 0.705 & 0.71 & 51.19 \\
\hline & OVG3P4T10 & 28.91 & 74 & 0.705 & 0.7 & 0.70 & 51.01 \\
\hline & OVG3P4T11 & 28.91 & 74 & 0.7 & 0.695 & 0.70 & 50.83 \\
\hline & OVG3P4T12 & 28.91 & 74 & 0.695 & 0.7 & 0.70 & 50.83 \\
\hline & OVG3P3T9 & 28.91 & $\overline{74}$ & 0.71 & 0.71 & 0.71 & 51.28 \\
\hline & OVG3P3T10 & 28.91 & $\overline{74}$ & 0.71 & 0.715 & 0.71 & 51.37 \\
\hline & OVG3P3T11 & 28.91 & $\overline{74}$ & 0.715 & 0.715 & 0.72 & 51.46 \\
\hline & OVG3P3T12 & 28.91 & 74 & 0.715 & 0.72 & 0.72 & 51.55 \\
\hline & OVG3P2T9 & 28.91 & 74 & 0.78 & 0.785 & 0.78 & 53.83 \\
\hline & OVG3P2T10 & 28.91 & 74 & 0.785 & 0.78 & 0.78 & 53.83 \\
\hline & OVG3P2T11 & 28.91 & 74 & 0.78 & 0.78 & 0.78 & 53.75 \\
\hline & OVG3P2T12 & 28.91 & 74 & 0.78 & 0.78 & 0.78 & 53.75 \\
\hline & OVG3P1T9 & 28.91 & 74 & 0.725 & 0.73 & 0.73 & 51.91 \\
\hline & OVG3P1T10 & 28.91 & 74 & 0.73 & 0.725 & 0.73 & 51.91 \\
\hline & OVG3P1T11 & 28.91 & 74 & 0.725 & 0.73 & 0.73 & 51.91 \\
\hline & OVG3P1T12 & 28.91 & 74 & 0.73 & 0.73 & 0.73 & 52.00 \\
\hline & OB1T9 & 28.91 & 74 & 0.72 & 0.72 & 0.72 & 51.64 \\
\hline & OB1T10 & 28.91 & 74 & 0.72 & 0.72 & 0.72 & 51.64 \\
\hline & OB1T11 & 28.91 & 74 & 0.745 & 0.74 & 0.74 & 52.44 \\
\hline & OB1T12 & 28.91 & $\overline{74}$ & 0.74 & 0.735 & 0.74 & 52.26 \\
\hline & OB1T13 & 28.91 & 74 & 0.76 & 0.765 & 0.76 & 53.14 \\
\hline & OB1T14 & 28.91 & $\overline{74}$ & 0.765 & 0.76 & 0.76 & 53.14 \\
\hline & OB1T15 & 28.91 & $\overline{74}$ & 0.8 & 0.8 & 0.80 & 54.43 \\
\hline & OB1T16 & 28.91 & 74 & 0.8 & 0.8 & 0.80 & 54.43 \\
\hline & OLFP1T1 & 28.91 & $\overline{74}$ & 0.76 & 0.76 & 0.76 & 53.05 \\
\hline & OLFP1T2 & 28.91 & $\overline{74}$ & 0.76 & 0.755 & 0.76 & 52.97 \\
\hline & OLFP1T3 & 28.91 & 74 & 0.755 & 0.75 & 0.75 & 52.79 \\
\hline & OLFP1T4 & 28.91 & 74 & 0.75 & 0.75 & 0.75 & 52.70 \\
\hline & OLFP2T1 & 28.91 & 74 & 0.8 & 0.795 & 0.80 & 54.35 \\
\hline & OLFP2T2 & 28.91 & 74 & 0.795 & 0.795 & 0.80 & 54.26 \\
\hline & OLFP2T3 & 28.91 & 74 & 0.795 & 0.79 & 0.79 & 54.18 \\
\hline & OLFP2T4 & 28.91 & 74 & 0.79 & 0.79 & 0.79 & 54.09 \\
\hline & OLFP3T1 & 28.91 & 74 & 0.78 & 0.79 & 0.79 & 53.92 \\
\hline & OLFP3T2 & 28.91 & 74 & 0.79 & 0.79 & 0.79 & 54.09 \\
\hline & OLFP3T3 & 28.91 & 74 & 0.79 & 0.79 & 0.79 & 54.09 \\
\hline & OLFP3T4 & 28.91 & 74 & 0.79 & 0.79 & 0.79 & 54.09 \\
\hline
\end{tabular}


Table C.6: Phase III Test Matrix, Continued (3)

\begin{tabular}{|c|c|c|c|c|c|c|c|}
\hline \multirow{2}{*}{ Date } & \multirow{2}{*}{ Test } & \multicolumn{2}{|c|}{$\overline{\text { ATM }}$} & \multicolumn{3}{|c|}{ Manometer Height (inH2O) } & \multirow{2}{*}{\begin{tabular}{|l|} 
Speed \\
$\mathrm{ft} / \mathrm{s}$
\end{tabular}} \\
\hline & & $\mathrm{P}(\mathrm{inHg})$ & $\mathrm{T}(\mathrm{oF})$ & Start & Stop & Average & \\
\hline \multirow{19}{*}{$10 / 21 / 2002$} & TSARSIDE & 29.05 & 78 & in data file & for compari & son & \\
\hline & & \multicolumn{5}{|c|}{ Test section area reduced - side velocity determination } & \\
\hline & TSARBT1 & 29.05 & 78 & 0.74 & 0.745 & 0.74 & 52.44 \\
\hline & TSARBT2 & 29.05 & 78 & 0.745 & 0.745 & 0.75 & 52.53 \\
\hline & TSARBT3 & 29.05 & 78 & 0.745 & 0.74 & 0.74 & 52.44 \\
\hline & TSARBT4 & 29.05 & 78 & 0.74 & 0.74 & 0.74 & 52.35 \\
\hline & TSARVG1C2T1 & 29.05 & 78 & 0.73 & 0.74 & 0.74 & 52.17 \\
\hline & TSARVG1C2T2 & 29.05 & 78 & 0.74 & 0.74 & 0.74 & 52.35 \\
\hline & TSARVG1C2T3 & 29.05 & 78 & 0.74 & 0.74 & 0.74 & 52.35 \\
\hline & TSARVG1C2T4 & 29.05 & 78 & 0.74 & 0.735 & 0.74 & 52.26 \\
\hline & TSARVG3P3T1 & 29.05 & 78 & 0.8 & 0.8 & 0.80 & 54.43 \\
\hline & TSARVG3P3T2 & 29.05 & 78 & 0.8 & 0.8 & 0.80 & 54.43 \\
\hline & TSARVG3P3T3 & 29.05 & 78 & 0.8 & 0.8 & 0.80 & 54.43 \\
\hline & TSARVG3P3T4 & 29.05 & 78 & 0.8 & 0.795 & 0.80 & 54.35 \\
\hline & OSIDE & 29.05 & 78 & in data file & for compari & son & \\
\hline & $\overline{\mathrm{OB} 1 \mathrm{~T} 17}$ & 29.05 & 78 & 0.795 & 0.795 & 0.80 & 54.26 \\
\hline & OB1T18 & 29.05 & 78 & 0.795 & 0.795 & 0.80 & 54.26 \\
\hline & OB1T19 & 29.05 & 78 & 0.795 & 0.795 & 0.80 & 54.26 \\
\hline & OB1T20 & 29.05 & 78 & 0.795 & 0.79 & 0.79 & 54.18 \\
\hline
\end{tabular}

